\title{
Micromethodology for the characterization of hemoglobin variants
}

Citation for published version (APA):

Plaseska, D. (1994). Micromethodology for the characterization of hemoglobin variants. [Doctoral Thesis, Maastricht University]. Rijksuniversiteit Limburg. https://doi.org/10.26481/dis.19940428dp

Document status and date:

Published: 01/01/1994

DOI:

10.26481/dis.19940428dp

Document Version:

Publisher's PDF, also known as Version of record

\section{Please check the document version of this publication:}

- A submitted manuscript is the version of the article upon submission and before peer-review. There can be important differences between the submitted version and the official published version of record.

People interested in the research are advised to contact the author for the final version of the publication, or visit the DOI to the publisher's website.

- The final author version and the galley proof are versions of the publication after peer review.

- The final published version features the final layout of the paper including the volume, issue and page numbers.

Link to publication

\footnotetext{
General rights rights.

- You may freely distribute the URL identifying the publication in the public portal. please follow below link for the End User Agreement:

www.umlib.nl/taverne-license

Take down policy

If you believe that this document breaches copyright please contact us at:

repository@maastrichtuniversity.nl

providing details and we will investigate your claim.
}

Copyright and moral rights for the publications made accessible in the public portal are retained by the authors and/or other copyright owners and it is a condition of accessing publications that users recognise and abide by the legal requirements associated with these

- Users may download and print one copy of any publication from the public portal for the purpose of private study or research.

- You may not further distribute the material or use it for any profit-making activity or commercial gain

If the publication is distributed under the terms of Article $25 \mathrm{fa}$ of the Dutch Copyright Act, indicated by the "Taverne" license above, 


\title{
MICROMETHODOLOGY FOR THE CHARACTERIZATION
}

\author{
OF HEMOGLOBIN VARIANTS
}

\section{PROEFSCHRIFT}

ter verkrijging van de graad van doctor aan de Rijksuniversiteit Limburg te Maastricht, op gezag van de Rector Magnificus, Prof. Dr. H. Philipsen, volgens het besluit van het College van Dekanen,

in het openbaar te verdedigen

op donderdag, 28 april 1994 om 14.00 uur

door

\section{Dijana Plaseska}

geboren te Skopje op 21 mei 1963 


\section{Promotores:}

Prof. Dr. H.C. Hemker

Prof. Dr. T.H.J. Huisman, Medical College of Georgia, USA

\section{Beoordelingscommissie:}

Prof. Dr. P.J. Brombacher (voorzitter)

Prof. Dr. L.F. Bernini, Rijksuniversiteit Leiden

Prof. Dr. G.D. Efremov, Macedonian Academy of Sciences and Arts Prof. Dr. J.P.M. Geraedts

Prof. Dr. W. van der Slik, Rijksuniversiteit Groningen 


\section{ACKNOLLEDGEMENTS}

I am most grateful to Professor Dr. Titus H.J. Huisman, without whose direction and continuous support the work presented in this dissertation would not have been accomplished. His advice, encouragement, and forbearance is deeply appreciated. I would like to express my deepest thanks to Professor Dr. H.C. Hemker for his help in the realization of this dissertation. I am glad to be able to record my indebtedness to Professor Dr. Georgi D. Efremov, who has most profoundly influenced my research involvement in the field of hemoglobinopathies. The secretarial, technical, and editorial expertise of Mrs. Marianne F.H. Carver was indispensable in the preparation of this manuscript. Finally, I would like to acknowledge the participation of a number of colleagues who have either provided blood samples or have contributed in the experimental part of the work presented here. To all of them I express my deepest gratitude. 
To my parents with all my love 


\section{CONTENTS}

\section{Abbreviations}

1 Introduction and Review of the Literature

la Addundum

$2 \quad$ Materials and Methods

a Chain Variants Identified by HPLC

3a Hb Davenport or $a_{2} 78$ (EF7)Asn->His $\beta_{2}$

3b Hb Sun Prairie or $\alpha_{2} 130(\mathrm{H} 13)$ Ala->ProB $B_{2}$; Second Observation in an Indian Adult

B Chain Variants Identified by HPLC

4a $\mathrm{Hb}$ Iowa or $\alpha_{2}{ }_{2} 119\left(\mathrm{GH}_{2}\right)$ Trp->Arg

$4 b \quad \mathrm{Hb}$ Zengcheng or $\alpha_{2}^{\beta} 2^{114}$ (G16)Leu->Met

B Chain Variants Identified by HPLC and DNA Analyses

5a Hb Yokohama [B31(B13)Leu->Pro] Detected as a De Novo Mutation in a Yugoslavian Boy

5b Hb Yolga [B27(B9)Ala->Asp]: Detection of a De Novo Mutation by Ava II Digestion of PCRAmplified DNA

6a Hb F-Jiangsu, the First $Y$ Chain Variant With a Valine->Hethionine Substitution: $a_{2} A_{Y_{2} 134}$ (H12)Va1->Met

6b Hb F-Catalonia or $\alpha_{2}{ }^{C_{\gamma}}$ 15(A12)Trp->Arg

6c Hb F-Brooklyn or $\alpha_{2} G_{Y_{2}} 66(E 10)$ Lys->GIn

Hb Montrea1, a Variant With an Extended B Chain Due to a Deletion and an Insertion at the Same Location Identified by Sequencing of Amplified DNA 


\section{ABBREVIATIOHS}

$\begin{array}{ll}\text { AE } & \text { aminoethylated } \\ \text { ASO } & \text { allele specific oligonucleotide } \\ \text { bP } & \text { base pair(s) } \\ \text { BSA } & \text { bovine serum albumin } \\ \text { CCl } 4 & \text { carbontetrachloride } \\ \text { CD(s) } & \text { codon(s) } \\ \text { CF } & \text { cystic fibrosis } \\ \text { CM- } & \text { carboxymethyl- } \\ \text { CO2 } & \text { carbondioxide } \\ \text { DABITC } & \text { 4-NN-dimethylaminoazobenzene-4-isothiocyanate } \\ \text { dATP } & \text { deoxy adenosine triphosphate } \\ \text { ddATP } & \text { dideoxy adenosine triphosphate } \\ \text { ddCTP } & \text { dideoxy cytosine triphosphate } \\ \text { ddGTP } & \text { dideoxy guanosine triphospate } \\ \text { ddTTP } & \text { dideoxy thymidine triphosphate } \\ \text { DEAE- } & \text { diethylaminoethyl- } \\ \text { dGTP } & \text { deoxy guanosine triphosphate } \\ \text { DNA } & \text { deoxyribonucleic acid } \\ \text { DPG } & \text { (2,3)-diphosphoglycerate } \\ \text { DTT } & \text { dithiothreitol } \\ \text { dTTP } & \text { deoxy thymidine triphosphate } \\ \text { EDTA } & \text { ethylene diamine tetra acetatedisodium } \\ \text { FSC } & \text { frameshift } \\ \text { Hb(s) } & \text { hemoglobin(s) } \\ \text { HPFH } & \text { hereditary persistence of fetal Hb } \\ \text { HPLC } & \text { high performance liquid chromatography } \\ \text { HS } & \text { hypersensitive site(s) } \\ \text { IEF } & \text { isoelectrofocusing } \\ \text { IVS } & \text { intervening sequence } \\ \text { kb } & \text { kilobase(s) } \\ \text { LCR } & \text { locus control region } \\ \text { MCH } & \text { mean corpuscular Hb } \\ \text { MCHC } & \text { mean corpuscular Hb concentration } \\ \text { MCV } & \text { mean corpuscular volume } \\ \text { MOPS } & \text { (3[N-morpholino]propanesulfonic acid) } \\ \text { mRNA } & \text { messenger ribonucleic acid } \\ \text { O2 } & \text { oxygen } \\ \text { nt(s) } & \text { nucleotide(s) } \\ \text { PAGE } & \text { polyacrylamide gel electrophoresis } \\ \text { PCMB } & \text { p-chloromercuribenzoate } \\ \text { PCR } & \text { polymerase chain reaction } \\ \text { PCV } & \text { packed cell volume } \\ \text { PITC } & \text { phenylisothiocyanate } \\ \text { poly A } & \text { polyadenylation } \\ \text { PTC } & \text { phenylthiocarbamyl } \\ \text { PVP } & \text { polyvynilpyrolidone } \\ \text { RBC(s) } & \text { red blood cell(s) } \\ \text { RET(s) } & \text { retention time(s) } \\ \text { SDS } & \text { sodium dodecilsulphate } \\ & \end{array}$




$\begin{array}{ll}\text { SS } & \text { sickle cell anemia } \\ \text { SSC } & \text { sodium saline citrate buffer } \\ \text { SSPE } & \text { sodium saline phosphate EDTA buffer } \\ \text { TMAC } & \text { tetramethyl ammonium chloride } \\ \text { TBE } & \text { Tris borate EDTA buffer } \\ \text { TE } & \text { Tris EDTA buffer } \\ \text { TEA } & \text { Tris EDTA acetate buffer } \\ \text { TFA } & \text { trifluoroacetic acid } \\ \text { thal } & \text { thalassemia } \\ \text { Tm } & \text { melting temperature } \\ \text { TMB } & 5,5^{\prime} \text {-tetramethylbenzidine } \\ \text { UTR(s) } & \text { untranslated region(s) }\end{array}$




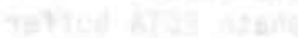

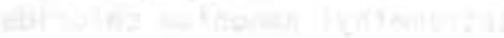

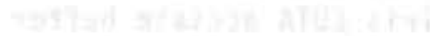

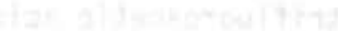

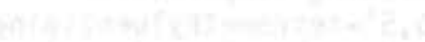

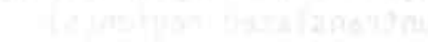


CHAPTER 1

I NT R OD U C TI ON

A N D

REVIEW OF THE LITERATURE 
and hate 


\section{CHAPTER 1}

\section{IATRODUCTION}

$\mathrm{Hb}$ has played a historic role in many scientific disciplines such as chemistry, biology, genetics, and medicine. The important functional role of $\mathrm{Hb}$ and the relative ease of isolating it in pure form have led to an early characterization, much earlier than that of any other human protein. The amino acid sequences of the globin subunits were among the first to be determined. The three-dimensional structure of $\mathrm{Hb}$ has also been determined in detail. The functional properties of the Hb molecule, the factors regulating its activity, and the structural features that underlie its functional behavior, are also well understood. The Hb molecule has served as a model for the studies of the structure, and structurefunction relationships of proteins in general.

The numerous naturally occurring mutations in the globin genes have made $\mathrm{Hb}$ a model for studies of the molecular basis of genetic diseases and of gene expression. The mutations in the various globin genes are caused by a variety of mechanisms, including substitutions of one or more nts in the DNA, nt deletions or insertions, and various forms of genetic recombinations. Some of these mutations alter the structure of the globin, thus producing different abnormal gene products or abnormal Hbs, while others decrease the rate of synthesis of the globin chains, leading to the syndromes known as thalassemias.

Since 1949, when $\mathrm{Hb} S$ was found to be the cause of sickle cell anemia, the number of $\mathrm{Hb}$ variants has increased to more than 600 . The clinical and hematological consequences have in about one-third of all cases been of sufficient severity to bring the affected individuals to medical attention; this has promoted the detection and identification of some of these abnormalities. In addition, numerous population surveys have been conducted in many areas of the world, resulting in the identification of a large number of rare and clinically silent $\mathrm{Hb}$ variants. They have also yielded valuable data about some of the more common Hb abnormalities.

Much progress has been made in the studies of the Hb variants, mainly because of technological advances that have provided more sensitive methods for their detection, separation, and characterization. Electrophoretic techniques have undoubtedly played the most important role, and these are still the most common methods used for the initial detection of abnormal Hbs. Chromatographic procedures that permit the separation and isolation of mutant $\mathrm{Hbs}$ and of individual globin chains have played an important role in the structural analyses of $\mathrm{Hb}$ variants. Still, both electrophoretic and classical chromatographic techniques fail to separate many abnormal Hbs. The development of HPLC has provided a new research tool for the detection of $H b$ variants and for the separation and isolation of abnormal globin chains and peptides. The HPLC techniques have the advantage of being fast, reproducible, and sensitive with a high resolving power. Recently, the techniques of recombinant DNA technology, widely used for delineating the molecular pathology of the thalassemia syndromes, have 
been applied to identifying structurally different Hb types. The sequence analysis of amplified DNA has been shown to be useful in the characterization of the $\mathrm{Hb}$ variants, especially of those that are present in low amounts. In some instances, the identification at DNA level is necessary to confirm and/or explain the molecular mechanism responsible for the occurrence of some variants.

The main goal of the studies reported in this dissertation is to demonstrate the usefulness of the HPLC techniques and DNA methods for the characterization of rare $\mathrm{Hb}$ variants, and to contribute to our understanding of molecular mechanisms that are responsible for the occurrence of some unusual $\mathrm{Hb}$ abnormalities.

\section{NORMAL AND ABNOPMAL HUMAN HBS: A REVIEM OF THE NORMAL HUMAN HBS}

Hb Structure and Function. The human $\mathrm{Hb}$ molecule is a tetramer with a molecular weight of about 64,000 . It is composed of two pairs of two structurally different polypeptide chains. Each of these globin chains contains a heme group that is protoporphyrin IX complexed with an iron atom. Several $\mathrm{Hb}$ types are synthesized in human red cells during the different stages of development. The Hbs Gower-I ( $5 \approx \varepsilon 2)$, Gower-II ( $\alpha 2 \varepsilon 2$ ),

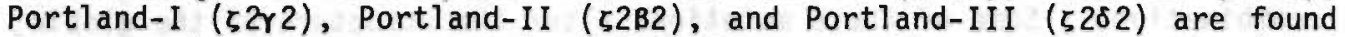
in the early embryonic period; $H b F$ is the major $H b$ component during fetal life and in newborns, while $\mathrm{Hb} A$ as major, and $\mathrm{Hb} \mathrm{A}_{2}$ as minor $\mathrm{Hb}$ component, are present in adults (1).

The amino acid sequences of all normal human globin chains have been determined (2-7). The $\alpha-1$ ike chains ( $\alpha$ and $\zeta$ ) contain 141, and the non- $\alpha$ or $\beta-1$ ike chains ( $\beta, \delta, \gamma$, and $E$ ) 146 amino acid residues. The $\zeta$ chain is similar to the a chain, differing in 57 of its 141 residues, while the $E$, $\gamma$, and $\delta$ chains resemble the $B$ chain, differing in 36,39 , and 10 positions, respectively.

Although the a and non-a chains differ considerably in their primary structure, they have similar secondary and tertiary structures (8). About $75 \%$ of the globin chain is in the shape of a right-handed a-helix (9). The a chain has eight helical segments, designated $A$ through $H$. The $B$ chain contains seven helices, closely comparable to those of the a chain, except for the $D$ helix which is absent. The a-helix is interrupted by non-helical segments that allow folding. Proline is the usual participant of the nonhelical regions since it is unable to participate in the a-helix, except as one of the first three residues from the $\mathrm{N}$-terminal end (10). The globin chains are folded in such a way that amino acids with predominantly hydrophilic polar side groups are located on the exterior surface, while amino acid residues with almost exclusively non-polar, hydrophobic groups are buried within the globin subunit away from water (10). Each chain has some invariant amino acid residues that are homologous in the comparable helices of the $\alpha$ and $\boldsymbol{\beta}$ chains; for instance, the proximal histidine at helix F8 ( $\alpha 87, \beta 92)$ and the distal histidine at helix E7 ( $\alpha 58, \beta 63)$, and tyrosine at helix $H 2(\alpha 140, B 145)$ (11). 
The heme iron is inserted in a cleft formed by the $E$ and $F$ helices of the globin chain and is covalently linked to the imidazole nitrogen of the proximal F8 histidine. In addition, a large number of relatively weak hydrophobic bonds are formed between the heme ring and about 20 side chains of the globin, that stabilize the heme group and the hydrophobic interior of the heme pocket (12). Thus, the heme iron is situated in a non-polar, hydrophobic environment, that facilitates the iron's ability to combine reversibly with molecular $\mathrm{O}_{2}$ without being oxidized.

The quaternary structure of the $\mathrm{Hb}$ molecule was determined by Perutz (13); it changes markedly in going from the oxy ( $R$ or relaxed) to the deoxy ( $T$ or tense) form. The subunits of $\mathrm{Hb}$ interact with each other by means of relatively weak, noncovalent, bonds such as van der Waals forces and hydrogen bonds. There are two types of contacts between unlike subunits that are called the $\alpha 1 B 1 \quad(\alpha 2 B 2$ ) and $\alpha 1 B 2$ ( $\alpha 2 B 1$ ) contacts (14). The $\alpha 1 B 1$ interface involves 40 contacts between residues, including nine hydrogen bonds. Since the al $B 1$ interface is extensive and rather rigid, and remains relatively fixed during oxygenation, the contacts between the $\alpha 1$ and $B 1$ subunits are identical in oxyHb and deoxyHb. The alB2 interface however, undergoes a considerable shift in position during oxygenation. The primary sites for contact are the FG corner (i.e. the non-helical segment between the $F$ and $G$ helices) and the $C$ helix of one subunit with complementary ( $C$ and $F G$ ) regions of the other subunit. Many of the a1B2 bonds that are present in the oxy molecular state are broken with the shift to the deoxy conformation, and a new set of bonds is formed. In deoxyHb there are about 40 contacts, including 19 hydrogen bonds. Upon oxygenation, the total number of contacts increases to 22, including 12 hydrogen bonds. The dissociation of the oxyHb under physiological conditions into identical dimers occurs at the $\alpha 182$ interface, while monomer formation takes place at the al $\mathrm{Bl}$ interface, but only at $\mathrm{pH}$ values below 5 and above 11 (15).

DeoxyHb is stabilized by a number of inter- and intrasubunit salt bonds that are absent or very much weaker in oxyHb. The C-terminal residues of both $\alpha$ and $\beta$ chains ( $\alpha 141 \mathrm{Arg}$ and $B 146 \mathrm{His}$ ) are particularly involved in intersubunit salt bridges (16). In contrast to the numerous contacts between unlike chains, contacts between similar chains ( $\alpha$ la2 and B1 B2) are minimal (16). Like chains are separated by an internal cavity line by polar amino acids. The two $B$ chains are widely separated in the deoxy conformation, which makes it possible to accommodate a molecule of the allosteric effecter 2,3-DPG (17). In the oxy conformation, the gap is closed when the $B$ chains are moving together. The channel between the two chains was found to form a receptor site for certain drugs (18).

The primary functional role of $\mathrm{Hb}$ is the transport of $\mathrm{O}_{2}$ from the lungs to the body tissues and that of $\mathrm{CO}_{2}$ in the reverse direction. The $\mathrm{O}_{2}$ binds reversibly to the heme iron of each of the four $\mathrm{Hb}$ subunits. As each $\mathrm{O}_{2}$ molecule binds to the $\mathrm{Hb}$ molecule, the affinity of $\mathrm{Hb}$ for the next $\mathrm{O}_{2}$ molecule increases. The important consequence of this cooperation (heme-heme interaction) is that a small decrease in the $\mathrm{O}_{2}$ tension, such as that between the lung and tissue capillaries, results in the release of a sufficient amount of $\mathrm{O}_{2}$ from the blood (15). 
The cooperativity of $\mathrm{Hb}$, proposed by Perutz, arises from an equilibrium between the oxy and deoxy quaternary structure (13). In the oxy conformation, the iron atoms of the heme groups lie nearly within the plane of the heme group. After $\mathrm{O}_{2}$ is removed, the heme iron electrons are redistributed, and the iron atom is displaced from the plane of the heme ring. This shift in the position of the iron atom is transmitted through its bond to the histidine residue at position F8, thus initiating a succession of changes in the quaternary structure of the $\mathrm{Hb}$ molecule. The direct mechanical system of transmission from the iron atoms to the salt bridges, proposed by Perutz (13), was shown to be incorrect with the Shaanan's X-ray analysis of the structure of human oxyHb at $2.1 \AA$ resolution (19). Molecules are more likely to jump across the energy barriers between two alternative structures ( $T$ and $R$ ), possibly by several alternative pathways (20).

The efficiency of the $\mathrm{O}_{2}$ transport can be determined by measuring the $\mathrm{O}_{2}$ equilibrium curve of $\mathrm{Hb}$ and $i$ ts $\mathrm{P}_{50}$ value. The $\mathrm{O}_{2}$ equilibrium curve of blood or $\mathrm{Hb}$ solution is characteristically sigmoid-shaped. The P50 represents the $\mathrm{O}_{2}$ tension at which the $\mathrm{Hb}$ molecule is half-saturated with $0_{2}$. The $P_{50}$ for human blood at physiological pH $(7.4)$, temperature $\left(37^{\circ} \mathrm{C}\right)$, 2,3-DPG concentration $(5 \mathrm{mM})$, and $\mathrm{pCO}_{2}(5.3 \mathrm{kPa})$ is normally $26 \pm 1 \mathrm{~mm} \mathrm{Hg}$. The $\mathrm{P}_{50}$ is inversely related to the $\mathrm{O}_{2}$ affinity. Several factors can affect the $\mathrm{O}_{2}$ affinity of $\mathrm{Hb}$ such as: Temperature, $\mathrm{pH}, \mathrm{CO}_{2}, 2,3-\mathrm{DPG}$ binding, and chloride ions (15).

The $\mathrm{O}_{2}$ affinity decreases with an increase in temperature. The $\mathrm{P}_{50}$ of normal human blood is almost doubled by an increase in temperature from $20^{\circ} \mathrm{C}$ to $30^{\circ} \mathrm{C}(21)$. This effect is physiologically important; during fever, metabolism is increased dramatically and an increase in the delivery of $\mathrm{O}_{2}$ is required. Conversely, cold induces a decrease in metabolic requirements, and a reduced need for $\mathrm{O}_{2}(22)$.

The $\mathrm{O}_{2}$ affinity of $\mathrm{Hb}$ is reduced by increasing amounts of $\mathrm{CO}_{2}$, primarily due to a reduction in $\mathrm{pH}$ (23). Under physiological conditions the $\mathrm{P}_{50}$ of $\mathrm{Hb}$ varies inversely with the $\mathrm{pH}$ (alkaline Bohr effect). The alkaline Bohr effect is physiologically important; the $\mathrm{O}_{2}$ affinity of $\mathrm{Hb}$ in the tissues, where the $\left(\mathrm{H}^{+}\right)$concentration is increased, will be decreased, thus promoting the unloading of $\mathrm{O}_{2}$ from the tissues. Similarly, when $\mathrm{CO}_{2}$ is expelled through the lungs, there is a corresponding increase in $\mathrm{pH}$ and a relative increase in $\mathrm{Hb} \mathrm{O}_{2}$ affinity, that will favor the uptake of $\mathrm{O}_{2}$. Residues B146 His and al Val contribute significantly to the alkaline Bohr effect $(24,25)$. Other residues, such as $B 82$ Lys may also be important participants in the Bohr effect (24).

In addition to the $\mathrm{CO}_{2}$ transport (as bicarbonate ions and protons) a portion of $\mathrm{CO}_{2}$ is bound chemically to $\mathrm{Hb}$ through formation of carbamates with the $N$-termini of $\alpha$ and $B$ chains (26). Under physiologic conditions, only about $10 \%$ of $\mathrm{CO}_{2}$ produced by respiring tissues, is transported as a carbamino complex with $\mathrm{Hb}(27)$.

The $\mathrm{O}_{2}$ affinity of $\mathrm{Hb}$ is strongly influenced by 2,3-DPG, the most abundant organic phosphate in human erythrocytes $(28,29)$. An increase in the concentration of 2,3-DPG will increase the $\mathrm{O}_{2}$ affinity of $\mathrm{Hb}$. Under 
physiologic $\mathrm{pH}$ and ionic strength the 2,3-DPG binds more strongly to deoxy$\mathrm{Hb}$. One molecule of 2,3-DPG binds to one molecule of deoxyHb (30). The amino acid residues involved in the binding of 2,3-DPG are: Val 1, His 2, Lys 82 , and His 143 of each $B$ chain (31). Position 143 of the $\gamma$ chain is occupied by a serine residue instead of histidine, which decreases the binding of 2,3-DPG to Hb $F(32)$. Although the levels of 2,3-DPG are similar in fetal and adult red cells, this decreased binding of 2,3-DPG to $\mathrm{Hb} F$ results in a higher $\mathrm{O}_{2}$ affinity of fetal blood when compared to adult blood (33).

Chloride ions decrease the $\mathrm{O}_{2}$ affinity of the $\mathrm{Hb}$ through binding to the N-terminal amino group of the a chain and $B 82$ Lys (34). Because the latter is an important 2,3-DPG binding site, it probably does not bind significant amounts of chloride under physiologic conditions.

Genomic Organization of Human Globin Genes. The synthesis of the human globin chains is regulated by two gene clusters: The $\alpha$-globin gene cluster is located on the short arm of chromosome 16 and that of the $\beta$ globin gene on the short arm of chromosome $11(35-41)($ Fig. 1/1). The $\alpha-g l o b i n g e n e$ cluster consists of duplicated a genes ( $\alpha 1$ and $\alpha 2$ ), one embryonic gene $(\zeta)$, three $\psi$ genes $(\psi \alpha 1, \psi \alpha 2$, and $\psi \zeta)$, and one gene with an, as yet, undetermined function (the $\theta 1$ gene), arranged in the following order: $5^{\prime}-\zeta-\psi \zeta-\psi \alpha 2-\psi \alpha 1-\alpha 2-\alpha 1-\theta 1-3^{\prime}$. The $B-g$ lobin gene cluster includes six genes: One embryonic $(\varepsilon)$ gene, duplicated $Y\left(G_{\gamma}\right.$ and $\left.A_{\gamma}\right)$ genes, adult $\beta$ and $\delta$ genes, and one $\psi$ gene $(\psi \beta)$ in this order: $5^{\prime}-E-G_{\gamma-} A_{\gamma-\psi \beta-\delta}-\beta-3^{\prime}$. It is likely that the genes found in both globin gene clusters have arisen by gene duplication events, followed by mutation and subsequent inactivation. An intriguing feature of the linear organization of the globin genes on both clusters is that they are arranged on the chromosome in the same order in which they are expressed during development. An additional interesting feature of the chromosomal arrangement is that the globin gene pairs which are expressed together during development (the two $\alpha$ genes, the $\delta$ and $\beta$ genes, the $G_{\gamma}$ and $A_{\gamma}$ genes) are spaced relatively close together, whereas larger distances separate the genes that are expressed at different developmental periods.

All globin genes have a similar structure consisting of three coding regions (exons 1,2 , and 3 ), two IVS (introns 1 and 2), a $5^{\prime}$ promoter region, and a $3^{i}$ UTR $(32-47)$ (Fig. 1/2). The promoter region, located 100-200 bp upstream from the start site of transcription (Cap site), contains sequences necessary for an accurate and efficient initiation of transcription (48). The most proximal is the so-called TATA box, located at $-30 \mathrm{bp}$. The CCAAT box is located at around $-70 \mathrm{bp}$, and is duplicated in the $r$-globin gene promoter. The third sequence motif, called CACCC, is duplicated in the $\beta$-globin gene and is located at -90 and $-105 \mathrm{bp}$. In the $\delta$-globin gene promoter, the CCAAT and CACCC boxes have modified structures, which may account for its low level of expression.

The coding region of the globin genes is contained in three exons. To some extent it is possible to correlate the distinct functions of $\mathrm{Hb}$ with each of the exons $(50,51)$. Exon 2 encodes for the segment involved in heme binding and with alB2 contact formation. Amino acid residues that 
participate in the al $\mathbf{B l}$ contact formation are mainly derived from the third exon, whereas those that contribute to the Bohr effect and to 2,3-DPG formation are more randomly distributed.

Chromosome "16

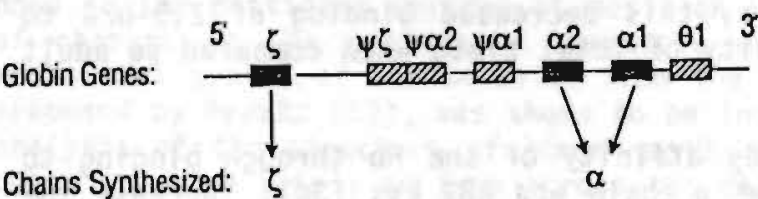

Hb Types:
Chromosome \#11
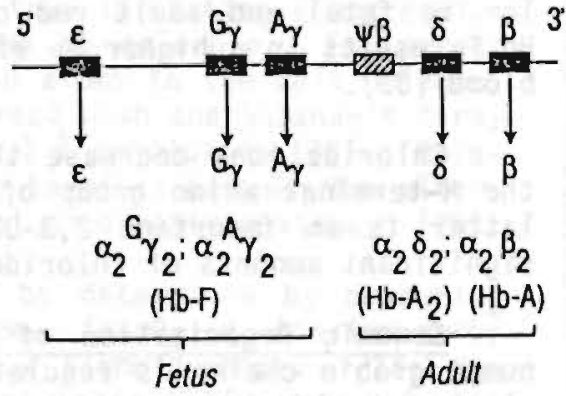

FIG. 1/1. Organization of the globin genes on chromosomes 11 and 16 . The black boxes indicate functional genes. The seven normal $\mathrm{Hb}$ types, synthesized at particular developmental stages are also presented (from Ref. 1).

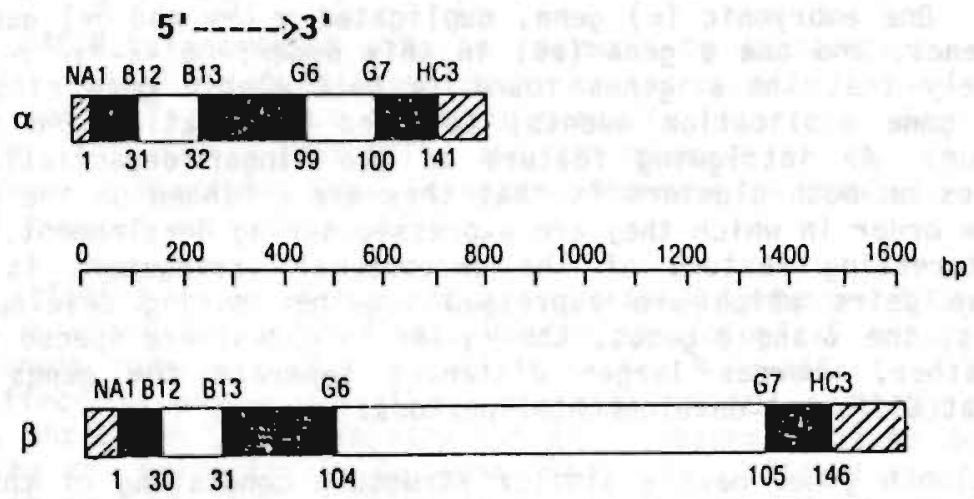

FI6. 1/2. Fine structure of human $\alpha$ - and B-globin genes. The black blocks represent the coding regions, the white blocks indicate the IVS, and the hatched blocks at either end represent the $5^{\prime}$ and $3^{\prime}$ UTR. The numbers below the diagrams correspond to the amino acid CD positions; the helical positions are given above.

In the 8 -like globin genes the IVS interrupt the coding sequence between CDs 30 and 31 , and between CDs 104 and 105, while in the a-globin gene family they interrupt the coding region between CDs 31 and 32 , and CDs 99 and 100. Although the precise CD position numbers differ between the $\alpha$ - and $\beta-1$ ike globin genes, with regard to their helical positions, the interruptions occur precisely at the same positions (Fig. 1/2). The IVS are included in the precursor mRNA transcript in the nucleus, but are removed prior to expulsion of the mRNA to the cytoplasm by a process known as splicing (52). Conserved sequences, mainly the invariant GT and AG 
dinucleotide sequences at the $5^{\prime}$ and $3^{\prime}$ ends of the intron, are essential for the proper splicing of precursor mRNA molecules (53). At the $3^{\prime}$ end of the globin genes there is a highly conserved sequence AATAM that is necessary for proper processing, polyadenylation, and cleavage of mRNA transcripts (52).

Developmental Expression of the Globin Genes. During development a series of changes occur, both at the major sites of erythropoiesis and in the types of $\mathrm{Hb}$ that are synthesized (54-56). The early embryonic period is characterized by erythropoiesis in the yolk sac and the synthesis of the embryonic Hb types, e.g. Hbs Gower-I, Gower-II, Portland-I, PortlandII, and Portland-III. From the 5 th to the 13 th weeks after conception, the erythropoiesis is shifted to the fetal liver, with a consequent switch to the synthesis of $\mathrm{Hb} \mathrm{F}$. During the second trimester, a switch to the final place of erythropoiesis (the bone marrow) occurs. This period is characterized with a progressive fall in $r$-globin chain synthesis and a progressive rise in $\beta-$ and $\delta-g l o b i n$ chain synthesis. At the same time, expression of the $G_{\gamma}$ - and $A_{\gamma}$-globin genes switches from a 7 to 3 ratio observed in fetuses and newborns to a 2 to 3 ratio in most adults. The developmental expression of B-like genes is shown in Fig. 1/3.

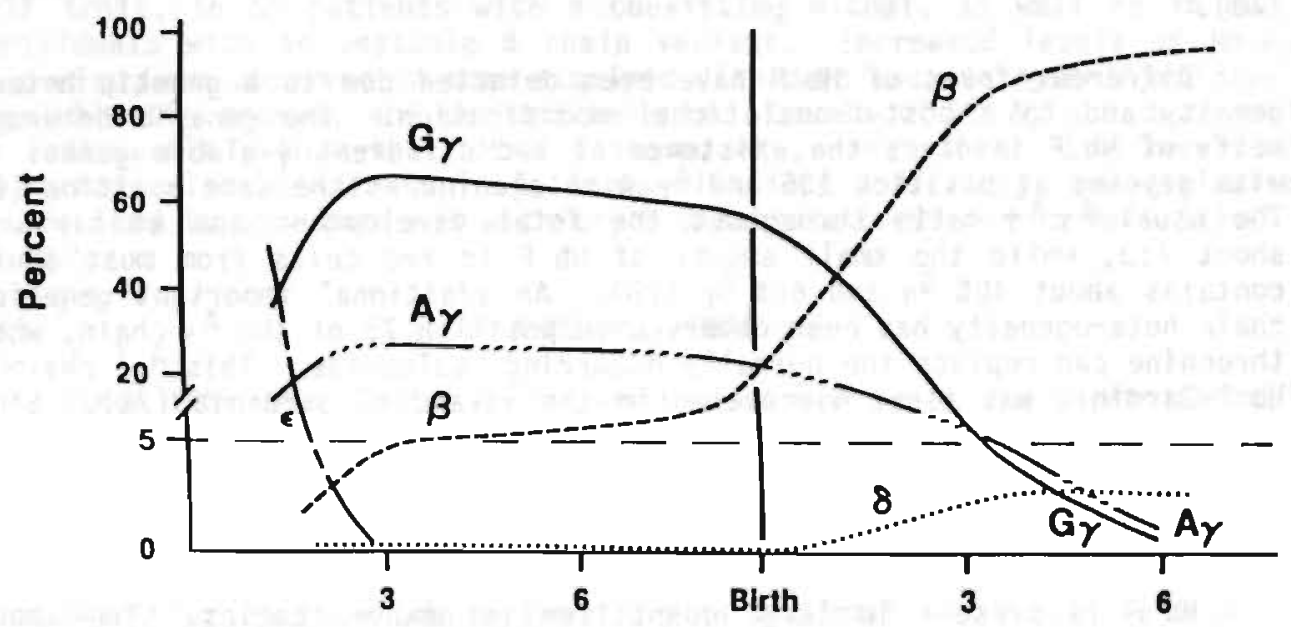

Time (months)

FIG. 1/3. Developmental expression of B-like globin genes (from Ref. 1).

Regulation of expression of the five functional globin genes is a complex mechanism and is controlled by proximal (promoters, enhancers, and silencers) and distal (LCR) cis-acting DNA sequences and trans-acting factors that bind to the regulatory DNA sequences (57). The LCR is located 6 to $20 \mathrm{~kb}$ upstream of the $\varepsilon$-globin gene and consists of four DNase I HS. Competitive interaction of the globin genes through trans-acting factors with the LCR at different stages of development is a possible mechanism through which the switching in the synthesis of the various globin chains is controlled (58). 
The Embryonic Hbs. Several embryonic Hbs are present in the early gestation period. The two embryonic Hbs, Gower-I $(52 \varepsilon 2)$ as major and Gower-II $(\alpha 2 \times 2)$ as minor component, were detected as slow-moving fractions on starch gel electrophoresis (59-61). Hb Portland-I ( $\left.{ }^{2} \gamma^{2}\right)$ was separated from $\mathrm{Hb} \mathrm{A}$ by electrophoresis at lower $\mathrm{pH}(62)$, while the minor Hbs Portland-II ( $\zeta 2 \beta 2)$ and Portland-III ( $\zeta 2 \delta 2)$ were discovered at a later time (63). The $\varepsilon$ chain has been observed only in very young fetuses. It is absent in blood samples from normal babies and from newborns with $\beta$-thal major, and in young fetuses of $16-20$ weeks (64). In contrast, the $\zeta$ chain is detectable in blood samples of about half of all normal newborns tested, and increased levels have been found in babies with various degrees of a chain deficiencies (65-67). Recent analyses of blood samples from adults with various forms of $\alpha$-thal by both immunology and HPLC methodology have detected minute quantities of $\zeta$ chain in subjects with the more severe types of deficiencies (68).

The Fetal Hbs. Hb $F$ was discovered in the middle of the previous century when the blood from a newborn baby was shown to be more resistant to alkali than that of an adult (69). Hb $F$ is formed by two $\alpha$ and two $Y$ chains, and is the predominant $\mathrm{Hb}$ during fetal life and in the neonatal period. In normal adults $\mathrm{Hb} \mathrm{F}$ accounts for less than $1 \%$ of the total $\mathrm{Hb}$ (70).

Different forms of $\mathrm{Hb} F$ have been detected due to a genetic heterogeneity and to a post-translational modification. The genetic heterogeneity of $\mathrm{HB} \mathrm{F}$ involves the existence of two different $\gamma$-globin genes: $G_{\gamma}$ with glycine at position 136 and $A_{\gamma}$ with alanine at the same position (6). The usual $\mathrm{G}_{Y} \mathrm{~A}_{Y}$ ratio throughout the fetal development and at birth is about $7: 3$, while the small amount of $\mathrm{Hb} F$ in red cells from most adults contains about $40 \% \mathrm{G}_{\gamma}$ and $60 \% \mathrm{~A}_{\gamma}(70)$. An additional important genetic $\gamma$ chain heterogeneity has been observed in position 75 of the $A_{\gamma}$ chain, where threonine can replace the normally occurring isoleucine. This $A_{\gamma}{ }^{\top}$ chain or Hb F-Sardinia was first discovered on the island of Sardinia (71). Since then it has been found in nearly all populations $(72,73)$. Furthermore, some $10-15 \%$ of $\mathrm{Hb} \mathrm{F}$ in the blood has been modified through an acetylation of the amino terminal group by an enzyme-mediated, post-synthetic process (74).

Hb $F$ is present in large quantities in newborn babies. The amount of $\mathrm{Hb} F$ decreases continuously during the first six months of life, because of a decrease in the synthesis of the $\gamma$ chains. The rate of decline of $\mathrm{Hb} F$ is somewhat slower in prematurely born babies, as well as in babies with SS and some other hemoglobinopathies (75). Simultaneously with the decrease in $\mathrm{Hb} F$, the ratio between the $G_{\gamma}$ and $A_{\gamma}$ chains changes and reaches the adult level in about three or four months. Some 20 to $30 \%$ of all babies retain their newborn ${ }^{G_{\gamma}} A_{\gamma}$ ratio of $7: 3$ as a result of a mutation in the $G_{Y}$ promoter ( $C->T$ at position -158 or $G->A$ at position $-161)(76,77)$.

Hb F levels are elevated in some normal healthy adults with a condition of HPFH. Increased levels of $\mathrm{Hb} F$ are also present in patients with SS and with a $\beta$ - or $\delta \beta$-thal homozygosity. Elevated $\mathrm{Hb} F$ levels have also 
been observed in a variety of acquired disorders, especially those with impaired erythropoiesis (reviewed in Ref. 15). A detailed review of these conditions falls outside the scope of this dissertation.

The Adult Hbs. The major $\mathrm{Hb}$ component in normal adults is $\mathrm{Hb} \mathrm{A}$. It normally makes up to $97 \%$ of the total $\mathrm{Hb}$ and is therefore the primary determinant of $\mathrm{Hb}$-related functional properties of the blood. $\mathrm{Hb} A$ is formed of two a and two B chains. Approximately $5 \%$ of $\mathrm{Hb} A$ in normal adults is present as a glycosylated derivative known as $H b A_{1}$, in which the amino terminal valyl residue of the $B$ chain is in ketoamine linkage with glucose (78). $\quad \mathrm{Hb} \mathrm{A}_{1}$ is the product of a non-enzymatic, post-synthetic, glycosylation of $H b A$, and is elevated 2- to 3-fold in individuals with an untreated diabetes mellitus who have persistent hyperglycemia (79).

$\mathrm{Hb} \mathrm{A}_{2}$ represents a minor $\mathrm{Hb}$ fraction, accounting for $2-3 \%$ of the $\mathrm{Hb}$ in erythrocytes of normal adults (80). Hb $A_{2}$ is formed by two a and two $\delta$ chains. The functional properties of $\mathrm{Hb} \mathrm{A}_{2}$ are nearly identical to those of $\mathrm{Hb} A$, but because of its low concentration in the blood, it plays no significant role in $\mathrm{O}_{2}$ transport. Increased levels of $\mathrm{Hb} \mathrm{A}_{2}$ are present in adults with a heterozygosity for one of numerous $B$-thal mutations. Higher levels of $\mathrm{Hb} \mathrm{A}_{2}$ have also been found in individuals with sickle cell trait, in SS patients with a coexisting a-thal, as well as in many individuals with an unstable $B$ chain variant. Increased levels of $\mathrm{Hb}_{2}$ have also been observed in some acquired disorders such as hyperthyroidism, megaloblastic anemia, and perhaps malaria. Levels of $\mathrm{Hb} \mathrm{A}_{2}$ are decreased in individuals with $\alpha$-thal, $H b H$ disease, $\delta \beta-$, and $\delta$-thal. Low levels of $\mathrm{Hb} \mathrm{A}_{2}$ have also been found in patients with iron deficiency, sideroblastic anemia, and in some patients with erythroleukemia (reviewed in Ref. 15).

\section{THE ABNORAAL HUMAN HBS}

Since 1959 when sickle cell anemia was found to be due to an abnormal $\mathrm{Hb}$ with a single amino acid substitution (Glu->Val) at position 6 of the B chain (81), numerous Hb variants have been detected; at present more than $600 \mathrm{Hb}$ variants have been found (82). An updated alphabetical list of known $\mathrm{Hb}$ variants is provided as an addendum to this chapter.

Molecular Basis of $\mathbf{H b}$ Variants. Most abnormal Hbs result from mutations in the coding regions of the globin genes that change the sequences or the number of nts. Thus, most of the abnormal Hbs are characterized with single amino acid substitutions, two amino acid substitutions within the same globin chain, or with longer or shorter globin chains. Others are fusion or hybrid hbs that have arisen through crossovers between misaligned chromosomes. Table $1 / 1$ presents the total number of $\mathrm{Hbs}$ with different abnormalities reported in the literature.

Hbs With Single Amino Acid Substitutions. The great majority (around 90\%) of $\mathrm{Hb}$ variants are caused by a single amino acid replacement in the globin chain, because of a single base substitution in the globin gene. At present $559 \mathrm{Hb}$ variants are known to be the result of single point mutations (174 B, $305 \alpha, 56 \gamma$, and $24 \delta$ chain variants). Abnormal $\varepsilon$ or $\zeta$ chain 
TABLE 1/1. Total Number of Variants Reported in the Literature (Apri1 1993)

\begin{tabular}{l|rrrrr|r}
\hline & \multicolumn{5}{|c|}{ Numbers Observed } & \\
\cline { 2 - 7 } Type of Abnormality & $\boldsymbol{\alpha}$ & $\boldsymbol{B}$ & $\mathbf{G}_{\boldsymbol{\gamma}} \mathbf{a}$ & $\mathrm{A}_{\boldsymbol{\gamma}}$ & $\boldsymbol{\delta}$ & Total \\
\hline Single amino acid substitutions & 174 & 305 & 36 & 20 & 24 & 559 \\
Two amino acid substitutions & 1 & 14 & & 6 & & 21 \\
Elongated chains & 9 & 8 & & & & 17 \\
Shortened chains & 2 & 16 & & & & 18 \\
Hybrid Hbs & & & & & & 9 \\
\hline TOTAL & & & & & & 624 \\
\hline
\end{tabular}

a Includes three variants that have not been completely identified.

variants with single amino acid replacements have not been discovered. The uneven distribution of the $\mathrm{Hb}$ variants between the different globin chains can be explained by several unrelated factors. A functionally abnormal B chain variant constitutes about half of the total Hb and will contribute significantly to the function of the red cells, which at the same time, will facilitate its identification. An abnormal a chain variant is usually present at a considerably lower quantity because of the presence of three normal globin genes in a heterozygote; this is less likely to cause a significant impairment of red cell function. The relatively small number of $\delta$ chain variants that have been identified is explainable by the small amount of $\mathrm{Hb} \mathrm{A}_{2}$ that is present in the red cells. Even a $\delta$ chain variant with an altered electrophoretical mobility can easily escape detection, and those with an altered function will also likely go unrecognized. The number of $r$ chain variants that has been described is also rather small. With the newborn screening program and introduction of highly sensitive HPLC methods, new $r$ chain variants are presently being identified in a larger number than ever before.

Over 90\% of all single nt replacements occur at the first or second position of each $C D$; the second position is more frequently mutated in the $B$ - and $\delta$-globin genes, and the first position in the $\gamma$-globin genes. Variation of the nts in the third position of many CDs do not result in an amino acid change (1).

Among known $\mathrm{Hb}$ variants that have a single amino acid substitution, all except three can be explained by a single base change in the affected CD. The exceptions are: Hb Bristol [B67(E11) Val->Asp], Hb Edmonton [B50

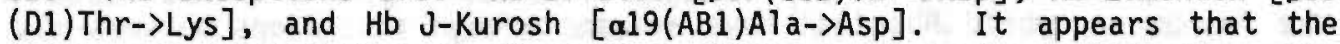
amino acid substitution in these $H b$ variants needs to be reevaluated. The possibilities that could account for such a change are either a polymorphic site at the nt position involved or two separate point mutations in the single CD (15).

Hbs Nith Two Amino Acid Substitutions. As many as 21 known Hb variants are characterized by the presence of two different amino acid substitutions within the same globin chain (Table 1/2). In most of these variants the amino acid replacement are located at widely separated sites in the globin chain. An additional interesting observation is that many of 
their individual point mutations have also been found in single substitution globin variants. For example, the amino acid replacements in the $B$ chain variant $\mathrm{Hb}$ C-Harlem (83) include the amino acid substitution of $\mathrm{Hb} \mathrm{S}$ at $\beta 6$ and of $\mathrm{Hb}$ Korlu Bu at $\beta 73$, both of them present in high frequencies in populations of West Africa (84). Six of the variants with two point mutations involve the Hb $S$ substitution. All six $Y$ chain variants with two amino acid substitutions involve the common $A_{\gamma} T^{\gamma}$ or $\left.A_{\gamma} 75(E 19) I\right] e->T h r$ substitution. These considerations have suggested that the Hbs with double point mutations arose by one of two mechanisms: A new mutation on a gene already carrying one mutation, or a crossover between two variant genes.

TABLE 1/2. Hbs With Two Amino Acid Substitutions

\begin{tabular}{|c|c|c|}
\hline Hb Name & Common Substitution & Rare or Less Cormon Substitution \\
\hline $\mathrm{J}$-Singapore & & $\begin{array}{l}\text { a 78(EF7)Asn->Asp } \\
\text { a 79(EF8)A1a->Gly }\end{array}$ \\
\hline $\begin{array}{l}\frac{\text { S-Antilles }}{\text { C-Ziguinchor }} \\
\text { C-Harlem; C-Gec } \\
\text { S-Providence } \\
\text { S-Oman } \\
\text { S-Travis }\end{array}$ & $\begin{array}{l}\beta 6(\mathrm{~A} 3) \mathrm{Gl} U->\mathrm{Val} \\
\text { same } \\
\text { eorgetown } \\
\text { same } \\
\text { same } \\
\text { same } \\
\text { same }\end{array}$ & $\begin{array}{l}\text { в 23(B5) Val->Ile } \\
\text { B 58(E2) Pro->Arg Dhofar } \\
\text { B 73(E17) Asp->Asn Korle-Bu } \\
\text { B 82(EF6) Lys->Asn->Asp Providence } \\
\text { B121(GH4) Glu->Lys 0-Arab } \\
\text { B142(H20)Ala->Val }\end{array}$ \\
\hline$\underline{\text { Arl ington } \mathrm{Pk}}$ & B 6(A3)Glu->Lys (Hb C) & B 95(FG2)Lys->Glu N-Baltimore \\
\hline T-Cambodia & в 26(B8)Glu->Lys (Hb E) & $\mathrm{B121}(\mathrm{GH} 4) \mathrm{G} 7 \mathrm{U}->\mathrm{G} 1 \mathrm{n} \underline{\mathrm{Hb} D}$ \\
\hline Grenoble & & $\begin{array}{l}\text { B } 51 \text { (D2) Pro->Ser } \\
\text { B } 52 \text { (D3) Asp->Asn 0su Christiansborg }\end{array}$ \\
\hline Poissy & & $\begin{array}{l}\text { B 56(D7) G1y->Arg Hamadan } \\
\text { B 86(F2) A1a->Pro }\end{array}$ \\
\hline Atlanta-Coventr & $\operatorname{try}$ & $\begin{array}{l}\text { B 75(E19)Leu->Pro Atlanta } \\
\text { B141(H19)Leu }>0 \text { Coventry }\end{array}$ \\
\hline$\underline{\text { Duino }}$ & & $\begin{array}{l}\text { B 92(F8) His->Pro Newcastle } \\
\text { B104(G6) Arg->Ser } \underline{\text { Camperdown }}\end{array}$ \\
\hline Masuda & & $\begin{array}{l}\text { Bl 14(G16)Leu->Met } \\
\text { Bl 19(GH2)G1y->Asp Fannin-Lubbock }\end{array}$ \\
\hline Cleveland & Bl21(GH4)Glu->Gln (Hb D) & B 93(F9) Cys->Arg Okazaki \\
\hline $\begin{array}{l}\text { F-Xinjiang } \\
\text { F-Fukuyama } \\
\text { F-Forest Park } \\
\text { F-Yamaguchi } \\
\text { F-Siena } \\
\text { F-Charlotte }\end{array}$ & $\begin{array}{c}A_{\gamma}^{\top} \text { or A } 75(E 19) \text { Ile->Thr } \\
\text { same } \\
\text { same } \\
\text { same } \\
\text { same } \\
\text { same }\end{array}$ & 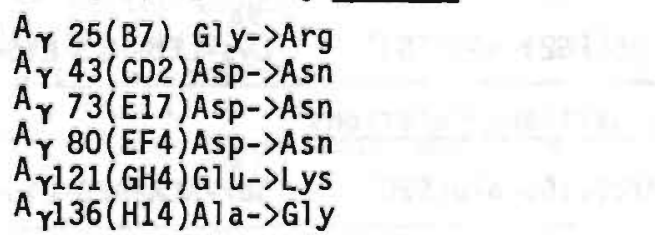 \\
\hline
\end{tabular}

Hbs With Elongated Polypeptide Chains. Thus far, $17 \mathrm{Hb}$ variants with elongated polypeptide chains have been described (Table 1/3). It is likely that they have arisen by one of the following mechanisms: Single point mutation in the chain termination CD, frameshift mutation, preservation of initiation methionine, or insertion of three or multiple of three bases. 
TABLE 1/3. Hbs With Elongated Polypeptide Chains

\begin{tabular}{|c|c|c|}
\hline Residues & Amino Acid Sequence Changes & Hb Name \\
\hline \multicolumn{3}{|l|}{$\mathrm{COOH}$ Elongations } \\
\hline $\begin{array}{l}\alpha 141(H 19) \\
\alpha 141(H 19) \\
\alpha 141(H 19) \\
\alpha 141(H 19)\end{array}$ & 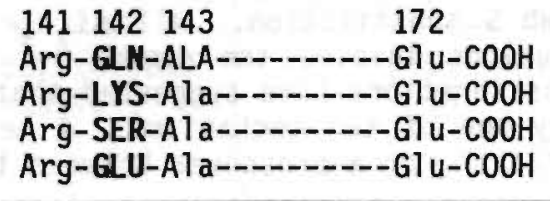 & $\begin{array}{l}\text { Constant Spring } \\
\text { Icaria } \\
\text { Koya Dora } \\
\text { Seal Rock }\end{array}$ \\
\hline \multicolumn{3}{|l|}{ Frameshifts } \\
\hline$\alpha 139(H C 1)$ & 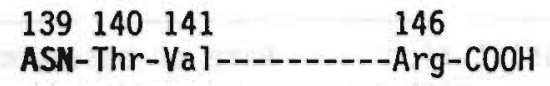 & Wayne \\
\hline$B 143(H 21)$ & $\begin{array}{ll}143144145 & 156 \\
\text { PRO-Ser-Ile-- } & \end{array}$ & Saverne \\
\hline B145(HC2) & $\begin{array}{ll}145146147 & 157 \\
\text { SER-I le-Thr- } & \end{array}$ & Cranston \\
\hline B146(HC3) & $\begin{array}{ll}146147 \quad 148 & 157 \\
\text { His-THR-Lys- }-150 \mathrm{C}\end{array}$ & Tak \\
\hline \multicolumn{3}{|l|}{$\mathrm{NH}_{2}$ Extensions } \\
\hline$\alpha 1(N A 1)$ & $\stackrel{-1+1+2}{+1}+2$ & $\underline{\text { Thionville }}$ \\
\hline $\mathrm{Bl}(\mathrm{NAl})$ & $\begin{array}{l}-1+1+2 \\
\text { MET-Val-PRO-Leu- } \\
-1+1+2\end{array}$ & Long Island-Marseille \\
\hline$B 1$ (NA1) & $\begin{array}{l}\text { MET-GLU-His-Leu- } \\
-1+1+2\end{array}$ & $\underline{\text { Doha }}$ \\
\hline B1(NA1) & MET-MET-His-Leu- & $\underline{\text { South Florida }}$ \\
\hline
\end{tabular}

\begin{tabular}{|c|c|c|}
\hline$\alpha 37(C 2)-\alpha 38(C 3)$ & $\begin{array}{l}37 \\
\text { Pro-GLU-Thr }\end{array}$ & Catonsville \\
\hline$\alpha 116(\mathrm{GH} 4)-\alpha 117(\mathrm{GH} 5)$ & $\begin{array}{l}116 \\
\text { Glu-HIS-LEU-PRO-ALA-GLY-Phe }\end{array}$ & Zaire \\
\hline$a 118(H 1)-a 119(H 2)$ & $\begin{array}{lr}118 & 119 \\
\text { Thr-GLU-PHE-THR-Pro }\end{array}$ & Grady (Dakar) \\
\hline$\beta 95(F G 2)-\beta 96(F G 3)$ & $\begin{array}{l}95 \\
\text { LyS-LEU-HIS-LYS-ASP-LYS-Leu }\end{array}$ & Koriyama \\
\hline Insertions/Deletions & & \\
\hline$B 72(E 16)-B 76(E 20)$ & $\begin{array}{l}72 \\
\text { Ser-ALA-ARG-LYS-GLU-A1a }\end{array}$ & Montreal \\
\hline
\end{tabular}


known Hbs (Constant Spring, Icaria, Koya Dora, and Seal Rock) have arisen by a point mutation in the chain termination $C D$ of the $\alpha 2-g l o b i n$ gene $(85-88)$.

Another group of variants with an extension at the C-terminus include four variants that are products of genes carrying a frameshift at the third exon. These variants are $H b$ Wayne (frameshift at $C D$ a 139), Hb Saverne (at CD B143), Hb Cranston (at CD B 145), and Hb Tak (at CD B 146) (89-92). The only a chain variant, Hb Wayne, is the result of a one base deletion (i.e. A) at CD 139 of the $\alpha 2$-globin gene $(89,93)$. Hb Saverne has also arisen from a one base deletion, and its $B$ chain is 156 amino acids long (90). The two other $\beta$ chain variants are the result of two base insertions, that occurs at CD 145 in Hb Cranston (91) and at CD 146 in Hb Tak (2); their sequences beyond the sites of mutation are identical, both being extended by 11 additional amino acids.

Four known variants are elongated at their amino terminus; one is an a chain variant, Hb Thionville (94), and three are $\beta$ chain variants: Hb Long Island-Marseille (95), Hb Doha (96), and Hb South Florida (97). All these variants carry a mutation at $C D 1$ or 2 , and have a methionyl residue preceding the normal valyl residue at position 1 of the $a$ and $B$ chains. Methionine is the first residue to be incorporated, but it is normally cleaved during translation of the nascent polypeptide chain. The substitutions at the first two positions of the polypeptide chain interfere with the enzymatic cleavage step that normally removes the amino terminal methionine (97).

Four abnormal variants have been described that are elongated at sites other than at the carboxy or amino termini. Three are a variants: $\mathrm{Hb}$ Catonsville (98), Hb Zaire (99), and Hb Grady (Dakar) (100), and one is a $\beta$ chain variant, Hb Koriyama (101). Hb Grady was the first variant of this group to be discovered; it has an insertion of three residues between positions 118 and 119 (100). The mechanism responsible for these mutations is an insertion of three or multiple of three nts, that does not impair the reading frame of the mRNA, and leads to the insertion of one or more amino acids.

Hbs With Shortened Polypeptide Chains. Fifteen variants (two a and 13 B have a shorter globin chain due to deletion of one to five amino acid residues (Table 1/4). Thirteen of these are considered to be the result of small deletions in the coding region of the appropriate globin gene, while two are due to a point mutation causing premature translation termination (nonsense mutation). The a chain variant $\mathrm{Hb}$ Natal has arisen by a base pair substitution leading to a stop CD at at the penultimate CD of the $\alpha 2-g l o b i n$ gene (102), and Hb McKees Rocks by an analogous substitution in the $\mathrm{B}-\mathrm{globin}$ gene (103).

Two additional shortened $B$ chain variants, Hbs Birmingham and Galicia are the result of deletions of nine and three nts from the coding sequences

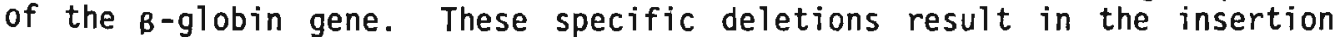
of one amino acid residue in addition to the deletion of four and two amino acids, respectively (104). 
TABLE 1/4. Hbs With Shortened Polypeptide Chains

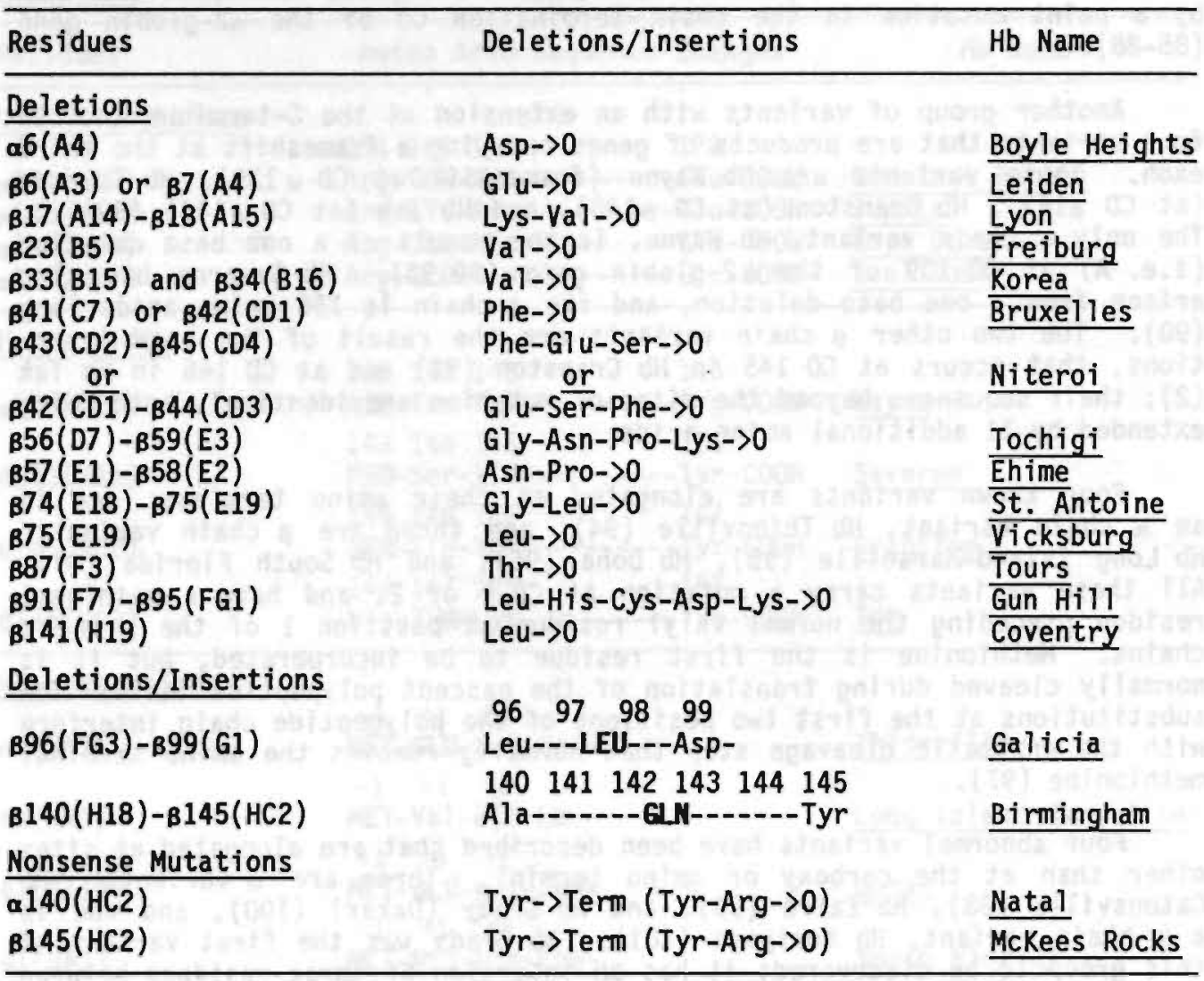

Fusion Hbs. Fusion or hybrid Hbs are variants that have arisen through nonhomologous crossover events between two misaligned chromosomes; nine such Hbs have been described (Table 1/5).

The $\delta \beta$-Lepore gene is a combination of the $5^{\prime}$ end of the $\delta$ gene and the $3^{\prime}$ end of the $B$ gene (105). Three different types of Hb Lepore have been described, in which the transition from $\delta->\beta$ sequence occurs at different points. Most prevalent is $H \mathrm{~b}$ Lepore-Boston, with $\delta$ chain sequence through amino acid position 87 and a $B$ chain sequence starting at position 116 (106). It has been described in different populations. Two other Lepore Hbs, Lepore-Hollandia (107) and Lepore-Baltimore (108) have $\delta B$ chains with a crossover at $\delta 22 / \beta 50$ and $\delta 50 / \beta 86$, respectively. All Lepore Hbs are synthesized at a low level and its carriers exhibit thalassemialike hematological features (105).

The crossover event responsible for the occurrence of the Lepore chromosome gives rise to another abnormal chromosome, the "anti-Lepore" chromosome, that carries normal $\delta$ and $B$ genes as well as a hybrid $B \delta$ gene. Four different anti-Lepore Hbs have been characterized: Hbs Miyada (109), PNilotic (110), P-Congo (111), and Lincoln Park (112). Hb Lincoln Park has 
TABLE 1/5. Fusion $\mathrm{Hbs}^{\mathrm{a}}$

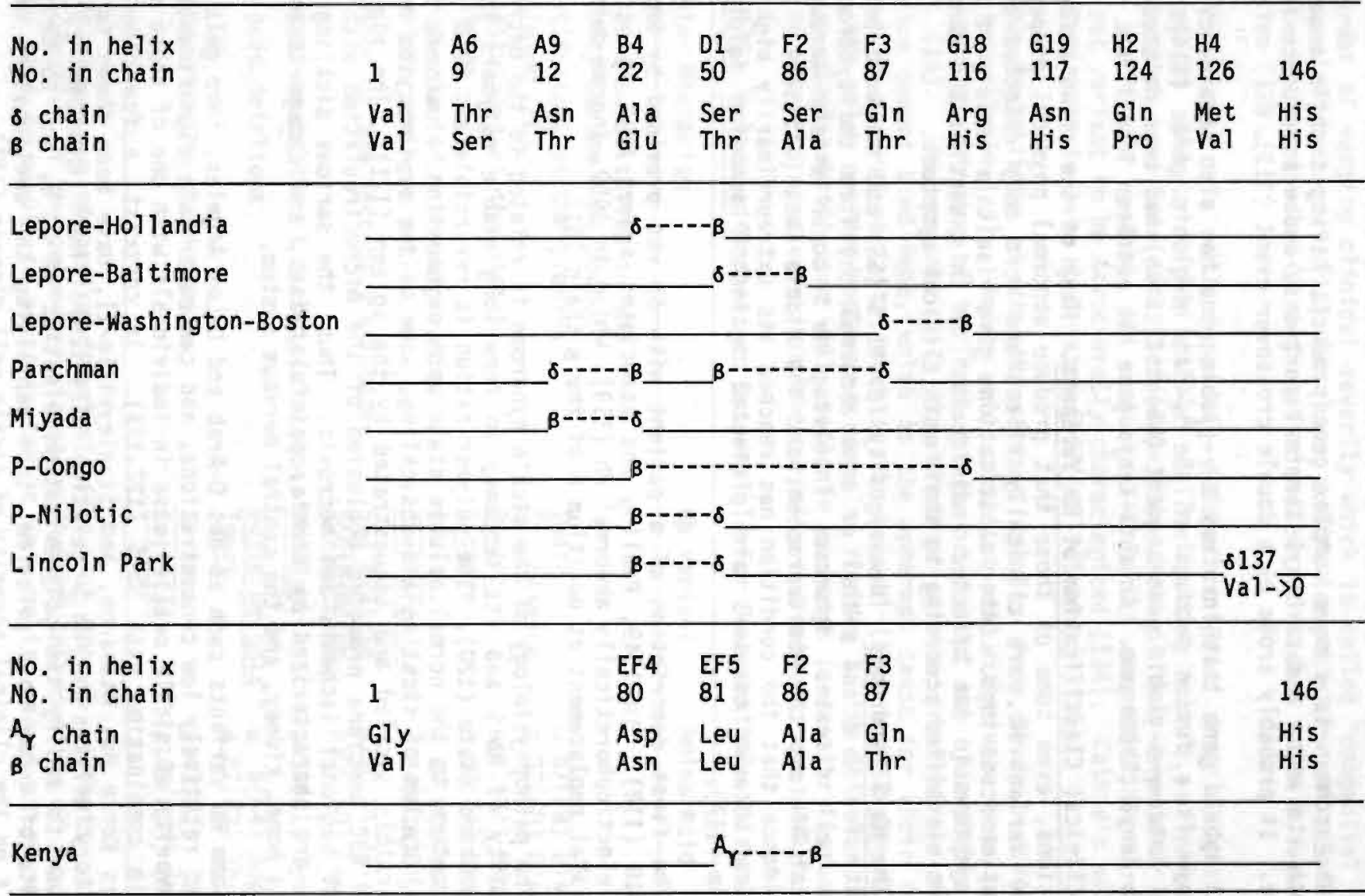

a From Ref. 82. 
the same structural characteristics as $\mathrm{Hb}$ P-Nilotic but in addition, it has a deletion of a valine residue at position $\delta 137$. Because of the presence of a normal $B$ gene in cis, patients with anti-Lepore Hbs are clinically normal (105).

Hb Parchman is a more complex gene product, having $\delta$ chain sequences in both its amino and carboxyl terminal portions, and a $\beta$ sequence in the middle. It probably arose by a double crossover event (113).

A hybrid gene that involves a $r$-globin gene has also been described; $\mathrm{Hb}$ Kenya is a fusion product of the $A_{\gamma^{-}}$and $B-g l o b i n$ genes (114). The

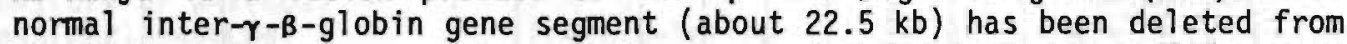
the $\mathrm{Hb}$ Kenya chromosome. An anti-Kenya gene has not been identified.

Clinical Classification of Hb Variants. Many of the amino acid substitutions, even some of those that produce abnormal physical properties in the variant $\mathrm{Hb}$, are clinically silent and were only detected during population screenings. Other substitutions give rise to a variety of clinical symptoms in the affected individuals. In this section the abnormal Hbs are classified according to their main clinical symptoms.

The Hb S Syndromes. Homozygosity for $\mathrm{Hb} \mathrm{S}$ (SS) and compound heterozygosity for $\mathrm{Hb} \mathrm{S}$ and $\mathrm{B}$-thal or some abnormal $\mathrm{Hb}$, form the syndrome of sickle cell disease. Diseases involving $\mathrm{Hb} S$ occur widely throughout tropical Africa, the Mediterranean, and the Middle East $(115,116)$. There is evidence that the condition has reached its extraordinarily high frequencies because carriers are protected against Plasmodium falciparum malaria (117).

The first description of a patient with SS was provided by Herrick in 1910 (118). In 1949, Pauling and associates showed that it was due to an electrophoretically abnormal $\mathrm{Hb}$ (119), while in 1959, Ingram detected a Glu->Val replacement at position 6 of the $B$ chain (81).

The pathophysiology of the sickle syndrome is related to the decreased solubility of $\mathrm{Hb} \mathrm{S}$ and its tendency to form long stable polymers in the deoxygenated state (120). The polymerization is reversible and $\mathrm{Hb} S$ molecules return to the normal soluble state upon oxygenation. However, after several cycles of sickling and unsickling, some of the erythrocytes become irreversibly sickled and sequestrated by the spleen (121). The rigidity of the RBC membrane promotes occlusion of the microcirculation with subsequent regional ischemia and necrosis. Thus, the serious sickling disorders are characterized by anemia, painful crises, and damage to organs such as bone, kidney, and the central nervous system.

Some $H b$ variants such as $H b s$ 0-Arab and D-Los Angeles, form gels with $\mathrm{Hb} S$ at relatively low concentrations, and can contribute significantly to the severity of sickle cell disease in individuals with one of these variants in combination with $\mathrm{Hb} \mathrm{S}(122,123)$. In contrast, a few other Hbs such as Korle Bu, Hasharon, and Stanleyville-II, have been shown to have inhibitory effects on $\mathrm{Hb} S$ gelation (124-126). Most importantly, Hb F is known to modify the polymerization kinetics of $\mathrm{Hb} \mathrm{S}$, and to decrease the rate of deoxy $\mathrm{Hb} S$ gelation, thus explaining the amelioratory effect of $\mathrm{Hb} F$ on the clinical severity of sickle cell disease (127). 
The Unstable Hbs (Hemolytic Anemias). A large group of Hb variants show instability caused by their altered molecular structures. As a result, these Hbs tend to precipitate within the erythrocytes, with the formation of insoluble protein inclusions (Heinz bodies). Patients have a disorder of varying clinical severity which is called "congenital Heinz body hemolytic anemia" (15) and have a life-long history of anemia and jaundice with episodes of aggravation often coincident with a recurrent infection (128).

The first case with "apparent idiopathic Heinz body anemia" was reported in 1952 by Cathie (129). Following this report, a number of similar cases have been described (130-133). Most of them were later found to be due to an unstable Hb variant. Hb Zurich or B 63(E7)His->Arg was the first variant to be structurally characterized (134). Cathie's patient was later shown to have Hb Bristol [B 67(E11)Val->Asp] (135).

The instability of the $\mathrm{Hb}$ variant results from several different mechanisms (Table 1/6). Amino acid substitutions within the heme pocket may impair the binding of the heme group, or disrupt the hydrophobic heme pocket (15). Hb variants with substitution at the proximal or distal histidine cannot bind heme, which is the apparent cause for their instability. In several variants, such as Hbs Bristol (135) and Shepherds Bush (136), the polar residue replaces a nonpolar residue in the interior of the subunit. Such substitution will result in a change in tertiary structure, allowing water to penetrate into the normally hydrophobic heme pocket.

TABLE 1/6. Molecular Mechanisms Responsible For Some Unstable Hb Variants

Molecular Mechanism Hb Variant Amino Acid

Amino acid substitution in the

vicinity of the heme pocket

Substitutions at the proxima: histidine (F8)

Substitutions at the distal

histidine (E7)

Introduction of a nonpolar amino acid into the heme pocket

Disruption of the secondary structure

\begin{tabular}{|c|c|}
\hline$\frac{\text { Iwata }}{\text { St. Etienne }}$ & $\begin{array}{l}\text { a } 87 \text { (F8) His->Arg } \\
\text { B } 92 \text { (F8) His->Glu }\end{array}$ \\
\hline$\frac{\text { Zurich }}{\text { Bicetre }}$ & $\begin{array}{l}\text { B } 63 \text { (E7) His->Arg } \\
\text { B } 63 \text { (E7) His->Pro }\end{array}$ \\
\hline $\begin{array}{l}\text { Bristol } \\
\text { Shepherds Bush }\end{array}$ & $\begin{array}{l}\text { B } 67 \text { (E11) Val->Asp } \\
\text { B } 74 \text { (E18)Gly->Asp }\end{array}$ \\
\hline$\frac{\text { Sun Prairie }}{\text { Southampton }}$ & $\begin{array}{l}\alpha 130(\mathrm{H} 13) \text { Ala->Pro } \\
\text { B106(G8) Leu->Pro }\end{array}$ \\
\hline Philly & B 35 (C1) Tyr->Phe \\
\hline $\begin{array}{l}\text { Boyle Heights } \\
\text { Tours }\end{array}$ & $\begin{array}{lrl}\alpha & 6(A 4) & \text { Asp }->0 \\
\text { B } & 87(F 3) & \text { Th } r->0\end{array}$ \\
\hline$\frac{\text { Saverne }}{\text { Cranston }}$ & $\begin{array}{ll}\beta 143(H 21) & (F S C) \\
\beta 145(H C 2) & (F S C)\end{array}$ \\
\hline
\end{tabular}

Substitutions in the 11 contact region Philly

Amino acid deletions

Elongation of the globin chain

An important mechanism for instability of the Hb variants is also the disruption of the secondary structure through introduction of a proline residue that is unable to participated in an $a$-helix, except as one of the 
initial three residues. Four $\alpha$ and 22 B chain variants with this type of substitution have been detected. Ten of the 22 B chain variants include substitution of proline for leucine. In addition, seven have an Ala- $>$ Pro replacement, six a His->Pro, one a Thr->Pro, and one a Gin->Pro substitution (1). Hb Valletta [B87(F3)Thr->Pro] is a stable variant, since the introduction of a proline residue in the third position of the $F$ helical segment does not disrupt the secondary structure (137).

Molecular instability is also an accompanying feature of Hb variants that have amino acid substitutions at the al 1 l interface, thus allowing dimers to dissociate into monomers; Hb Philly [B 35(C1)Tyr->Phe] is an example of this type of unstable variant (138).

Deletions of amino acid residues alter the primary and secondary structure, as well as the conformation of the $\mathrm{Hb}$ molecule, and most of these variants are unstable. Finally, globin chain elongation can result in instability due to hydrophobic properties of the extended chain, i.e. Hbs Cranston and Saverne (139).

Abnormal Hbs Hith High 0 ? Affinity (Erythrocytosis). One group of $\mathrm{Hb}$ variants has amino acid substitutions that increase the affinity of $\mathrm{Hb}$ for $\mathrm{O}_{2}$. Some of these variants cause erythrocytosis in the carriers. The first such variant detected was Hb Chesapeake which was found in an 81year-old man, who was in essentially good health but had a markedly elevated $\mathrm{Hb}$ level (140). Most of the patients with a high affinity Hb type and with erythrocytosis are asymptomatic, except for the occasional presence of a ruddy complexion. Most commonly, erythrocytosis is diagnosed during a routine hematological examination. However, and individual with $\mathrm{Hb}$ Malmo, who had experienced symptoms of severe cardiac dysfunction, has been reported; he has benefited from phlebotomy and the transfusion of normal blood (141).

The major reason for an increased $\mathrm{O}_{2}$ affinity is a structural alteration that affects the equilibrium between the $T$ and $R$ quaternary conformations of the $H b$ tetramer. Amino acid substitutions that decrease the stability of the $T$ structure and promote the $R$ state will increase the affinity of the $\mathrm{Hb}$ molecule. Most of the high affinity Hbs have mutations in the binding sites of 2,3-DPG, in the carboxy terminus of the $B$ chain, and at the a1B2 interface, all being important regions for regulating the function of the $\mathrm{Hb}$ molecule (1).

An example is Hb Kempsey, one of the particularly well-studied high $0_{2}$ variants with an amino acjd replacement at the al $B 2$ interface $(142,143)$. The substitution, $B 99(G 1) A s p->A s n$, inhibits the formation of the most important hydrogen bond between a $42 \mathrm{Tyr}$ and $\mathrm{B} 99 \mathrm{Asp}$, that stabilizes the T structure.

Hb Hiroshima is a high affinity variant with a mutation at the C-terminal end of the $B$ chain; the histidine residue is replaced by aspartic acid at position $B 146$ (144). All variants that affect the $B 146 \mathrm{His}$ also have a reduced Bohr effect, thus confirming the identification of this residue as a major source of the alkaline Bohr effect (20). 
Hb Helsinki [B 82(EF6)Lys->Met] and Hb Syracuse [B143(H21)His->Pro] are examples of high affinity variants that have substitutions at sites responsible for binding of 2,3-DPG $(145,146)$.

The Hb M Variants (Congenital Cyanosis). Because of their distinctive physical appearance, individuals affected by one of the Hb $M$ variants can be readily detected. They have cyanosis but are otherwise totally asymptomatic (15). The German physicians Horlein and Weber provided the first convincing evidence of a familial hemoglobinopathy in 1948 (147). They described a family with carriers of a congenital cyanotic syndrome and proved that the genetic defect resides in the globin and not in the heme. The structural analysis of $M$ Hbs was first completed in 1961 when three Hb $M$ variants were characterized: Hb M-Boston [a 58(E7)His->Tyr], Hb MSaskatoon [B63(E7)His-Tyr], and Hb M-Milwaukee-I [B67(E1I)Val->Glu] (148). All of the $H b M$ variants have amino acid replacements at positions that are in close proximity to the heme groups, thus stabilizing the heme iron atoms in the ferric $\left(F^{+3}\right)$ state. The iron in the abnormal subunit is therefore incapable of binding $\mathrm{O}_{2}$. Most of the $\mathrm{Hb} \mathrm{M}$ variants also have low affinity for $\mathrm{O}_{2}$. In four of the five $M$ Hbs, involving either a or $\beta$ chains, the distal or the proximal histidine is replaced by tyrosine (148-150). In the fifth, Hb Milwaukee-I, Val 67 is replaced by glutamic acid; the longer side chain of this amino acid can reach and perturb the heme iron (151). The two known fetal $H b$ $M$ variants are $H b F-M-0$ saka $\left[{ }^{G}{ }^{6} 3\right.$ (E7)His->Tyr] and Hb F-M-Fort Ripley [G ${ }^{\text {Y } 92(F 8) H i s->T y r] ~}(152,153)$.

Abnonmal Hbs With Low 0 ? Affinity. A number of $\mathrm{Hb}$ variants are characterized by a lower than normal affinity for $0_{2}$. Some of the unstable $\mathrm{Hb}$ variants and some of the $M$ Hbs also have decreased $\mathrm{O}_{2}$ affinity. Individuals with $\mathrm{Hb}$ variants that have significantly low $\mathrm{O}_{2}$ affinity exhibit a slate gray color involving the skin and mucous membranes (15). Hb Kansas [B 102(G4)ASn->Thr] was the first of these variants to be identified and is the most thoroughly studied $(154,155)$. The amino acid substitution at B102 in $\mathrm{Hb}$ Kansas prevents the formation of alB2 hydrogen bonds between a94 Asp and B102 Asn that normally stabilizes the R quaternary structure. This change shifts the R-T equilibrium in favor of the low $\mathrm{O}_{2}$ affinity $T$ quaternary form. Low affinity for $\mathrm{O}_{2}$ and cyanosis in the carriers have been encountered in two other $\mathrm{Hb}$ variants with a substitution at B102, i.e. Hb Beth Israe] (->Ser) (156) and Hb Saint Mande (->Tyr) (157).

\section{REFERENCES}

1. Huisman, T.H.J.: The structure and function of normal and abnormal haemoglobins. In Bailliere's Clinical Haematology, edited by D.J. Weatherall and D.R. Higgs, Vo1. 6, page 1, W.B. Saunders Company, London, 1993.

2. Konigsberg, W., Guidotti, G., and Hill, R.J.: The amino acid sequence of the a chain of human hemoglobin. J. Biol. Chem., 236:55, 1961.

3. Goldstein, J., Konigsberg, W., and Hill, R.J.: The structure of human hemoglobin. VI. The sequence of amino acids in the tryptic peptides of the B chain. J. Biol. Chem., 238:2016, 1963. 
4. Jones, R.T.: Structural studies of aminoethylated hemoglobins by automatic peptide chromatography. Cold Spring Harbor Symp. Quant. Biol., 29:297, 1964.

5. Schroeder, W.A., Shelton, J.R., Shelton, J.B., Cormick, J., and Jones, R.T.: The amino acid sequence of the $Y$ chain of human fetal hemoglobin. Biochemistry, 2:992, 1963.

6. Schroeder, W.A., Huisman, T.H.J., Shelton, J.R., Shelton, J.B., Kleihaver, E.F., Dozy, A.M., and Robberson, B.: Evidence for the multiple structural genes for the $Y$ chain of human fetal hemoglobin. Proc. Nat1. Acad. Sci. USA, 60:537, 1968.

7. Clegg, J.B.: Embryonic hemoglobin: Sequence of the $\varepsilon$ and $\zeta$ chains. In Human Hemoglobins and Hemoglobinopathies; A Review to 1981, Texas Reports on Biology and Medicine, edited by R.G. Schneider, S. Charache, and W.A. Schroeder, Vol. 40, page 23, The University of Texas Medical Branch, Galveston, TX, 1980-1981.

8. Perutz, M.F.: Structure and mechanism of haemoglobin. Br. Med. Bul1., 32:195, 1976.

9. Perutz, M.F.: X-ray analysis of hemoglobin. Science, 140:863, 1963.

10. Perutz, M.D., Kendrew, J.C., and Watson, H.C.: Structure and function of hemoglobin. II. Some relations between polypeptide chain configuration and amino acid sequence. J. Mol. Biol., 13:669, 1965.

11. Perutz, M.F. and Lehmann, H.: Molecular pathology of human haemoglobin. Nature, 219:902, 1968.

12. Fermi, G., Perutz, M.F., Shaanan, B., and Fourme, R.: The crystal structure of human deoxyhemoglobin at $1.74 \AA$ resolution. J. Mol. Biol., 175:159, 1984.

13. Perutz, M.F.: Stereochemistry of cooperative effects in haemoglobin. Nature, 228:726, 1970.

14. Fermi, G. and Perutz, M.F.: Haemoglobin and myoglobin. In Atlas of Molecular Structure in Biology, edited by D.C. Philips and F.M. Richards, Clarendon Press, 0xford, 1981.

15. Bunn, H.F. and Forget, B.G.: Hemoglobin: Molecular, Genetic and Clinical Aspects, W.B. Saunders Company, Philadelphia, 1986.

16. Lehmann, H. and Casey, R.: Human haemoglobin. Comp. Biochem., 18: $347,1982$.

17. Arnone, A.: X-ray diffraction study of binding of 2,3-diphosphoglycerate to human deoxyhaemoglobin. Nature, 237:146, 1972.

18. Perutz, M.F., Fermi, G., Abraham, D.J., Poyart, C., and Bursaux, E.: Hemoglobin as a receptor of drugs and peptides: $X$-ray studies of the stereochemistry of binding. J. Am. Chem. Soc., 108:1064, 1986.

19. Shaanan, B.: The crystal structure of oxyhaemoglobin at $2.1 \AA$ resolution. J. Mol. Biol., 171:31, 1983.

20. Perutz, M.F.: Molecular anatomy, physiology, and pathology of hemoglobin. In The Molecular Basis of Blood Diseases, edited by $G$. Stamatoyannopoulos, A.W. Nienhuis, P. Leder, and P.W. Majerus, page 127, W.B. Saunders Company, Philadelphia, 1987.

21. Reeves, R.B.: The effect of temperature on the oxygen equilibrium curve of human blood. Respir. Physiol., 42:317, 1980.

22. Nage1, R.L.: Genetically Abnormal Red Cells, CRC Press, Inc., Boca Raton, FL, 1988.

23. Bohr, C.: Theoretische Behandlung der quantitativen Verhaltius bei der Sauerstoffaufnahine des Hemoglobins. Zbl. Physiol., 17:682, 1904. 
24. Perutz, M.F., Kilmartin, J.V., Nishikura, K., Fogg, J.H., Butler, P.J.G., and Rollema, H.S.: Identification of residues contributing to the Bohr effect of human hemoglobin. J. Mol. Biol., 138:649, 1980 .

25. Kilmartin, J.V., Fogg, J.H., and Perutz, M.F.: Role of C-terminal histidine in the alkaline Bohr effect of human hemoglobin. Biochemistry, 19:3189, 1980 .

26. Perrela, M., Kilmartin, J.V., Fogg, J., and Rossi-Bernardi, L.: Identification of the high and $1 \mathrm{OW}$ affinity $\mathrm{CO}_{2}$-binding sites of human haemoglobin. Nature, 256:759, 1975.

27. Bauer, C. and Schroeder, E.: Carbamino compounds of haemoglobin in human adult and foetal blood. J. Physiol. London, 227:457, 1972.

28. Chanutin, A. and Curnish, R.R.: Effect of organic and inorganic phosphates on the oxygen equilibrium of human erythrocytes. Arch. Biochem. Biophys., 121:96, 1967.

29. Benesch, R. and Benesch, R.E.: The effect of organic phosphates from the human erythrocyte on the allosteric properties of hemoglobin. Biochem. Biophys. Res. Commun., 26:162, 1967.

30. Benesch, R., Benesch, R.E., and Yu, C.I.: Reciprocal binding of oxygen and diphosphoglycerate by human hemoglobin. Proc. Natl. Acad. Sci. USA, 59:526, 1968.

31. Perutz, M.F.: The Bohr effect and combination with organic phosphates. Nature, 228:734, 1970.

32. Tyuma, I. and Shimizu, K.: Different response to organic phosphates of human fetal and adult hemoglobins. Arch. Biochem. Biophys., 129: $404,1969$.

33. Maurer, H.S., Behrman, R.E., and Honig, G.R.: Dependence of the oxygen affinity of blood on the presence of foetal and adult haemoglobin. Nature, 227:388, 1970.

34. van Beek, G.G.M. and de Bruin, S.H.: Identification of the residues involved in the oxygen-linked chloride ion binding sites in human deoxyhemoglobin and oxyhemoglobin. Eur. J. Biochem., 105:353, 1980.

35. Deisseroth, A., Nienhuis, A., Turner, P., Velez, R., Anderson, W.F., Ruddle, F., Lawrence, J., Creagan, R., and Kucherlapati, R.: Localization of the human $\alpha-g l o b i n$ structural gene to chromosome 16 in somatic cell hybrids by molecular hybridization assay. Cel1, 12:205, 1977.

36. Koeffler, H.P., Sparkes, R.S., Stang, H., and Mohondas, T.: Regional assignment of genes for human a-globin and phosphoglycollate phosphate to the short arm of chromosome 16. Proc. Natl. Acad. Sci. USA, 78:7015, 1981 .

37. Deisseroth, A., Nienhuis, A., Lawrence, J., Giles, R., Turner, P., and Ruddle, F.H.: Chromosomal localization of human $\beta$-globin gene on human chromosome 11 in somatic cell hybrids. Proc. Nat1. Acad. Sci. USA, 75:1456, 1978.

38. Lebo, R.V., Carrano, A.V., Burkhart-Shultz, K., Dozy, A.M., Yu, L-C., and Kan, Y.W.: Assignment of human $B, \gamma$, and $\delta$-globin genes to the short arm of chromosome 11 by chromosome sorting and DNA restriction enzyme analysis. Proc. Nat1. Acad. Sci. USA, 76:5804, 1978.

39. Lauer, J., Shen, C.K., and Maniatis, T.: The chromosomal arrangement of human a-like globin genes: Sequence homology and $\alpha-g l o b i n g e n$ deletions. Cel1, 20:119, 1980. 
40. Fritsch, E.F., Lawn, R.M., and Maniatis, T.: Molecular cloning and characterization of the human B-like globin gene cluster. Cell, $19: 959,1980$.

41. Marks, J., Shaw, J.P., and Shen, C.K.: Sequence organization and genomic complexity of primate el-globin gene, a novel a-like gene. Nature, 321:785, 1968.

42. Lawn, R.M., Efstratiadis, A., O'Connell, C., and Maniatis, T.: The nucleotide sequence of the huma B-globin gene. Ce11, 21:647, 1980.

43. Liebhaber, S.A., Goossens, M.J., and Kan, Y.W.: Cloning and complete

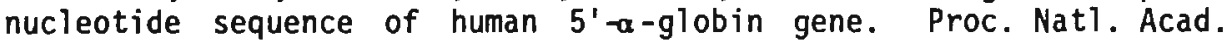
Sci. USA, 77:7054, 1980 .

44. Spritz, R.A., deRiel, J.K., Forget, B.G., and Weissman, S.M.: Complete nucleotide sequence of the human $\delta$-globin gene. Cell, 21:639, 1980.

45. Slightom, J.L., Blechl, A.E., and Smithies, 0.: Human fetal G $\mathrm{G}_{\gamma-\text { and }}$ A ${ }^{-g l o b i n}$ gene: Complete nucleotide sequence suggest that DNA can be exchanged between these duplicated genes. Ce11, 21:627, 1980.

46. Baralle, F.E., Shoulders, C.C., and Proudfoot, N.J.: The primary structure of the human $\varepsilon-g l$ obin gene. Cell, 21:621, 1980.

47. Proudfoot, N.J., Gil, A., and Maniatis, T.: The structure of the human zeta-globin gene and a closely linked, nearly identical pseudogene. Cel1, 31:552, 1982 .

48. Efstratiadis, A., Posakony, J.W., Maniatis, T., Lawn, R.M., O'Conne11, C., Spritz, R.A., deRiel, J.K., Forget, B.G., Weissman, S.M., Slightom, J.L., Blech1, A.E., Smithies, 0., Baralle, F.E., Shoulders, C.C., and Proudfoot, N.J.: The structure and evolution of the human B-globin gene family. Cell, 21:653, 1980 .

49. Dierks, P., van Ooyen, A., Cochran, M.D., Dobkin, C., Reiser, J., and Weissman, C.: Three regions upstream from the Cap site are required for efficient and accurate transcription of the rabbit $\beta$ globin gene in mouse 3TG cells. Cell, 32:695, 1983.

50. Eaton, W.A.: The relationship between coding sequences and function of haemoglobin. Nature, $284: 183,1980$.

51. Go, M.: Correlation of DNA exonic regions with protein structural units of haemoglobin. Nature, 291:90, 1981.

52. Nienhuis, A.W. and Maniatis, T.: Structure and expression of globin genes in erythroid cells. In The Molecular Basis of Blood Diseases, edited by G. Stamatoyannopoulos, A.W. Nienhuis, P. Leder, and P.W. Majerus, page 28, W.B. Saunders Company, Philadelphia, 1987.

53. Breathnach, R., Benoist, C., O'Hare, K., Gannon, F., and Chambon, P.: Ovalbumin gene: Evidence for a leader sequence in mRNA and DNA sequences of the exon-intron boundaries. Proc. Natl. Acad. Sci. USA, $75: 4853,1978$.

54. Karisson, S. and Nienhuis, A.W.: Developmental regulation of human globin genes. Ann. Rev. Biochem., 54:1071, 1985.

55. Weathera11, D.J., Wood, W.G., Jones, R.W., and Clegg, J.B.: The developmental genetics of human hemoglobin. In Progress in Clinical and Biological Research, edited by G. Stamatoyannopoulos and A.W. Nienhuis, Experimental Approaches for the Study of Hemoglobin Switching, Vol. 191, page 3, Alan R. Liss, Inc., New York, 1985.

56. Stamatoyannopoulos, G. and Nienhuis, A.W.: Hemoglobin switching. In The Molecular Basis of Blood Diseases, edited by G. Stamatoyanno- 
poulos, A.W. Nienhuis, P. Leder, and P.W. Majerus, page 66, W.B. Saunders Company, Philadelphia, 1987.

57. Berg, P.E. and Schechter, A.N.: The impact of molecular biology on the diagnosis and treatment of hemoglobin disorders. Mol. Genet. Med., 2:1, 1992 .

58. Orkin, S.H.: Globin gene regulation and switching. Cell, 63:665, 1990.

59. Huehns, E.R., Dance, N., Beaven, G.H., Keil, J.V., Hecht, F., and Motulsky, A.G.: Human embyronic haemoglobins. Nature, 201:1095, 1964.

60. Huehns, E.R. and Beaven, G.H.: Developmental changes in human hemoglobins. Clin. Dev. Med., 37:175, 1971.

61. Huehns, E.R. and Farooqui, A.M.: 0xygen dissociation properties of human embryonic red cells. Nature, 254:335, 1975.

62. Capp, G.L., Rigas, D.A., and Jones, R.T.: Evidence for a new haemoglobin chain ( $\zeta$ chain). Nature, 288:278, 1970.

63. Randhawa, Z.I., Jones, R.T., and Lie-Injo, L.E.: Human Hemoglobin Portland II $\left(c_{S_{B}} 2\right)$ : Isolation and characterization of Portland hemoglobin components and their constituent chains. J. Biol. Chem., $259: 7325,1984$.

64. Kutlar, F., Moscoso, H., Kiefer, C.R., Garver, F.A., Beksac, S., Onderoglu, L., Gurgey, A., Altay, C., and Huisman, T.H.J.: The quantities of adult, fetal, and embryonic globin chains in the blood of 18 to 20-week-old fetuses. J. Chromatogr., 567:359, 1991.

65. Kutlar, F., Fei, Y.J., Wilson, J.B., Kutlar, A., and Huisman, T.H.J.: Detection of the embryonic $\zeta$ chain in the blood from newborn babies from different countries and in babies with some significant hemoglobinopathies. Acta Haematol., 78:28, 1987.

66. Kutlar, F., Gonzalez-Redondo, J.M., Kutlar, A., Gurgey, A., Altay, C., Efremov, G.D., Kleman, K., and Huisman, T.H.J.: The levels of $\zeta, Y$, and $\delta$ chains in patients with $\mathrm{Hb} H$ disease. Hum. Genet., 82 : $179,1989$.

67. Kutlar, F., Reese, A.L., Hsia, Y.E., Kleman, K.M., and Huisman, T.H.J.: The types of hemoglobin and globin chains in hydrops fetalis. Hemoglobin, 13:671, 1989.

68. Epstein, M., Fuchaoren, S., Gu, L-H., Huisman, T.H.J., Moore, R., Ugarte, E., and Epstein, N.: Detection of the embryonic $\zeta$ chain in red cells of adults with a variety of a-globin gene deficiencies. Abstract, B1ood, 78:782, 1991.

69. Von Koerber, E.: Inaugural dissertation: "Uber differenzen Bluffarbstoffes". Dorpat, 1866.

70. Kutlar, A., Kutlar, F., Gu, L-G., Mayson, S.M., and Huisman, T.H.J.: Fetal hemoglobin in normal adults and $B$-thalassemia heterozygotes. Hum. Genet., 85:106, 1990.

71. Grifoni, V., Kamuzora, H., Lehmann, H., and Charlesworth, D.: A new $\mathrm{Hb}$ variant: Hb F Sardinia Y75 (EI9) isoleucine-sthreonine found in a family with Hb G Philadelphia, B-chain deficiency and a Lepore-like haemoglobin indistinguishable from $\mathrm{Hb} \mathrm{A}_{2}$. Acta Haematol., 53:347, 1975.

72. Schroeder, W.A., Huisman, T.H.J., Efremov, G.D., Shelton, J.R., Shelton, J.B., Phillips, R., Reese, A., Gravely, M., Harrison, J.M., and Lam, H.: Further studies of the frequency and significance of the $T_{Y}$-chain of heman fetal hemoglobin. J. Clin. Invest., 63:268, 1979. 
73. Huisman, T.H.J., Kutlar, F., and Gu, L-H.: $\gamma$ Chain abnormalities and $\gamma$-globin gene rearrangements in newborn babies of various populations. Hemoglobin, 15:349, 1991.

74. Schroeder, W.A., Cua, J.T., Matsuda, G., and Fenninger, W.D.: Hemoglobin $F_{1}$, and acetyl containing hemoglobin. Biochim. Biophys. Acta, 63:532, 1962 .

75. Huisman, T.H.J., Altay, C., Webber, B., Reese, A.L., Gravely, M.E., Okonjo, K., and Wilson, J.B.: Quantitation of three types of $\gamma$ chain of $\mathrm{Hb} F$ by high pressure liquid chromatography: Application of this method to the $\mathrm{Hb} F$ of patients with sickle cell anemia or the S-HPFH condition. Blood, $57: 75,1981$.

76. Gilman, J.G. and Huisman, T.H.J.: DNA sequence variation associated with elevated fetal $G_{\gamma}$ globin production. Blood, 66:783, 1985.

77. Gilman, J.G., Kutlar, F., Johnson, M.E., and Huisman, T.H.J.: A $\mathbf{G}$ to A nucleotide substitution 161 base pairs $5^{\prime}$ of the $G_{\gamma}^{\prime}$ globin gene Cap site $(-161)$ in a high $G_{Y}$ non-anemic person. In Progress in Clinical and Biological Research, edited by G. Stamatoyannopoulos and A.W. Nienhuis, Developmental Control of Globin Gene Expression, Vol. 251, page 415, Alan R. Liss, Inc., New York, 1987.

78. Bunn, H.F., Haney, D.M., Gabbay, K.H., and Gallop, P.M.: Further identification of the nature and linkage of carbohydrate in Hemoglobin $A_{1 c}$. Biochem. Biophys. Res. Commun., 67:103, 1975.

79. Bunn, H.F., Gabbay, K.H., and Gallop, P.M.: The glycosylation of hemoglobin: Relevance to diabetes mellitus. Science, 200:21, 1978.

80. Kunkel, H.G. and Wallenius, G.: New hemoglobin in normal adult blood. Science, 122:288, 1955.

81. Ingram, V.M.: Abnormal human haemoglobins. III. The chemical difference between normal and sickle-cell haemoglobins. Biochim. Biophys. Acta, 36:402, 1959.

82. International Hemoglobin Information Center. Hemoglobin, 17:89, 1993.

83. Bookchin, R.M., Nagel, R.L., and Ranney, H.M.: Structure and properties of Hemoglobin C Harlem, a human hemoglobin variant with amino acid substitutions in 2 residues of the B-polypeptide chain. J. Biol. Chem., 242:248, 1967.

84. Winter, W.P.: Hemoglobin Variants in Human Populations, CRC Press, Inc., Boca Raton, FL, 1987.

85. Clegg, J.B., Weatheral1, D.J., and Milner, P.F.: Haemoglobin Constant Spring - A chain termination mutant? Nature, 234:337, 1971.

86. Clegg, J.B., Weatherall, D.J., Contopolou-Griva, I., Caroutsos, K., Poungouras, P., and Tsevrenis, H.: Haemoglobin Icaria, a new chaintermination mutant which causes a thalassaemia. Nature, 251:245, 1974.

87. De Jong, W.W.W., Meera Khan, P., and Bernini, L.F.: Hemoglobin Koya Dora: High frequency of a chain termination mutant. Am. J. Hum. Genet., $27: 81,1975$.

88. Merritt, D., Jones, R.T., Head, C., Shih, D., Xiao, H., Thibodeau, S.N., and Fairbanks, V.F.: Hb Seal Rock: An extended a chain variant associated with anemia microcytosis and $\alpha$-thalassemia-2 $(-3.7$ $\mathrm{kb})$. Hemoglobin, in press.

89. Seid-Akhaven, M., Winter, W.P., Abramson, R.K., and Rucknagel, D.L.: Hemoglobin Wayne: A frameshift mutation detected in human hemoglobin alpha chains. Proc. Natl. Acad. Sci. USA, 73:882, 1976. 
90. Delanoe-Garin, J., Blouquit, Y., Arous, N., Kister, J., Poyart, C., North, M.L., Bardakdjian, J., Lacombe, C., Rosa, J., and Galacteros, F.: Hemogiobin Saverne: A new variant with elongated $B$ chains: Structural and functional properties. Hemoglobin, 12:337, 1988.

91. Bunn, H.F., Schmidt, G.J., Haney, D.N., and Dluhy, R.G.: Hemoglobin Cranston, an unstable variant having an elongated $B$ chain due to a non-homologous crossover between two normal $\boldsymbol{B}$ chain genes. Proc. Nat1. Acad. Sci. USA, 72:3609, 1975.

92. Flatz, G., Kinderlerer, J.L., Kilmartin, J.V., and Lehmann, H.: Haemoglobin Tak: A variant with additional residues at the end of the $\beta$-chains. Lancet, $i 1: 732,1971$.

93. Salkie, M.L., Higgins, T., Morrison, D.M., Wilson, J.B., Gu, L-H., Suruk, M.A., Baysal, E., and Huisman, T.H.J.: A Canadian family with Hb Wayne; characterization by HPLC and DNA sequencing. Hemoglobin, 16:515, 1992.

94. Vasseur, C., Guillemin, C., Galacteros, F., and Wajcman, H.: Hemoglobin Thionville: An alpha chain variant with substitution of a glutamic residue for valine $\mathrm{NA}-1$ and having an extended $\mathrm{N}$-terminus. Blood, 76:78a (Supp1. 1), 1990.

95. Blouquit, Y., Lena-Russo, G., Delanoe, J., Arous, N., Bardakdjian, J., Lacombe, C., Vovan, L., Orsini, A., Rosa, J., and Galacteros, F.: Hb Marseille a2B2 1 (A1) $\mathrm{NH}_{2}-\mathrm{Met}, 2$ (A2) His->3 (A3) Pro: First variant having a $\mathrm{N}$-terminal elongated $\mathrm{B}$ chain. Blood, 64:55a (Suppl. 1), 1984 .

96. Kamel, K., El-Najjar, A., Webber, B.B., Chen, S.S., Wilson, J.B., and Huisman, T.H.J.: Hb Doha or $\alpha_{2} B_{2}[X-N-M e t-1$ (NA1)Val->Glu]; a new B chain abnormal hemoglobin observed in a Qatari female. Biochim. Biophys. Acta, $831: 257,1985$.

97. Boissel, J-P., Kasper, T.J., Shah, S.C., Malone, J.I., and Bunn, H.F.: Amino-terminal processing of proteins: Hemoglobin South Florida, a variant with a retention of inititator methionine and $\mathrm{N}_{\alpha}$-acetylation. Proc. Nat1. Acad. Sci. USA, 82:8448, 1985.

98. Moo-Penn, W.F., Swan, D.C., Hine, T.K., Baine, R.M., Jue, D.L., Benson, J.M., Johnson, M.H., Virshup, D.M., and Zinkham, H.H.: Hb Catonsville (glutamic acid inserted between Pro-37(C2) $\alpha$ and Thr-38 (C3) a) - Nonallelic gene conversion in the globin system? J. Biol. Chem., 264:21454, 1989.

99. Wajcman, H., Blouquit, Y., Vasseur, C., Le Querrec, A., Melevendi, C., Rasore, A., and Galacteros, F.: Two new human hemoglobin variants caused by unusual mutational events: Hb Zaire contains a five residue repetition within the -chain and $H b$ Duino has two residues substituted in the -chain. Hum. Genet., 78:676, 1992.

100. Huisman, T.H.J., Wilson, J.B., Gravely, M., and Hubbard, M.: Hemoglobin Grady: The first example of a variant with elongated chains due to an insertion of residues. Proc. Nat1. Acad. Sci. USA, 71: $3270,1974$.

101. Kawata, R., Ohba, Y., Yamamoto, K., Miyaji, T., Makita, R., Ohga, K., Watanabe, S., and Miwa, S.: Hyperunstable Hemoglobin Koriyama anti-Hb Gun Hill insertion of five residues in the $\beta$ chain. Hemoglobin, 12:311, 1988 .

102. Jogessar, V.B., Westermeyer, K., Webber, B.B., Wilson, J.B., Hu, H., Gonzalez-Redondo, J.M., Kutlar, A., and Huisman, T.H.J.: Hb Nata 1 


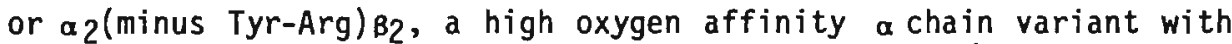
a deleted carboxy-terminus resulting from a TAC->TAA (Tyr->terminating codon) mutation in codon al40. Biochim. Biophys. Acta, 951:36, 1988.

103. Winslow, R.M., Swenberg, M-L., Gross, E., Chervenick, P.A., Buchman, R.R., and Anderson, W.F.: Hemoglobin Mckees Rocks ( $\alpha 2 \beta 2145$ Tyr-) Term): A human "nonsense" mutation leading to a shortened $\beta$-chain. J. Clin. Invest., $57: 772,1976$.

104. Wilson, J.B., Webber, B.B., Hu, H., Kutlar, A., Kutlar, F., Codrington, J.F., Prchal, J.T., Hal, K.M., de Pablos, J.Ma., Rodriguez, I., and Huisman, T.H.J.: Hb Birmingham and Hb Galicia: Two unstable B chain variants characterized by small deletions and insertions. Blood, 75:1883, 1990.

105. Efremov, G.D.: Hemoglobins Lepore and anti-Lepore. Hemoglobin, $2: 197,1978$.

106. Baglioni, C.: The fusion of two peptide chains in Hemoglobin Lepore and its interpretation as a genetic deletion. Proc. Nati. Acad. Sci. USA, 48:1880, 1962.

107. Barnabas, J. and Muller, C.J.: Haemoglobin Lepore Hollandia. Nature, 194:931, 1962.

108. Ostertag, W. and Smith, E.W.: Hemoglobin-Lepore Baltimore, a third type of a $\delta$ B crossover $(\delta 50, \beta 86)$. Eur. J. Biochem., 10:371, 1969.

109. Ohta, Y., Yamaoka, K., Sumida, I., and Yanase, T.: Hemoglobin Miyada, a $\beta-\delta$ fusion peptide (anti-Lepore) type discovered in a Japanese family. Nature New Biol., 234:218, 1971.

110. Badr, F.M., Lorkin, P.A., and Lehmann, H.: Haemoglobin P-Nilotic:

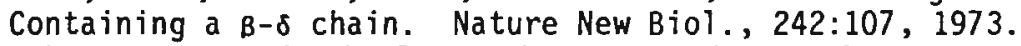

111. Lehmann, H. and Charlesworth, D.: Observation on Haemoglobin $P$ (Congo type). Biochem. J., 119:43, 1970.

112. Honig, G.R., Shamsuddin, M., Mason, R.G., and Vida, L.N.: Hemoglobin Lincoln Park: A $\delta B$ fusion (anti-Lepore) variant with an amino acid deletion in the $\delta$ chain-derived segment. Proc. Natl. Acad. Sci. USA, $75: 1475,1978$.

113. Adams, J.G., III, Morrison, W.T., and Steinberg, M.H.: Hemoglobin Parchman: Double crossover within a single human gene. Science, 218:291, 1982 .

114. Huisman, T.H.J., Wrightstone, R.N., Wilson, J.B., Schroeder, W.A., and Kendall, A.G.: Hemoglobin Kenya, the product of fusion of $Y$ and B polypeptide chains. Arch. Biochem. Biophys., 153:850, 1972.

115. Nagel, R.L. and Ranney, H.M.: Genetic epidemiology of structural mutations of the B-globin gene. Semin. Hematol., 27:342, 1990.

116. Adekile, A.D.: Anthropology of the ${ }_{B} S$ gene-flow from West Africa to North Africa, the Mediterranean, and Southern Europe. Hemoglobin, $16: 105,1992$.

117. Weatherall, D.J.: Common genetic disorders of the red cell and the malaria hypothesis. Ann. Trop. Med. Parasitol., 81:539, 1987.

118. Herrick, J.B.: Peculiar elongated and sickle-shaped red blood corpuscles. Arch. Intern. Med., 20:517, 1910.

119. Pauling, L., Itano, H.A., Singer, S.J., and Wells, I.C.: Sickle cell anemia, a molecular disease. Science, 110:543, 1949.

120. May, A. and Huehns, E.R.: The mechanisms and prevention of sickling. Br. Med. Bull., 32:223, 1976. 
121. Dean, J. and Schechter, A.N.: Sickle cell anemia: Molecular and cellular basis of therapeutic approaches. (Three parts) N. Engl. J. Med., 299:752, 1979.

122. Milner, P.F., Miller, C., Grey, R., Seakins, M., DeJong, W.W., and Went, L.N.: Hemoglobin O-Arab in four Negro families and its interaction with Hemoglobin S and Hemoglobin C. N. Engl. J. Med., 283: $1417,1970$.

123. McCurdy, P.R.: Clinical and physiological studies in a Negro with sickle cel1 Hemoglobin D disease. N. Engl. J. Med., 262:961, 1960.

124. Bookchin, R.M., Nagel, R.L., and Ranney, H.M.: The effect of $B$ 73Ásn on the interaction of sickling hemoglobins. Biochim. Biophys. Acta, $221: 373,1970$.

125. Benesch, R.E., Kwong, S., and Benesch, R.: The effect of a chain mutations $\mathrm{cis}$ and trans to the $\beta 6$ mutation on the polymerization of sickle cell haemoglobin. Nature, 299:231, 1982.

126. Rhoda, M.D., Martin, J., Blouquit, Y., Garel, M.C., Edelstein, S.J., and Rosa, J.: Sickle cell hemoglobin fiber formation strongly inhibited by the Stanleyville-II mutation (a78 Asn->Lys). Biochem. Biophys. Res. Commun., 111:8, 1983.

127. Nage1, R.L., Bookchin, R.M., Johnson, J., Labie, D., Wajcman, H., Issacs-Sodeye, W.A., Honig, G.R., Schiliro, G., Crookston, J.H., and Matsumoto, K.: Structural basis of the inhibitory effects of Hemoglobin $F$ and Hemoglobin $A_{2}$ on the polymerization of Hemoglobin S. Proc. Nat1. Acad. Sci. USA, 76:670, 1979.

128. Ohba, Y.: Unstable hemoglobins. Hemoglobin, 14:353, 1990.

129. Cathie, I.A.B.: Apparent idiopathic Heinz body anaemia. Great Ormond St. J., 3:43, 1952.

130. Lange, R.D. and Akeroyd, J.H.: Congenital hemolytic anemia with abnormal pigment metabolism and red cell inclusion bodies: A new clinical syndrome. Blood, 13:950, 1958.

131. Scott, J.L., Haut, A., Cartwright, G.E., and Wintrobe, M.M.: Congenital hemolytic disease associated with red cell inclusion bodies, abnormal pigment metabolism and an electrophoretic hemoglobin abnormality. Blood, 16:1239, 1960.

132. Shibata, S., Iuchi, I., Miyaji, T., Ueda, S., and Takeda, I.: Hemolytic disease associated with the production of abnormal hemoglobin and intraerythrocytic Heinz bodies. Acta Haematol. Jap., 26:164, 1963.

133. Sansone, G. and Pik, C.: Familial haemolytic anaemia with erythrocyte inclusion bodies, bilifuscinuria and abnormal haemoglobin (Haemoglobin Galliera Genova). Br. J. Haemato1., 11:511, 1965.

134. Muller, C.J. and Kingma, S.: Haemoglobin Zurich a2AB2 $63 \mathrm{Arg}$. Biochim. Biophys. Acta, 50:595, 1961.

135. Steadman, J.H., Yates, A., and Huehns, E.R.: Idiopathic Heinz body anaemia: Hb-Bristol (B67 (E11) Val->Asp). Br. J. Haematol., 18:435, 1970.

136. White, J.M., Brain, M.C., Lorkin, P.A., Lehmann, H., and Smith, M.: Mild "unstable haemoglobin haemolytic anaemia" caused by Haemoglobin Shepherds Bush (B 74 (E18) Gly->Asp). Nature, 225:939, 1970.

137. Kutlar, F., Felice, A.E., Grech, J.L., Bannister, W.H., Kutlar, A., Wilson, J.B., Webber, B.B., Hu, H., and Huisman, T.H.J.: The linkage

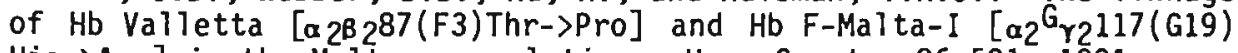
His->Arg] in the Maltese population. Hum. Genet., 86:591, 1991. 
138. Rieder, R.F., Oski, F.A., and Clegg, J.B.: Hemoglobin Philly ( 335 Tyrosine->Phenylalanine): Studies in the molecular pathology of hemoglobin. J. Clin. Invest., 48:1642, 1969.

139. Bunn, H.F., Forget, B.G., and Ranney, H.M.: Unstable hemoglobin variants - Congenital Heinz body hemolytic anemia. In Human Hemoglobins, page 282, W.B. Saunders Company, Philadelphia, 1977.

140. Charache, S., Weatheral1, D.J., and Clegg, J.B.: Polycythemia associated with a hemoglobinopathy. J. C1 in. Invest., 45:813, 1966.

141. Gau, G.T., Fairbanks, V.F., Maldonado, J.F., Bassingthwaighte, J.B., and Tancredi, R.G.: Cardiac dysfunction in a patient with Hemoglobin Malmo treated with repeated transfusions. Clin. Res., 22:276A, 1974.

142. Reed, C.S., Hampson, R., Gordon, S., Jones, R.T., Novy, M.J., Brimha11, B., Edwards, M.J., and Koler, R.D.: Erythrocytosis secondary to increased oxygen affinity of a mutant hemoglobin, Hemoglobin Kempsey. Blood, 31:623, 1968.

143. Bunn, H.F., Wohi, R.C., Bradley, T.B., Cooley, M., and Gibson, Q.H.: Functional properties of Hemoglobin Kempsey. J. Biol. Chem., 249: $7402,1974$.

144. Perutz, M.F., del Pulsinelli, P., Ten Eyck, L., Kilmartin, J.V., Shibata, S., Iuchi, I., Miyaji, T., and Hamilton, H.B.: Haemoglobin Hiroshima and the mechanism of the alkaline Bohr effect. Nature New Biol., 232:147, 1971 .

145. Ikkala, E., Koskela, J, Pikkarainen, P., Rahiala, E-L., El-Hazmi, M.A.F., Nagal, K., Lang, A., and Lehmann, H.: Hb Helsinki: A variant with a high oxygen affinity and a substitution at a 2,3-DPG binding site (B82 [EF6] Lys->Met). Acta Haematol., 56:257, 1976.

146. Jensen, M., Oski, F.A., Nathan, D.G., and Bunn, H.F.: Hemoglobin Syracuse ( $\alpha 2 \beta 2143$ (H21) His->Pro), a new high-affinity variant detected by special electrophoretic methods. J. Clin. Invest., $55: 469,1975$.

147. Horlein, H. and Weber, G.: Uber chronische familiare methamoglobinanue und eine neue modifikation des Methamoglobines. Deutsche Med. Wochenschr., 73:476, 1948.

148. Gerald, P.S. and Efron, M.L.: Chemical studies of several varieties of Hb M. Proc. Nat1. Acad. Sci. USA, 47:1758, 1961.

149. Miyaji, T., Iuchi, I., Shibata, S., Takeda, I., and Tamura, A.: Possible amino acid substitution in the $\alpha$-chain ( $\alpha 87 \mathrm{Tyr}$ ) of Hb M Iwate. Acta Haematol. Jap., 26:538, 1963.

150. Heller, P., Coleman, R.D., and Yakulis, V.: Hemoglobin M Hyde Park: A new variant of abnormal methemoglobin. J. Clin. Invest., 45:1021, 1966.

151. Perutz, M.F., Pulsinelli, P.D., and Ranney, H.M.: Structure and subunit interaction of Haemoglobin M Milwaukee. Nature New Biol., $237: 259,1972$.

152. Hayashi, A., Fujita, T., Fujimura, M., and Titani, K.: A new abnormal fetal hemoglobin, Hb F M-Osaka (a $2{ }_{2} 63$ His->Tyr). Hemoglobin, $4: 447,1980$.

153. Glader, B.E.: Hemoglobin FM-Fort Ripley: Another lesson from the neonate. Pediatrics, 83:792, 1989.

154. Reismann, K.R., Ruth, W.E., and Nomura, T.: A human hemoglobin with lowered oxygen affinity and impaired heme-heme interactions. J. Clin. Invest., 40:1826, 1961. 
155. Bonaventura, J. and Riggs, A.: Hemoglobin Kansas, a human hemoglobin with a neutral amino acid substitution and an abnormal oxygen equi1ibrium. J. Biol. Chem., 243:9880, 1968.

156. Nage 1, R.L., Joshua, L., Johnson, J., Landau, L., Bookchin, R.M., and Harris, M.B.: Hemoglobin Beth Israel: A mutant causing clinically apparent cyanosis. N. Engl. J. Med., 295:125, 1976.

157. Arous, N., Braconnier, F., Thillet, J., Blouquit, Y., Galacteros, F., Chevrier, M., Bordahandy, C., and Rosa, J.: Hemoglobin Saint Mande B102 (G4) Asn->Tyr: A new low oxygen affinity variant. FEBS Lett., $126: 114,1981$. 
10790.

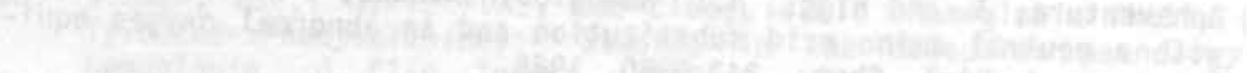

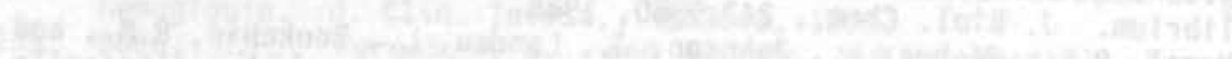

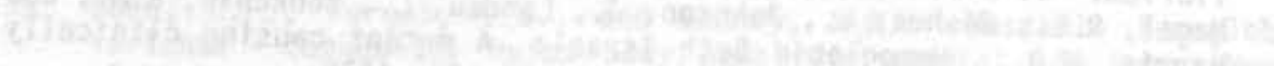

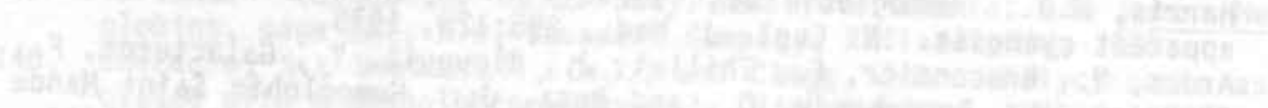

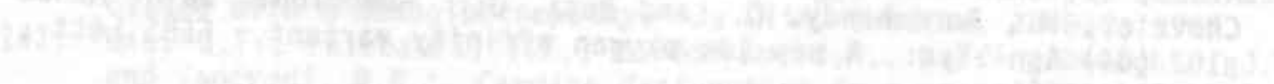

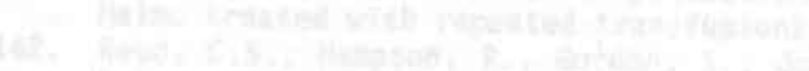

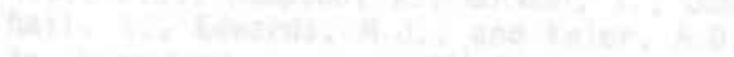

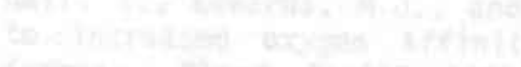

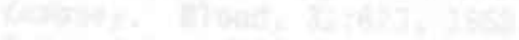

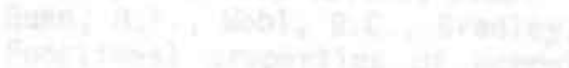

7 $x^{2}$

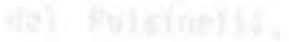

$3^{2}+x^{2}$ 
ADDENDUM

Alphabetical Hb Variant List (Adapted from: Hemoglobin, 17:179, 1993)

Variant Name

$\mathrm{A}_{2}$-Adria

A2-Babinga

$\mathrm{A}_{2}$-Canada

$\mathrm{A}_{2}$-Coburg

$\mathrm{A}_{2}-\mathrm{Fitzroy}$

$\mathrm{A}_{2}-\mathrm{Flatbush}$

$A_{2}$-Grovetown

$\mathrm{A}_{2}$-Honai

$\mathrm{A}_{2}$-Indonesia

$\mathrm{A}_{2}$-Manzanares

$A_{2}$-Melbourne

$\mathrm{A}_{2}$-Nigata

$\mathrm{A}_{2}$-NYU

$A_{2}$-Parkville

$\mathrm{A}_{2}$-Pelendri

$\mathrm{A}_{2}$-Roosevelt

$\mathrm{A}_{2}$-Sphakia

$\mathrm{A}_{2}$-Troodos

$\mathrm{A}_{2}$-Victoria

$\mathrm{A}_{2}$-Wrens

$\mathrm{A}_{2}$-Yialousa

$\mathrm{A}_{2}$-Yokoshima

$\mathrm{A}_{2}$-Zagreb

$\mathrm{A}_{2}{ }^{\prime}\left(\mathrm{B}_{2}\right)$

Aalborg

Abraham Lincoln

Abruzzo

Adana

Agenogi

Aichi

Aida

Al abama

Al-Ain Abu Dhabi

Alamo

Albany-Suma

Alberta

Altdorf

Anantharaj

Andrew-Minneapol is

Ankara

Ann Arbor

Arlington Park

Arya

Residue/Chain/Mutation

Abnormal Property

51 delta Pro->Arg

136 delta Gly->Asp

99 delta Asp->Asn

116 delta Arg->His

142 delta Ala->Asp

22 delta Ala $>$ Glu

75 delta Leu->Val

90 delta Glu->Val

69 delta Gly->Arg

121 delta Glu->Val

43 delta Glu->Lys

1 delta Val->Ala

12 delta Asn->Lys

47 delta Asp->Val

141 delta Leu->Pro

20 delta Val->Glu

2 delta His->Arg

116 delta Arg->Cys

24 delta Giy->Asp

98 delta Val->Met

27 delta Ala->Ser

25 delta Gly->Asp

125 delta Gln->Glu

16 delta Gly->Arg

74 beta Gly->Arg

32 beta Leu->Pro

143 beta His->Arg

59 alpha Gly->Asp

90 beta Glu->Lys

50 alpha His->Arg

64 alpha Asp->Asn

39 beta Gln->Lys

18 alpha Gly->Asp

19 beta Asn->Asp

11 alpha Lys->Asn

101 beta Glu->Gly

135 beta Ala $>$ Pro

11 alpha Lys $\rightarrow$ Glu

144 beta Lys->Asn

10 beta Ala->Asp

80 alpha Leu->Arg

6 beta Glu->Lys;

95 beta Lys $>$ Glu

47 alpha Asp->Asn $\uparrow \mathrm{O}_{2}$ affinity

unstable

thalassemic

thatassemic

unstable

unstable

unstable

unstable

$+\mathrm{O}_{2}$ affinity

unstable

$+\mathrm{O}_{2}$ affinity

sl. unstable

$+\mathrm{O}_{2}$ affinity

unstable; $+\mathrm{O}_{2}$ affinity

$+\mathrm{O}_{2}$ affinity

unstable

(N-Baltimore)

s1. unstable 
Variant Name

Asabara
Atago
Athens-GA
Atlanta
Atlanta-Coventry
Attleboro
Austin
Avicenna
Aztec

Bab-Saadoun

Baden

Barcelona

Bari

Baylor

Beckman

Beijing

Beilinson

Beirut

Belfast

Belliard

Beograd

Beth Israel

Bethesda

Bibba

Bicetre

Birmingham

Bologna

Boras

Bougardirey-Mali

Boumerdes

Boyle Heights

Brest

Brigham

Brisbane

Bristol

British Columbia

Brockton

Bruxelles

Bryn Mawr

Bucuresti

Buenos Aires

Buginese- $X$

Bunbury
Residue/Chain/Mutation

Abnormal Property
74 alpha Asp->His

85 alpha Asp->Tyr

40 beta Arg->Lys

75 beta Leu->Pro

75 beta Leu->Pro;

141 beta Leu->0

138 alpha Ser->Pro

40 beta Arg $\rightarrow$ Ser

47 beta Asp->Ala

76 alpha Met->Thr

48 beta Leu->Pro

18 beta Val->Met

94 beta Asp->His

45 alpha His $>$ Gln

81 beta Leu->Arg

135 beta Ala->Glu

16 alpha Lys $->$ Asn

47 alpha Asp->Gly

126 beta Val->Ala

15 beta Trp->Arg

56 alpha Lys->Asn

121 beta Glu->Val

102 beta Asn->Ser

145 beta $\mathrm{Tyr}->\mathrm{His}$

136 alpha Leu->Pro

63 beta His->Pro

B-141-144 Deleted

(Leu-Ala-His-Lys)->Gln

61 beta Lys->Met

88 beta Leu->Arg

119 beta Gly->Val

37 alpha Pro->Arg

a-6 Deletion Asp->0

127 beta GIn->Lys

100 beta Pro->Leu

68 beta Leu->His

67 beta Val->Asp

101 beta Glu->Lys

138 beta Ala->Pro

B-41 or B-42 Deletion Phe $->0$

85 beta Phe->Ser

42 beta Phe->Leu

85 beta Phe->Ser

116 alpha Glu->Lys

94 beta Asp->Asn
$+\mathrm{O}_{2}$ affinity

unstable

unstable

(Coventry)

$+\mathrm{O}_{2}$ affinity

$+\mathrm{O}_{2}$ affinity; + dissociation

sl. unstable

sl. unstable

unstable; $+0_{2}$ affinity

unstable; $+\mathrm{O}_{2}$ affinity

unstable; $+\mathrm{O}_{2}$ affinity

$+\mathrm{O}_{2}$ affinity

$+\mathrm{O}_{2}$ affinity

unstable; + dissociation

unstable; auto-oxidizing unstable

Inserted

$+\mathrm{O}_{2}$ affinity

unstable

s1. unstable

unstable

unstable

$+\mathrm{O}_{2}$ affinity

unstable

$+\mathrm{O}_{2}$ affinity

unstable

unstable; $+\mathrm{O}_{2}$ affinity

unstable; $+\mathrm{O}_{2}$ affinity

unstable; +02 affinity

unstable; $+\mathrm{O}_{2}$ affinity 
Burke

Bushwick

$\mathrm{B}_{2}\left(\mathrm{~A}_{2}^{\prime}\right)$

C

C-Georgetown

C-Harlem

C-Ziguinchor

Cagliari

Calais

Camden

Camperdown

Caribbean

Casper

Castilla

Catonsville

Chad

Chandigarh

Chapel Hill

Chaya

Chemilly

Chesapeake

Chesterfield

Cheverly

Chiapas

Chiba

Chicago

Chico

Chongqing

Christchurch

City of Hope

Cleveland

Cochin-Port Royal

Cocody

Coimbra

Collingwood

Columbia Missouri

Complutense

Connecticut

Constant Spring

Contaldo
107 beta Gly->Arg

74 beta Gly->Val

16 delta Gly->Arg

6 beta Glu- $>$ Lys

6 beta Glu->Val;

73 beta Asp->Asn

6 beta Glu->Val;

73 beta Asp->Asn

6 beta Glu->Val;

58 beta Pro->Arg

60 beta Val->Gly

76 beta Ala->Pro

131 beta Gln->Glu

104 beta Arg->Ser

91 beta Leu->Arg

106 beta Leu->Pro

32 beta Leu->Arg

a-37 Insertion

$37(+1) 38$

23 alpha Glu->Lys

94 beta Asp->Gly

74 alpha Asp->Gly

43 beta Glu $>$ Gln

99 beta Asp->Val

92 alpha Arg->Leu

28 beta Leu->Arg

45 beta Phe->Ser

114 alpha Pro->Arg

42 beta Phe->Ser

136 alpha Leu->Met

66 beta Lys $>>T h r$

2 alpha Leu->Arg

71 beta Phe->Ser

69 beta Gly->Ser

93 beta Cys->Arg;

121 beta Glu->GIn

146 beta His->Arg

21 beta Asp->Asn

99 beta Asp->Glu

60 beta Val->Ala

88 alpha $A l a->V a l$

127 beta Gln->Glu

21 beta Asp->Gly

$\alpha-141$ Extension

$141(+31)=172$

103 alpha His->Arg
$+\mathrm{O}_{2}$ affinity

unstable

sickling

(Korle Bu)

sickling

(Korle Bu)

sickling

(Dhofar)

unstable

$+\mathrm{O}_{2}$ affinity

s1. unstable

unstable; $+0_{2}$ affinity

unstable; $+0_{2}$ affinity

unstable

unstable; $+\mathrm{O}_{2}$ affinity

$+\mathrm{O}_{2}$ affinity

$+0_{2}$ affinity

unstable; thalassemic

unstable; $+\mathrm{O}_{2}$ affinity

$+\mathrm{O}_{2}$ affinity

unstable; $+\mathrm{O}_{2}$ affinity

unstable

unstable; $+\mathrm{O}_{2}$ affinity

(Okazaki)

$\uparrow \mathrm{O}_{2}$ affinity; polycythemic unstable

$+\mathrm{O}_{2}$ affinity

$+\mathrm{O}_{2}$ affinity

unstable 
Variant Name

Residue/Chain/Mutation

Abnormal Property

\section{Cordele \\ Coventry \\ Cowtown \\ Cranston \\ Crete \\ Creteil}

D-Baltimore
D-Bushman
D-Camperdown
D-Chicago
D-Granada
D-Ibadan
D-Iran
D-Los Angeles
D-Neath
D-North Carolina
D-Ouled Rabah
D-Portugal
D-Punjab
D-St. Louis
D-Washington
Dagestan
Dakar

Dallas

Daneshgah-Tehran

Davenport

Deaconess

Deer Lodge

Denmark Hill

Detroit

Dhofar

Dhonburi

Djelfa

Doha

Drenthe

Duan

Duarte

Duino

Dunn

Durham-N.C.
47 alpha Asp->Ala unstable

B-141 Deletion Leu->0 unstable

146 beta His->Leu $\quad+\mathrm{O}_{2}$ affinity

$\infty-145$ Frameshift unstable; $+\mathrm{O}_{2}$ affinity

$145(+12) \quad->157$

129 beta Ala->Pro

89 beta Ser->Asn

$+\mathrm{O}_{2}$ affinity

68 alpha Asn->Lys

16 beta Gly->Arg

121 beta Glu->Va1

121 beta Glu->Gln

22 beta Glu->Val

87 beta Thr->Lys

22 beta Glu->Gln

121 beta Glu->GIn

121 beta Glu->Ala

121 beta Glu->Gln

19 beta Asn->Lys

121 beta Glu->GIn

121 beta Glu->GIn

68 alpha Asn->Lys

68 alpha Asn->Lys

60 alpha Lys->Glu

v-118 Insertion

$118(+ 3 \longdiv { 1 1 9 }$

97 alpha Asn->Lys

72 aipha His->Arg

78 alpha Asn->His

131 beta Gln->Lys

2 beta His->Arg

95 alpha Pro->Ala

95 beta Lys->Asn

58 beta Pro->Arg

126 beta Val->Gly

98 beta Val->Ala

$$
-1+2+2
$$

$+\mathrm{O}_{2}$ affinity

$+\mathrm{O}_{2}$ affinity

$+\mathrm{O}_{2}$ affinity

$\uparrow \mathrm{O}_{2}$ affinity

$+0_{2}$ affinity

unstable; $+\mathrm{O}_{2}$ affinity

$+\mathrm{O}_{2}$ affinity

unstable

$\uparrow \mathrm{O}_{2}$ affinity

unstable

unstable; $+\mathrm{O}_{2}$ affinity

-1 Extension Met-Glu-His-Leu-

27 beta Ala->Asp unstable

75 alpha Asp->Ala

62 beta Ala->Pro

92 beta His->Pro;

104 beta Arg->Ser

6 alpha Asp->Asn

114 beta Leu->Pro unstable; $+\mathrm{O}_{2}$ affinity unstable

(Newcastle)

unstable; thalassemic 


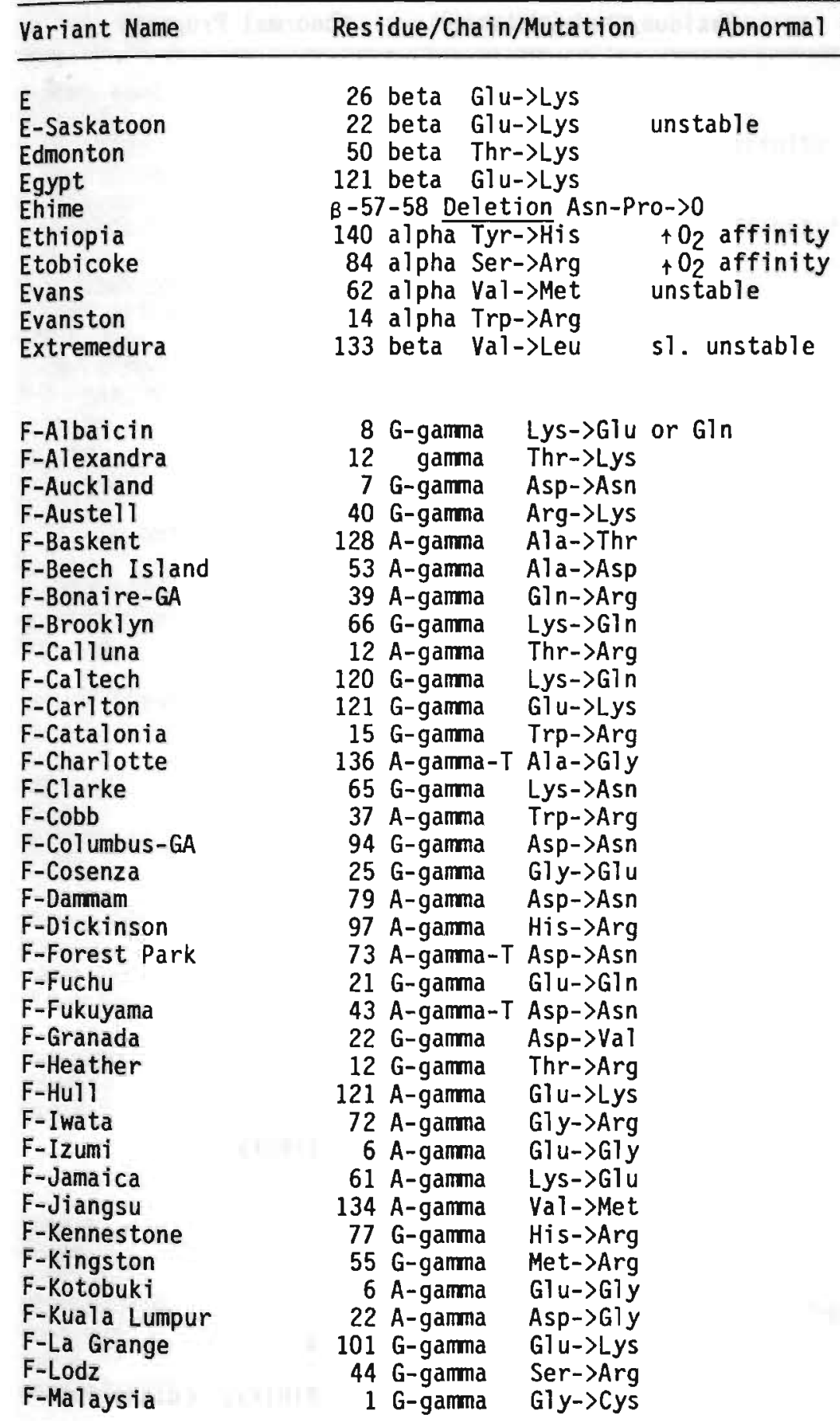


Variant Name

F-Malta-I
F-Marietta
F-Meinohama
F-Melbourne
F-Minoo
F-Oakland
F-Onoda
F-Pendergrass
F-Poole
F-Pordenone
F-Port Royal
F-Sardinia (A T)
F-Shanghai
F-Siena
F-Texas-I
F-Texas-II
F-Tokyo
F-Ube
F-Urumqi
F-Victoria Jubilee
F-Woodstock
F-Xinjiang
F-Xin-Su
F-Yamaguchi
F-M-Fort Ripley
F-M-Osaka

Fannin-Lubbock

Ferndown

Finlandia

Fort de France

Fort Gordon

Fort Worth

Freiburg

Fukuoka

Fukutomi

Fukuyama

G-Accra
G-Audhali
G-Azakouli
G-Bristol
G-Chinese
G-Copenhagen
G-Coushatta
G-Ferrara
G-Gaiveston
G-Georgia

Residue/Chain/Mutation Abnormal Property

\begin{tabular}{|c|c|}
\hline $\begin{aligned} 17 & \text { G-gamma } \\
30 & \text { G-gamma } \\
5 & \text { G-gamma } \\
16 & \text { G-gamma } \\
2 & \text { G-gamma } \\
26 & \text { G-gamma } \\
76 & \text { G-gamma } \\
36 & \text { A-gamma } \\
30 & \text { G-gamma } \\
6 & \text { A-gamma } \\
25 & \text { G-gamma } \\
5 & \text { A-gamma } \\
66 & \text { G-gamma } \\
21 & \text { A-gamma-T } \\
5 & \text { A-gamma } \\
6 & \text { gamma } \\
34 & \text { G-gamma } \\
38 & \text { gamma } \\
22 & \text { G-gamma } \\
30 & \text { A-gamma } \\
40 & \text { A-gamma } \\
25 & \text { A-gamma-T } \\
73 & \text { A-gamma } \\
30 & \text { A-gamma-T } \\
92 & \text { G-gamma } \\
63 & \text { G-gamma }\end{aligned}$ & $\begin{array}{l}\text { His->Arg } \\
\text { Asp->Asn } \\
\text { Glu->Gly } \\
\text { Gly->Arg } \\
\text { Gly->Arg } \\
\text { Glu->Lys } \\
\text { His }>\text { Tyr }+\mathrm{O}_{2} \text { affinity } \\
\text { Pro->Arg } \\
\text { Trp->Gly unstable } \\
\text { Glu->Gln } \\
\text { Glu->ATa } \\
\text { Ile- }>\text { Thr } \\
\text { Lys }>\text { Arg } \\
\text { Glu->Lys } \\
\text { Glu->Lys } \\
\text { Glu->Lys } \\
\text { Val }->\text { Ile } \\
\text { Asn->Lys } \\
\text { Asp->Gly } \\
\text { Asp->Tyr } \\
\text { Arg->Lys } \\
\text { Gly->Arg unstable } \\
\text { Asp->His } \\
\text { Asp->Asn } \\
\text { His }>\text { Tyr cyanosis } \\
\text { His }>>\text { Tyr metHb }\end{array}$ \\
\hline
\end{tabular}

119 beta Gly->Asp sl. unstable

6 alpha Asp->Val $\quad \mathrm{O}_{2}$ affinity

36 beta Pro->Thr $+0_{2}$ affinity

45 alpha His->Arg $\quad+0_{2}$ affinity

145 beta Tyr->Asp $\quad \uparrow 0_{2}$ affinity

27 alpha Glu->Gly

$B-23$ Deletion Val $>0 \quad+0_{2}$ affinity

2 beta His->Tyr

126 alpha Asp->Val

77 beta His->Tyr

73 beta Asp->Asn

23 alpha Glu->Val

68 alpha Asn->Lys

68 alpha Asn->Lys

30 alpha $\mathrm{Gl} u->$ Gln

47 beta Asp->Asn

22 beta Glu->Ala

57 beta Asn->Lys

43 beta Glu->Ala

95 alpha Pro->Leu $\downarrow_{2}$ affinity

unstable

$\mathrm{AO}_{2}$ affinity; + dissociatior 
Variant Name

G-Hong Kong

G-Honolulu

G-Hsi-Tsou

G-Knoxvil1e-I

G-Makassar

G-Norfolk

G-Pest

G-Philadelphia

G-Port Arthur

G-San Jose

G-Saskatoon

G-Singapore

G-Szuhu

G-Taegu

G-Taichung

G-Taipei

G-Taiwan Ami

G-Texas

G-Waimanalo

Gainesville

Galicia

Garden State

Gave110

Geelong

Genova

Gifu

Gothenburg

Grady

Grange Blanche

Graz

Great Lakes

Grenoble

Guangzhou-Hangzhou

Guizhou

Gun Hill

Hafnia

Hamadan

Hamil ton

Hammersmith

Hanamaki

Handa

Handsworth
Residue/Chain/Mutation Abnormal Property

30 alpha Glu->Gln

30 alpha Glu->Gln

79 beta Asp->Gly

68 alpha Asn->Lys

6 beta Glu->Ala

85 alpha Asp->Asn

74 alpha Asp->Asn

68 alpha Asn->Lys

43 beta Glu->Ala

7 beta Glu->Gly

22 beta Glu->Ala

30 alpha GIU->Gln

80 beta Asn $->$ Lys

22 beta Glu->Ala

74 alpha Asp->His

22 beta Glu->Gly

25 beta Gly->Arg

43 beta Glu->Ala

64 alpha Asp->Asn

46 beta Gly->Arg

B-97-98 Deleted unstable

(His-Val)->Leu Inserted

82 alpha Ala->Asp

47 beta Asp->Gly

139 beta Asn->Asp

28 beta Leu->Pro

80 beta Asn $\rightarrow$ Lys

58 alpha His->Tyr

$\alpha-118$ Insertion

$118(+ 3 \longdiv { 1 1 9 }$

27 beta Ala->Val

2 beta His->Leu

68 beta Leu->His

51 beta Pro->Ser;

52 beta Asp->Asn

64 alpha Asp->Gly

77 alpha Pro->Arg

B-91-95 Deletion

Leu-His-Cys-Asp-Lys $>0$

$+\mathrm{O}_{2}$ affinity

$+\mathrm{O}_{2}$ affinity?

unstable
unstable; $\uparrow_{2}$ affinity

$+0_{2}$ affinity

unstable; $\mathrm{t}_{2}$ affinity

$\mathrm{tO}_{2}$ affinity

$+\mathrm{O}_{2}$ affinity

(Osu Christiansborg)

unstable; $\mathrm{O}_{2}$ affinity;

+ dissociation

115 beta His->Gln

56 beta Gly->Arg

11 beta $\mathrm{Val}->$ Ile

42 beta Phe->Ser

139 alpha Lys->Glu

90 alpha Lys->Met

18 alpha Gly->Arg unstable; $t_{2}$ affinity

$\mathrm{O}_{2}$ affinity 


\begin{tabular}{|c|c|c|c|}
\hline Variant Name & Residue/Ch & hain/Mutation & Abnormal Property \\
\hline $\begin{array}{l}\text { Harbin } \\
\text { Hasharon } \\
\text { Hazebrouck } \\
\text { Heathrow } \\
\text { Hekinan } \\
\text { Helsinki } \\
\text { Henri Mondor } \\
\text { Hijiyama } \\
\text { Hikari } \\
\text { Hikoshima } \\
\text { Himeji } \\
\text { Hirosaki } \\
\text { Hirose } \\
\text { Hiroshima } \\
\text { Hobart } \\
\text { Hofu } \\
\text { Hope } \\
\text { Hopkins-I } \\
\text { Hopkins-II } \\
\text { Hörlein-Weber } \\
\text { Hoshida } \\
\text { Hotel-Dieu } \\
\text { Hsin Chu } \\
\text { Hyogo }\end{array}$ & $\begin{array}{r}16 \text { alpha } \\
47 \text { alpha } \\
38 \text { beta } \\
103 \text { beta } \\
27 \text { alpha } \\
82 \text { beta } \\
26 \text { beta } \\
120 \text { beta } \\
61 \text { beta } \\
54 \text { alpha } \\
140 \text { beta } \\
43 \text { alpha } \\
37 \text { beta } \\
146 \text { beta } \\
20 \text { alpha } \\
126 \text { beta } \\
136 \text { beta } \\
95 \text { beta } \\
112 \text { alpha } \\
63 \text { beta } \\
43 \text { beta } \\
99 \text { beta } \\
22 \text { beta } \\
28 \text { beta }\end{array}$ & $\begin{array}{l}\text { Lys->Met } \\
\text { Asp->His } \\
\text { Thr->Pro } \\
\text { Phe->Leu } \\
\text { Glu->Asp } \\
\text { Lys->Met } \\
\text { Glu->Val } \\
\text { Lys }>\text { Glu } \\
\text { Lys->Asn } \\
\text { Gln->Arg } \\
\text { Ala->Asp } \\
\text { Phe->Leu } \\
\text { Trp->Ser } \\
\text { His->Asp } \\
\text { His->Arg } \\
\text { Val->Glu } \\
\text { Gly->Asp } \\
\text { Lys->Glu } \\
\text { His }>>\text { Asp } \\
\text { His }->\text { Tyr } \\
\text { Glu->Gln } \\
\text { Asp->Gly } \\
\text { Glu->Ala } \\
\text { Leu->Pro }\end{array}$ & $\begin{array}{l}\text { unstable; }_{2} \text { affinity } \\
\uparrow_{2} \text { affinity } \\
\mathrm{O}_{2} \text { affinity } \\
\text { unstable } \\
\text { unstable; } \uparrow^{0} \text { affinity } \\
\text { ferriHb; } \mathrm{O}_{2} \text { affinity } \\
\uparrow \mathrm{O}_{2} \text { affinity } \\
\text { unstable; } \uparrow_{2} \text { affinity }\end{array}$ \\
\hline $\begin{array}{l}\text { I } \\
\text { I-Burlington } \\
\text { I-High Wycombe } \\
\text { I-Interlaken } \\
\text { I-Philadelphia } \\
\text { I-Skamania } \\
\text { I-Texas } \\
\text { I-Toulouse } \\
\text { Icaria }\end{array}$ & $\begin{array}{l}16 \text { alpha } \\
16 \text { alpha } \\
59 \text { beta } \\
15 \text { alpha } \\
16 \text { alpha } \\
16 \text { alpha } \\
16 \text { alpha } \\
66 \text { beta } \\
\alpha-141 \text { Exte } \\
141(+31)=1\end{array}$ & $\begin{array}{l}\text { Lys }->\text { Glu } \\
\text { Lys }>\text { Glu } \\
\text { Lys }->\text { Glu } \\
\text { Gly->Asp } \\
\text { Lys->Glu } \\
\text { Lys }->\text { Glu } \\
\text { Lys }->\text { Glu } \\
\text { Lys }>\text { Glu } \\
\text { ension } \\
172\end{array}$ & unstable; ferriHb \\
\hline $\begin{array}{l}\text { Indianapolis } \\
\text { Ingelheim } \\
\text { Inkster } \\
\text { Iowa } \\
\text { I sehara } \\
\text { Istanbul }\end{array}$ & $\begin{array}{r}112 \text { beta } \\
99 \text { beta } \\
85 \text { alpha } \\
119 \text { beta } \\
92 \text { beta } \\
92 \text { beta }\end{array}$ & $\begin{array}{l}\text { Cys->Arg } \\
\text { Asp->Glu } \\
\text { Asp->Val } \\
\text { Gly->Ala } \\
\text { His->Asn->Asp } \\
\text { His->Gln }\end{array}$ & $\begin{array}{l}\text { unstable } \\
\text { unstable; } \uparrow 0_{2} \text { affinity; } \\
\uparrow \text { dissociation }\end{array}$ \\
\hline Iwata & 87 alpha & His->Arg & \\
\hline $\begin{array}{l}\text { J } \\
\text { J-Abidjan } \\
\text { J-Aljezur }\end{array}$ & $\begin{array}{l}54 \text { alpha } \\
51 \text { alpha } \\
12 \text { alpha }\end{array}$ & $\begin{array}{l}\text { Gln->Glu } \\
\text { Gly->Asp } \\
\text { Ala->Asp }\end{array}$ & \\
\hline
\end{tabular}




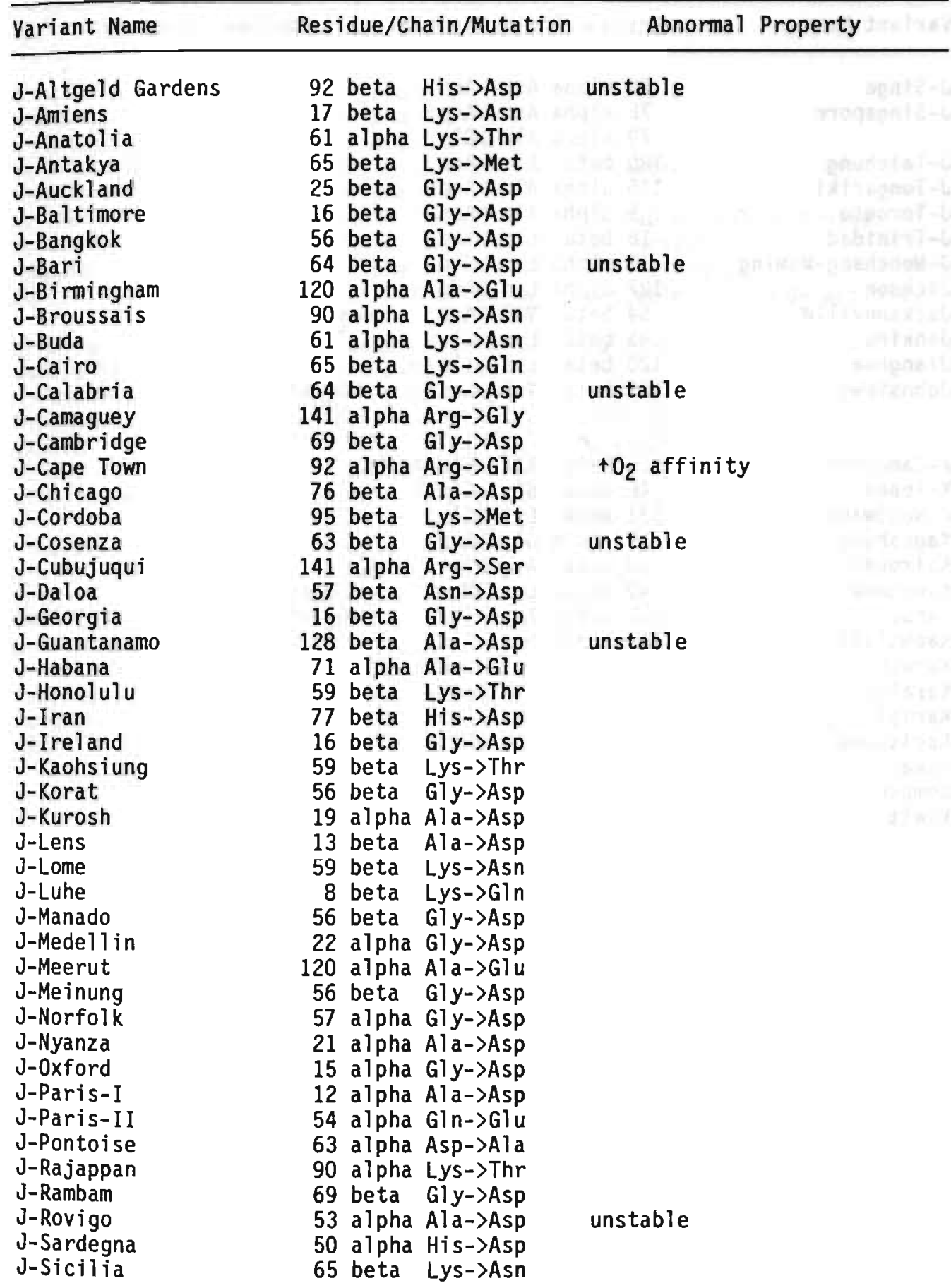


Variant Name

J-Singa

$\mathrm{J}$-Singapore

J-Taichung

J-Tongariki

J-Toronto

J-Trinidad

J-Wenchang-Wuming

Jackson

Jacksonville

Jenkins

Jianghua

Johnstown

K-Cameroon

$\mathrm{K}$-Ibadan

K-Woolwich

Kagoshima

Kairouan

Kanagawa

Kansas

Kaohsiung

Karachi

Karatsu

Kariya

Karlskoga

Kawachi

Kempsey

Kenitra

Kenwood

Kenya

Khartoum

Knossos

Kobe

Kodaira

Kofu

Kokura

Köln

Korea

Kori yama

Korle-Bu

Koya Dora

Kurashiki
Residue/Chain/Mutation

Abnormal Property

78 alpha Asn->Asp

78 alpha Asn->Asp;

79 alpha Ala->Gly

129 beta Ala->Asp

115 alpha Ala->Asp

5 alpha Ala->Asp

16 beta Gly->Asp

11 alpha Lys->Gln

127 alpha Lys->Asn

54 beta Val->Asp

95 beta Lys->Glu

120 beta Lys->Ile

109 beta Val->Leu

unstable

$\uparrow 0_{2}$ affinity

129 beta Ala $>$ Glu or Asp

46 beta Gly->Glu

132 beta Lys->GIn

57 alpha Gly->Asp

30 beta Arg->Thr

40 alpha Lys->Met

102 beta Asn->Thr

113 beta Val->Glu

5 alpha Ala->Pro

120 beta Lys->Asn

40 alpha Lys->Glu

21 beta Asp->His

44 alpha Pro->Arg

99 beta Asp->Asn

69 beta Gly->Arg

$\mathrm{\uparrow}_{2}$ affinity

95 beta Lys $>$ Glu

Fusion $A_{Y}-81 / B-86$

124 beta Pro->Arg

27 beta Ala->Ser

32 beta Leu->Pro

146 beta His->Gin

84 beta Thr->Ile

47 alpha Asp $\rightarrow$ Gly

98 beta Val->Met

B-33-34 Deletion

$\mathrm{O}_{2}$ affinity

$+\mathrm{O}_{2}$ affinity; $\uparrow$ dissociation

B-95 Insertion

$95(+ 5 \longdiv { 9 6 }$

73 beta Asp->Asn

$\alpha-141$ Extension

$141(+1 \overline{6})=157$

74 alpha Asp->His unstable

unstable

$\mathrm{t}_{2}$ affinity

unstable; $\uparrow_{2}$ affinity unstable; $\mathrm{\uparrow}_{2}$ affinity unstable; thalassemic

unstable

$+\mathrm{O}_{2}$ affinity 
Variant Name

L-Ferrara

L-Gaslini

L-Persian Gulf

La Desirade

La Lamentin

La Roche-Sur-Yon

Las Palmas

Legnano

Leiden

Leipzig

Lepore-Baltimore

Lepore-Holl andia

Lepore-Washington-Bosto

Leslie

Lille

Lincoln Park

Linköping

Little Rock

Loire

Long Island-Marseille Louisville

Lufkin

Luxembourg

Lyon

M-Akita

M-Arhus

M-Boston

M-Chicago

M-Emory

M-Erlangen

M-Hida

M-Hyde Park

M-Iwate

M-Kankakee

M-Kiskunhalas

M-Kurume

$\mathrm{M}$-Milwaukee-I

$M-01$ denburg

$\mathrm{M}$-Osaka

M-Radom

M-Saskatoon

M-Sendai
Residue/Chain/Mutation

Abnormal Property

unstable

47 alpha Asp->His

47 alpha Asp->Gly

57 alpha Gly->Arg

129 beta Ala->Va]

20 alpha His->GIn

81 beta Leu->His

49 beta Ser->Phe

141 alpha Arg->Leu

$\beta-6$ or $\beta-7$ Deletion GTu->0

63 beta His->Tyr

Fusion $\delta-50 / \beta-86$

Fusion $\delta-22 / \beta-50$

Fusion $\delta-87 / \beta-116$

131 beta GIn->Lys

74 alpha Asp->Ala

unstable

unstable; $\mathrm{t}_{2}$ affinity

s1. unstable

$\mathrm{O}_{2}$ affinity

unstable; sl. $\uparrow_{2}$ affinity

ferriHb; $\uparrow_{2}$ affinity

$\uparrow \mathrm{O}_{2}$ affinity

$+\mathrm{O}_{2}$ affinity

unstable

Fusion $\delta-22 / \beta-50$;

$\delta-137 \quad$ Val $\rightarrow 0$

36 beta Pro->Thr

143 beta His $>$ Gln $\uparrow_{2}$ affinity

88 alpha $A l a->\operatorname{Ser}+0_{2}$ affinity

$$
-1+1+2
$$

B-1 Extension Met-Val-Pro-Leu-

42 beta Phe->Leu unstable; $\uparrow 0_{2}$ affinity

29 beta Gly->Asp

24 alpha Tyr->His

B-17-18 Deletion Lys-VaT->0

unstable

s1. unstable; $\uparrow_{2}$ affinity

92 beta His->Tyr

63 beta His->Tyr

58 alpha His->Tyr

63 beta His->Tyr

63 beta His->Tyr

63 beta His->Tyr

63 beta His->Tyr

92 beta His $>$ Tyr

87 alpha His->Tyr

87 alpha His->Tyr

58 alpha His->Tyr

63 beta His->Tyr

67 beta Val->Glu

87 alpha His->Tyr

58 alpha His->Tyr

63 beta His->Tyr

63 beta His->Tyr

87 alpha His->Tyr
ferriHb

ferriHb; $\mathrm{t}_{2}$ affinity $\mathrm{O}_{2}$ affinity

ferriHb; $\uparrow_{2}$ affinity

ferriHb; $\mathrm{t}_{2}$ affinity

ferriHb; $\uparrow_{2}$ affinity

ferriHb; $\uparrow_{2} \mathrm{O}_{2}$ affinity

ferrithb

ferriHb; $+\mathrm{O}_{2}$ affinity

ferriHb; $+0_{2}$ affinity

$+\mathrm{O}_{2}$ affinity

ferriHb; $\mathrm{t}_{2}$ affinity

ferriHb; $+0_{2}$ affinity

ferriHb; $+0_{2}$ affinity

$+\mathrm{O}_{2}$ affinity

ferriHb; $\mathrm{t}_{2}$ affinity

ferriHb; $+\mathrm{O}_{2}$ affinity

ferriHb; $\mathrm{t}_{2}$ affinity 


\section{Variant Name}

Machida

Madrid

Mahidol

Malay

Ma 1mó

Mani toba

Maputo

Masuda

Matera

Matsue-0ki

McKees Rock5

Meilahti

Memphis

Mequon

Mexico

Michigan-I

Michigan-II

Milledgeville

Minneapol is-Laos

Mississippi

Mito

Miyada

Miyano

Miyashiro

Mizuho

Mizunami

Mizushi

Moabit

Mobile

Monroe

Montefiore

Montgomery

Montreal

Moscra

Motown

Mozhaisk

Mugino

Munakata

Muscat

Muskegon

$\mathrm{N}$-Baltimore

$\mathrm{N}$-Cosenza

$\mathrm{N}$-Memphis

$\mathrm{N}$-New Haven
Residue/Chain/Mutation

Abnormal Property

unstable

115 beta Ala->Pro

74 alpha Asp->His

19 beta Asn->Ser

97 beta His->GIn

102 alpha Ser->Arg

47 beta Asp->Tyr

114 beta Leu->Met;

119 beta Gly->Asp

55 beta Met->Lys

75 alpha Asp->Asn

145 beta Tyr->Term

36 beta Pro- $>$ Thr

23 alpha Glu->Gln

41 beta Phe->Tyr

54 alpha GIn->Glu

47 alpha Asp->Gly

47 alpha Asp->Gly

44 alpha Pro->Leu

118 beta Phe->Tyr

44 beta Ser->Cys

144 beta Lys->Glu

Fusion $\beta-12 / \delta-22$

41 alpha $\mathrm{Thr}->\mathrm{Ser}$

23 beta Val->Gly

68 beta Leu->Pro

83 beta Gly->Asp

75 alpha Asp->Gly

86 alpha Leu->Arg

73 beta Asp->Val

30 beta Arg->Thr

126 alpha Asp->Tyr

48 alpha Leu->Arg

8-72-76 Deleted

(Ala-Arg-Cys-GIn) Inserted

$+\mathrm{O}_{2}$ affinity

sl. unstable

sl. unstable

(Fannin-Lubbock)

unstable

$+\uparrow \mathrm{O}_{2}$ affinity

unstable; $+\mathrm{O}_{2}$ affinity

$+\mathrm{O}_{2}$ affinity

$+\mathrm{O}_{2}$ affinity

$\mathrm{H}_{2}$ affinity

unstable; $\uparrow \mathrm{O}_{2}$ affinity

unstable

sl. $+\mathrm{O}_{2}$ affinity

unstable; $+\mathrm{O}_{2}$ affinity

$+\mathrm{O}_{2}$ affinity

$\uparrow \mathrm{O}_{2}$ affinity

unstable

24 beta Gly->Asp unstable; $+0_{2}$ affinity

131 beta Gln->Glu

92 beta His->Arg

47 alpha Asp->Gly

90 alpha Lys->Met

32 beta Leu->Val

83 beta Gly->Arg

unstable; $+\mathrm{O}_{2}$ affinity

$\uparrow \mathrm{O}_{2}$ affinity

s1. unstable
95 beta Lys->Glu

15 alpha Gly->Asp

95 beta Lys->Glu

16 beta Gly->Asp 


Variant Name Residue/Chain/Mutation Abnormal Property

N-Seattie

$\mathrm{N}$-Timone

Nagasaki

Nagoya

Nancy

Natal

Neapolis

Necker Enfants-Malades Newcastle

New Mexico

New York

Nigeria

Nikosia

Nishik-I

Nishik-II

Nishik-III

Niteroi

Noki

North Chicago

North Shore

North Shore-Caracas

Nottingham

Nouakchott

Novi Sad

Nunobiki

$0-$ Arab

0 -Indonesia

O-Padova

Oak Ridge

Ocho Rios

ogi

Ohio

Okaloosa

Okayama

Okazaki

Oleander

01 iviere

01 msted

Olomouc

Olympia

Osler

Osu Christiansborg

Ottawa

Owari

Ozieri
61 beta Lys->G1u

8 beta Lys $>$ Glu

17 beta Lys->Glu

97 beta His->Pro

145 beta Tyr->Asp

$\alpha$-140-141 Deletion Tyr-Arg->0

126 beta $\mathrm{Va} 1->\mathrm{Gly}$

20 alpha His->Tyr

92 beta His->Pro

100 beta Pro->Arg

113 beta Val->Glu

81 alpha Ser $>$ Cys

17 beta Lys->G1n

57 alpha Gly->Asp

57 alpha Gly->Asp

57 alpha Gly->Asp

B-43-45 Deletion unstable; $\mathrm{\uparrow}_{2}$ affinity

(Phe-Glu-Ser->0) or

unstable; $\mathrm{t}_{2}$ affinity

$\mathrm{H}_{2}$ affinity

$+0_{2}$ affinity

unstable; thalassemic

unstable; $+\mathrm{O}_{2}$ affinity

B -42-44 (Phe-Glu-Ser->0)

76 alpha Met->Lys

36 beta Pro->Ser

134 beta Val->Glu

134 beta Val->Glu

98 beta Val->Gly

114 alpha Pro->Leu

63 beta His->Tyr

141 alpha Arg->Cys

121 beta Glu->Lys

116 alpha Glu->Lys

30 alpha Glu- $>$ Lys

121 beta Glu->Gln

52 alpha Asp->Ala

34 alpha Leu->Arg

142 beta Ala->Asp

48 beta Leu->Arg

2 beta His->GIn

93 beta Cys->Arg

116 alpha Glu->Gln

116 alpha Glu->Lys

141 beta Leu->Arg

86 beta Ala->Asp

20 beta Val->Met

145 beta Tyr->Asp

52 beta Asp->Asn

15 alpha Gly->Arg

121 alpha $\mathrm{Val}$ 1->Met

71 alpha Ala->Val $\uparrow 0_{2}$ affinity

unstable

unstable

unstable; $+\mathrm{O}_{2}$ affinity

$\uparrow$ hydrophobicity

ferriHb; $\uparrow_{2}$ affinity

$+\mathrm{O}_{2}$ affinity

$+0_{2}$ affinity

$+\mathrm{O}_{2}$ affinity

$+\mathrm{O}_{2}$ affinity

unstable; $+\mathrm{O}_{2}$ affinity

$+\mathrm{O}_{2}$ affinity

unstable; $\mathrm{t}_{2}$ affinity

$+\mathrm{O}_{2}$ affinity

$+\mathrm{O}_{2}$ affinity

$+\mathrm{O}_{2}$ affinity 
Variant Name

\section{P-Congo \\ P-Galveston \\ P-Nilotic}

Palmerston North

Parchman

Pasadena

Pavie

Perspolis

Perth

Petah Tikva

Peterborough

Pierre-Benite

Philly

Pitie-Salpetriere

Poissy

Pontoise

Port Huron

Port Phillip

Porto Alegre

Potomac

Prato

Presbyterian

Providence

Pyrgos

Q-India

Q-Iran

Q-Thailand

Quebec-Chori

Queens

Questembert

Quin Hai

Quong Sze

Radcliffe
Rahere
Rainier
Raleigh
Rampa
Rancho Mirage
Randwick
Redondo
Regina
Reims

Residue/Chain/Mutation

Abnormal Property

Fusion $\beta-22 / \delta-116$

117 beta His- $>$ Arg

Fusion $\beta-22 / \delta-50 \quad+0_{2}$ affinity

23 beta Val->Phe $+0_{2}$ affinity

Fusion $\delta-12 / \beta-22 ; \beta-50 / \delta-87$

75 beta Leu- $>$ Arg unstable; $\uparrow 0_{2}$ affinity

135 alpha Val->Glu

64 al pha Asp->Tyr

32 beta Leu->Pro

110 alpha Ala->Asp

111 beta $\mathrm{Val}->$ Phe

90 beta Glu->Asp

35 beta Tyr->Phe

34 beta Val->Phe

56 beta Gly->Arg;

86 beta Ala->Pro

63 alpha $A 1 a->$ Asp

56 alpha Lys->Arg

91 alpha Leu->Pro

9 beta Ser->Cys

101 beta Glu->Asp

31 alpha Arg->Ser

108 beta Asn->Lys

unstable

unstable

unstable; $+\mathrm{O}_{2}$ affinity

$\uparrow \mathrm{O}_{2}$ affinity

unstable; $\uparrow \mathrm{O}_{2}$ affinity

$\uparrow \mathrm{O}_{2}$ affinity

(Hamadan)

unstable

82 beta Lys->Asn->Asp $+\mathrm{O}_{2}$ affinity

83 beta Gly->Asp

64 alpha Asp->His

75 alpha Asp->His

74 alpha Asp->His

87 beta $\mathrm{Thr}->\mathrm{Ile}$

34 alpha Leu->Arg

131 alpha Ser->Pro

78 beta Leu->Arg

125 alpha Leu->Pro

$\uparrow \mathrm{O}_{2}$ affinity

highly unstable unstable

$\uparrow \mathrm{O}_{2}$ affinity; polymerization; + heme-heme

$\mathrm{H}_{2}$ affinity 
Variant Name

Residue/Chain/Mutation

Abnormal Property

Richmond

Rio Grande

Riverdale-Bronx

Riyadh

Roseau-Pointe a Pitre

Rothschild

Rouen

Rush

Russ

$S$

S-Antilles

S-Oman

S-Providence

S-Travis

Sabine

Saint-Jacques

Saint Mande

Saitama

Saki

San Diego

San Francisco (Pacific)

Santa Ana

Sarrebourg

Sassari

Savannah

Savaria

Saverne

Sawara

Seal Rock

Sealy

Seattle

Sendagi

Serbia

Setif

Shaare Zedek

Shanghai

Shelby

Shenyang

Shepherds Bush

Sherwood Forest
102 beta Asn->Lys

8 beta Lys->Thr

24 beta Gly->Arg

120 beta Lys->Asn

90 beta Glu->Gly

37 beta Trp->Arg

140 alpha Tyr->His

101 beta Glu->Gln

51 alpha Gly->Arg

6 beta Glu->Val sickling

6 beta Glu->Val; sickling

23 beta Val->Ile

6 beta Glu->Val; sickling

121 beta Glu->Lys (0-Arab)

6 beta Glu->Val; sickling

82 beta Lys->Asn->Asp (Providence)

6 beta Glu->Val; sickling; $+\mathrm{O}_{2}$ affinity

142 beta Ala->Val

91 beta Leu->Pro unstable

140 beta Ala $\rightarrow$ Thr $\quad \uparrow 0_{2}$ affinity

102 beta Asn->Tyr $\quad+02$ affinity

117 beta His->Pro unstable

14 beta Leu->Pro unstable

109 beta Val->Met $\uparrow_{2}$ affinity

unstable; $\uparrow \mathrm{O}_{2}$ affinity

unstable

unstable

88 beta Leu->Pro

131 beta Gln->Arg

126 alpha Asp->His

24 beta Gly->Val

49 alpha Ser->Arg

B -143 Frameshift

$143(+13) \quad \rightarrow 156$

6 alpha Asp->Ala

$\alpha-141$ Extension

$141(+31)=172$

47 alpha Asp->His

70 beta Ala->Asp

42 beta Phe->Val

112 alpha His $->$ Arg

94 alpha Asp->Tyr

56 alpha Lys->Glu

131 beta Gln->Pro

131 beta Gin->Lys

26 alpha Ala->Glu

74 beta Gly->Asp

104 beta Arg->Thr unstable

$\mathrm{H}_{2}$ affinity

unstable

$+0_{2}$ affinity

unstable; $+\mathrm{O}_{2}$ affinity

unstable; $\downarrow_{2}$ affinity

unstable

unstable

unstable; $\mathrm{tO}_{2}$ affinity unstable; $_{2}$ affinity 
Variant Name

Residue/Chain/Mutation

Abnormal Property

Shimonoseki

Shuangfeng

54 alpha Gln->Arg

27 alpha Glu->Lys

Siam

Sinai

Singapore

Siriraj

Sogn

Southampton

South Florida

South Milwaukee

Spanish Town

St. Antoine

St. Claude

St. Etienne

St. Francis

St. Louis

St. Lukes

Stanleyville-I

Stanleyville-II

Stanmore

Strasbourg

Strumica

Suan-Dok

Summer Hill

Sunnybrook

Sun Prairie

Sunshine Seth

Suresnes

Swan River

Sydney

Syracuse

T-Cambodia

Tacoma

Tagawa-I

Tagawa-II

Tak

Takamatsu

Ta-11

Tampa

Tarrant

Tashikuergan

15 alpha Gly->Arg

47 alpha Asp->His

141 alpha Arg->Pro

7 beta Glu->Lys

14 beta Leu->Pro

106 beta Leu->Pro unstable; $\uparrow 0_{2}$ affinity $-1+1+2$

B-1 Extension Met-Met-His-Leu-

105 beta Leu->Phe

27 alpha Glu->Val

B -74-75 Deletion

Gly-Leu->0

127 alpha Lys->Thr

92 beta His->Gln

121 beta Glu->Gly

28 beta Leu->Gln

95 alpha Pro->Arg

68 alpha Asn->Lys

78 alpha Asn->Lys

111 beta Val->Ala

23 beta Val->Asp

112 alpha His->Arg

109 alpha Leu->Arg

52 beta Asp->His

36 beta Pro->Arg

130 alpha Ala->Pro

94 alpha Asp->His

141 alpha Arg->His

6 alpha Asp->Gly

67 beta Val->Ala

143 beta His->Pro

26 beta Glu->Lys;

121 beta Glu->Gln

30 beta Arg->Ser

90 alpha Lys->Asn

47 alpha Asp->Gly

B-146 Frameshift

$146(+11) \quad->157$

120 beta Lys->Gln

83 beta Gly->Cys

79 beta Asp->Tyr

126 alpha Asp->Asn

19 alpha Ala->Glu
$+\mathrm{O}_{2}$ affinity

unstable

unstable; $\uparrow^{\uparrow} \mathrm{O}_{2}$ affinity;

$\uparrow$ dissociation

unstable; $\mathrm{t}_{2}$ affinity;

ferriHb

$\uparrow$ dissociation

unstable

unstable

unstable

$+\mathrm{O}_{2}$ affinity

unstable

$\uparrow \mathrm{O}_{2}$ affinity

(D)

unstable; + Bohr \& heme-heme

s1. unstable; $\uparrow_{2} \mathrm{O}_{2}$ affinity

$\uparrow \mathrm{O}_{2}$ affinity 
Variant Name

Terre Haute

Thail and

Thionville

Tianshui

Tigraye

Tilburg

Titusville

Tochigi

Tokoname

Tokuchi

Tonosho

Torino

Tottori

Tours

Toyama

Toyake

Tübingen

Tunis

Turriff

Twin Peaks

Ty Gard

Ube-1

Ube-2

Ube-4

Umi

Uppsala

Vaasa

Valletta

Vancouver

Vanderbilt

Vicksburg

Villejuif

Volga

Waco

Warsaw

Warwickshire

Wayne
Residue/Chain/Mutation

Abnormal Property

106 beta Leu->Arg very unstable

56 alpha Lys->Thr $-1+1+2$

$\alpha-1$ Extension Met-Glu-Leu-Ser

39 beta GIn->Arg

79 beta Asp->His

73 beta Asp->Gly

$\mathrm{H}_{2}$ affinity

94 alpha Asp->Asn

B-56-59 Deletion

Gl y-Asn-Pro-Lys->0

139 alpha Lys->Thr

131 beta Gin->Glu

110 alpha Ala->Thr

43 alpha Phe->Val

59 alpha Gly->Val

$\beta-87$ Deletion

Thr $->0$

136 alpha Leu->Arg

142 beta Ala->Pro

106 beta Leu->Gln

124 beta Pro->Ser

99 alpha Lys->Glu

113 alpha Leu->His

124 beta Pro->Gln

98 beta Val->Met

68 a 7 pha Asn->Asp

116 alpha Glu->Ala

47 alpha Asp->Gly

54 alpha Gln->Glu

39 beta Gln->Glu

87 beta Thr->Pro

73 beta Asp->Tyr

89 beta Ser->Arg

B -75 Deletion Leu->0

123 beta Thr->Ile

27 beta Ala->Asp

40 beta Arg->Lys

42 beta Phe->Val

5 beta Pro->Arg

$\alpha-139$ Frameshift

$139(+ 7 \longdiv { - > 1 4 6 }$
$+\mathrm{O}_{2}$ affinity

$+\mathrm{O}_{2}$ affinity; + dissociation unstable; $\mathrm{O}_{2}$ affinity (?)

$\mathrm{O}_{2}$ affinity

sl. unstable; ${ }^{\uparrow} \mathrm{O}_{2}$ affinity; + dissociation

unstable; $+\mathrm{O}_{2}$ affinity unstable

unstable; $\mathrm{t}_{2}$ affinity

unstable

unstable; $_{2} \mathrm{O}_{2}$ affinity

unstable; $_{2}$ affinity

$+\mathrm{O}_{2}$ affinity

unstable; $\mathrm{t}_{2}$ affinity

$+\mathrm{O}_{2}$ affinity

stable

unstable

${ }^{4} \mathrm{O}_{2}$ affinity

unstable; $+\mathrm{O}_{2}$ affinity

$\uparrow_{2}$ affinity 


\section{Variant Name}

Westmead

Wien

Will lamette

Winnipeg

Wood

Woodville

\section{Yahata}

Yakima

Yamagata

Yatsushiro

Yokohama

York

Yoshizuka

Ypsilanti

Yuda

Yukuhashi

Yukuhashi-II

Yuza

Zaire

Zambia

Zengcheng

Zürich
Residue/Chain/Mutation

Abnormal Property

122 alpha $H$ is->Gln

130 beta Tyr->Asp

51 beta Pro->Arg

75 alpha Asp->Tyr

97 beta His->Leu

6 alpha Asp->Tyr

112 beta Cys->Arg

99 beta Asp->His

132 beta Lys->Asn

60 beta Val->Leu

31 beta Leu->Pro

146 beta His->Pro

108 beta Asn->Asp

99 beta Asp->Tyr

130 alpha Ala->Asp

58 beta Pro->Arg

47 alpha Asp->Gly

21 beta Asp->Tyr

\section{$+\mathrm{O}_{2}$ affinity}

sl. $+\mathrm{O}_{2}$ affinity

unstable

$\uparrow \mathrm{O}_{2}$ affinity

$\downarrow 0_{2}$ affinity

$\uparrow \mathrm{O}_{2}$ affinity

$+\mathrm{O}_{2}$ affinity $\alpha-116$ Insertion

$116(+ 5 \longdiv { 1 1 7 }$

60 alpha Lys->Asn

114 beta Leu->Met

63 beta His->Arg unstable; $\uparrow 0_{2}$ affinity 


\section{CHAPTER 2}

MATERIALS AND METHODS 



\section{CHAPTER 2}

1. Materials and Methods. This chapter provides a description of the methodologies used for the detection and characterization of the $H b$ variants; details of the particular applications are given in Chapters 3 , $4,5,6$, and 7 .

2. Collection of Blood Samples. Several individuals with abnomal $\mathrm{Hb}$ and some of their family members were included in this research project. They originated from different countries (United States, China, India, Canada, and the former Yugoslavia). Some of these patients were detected during newborn screening programs, while others were referred for detailed investigation because of their anemia.

Blood samples were collected in vacutainers with EDTA as anticoagulant (Becton Dickinson Vacutainer System, Rutherford, N.J., USA). The samples were kept cold $\left(4^{\circ} \mathrm{C}\right)$ at all times. Those obtained overseas were transported in wet ice by fast air service to Augusta, GA, USA .

\section{Hematological Evaluation}

3/a Henatological Parameters. Hematological values were obtained with a fully automated cell counter (Sysmex CC-620, Toa Medical Electronics Co., Kobe, Japan). The RBC indices, MCV (in fl) and $\mathrm{MCH}$ (in pg), were calculated from the values of the total $\mathrm{Hb}$ concentration $(\mathrm{g} / \mathrm{dl})$, $\mathrm{PCV}$ (in $1 / 1)$, and RBC counts $\left(x 10^{12 / 1)}\right.$.

3/b Reticulocyte Count. The brilliant cresyl blue stain method was used to estimate the reticulocyte count. Equal parts of stain $(3 \mathrm{~g}$ sodium citrate, $0.9 \mathrm{~g}$ sodium chloride, $1 \mathrm{~g}$ brilliant cresyl blue dissolved in $100 \mathrm{ml}$ distilled water) and blood were mixed in a diluting pipet. The mixture was rotated for 20 minutes, after which a smear was made. Next, the reticulocytes were counted with a microscope.

3/C Peripheral Blood Smears. A moderately thin blood smear was prepared. An air-dried smear was stained with Wright-Giemsa preparation (2 $\mathrm{g}$ Wright's stain, $500 \mathrm{mg}$ Giemsa, $970 \mathrm{ml}$ methanol, and $30 \mathrm{ml}$ glycerol) for 1 minute, and an equal volume of $6.7 \mathrm{mM}$ phosphate buffer was added. After 5 minutes, the slide was washed and air-dried. The cells were examined to evaluate the possible presence of an anisocytosis, poikilocytosis, hypochromia, microcytosis, and of target cells.

3/d Detection of Inclusion Bodies (Heinz bodies). A standard solution of brilliant cresyl blue in physiological saline $(1 \mathrm{~g} / \mathrm{dl})$ was mixed with fresh blood in a ratio of 1:1, and the mixture incubated for 2 hours at $37^{\circ} \mathrm{C}$. A moderately thin blood smear was made and examined under a microscope with an oil immersion lens (100X). Heinz bodies are expected to be present in the blood samples of carriers of an unstable Hb variant (with or without splenectomy). 


\section{Hb Identification and Quantitation}

4/a Preparation of the Hemolysate. Red blood cells were washed three times with isotonic saline and hemolyzed with 1.5 -times volumes of water and 0.4 -times the volume of $\mathrm{CCl}_{4}$ for 15 minutes at room temperature with occasional stirring. When an unstable $\mathrm{Hb}$ was present the addition of $\mathrm{CCl}_{4}$ was avoided. The mixture was then centrifuged at 2,500 $\mathrm{g}$ for 20 minutes and the clear supernatant solution removed with a Pasteur pipet.

4/b IEF. Hb variants were initially detected by IEF using commercially available agarose gels and buffers (Isolab, Inc., Akron, OH, USA). The analysis is sensitive enough to separate $\mathrm{Hb}$ variants with $\mathrm{pIs}$ which differ by 0.02 or greater (1). Usually, $6 \mu 1$ of hemolysate were applied to a gel and electrophoresed for 2 hours at $30 \mathrm{~W}$ constant power using an LKB Multiphor II Horizontal Unit (Pharmacia LKB, Uppsala, Sweden). A pH gradient in the agarose gel is formed during electrophoresis and the $\mathrm{Hb}$ variants migrate through the gel until their individual pis equal the corresponding pH on the gel. Next the gel was fixed in $10 \%$ trichloroacetic acid for 10 minutes, and the bands were visualized with a heme-specific dye (O-dianisidine) and $\mathrm{H}_{2} \mathrm{O}_{2}$. Band identification was by comparing the distances of migration to control containing the known Hb types of $A, F$, $S, C$, and Bart's.

4/c Cation Exchange HPLC. Cation exchange HPLC on a polyCAT A column (4.6 $6200 \mathrm{~mm}$, with a particle size of $5 \mathrm{\mu m}$; PolyLC, Columbia, MD, USA) was used for separation and quantitation of the normal and abnormal Hbs $(2,3)$. The analysis was done on a Waters HPLC system (Waters Chromatography Division, Milford, MA, USA) $(2,3)$. The column was initially purged for 5 minutes with $100 \%$ developer $B$ (35 mM Bis-Tris, $1.5 \mathrm{mM} \mathrm{KCN}, 150 \mathrm{mM}$ Na-acetate, $16.85 \mathrm{mM} \mathrm{NH}_{4}$-acetate, $\mathrm{pH} \mathrm{7.0)}$ and equilibrated for 10 minutes with $25 \%$ developer $B$ and $75 \%$ developer A (35 mM Bis-Tris, $1.5 \mathrm{mM} \mathrm{KCN}, 3 \mathrm{mH}$ $\mathrm{NH}_{4}$-acetate, $\mathrm{pH}$ 6.47). Some 50-200 $\mathrm{gg}$ of $\mathrm{Hb}(10-15 \mathrm{\mu l}$ hemolysate) were applied to the column and the chromatogram was developed with a gradient of 25 to $85 \%$ developer $B$ in 85 minutes with a flow-rate of $0.8 \mathrm{ml} / \mathrm{min}$. The absorbance of the effluent was recorded at $415 \mathrm{~nm}$ with a chart speed of $0.25 \mathrm{~cm} / \mathrm{min}$. Numerous $\mathrm{Hb}$ variants can be separated by this procedure. Fig. $2 / 1$ illustrates the separation of $H b S F, A, A_{2}, S$, and $C$ by this procedure, and gives the positions of the abnormal Hbs studied in this research project.

4/d Reversed Phase HPLC. A large-pore $C_{4}$ column (Vydac, Hesperia, CA, USA) and two water/acetonitrile/TFA developers were used to separate and quantitate the normal and abnormal globin chains $(4,5)$. The separation of globin chains by this method depends primarily on differences in their hydrophobicities. As a general rule, the more polar (less hydrophobic) chains elute earlier in the chromatogram. The presence of TFA in the developers results in a very low $\mathrm{pH}(2.0)$, that causes dissociation of $\mathrm{Hb}$ in heme and globin chains. The column was initially purged with 100\% developer A $(60 \%$ acetonitrile, $0.1 \%$ TFA) and equilibrated for 10 minutes with $50 \%$ developer $A$ and $50 \%$ developer $B$ (20\% acetonitrile, $0.1 \%$ TFA). Some 50-100 $\mathrm{\mu g}$ of $\mathrm{Hb}$ was applied to the column and the chromatogram was developed with a gradient of 50 to $60 \%$ developer $A$ in 60 minutes at a flow-rate of $1.0 \mathrm{ml} / \mathrm{min}$. The absorbance of the effluent was continuously recorded 
at $220 \mathrm{~nm}$ with a chart speed of $0.25 \mathrm{~cm} / \mathrm{min}$. Fig. $2 / 2$ illustrates the separation of globin chains in a newborn sample and gives the positions of the abnormal chains studied.

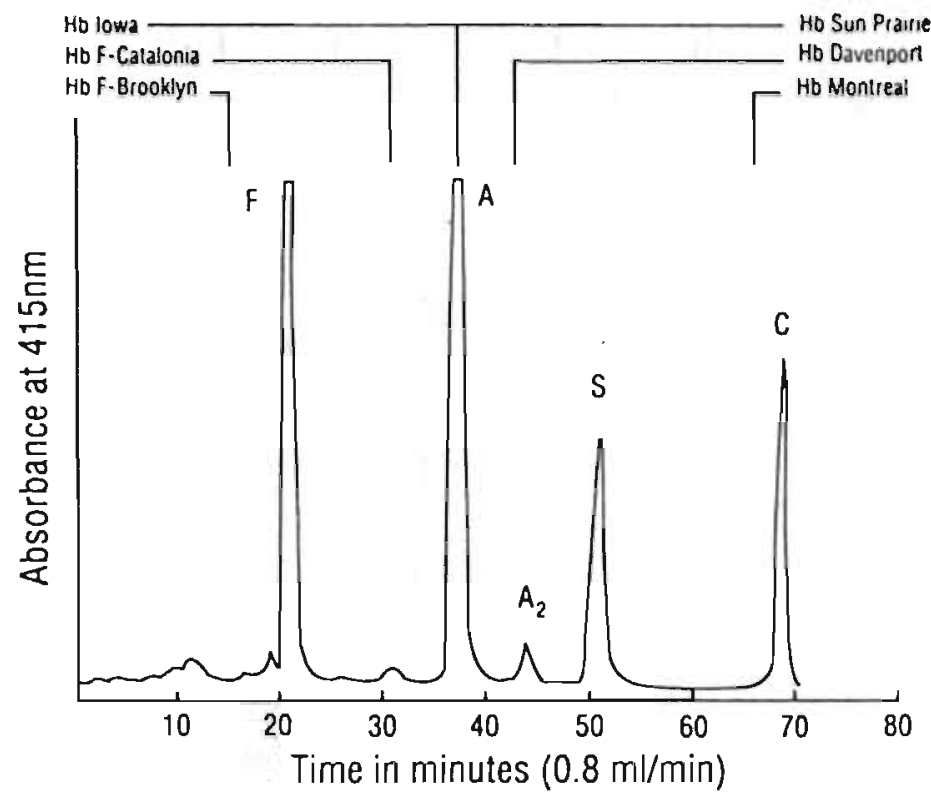

FIG. 2/1. Separation of Hbs $F, A, A_{2} S$, and $C$ in an artificial mixture, by cation exchange HPLC. The positions of the abnormal Hbs detected during this study are also given.

4/e Quantitation of $\mathrm{Hb} A_{2}$ by Microchromatography. In several instances the $\mathrm{Hb} \mathrm{A}$ level was determined by anion exchange DEAE-cellulose chromatography using Tris-HCl developers (6). The resin (DE-52; Whatman, Ltd., Maidstone, Kent, UK) was equilibrated by repeated washings with $0.05 \mathrm{M}$ Tris-HCl, $\mathrm{pH} 8.5,0.1 \% \mathrm{KCN}$. A small column $(0.5-0.8 \times 6 \mathrm{~cm})$ was prepared and about $100 \mu 1$ of diluted hemolysate (1 drop of an $8-12 \mathrm{~g} / \mathrm{dl}$ hemolysate diluted with 6 drops of distilled water and 1 drop of $2 \% \mathrm{KCN}$ ) were slowly applied. After the $H b$ was adsorbed onto the ion exchanger, $0.05 \mathrm{M}$ Tris- $\mathrm{HCl}, \mathrm{pH}$ 8.3, was gently applied. The effluent was collected in a test tube that was calibrated at $5 \mathrm{ml}$. $\mathrm{Hb} \mathrm{A}_{2}$, which was visible on the column as a distinct band about 2 to $5 \mathrm{~mm}$ wide, was eluted in about 30 minutes. The remaining $\mathrm{Hb}$ was eluted with the $0.05 \mathrm{M}$ Tris- $\mathrm{HCl}, \mathrm{pH} 6.5$, and collected in a test tube, calibrated at $20 \mathrm{ml}$. After the volumes of the effluent fractions had been adjusted to 5 and $20 \mathrm{ml}$, respectively, the absorbance values of the two fractions were measured at $415 \mathrm{~nm}$. $H b A_{2}$ was calculated as a $\%$ of the total $\mathrm{Hb}$.

4/f Quantitation of Hb F by Alkali Denaturation. In some instances, Hb $F$ was quantitated by an alkali denaturation procedure that is based on higher resistance of $\mathrm{Hb} F$ to denaturation in alkali (7). The procedure was as follows: 0.2 to $0.3 \mathrm{ml}$ hemolysate containing 5 to $8 \mathrm{~g} / \mathrm{dl} \mathrm{Hb}$ were pipetted into $5 \mathrm{ml}$ of Drabkin's solution ( $1.0 \mathrm{~g}$ sodium bicarbonate, $0.052 \mathrm{~g}$ potassium cyanate, and $0.198 \mathrm{~g}$ potassium ferri-cyanate in $1,000 \mathrm{ml}$ dis- 


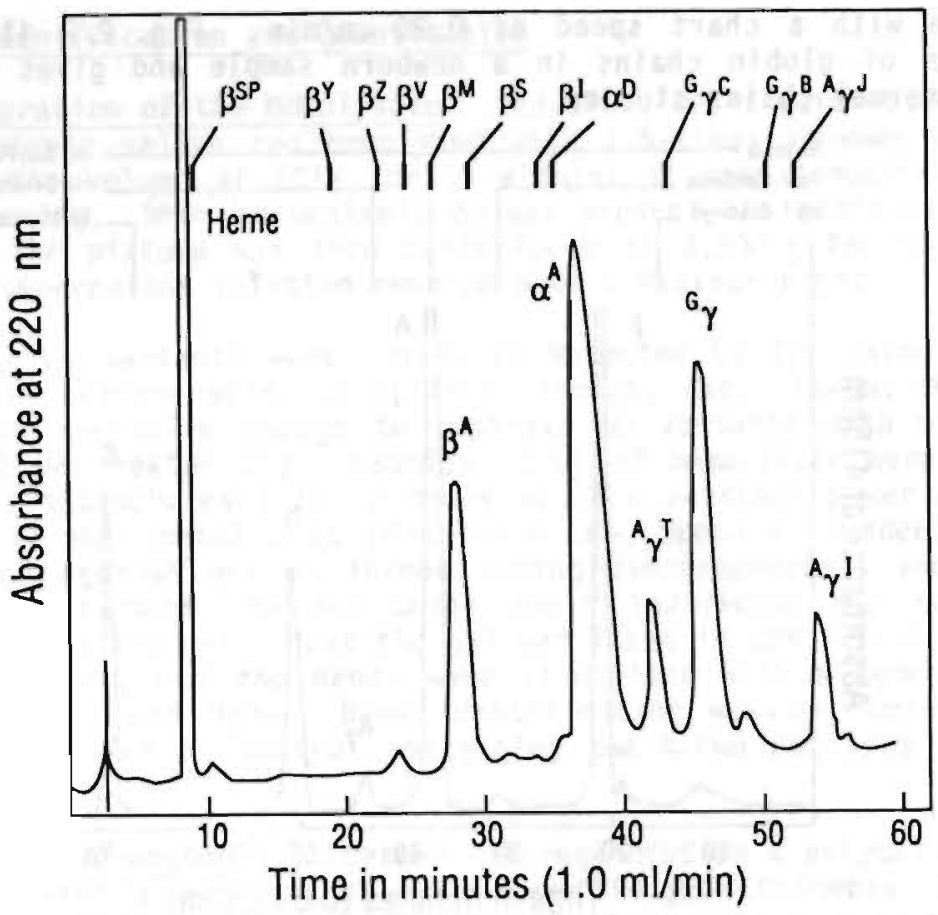

FI6. 2/2. Separation of the common globin chains $\left(B A, \alpha A, A_{\gamma} T, G_{\gamma}\right.$, and $A_{\gamma}$ j) present in a newborn sample by reversed phase HPLC. The positions of the abnormal chains detected during this study are shown. Abbreviations: B, (F-)Brooklyn; C, (F-)Catalonia; D, Davenport; I, Iowa; J, (F-) Jiangsu; M, Montreal; S, S; SP, Sun Prairie; V, Volga; $Y$, Yokohama; $Z$, Zengcheng.

tilled water). The solution was mixed well and $2 \mathrm{ml}$ fractions were transferred into two test tubes (one was used for the test, and one served as a control). At timed intervals, $0.2 \mathrm{ml} 1.2 \mathrm{~N} \mathrm{NaOH}$ were added to the test tube; the solution was carefully mixed, and the denaturing reaction was allowed to proceed for exactly 2 minutes. The reaction was stopped and the denatured $\mathrm{Hb}$ was precipitated by adding $2.0 \mathrm{ml}$ saturated $\left(\mathrm{NH}_{4}\right)_{2} \mathrm{SO}_{4}$. At the same time, $0.2 \mathrm{ml}$ distilled water and $2.0 \mathrm{ml}$ saturated $\left(\mathrm{NH}_{4}\right)_{2} \mathrm{SO}_{4}$ were added to the control tube. Both tubes were allowed to stand for 5 minutes, whereafter the precipitate was removed by filtration through Whatman $\# 1$ filter paper. The $\& \mathrm{Hb} F$ was calculated as the $\% \mathrm{Hb}$ remaining in the solution.

5. Detection of Unstable Hbs. The presence of the unstable Hb variant was demonstrated by the heat stability, isopropanol, and PCMB tests.

5/a Heat Stability Test. In this test the heat stability of the $\mathrm{Hb}$ variant is compared with that of a normal control. The test was performed as follows: $1 \mathrm{ml}$ of a freshly prepared hemolysate containing about $40 \mathrm{mg} \mathrm{Hb}$ was mixed with $40 \mathrm{ml}$ of $0.1 \mathrm{M}$ phosphate buffer, $\mathrm{pH} 7.4$. Five $\mathrm{ml}$ fractions of the diluted $\mathrm{Hb}$ solution was pipetted into eight test tubes. 
The tubes were incubated in a water bath at a constant temperature of $62^{\circ} \mathrm{C}$ for a period of $0,2,4,6,8,10,15$, and 20 minutes. After incubation, the tubes were placed on ice for five minutes and the precipitate removed by centrifugation at $2,500 \mathrm{~g}$ for 10 minutes. The $O D$ 's of the supernatant were determined at $540 \mathrm{~nm}$. The percentage of denatured $\mathrm{Hb}$ in each tube was calculated from the original amount of $\mathrm{Hb}$ present (tube 0 ), and that remaining in solution. A normal control was always simultaneously analyzed and the results obtained for the sample of interest were compared with those of the normal control.

5/b Isopropanol Test. The isopropanol test uses a less polar buffer solution that weakens the hydrophobic bonding of the globin molecule, and allows the test to be done at $37^{\circ} \mathrm{C}(8)$. The test was as follows: $0.2 \mathrm{ml}$ of $10 \%$ hemolysate was transferred to $2 \mathrm{ml}$ of the isopropanol buffer solution (17\% isopropanol in $0.1 \mathrm{M}$ Tris- $\mathrm{HCl}, \mathrm{pH} \mathrm{7.4)}$ contained in a small stoppered tube. The solutions were mixed by inversion and placed in a water bath at $37^{\circ} \mathrm{C}$ for 20 minutes. An unstable $\mathrm{Hb}$ forms a precipitate after 5 minutes and is flocculent after 20 minutes, whereas a normal control hemolysate remains clear.

The preparative isopropanol test was used for the isolation of $\mathrm{Hb}$ Volga (Chapter 5b). Larger volumes were used to allow the isolation of a sufficient quantity of the unstable $\mathrm{Hb}$. The incubation at $37^{\circ} \mathrm{C}$ was stopped at 20 minutes, and the precipitate was isolated by centrifugation. The precipitated $\mathrm{Hb}$ was redissolved in ice-cold $1 \% \mathrm{HCl}$ and its globin was isolated by its addition to ice-cold acetone.

5/c PCMB Test. The PCMB test was first used for the isolation of native $\alpha$ and $\beta$ chains (9). When normal $H b$ reacts with PCMB the protein dissociates into $\alpha$ and $\beta$ subunits, but no precipitation occurs (10). However, an unstable $H b$ not only dissociates more readily when exposed to PCMB, but precipitation of the abnormal chain is also observed. Starch gel electrophoresis is used to evaluate the degree of the dissociation and of the precipitation of the unstable subunit. The technique is useful not so much for screening and detection of unstable Hbs, as for the confirmation and isolation of the abnormal $\alpha$ - and $\beta-g l o b i n$ subunit.

We have noticed that $\mathrm{Hb}$ Yokohama readily precipitates with high concentrations of salt while performing the PCMB test. The procedure used for isolation of $\mathrm{Hb}$ Yokohama is given in Chapter $5 \mathrm{a}$.

\section{Structural Analyses}

6/a Preparative DEAE-Cellulose Chromatography. Preparative anion exchange DEAE-cellulose chromatography with an $\mathrm{NaCl}$ gradient was used for the isolation of $\mathrm{Hb}$ variants that have shown an abnormal electrophoretic behavior. The resin was equilibrated by repeated washings with a stock buffer (0.2 M glycine, $15 \mathrm{mM} \mathrm{KCN}$ buffer, $\mathrm{pH} 7.5)$. A large column (2-3 $\mathrm{x}$ $60-80 \mathrm{~cm}$ ) was prepared and some $200 \mathrm{mg} \mathrm{Hb}$ in the hemolysate were applied. The chromatogram was developed using buffers with varying quantities of $\mathrm{NaCl}$, namely $0.005,0.01,0.02,0.03$, and $0.06 \mathrm{M}$, in the stock buffer. In some instances, the $\mathrm{NaCl}$ gradient was applied to the column by introducing developers with $0.03 \mathrm{M} \mathrm{NaCl}$ from a supply bottle into the closed mixing 
flask containing developer with $0.005 \mathrm{M} \mathrm{NaCl}$. The $\mathrm{Hb}$ fractions were either eluted directly from the column or segments of resin containing the desired $\mathrm{Hb}$ fractions were cut and transferred to a second chromatographic column and eluted with a stripping buffer $(0.01 \% \mathrm{KCN}, 0.2 \mathrm{M} \mathrm{NaCl}, 0.2 \mathrm{M}$ glycine). Large quantities of diluted $\mathrm{Hb}$ solution that were obtained were often concentrated under pressure in an Amicon filtration system (Amicon International Corporation, Danvers, MA, USA) using a Diaflo ultrafiltration membrane PM 10.

6/b CM-Cellulose Chromatography. CM-cellulose chromatography with a sodium phosphate gradient (11) was used to separate a from non-a chains in total hemolysates or in samples of individual Hbs that have previously been isolated by preparative DEAE-cellulose chromatography. CM-cellulose ( $\mathrm{CM}-52)$ was washed three times with deionized water, and 10-20 ml of developer A $\left(0.006 \mathrm{M} \mathrm{Na}_{2} \mathrm{HPO}_{4}, 0.05 \mathrm{M} \mathrm{B}\right.$-mercaptoethanol, $\mathrm{pH} \mathrm{6.5)}$ were added to the resin. The mixture was stirred and poured into a sintered glass column $(1 \times 15 \mathrm{~cm})$ to produce a final bed column of $10 \mathrm{~cm}$ in height. The column was equilibrated with developer $A$ for 1 hour at a flow-rate of $30 \mathrm{ml} /$ hour. About $2 \mathrm{ml}$ of $\mathrm{Hb}$ solution, containing $50-100 \mathrm{mg} \mathrm{Hb}$, was poured with stirring into a suitable centrifuge tube, containing $45 \mathrm{ml}$ of acid acetone (acetone with $1 \% 12 \mathrm{~N} \mathrm{HCl}$ ), previously chilled to $-20^{\circ} \mathrm{C}$. The precipitating globin was recovered by centrifugation and washed once with ice-cold acetone. The globin (40-90 mg) was immediately dissolved in a minimum volume of developer $A$ and applied to the column. Elution was started with developer A for 1 hour, after which a gradient system was introduced that had a linear $\mathrm{Na}^{+}$gradient obtained by mixing $400 \mathrm{ml}$ of developer $A$ with $400 \mathrm{ml}$ of developer $\mathrm{B}\left(0.044 \mathrm{M} \mathrm{Na}_{2} \mathrm{HPO}_{4}, 0.05 \mathrm{M} \mathrm{B}\right.$-mercaptoethanol, pH 6.5), both in open cylinders. Sometimes, in order to improve the separation of some globin chains, different phosphate molarities were used. The effluent was collected with a fraction collector and the protein content of the fractions was analyzed spectrophotometrically at $280 \mathrm{~nm}$. The contents of the tubes, containing an appropriate chain, were collected and urea was removed from the protein solutions by dialysis against water with Spectropor Membrane dialysis tubing having a molecular weight cut-off of approximately 3,500 (Spectrum Medical Industries, Inc., Los Angeles, CA, USA). The protein was recovered by lyophilization.

6/c Preparative Reversed Phase HPLC. Preparative reversed phase HPLC (12) was widely used in this study, especially for the isolation of the abnormal $y$ chains. A preparative $C_{18} \mathrm{HPLC}$ column $(2.15 \times 30 \mathrm{~cm}$, Bio-Sil, TSK-ODS; Bio-Rad Laboratories, Richmond, CA, USA) and two water-acetonitrile-TFA developers were used. The column is capable of loading at least $20 \mathrm{mg} \mathrm{Hb}$. Developer A contained $60 \%$ acetonitrile, $0.1 \%$ TFA, and developer B 20\% acetonitrile, 0.1\% TFA. The column was initially purged with $100 \%$ developer $A$, and equilibrated with the initial percentage of developers from the gradient that has to be applied. Some $5-20 \mathrm{mg} H \mathrm{Hb}$ in hemolysate were applied to the column and the chromatogram was developed with a gradient that allowed the best separation of the abnormal chain in the shortest possible time. Fig. $2 / 3$ illustrates the isolation of the Ay-FJiangsu chain, obtained with a gradient of 62 to $69 \%$ developer $A$ in 135 minutes at a flow-rate of $5 \mathrm{ml} / \mathrm{min}$. The globin chains were detected at a wavelength of $220 \mathrm{~nm}$; the abnormal chain was collected in a beaker and recovered by lyophilization. 


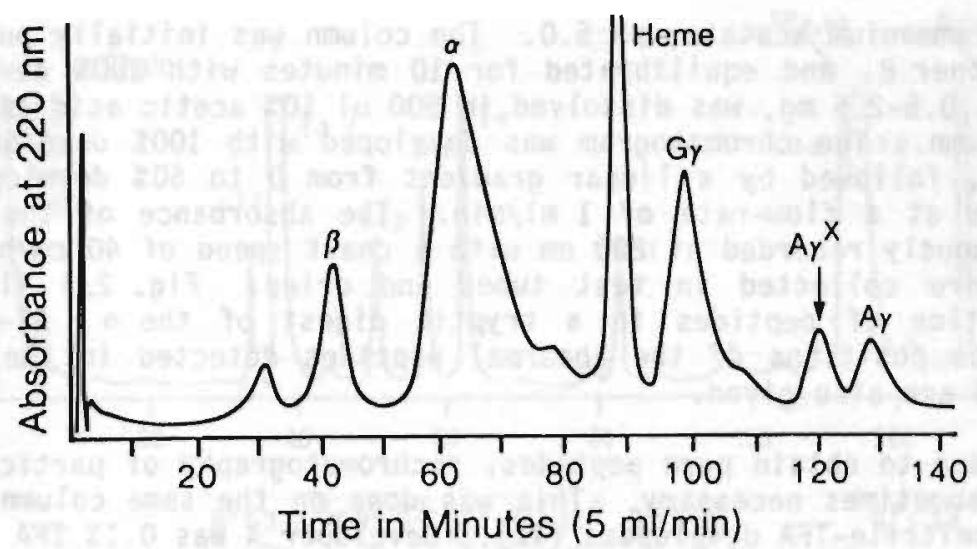

FIG. 2/3. A preparative reversed phase HPL chromatogram on a $\mathrm{C}_{18}$ column. Separation of globin chains in a total hemolysate of a newborn sample with the AY-F-Jiangsu variant.

6/d Aminoethylation of Cysteinyl Residues. The tryptic resistant core peptides were studied after aminoethylation of the cysteinyl residues. The principle of this procedure is that protein sulphydryl groups react with ethylenimine to form $-\mathrm{S}-\mathrm{CH}_{2}-\mathrm{CH}_{2}-\mathrm{NH}_{2}-$ groups. The structure of $\mathrm{AE}-$ cysteine is similar enough to the structure of lysine that peptide bonds involving this derivative will also serve as a substrate for trypsin (13-15). Some $5-10 \mathrm{mg}$ of protein was dissolved in $8 \mathrm{M}$ urea with $0.2 \%$ EDTA and transferred to a $4 \mathrm{ml}$ screw cap vial. Thirty $\mu$ l EDTA solution ( $50 \mathrm{mg}$ EDTA $/ \mathrm{ml}), 300 \mu 1$ Tris-HCl buffer $(5.23 \mathrm{~g} \mathrm{Tris} / 30 \mathrm{ml}, \mathrm{pH} 8.6)$ and $10 \mu 1$ B-mercaptoethanol were added to the protein solution. The vial was completely filled with the $8 \mathrm{M}$ urea and $0.2 \%$ EDTA solution, capped under nitrogen, and kept at room temperature overnight, with constant stirring. Next day, $10 \mu l$ of ethylenimine was added to the solution; the vial was again capped under nitrogen and allowed to remain at room temperature for 2 to 3 hours, with constant stirring. The modified protein was freed of urea and other small molecules by chromatography of the content of the vial through a Sephadex G-25 column in $0.2 \mathrm{~N}$ acetic acid. The proteincontaining fractions were pooled and 1 yophilized.

6/e Tryptic Digestion of Polypeptide Chains. Tryptic digests were made from isolated chains, and in some instances, previously aminoethylated chains. Some 1-5 mg of globin chain was dissolved in 5-10 ml of deionized water. After addition of $4 \mathrm{mg} \mathrm{NH}_{4} \mathrm{HCO}_{3}$, the $\mathrm{pH}$ was adjusted to $8.8-9.0$ with $0.5 \% \mathrm{NaOH}$. Trypsin, dissolved in $0.001 \mathrm{M} \mathrm{HCl}$, was added three times during the digestion, namely at 0,30 , and 120 minutes, in a ratio of $1: 100$ trypsin to protein. The digestion was performed at room temperature, with constant stirring, for 6 hours. The digestion was stopped by lowering the $\mathrm{pH}$ to 2.0 with $1 \mathrm{M} \mathrm{HCl}$. The water was removed by lyophilization.

6/f HPLC Separation of Tryptic Peptides. The peptides, obtained after tryptic digestion, were separated on a large-pore $\mathrm{C}_{18}$ column (Vydac) using a Waters HPLC system (16). The developers used were as follows: Developer A $0.01 \mathrm{M}$ ammonium acetate, $\mathrm{pH}$ 6.0; developer B 50\% acetonitrile, 
$50 \% 0.01 \mathrm{M}$ ammonium acetate, $\mathrm{pH} 6.0$. The column was initially purged with $100 \%$ developer $B$, and equilibrated for 10 minutes with $100 \%$ developer $A$. The sample, $0.5-2.5 \mathrm{mg}$, was dissolved in $500 \mu 1 \mathrm{l} 10 \%$ acetic acid and applied to the column. The chromatogram was developed with $100 \%$ developer $A$ for 10 minutes, followed by a linear gradient from 0 to $60 \%$ developer $B$ for 120 minutes at a flow-rate of $1 \mathrm{ml} / \mathrm{min}$. The absorbance of the effluent was continuously recorded at $220 \mathrm{~nm}$ with a chart speed of $40 \mathrm{~cm} /$ hour. The peptides were collected in test tubes and dried. Fig. 2/4 illustrates the separation of peptides in a tryptic digest of the $\alpha, A E-\beta$, and $\gamma$ chains. The positions of the abnormal peptides detected in the abnormal Hbs studied are also given.

In order to obtain pure peptides, rechromatography of particular peptides was sometimes necessary. This was done on the same column by using water-acetonitrile-TFA developers (17). Developer A was $0.1 \%$ TFA in water, and developer B was $50 \%$ acetonitrile, $0.1 \%$ TFA. The gradient applied for the development of the chromatogram depended upon the retention time in the initial chromatogram of the peptide that has to be purified.

6/g Amino Acid Analyses. The amino acid composition of the isolated tryptic peptides was determined with a Pico-Tag (Waters) amino acid analyzer in the laboratory in Augusta, GA, USA, and with a Beckman 6300 amino acid analyzer (Beckman Instruments, Inc., Palo Alto, CA, USA) in the laboratory in Skopje, Macedonia.

The Pico-Tag method was performed in three steps, according to the procedure recommended of the manufacturer: i) hydrolysis of the peptide samples, ii) pre-column derivatization of the samples, and iii) analysis by reversed phase HPLC.

i. The dried peptide samples were redissolved in $100 \mu 1$ water-acetonitrile solution ( $1: 1$ by volume), and transferred into the sample tubes. The sample tubes were placed into the reaction vial and dried under vacuum on the Work Station. Two hundred $\mu$ l of phenol-HCl solution ( $6 \mathrm{~N} \mathrm{HCl}$ with $1 \%$ phenol) were pipetted onto the bottom of the reaction vial. After three al ternate cycles of vacuum-nitrogen purging on the Work Station, the reaction vial was sealed under vacuum and placed in the oven at $105-110^{\circ} \mathrm{C}$ for 20-24 hours. Following hydrolysis, the samples were evaporated and $10 \mu 1$ of redrying solution (ethanol-water-triethylamine, $2: 2: 1$ by volume) were added to each of the sample tubes. The samples were redried on the Work Station.

ii. Next, the samples were derivatized with PITC to produce PTC amino acids. Twenty $\mu 1$ of freshly prepared derivatization reagent (ethanol-triethylamine-water-PITC, $7: 1: 1: 1$ by volume) were added to each redried sample. The samples were kept at room temperature for 20 minutes, and dried under vacuum on the Work Station.

iii. The PTC amino acids were separated on a reversed phase HPLC $\mathrm{C}_{18}$ column that was kept in a column heater at $38^{\circ} \mathrm{C}$. The developers used were as follows: Developer $A 6 \%$ acetonitrile in $0.14 \mathrm{M}$ sodium acetate trihydrate, $0.05 \%$ TEA, $\mathrm{pH} 6.2$; developer B $60 \%$ acetonitrile, $40 \%$ water. 

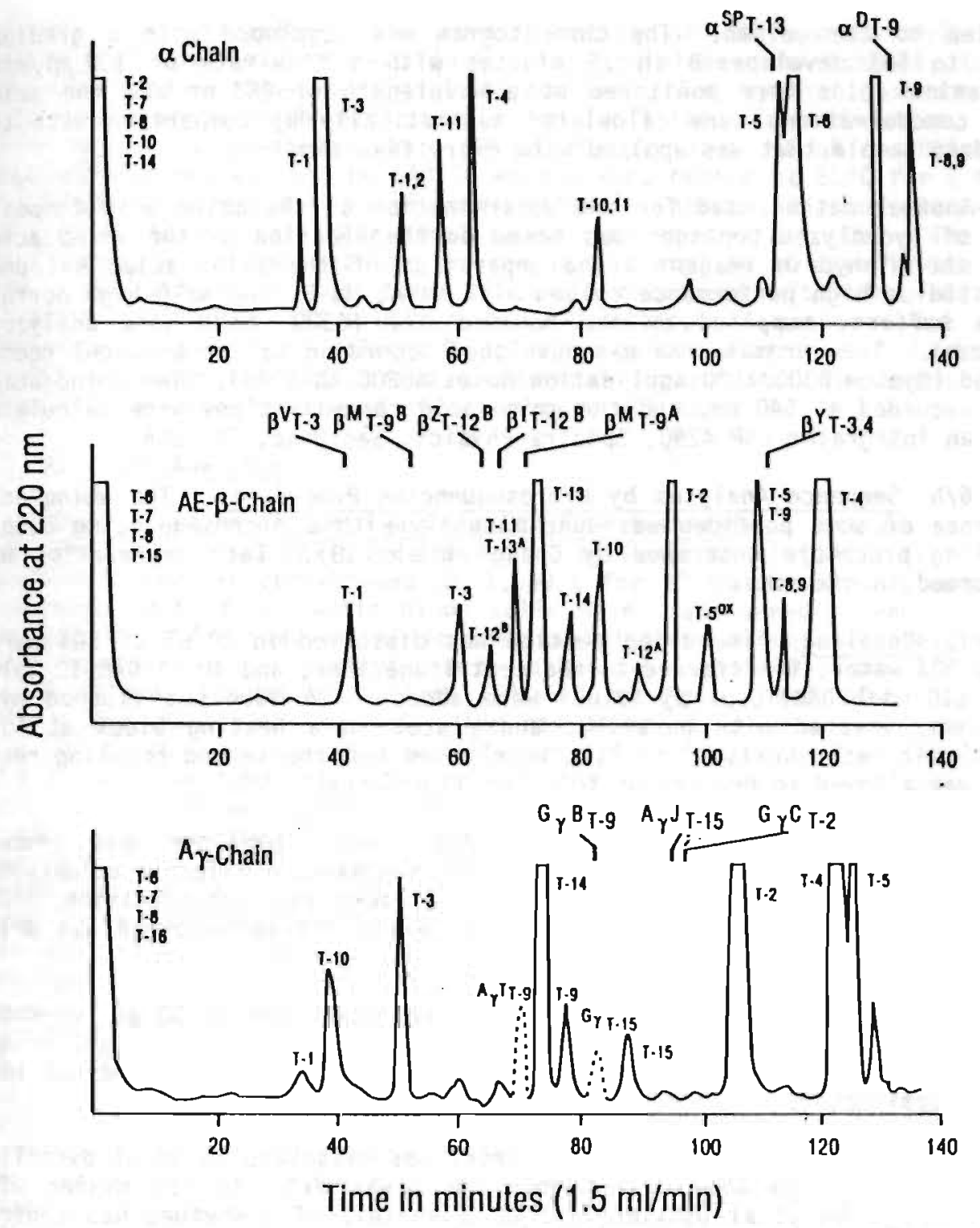

FI6. 2/4. Separation of tryptic peptides by reversed phase HPLC. Shown are soluble tryptic peptides of the $a, A E-\beta$, and $A_{\gamma}$ chains. The positions of peptides $G_{\gamma T-15}$ and $A_{Y T}-9$ are given (peaks with dashed lines). The positions of the abnormal peptides detected in this study are also indicated. Abbreviations: B, (F-)Brooklyn; C, (F-)CataIonia; D, Davenport; I, Iowa; J, (F-) Jiangsu; M, Montreal; S, S; SP, Sun Prairie; V, Volga; $Y$, Yokohama; $Z$, Zengcheng.

The column was initially purged with $100 \%$ developer $B$, and equilibrated with $100 \%$ developer $A$. The samples were dissolved in $40 \mu$ l of sample diluent solution ( $5 \%$ acetonitrile in $5 \mathrm{mM} \mathrm{Na} 2 \mathrm{HPO}_{4}, \mathrm{pH} \mathrm{7.4}$ ) and $20 \mu \mathrm{l}$ were 
applied to the column. The chromatogram was developed with a gradient of 0 to $50 \%$ developer B in 25 minutes with a flow-rate of $1.0 \mathrm{ml} / \mathrm{min}$. The amino acids were monitored at a wavelength of $254 \mathrm{~nm}$ and the amino acid concentrations were calculated automatically by comparison with the standard sample that was applied with every five samples.

Another method used for the determination of the amino acid composition of hydrolyzed peptides was based on the reaction of the amino acids with the ninhydrin reagent. The separation of the amino acids was done on a sodium high performance column with $\mathrm{Na}-\mathrm{A}, \mathrm{Na}-\mathrm{B}$, and $\mathrm{Na}-\mathrm{D}$ high performance buffers, supplied by the manufacturer (6300 amino acid analyzer; Beckman). The chromatogram was developed according to the protocol recommended (System 6300/7300 Application Notes A6300-AN-002B). The amino acids were recorded at $540 \mathrm{~nm}$, and the amino acid concentrations were calculated with an integrator (SP 4290, Spectra Physics, San Jose, CA, USA).

6/h Sequence Analyses by Microsequencing Procedures. The amino acid sequence of some peptides was done manually with a microsequencing double coupling procedure described by Chang et al (18). Each degradation was performed in six steps:

i. Coupling: The dried peptide was dissolved in $80 \mu l$ of $50 \%$ pyridine, $50 \%$ water, transferred to the centrifuge tube, and $40 \mu \mathrm{I}$ DABITC solution (10 nmol DABITC/ $\mu 1$ pyridine) were added. The tube was flushed with nitrogen, covered with parafilm, and placed in a heating block at $50^{\circ} \mathrm{C}$ for 45 minutes. Next, $10 \mu 1$ PITC were added and the second coupling reaction was allowed to proceed at $50^{\circ} \mathrm{C}$ for 30 minutes.

ij. Extraction: The excess reagents and by-products were removed by mixing with two portions of $400 \mu l$ n-heptane:ethylacetate solution (2.5:1 by volume) on a vortex mixer, followed by centrifugation. The organic phase was removed and discarded, while the water phase was dried under vacuum.

iii. Cleavage: The dried material was dissolved in $50 \mu 1$ anhydrous TFA, flushed with nitrogen; the tube was covered with parafilm and heated in a heat block at $50^{\circ} \mathrm{C}$ for 15 minutes. The sample was then dried in a vacuum desiccator.

iv. Extraction: The dried material was dissolved in $30 \mu 1$ distilled water, after which the cleaved amino acid was extracted by mixing with two portions of $50 \mu l$ butylacetate on a vortex. The mixture was centrifuged and the butylacetate extract, that contained the cleaved amino acid, was transferred to a clean tube. The rest of the peptide that remained in the water phase was evaporated and subjected to the next degradation cycle.

v. Conversion: The butylacetate extract was dried and redissolved in $30 \mu 150 \%$ TFA, $50 \%$ water. Conversion of thiazolinones of amino acids into thiohydantoins was carried out at $50^{\circ} \mathrm{C}$ for 50 minutes. The sample was dried and redissolved in 10-20 $\mu$ l ethanol. 
vi. Identification: The identification of DABTH-amino acids was done on micropolyamide sheets $(3 \times 3 \mathrm{~cm}$; Schleicher and Schuell, Keene, $\mathrm{NH}$, USA) with a two-dimensional separation. The synthetic marker was co-chromatographed with each sample to facilitate identification. The marker was prepared as follows: $500 \mu l$ 50\% pyridine, $60 \mu l$ diethylamine, $30 \mathrm{\mu l}$ ethylamine, and $565 \mathrm{\mu g}$ DABITC were mixed, heated at $55^{\circ} \mathrm{C}$ for 1 hour, dried under vacuum and redissolved in ethanol. About 1-2 $\mu 1$ of the marker was applied to the polyamide sheet using a microcapillary tube. The spot was allowed to dry, whereafter the sample was applied at exactly the same spot. The separation was done in small containers with the following solvents: Solvent A $33 \%$ acetic acid, $67 \%$ water, was used for the first dimensional separation; solvent $B$ toluene-n-hexane-acetic acid $(2: 1: 1$ by volume) was used for the second dimensional separation. The sheets were dried and the color developed by exposing to $\mathrm{HCl}$ vapor.

\section{DNA Analyses}

7/a Isolation of Human Genomic DNA From Leukocytes. DNA was isolated from the white blood cells by the method of Poncz et al (19). About 5-10 $\mathrm{ml}$ whole blood was centrifuged at $1,500 \mathrm{~g}$ for 10 minutes; the plasma was discarded; most of the white blood cells were transferred to another tube and used for DNA isolation, while the remaining red blood cells were saved for $\mathrm{Hb}$ analyses. The leukocytes were washed twice with 30-30 ml $1 \mathrm{X}$ reticulocyte saline (104 $\mathrm{mM} \mathrm{NaCl}, 4 \mathrm{mM} \mathrm{KCl}, 6.8 \mathrm{mM} \mathrm{MgCl}$ ) and centrifuged at $2,500 \mathrm{~g}$ for 10 minutes at $4^{\circ} \mathrm{C}$. The red blood cells were hemolyzed with $30 \mathrm{ml}$ of freshly prepared lysing solution $\left[131 \mathrm{mM} \mathrm{NH}_{4} \mathrm{Cl}, 0.9 \mathrm{mH}_{4}\left(\mathrm{NH}_{4}\right)_{2} \mathrm{CO}_{3}\right.$, $\mathrm{pH}$ 6.5] at room temperature for 20 minutes with occasional shaking. The white blood cells were pelleted by centrifugation at $2,500 \mathrm{~g}$ for 10 minutes at $4^{\circ} \mathrm{C}$. The supernatant was removed and the lysing procedure was repeated once more. The pellet, consisting mainly of white blood cells, was resuspended in $10 \mathrm{ml}$ STE buffer $(0.1 \mathrm{M} \mathrm{NaCl}, 0.05 \mathrm{M} \mathrm{Tris-HCl,} \mathrm{pH} \mathrm{7.5,} 1 \mathrm{mM}$ EDTA) and $1 \mathrm{ml}$ of $10 \% \mathrm{SDS}$, whereafter $0.1 \mathrm{ml}$ of proteinase $\mathrm{K}(10 \mathrm{mg} / \mathrm{ml}$ in $10 \mathrm{~m} / \mathrm{M}$ Tris-HC1, $\mathrm{pH} 7.5,1 \mathrm{mM}$ EDTA) was added; the mixture was incubated at $37^{\circ} \mathrm{C}$ overnight, without shaking. The mixture became viscous upon incubation. The proteins were removed by phenol-chloroform-isoamylalcohol extractions as follows: An equal volume of phenol saturated with $20 \mathrm{mM} \mathrm{Tris-HCl,} \mathrm{pH}$ 8.0, containing $0.1 \%$ hydroxyquinoline as an antioxidant, was added to the viscous solution, and the mixture was gently shaken for 10 minutes at room temperature, cooled on ice for 10 minutes, and centrifuged at $2,500 \mathrm{~g}$ for 10 minutes at $4^{\circ} \mathrm{C}$. The upper aqueous phase was transferred to a clean tube and the phenol extraction was repeated once more. Next, an equal volume of chloroform-isoamylalcohol (19:1 by volume) was added; the mixture was gently shaken for 10 minutes and centrifuged at $2,500 \mathrm{~g}$ for 10 minutes at $4^{\circ} \mathrm{C}$. The upper aqueous phase was transferred to a fresh tube and the DNA was precipitated with 5 volumes of ice-cold absolute ethanol. The DNA precipitate was transferred to a clean $1.5 \mathrm{ml}$ Eppendorf tube, washed tivice with $70 \%$ ethanol, and dried under vacuum. The pellet was dissolved in $1 \mathrm{ml}$ TE buffer ( $10 \mathrm{mM}$ Tris-HCl, $\mathrm{pH} 7.5,1 \mathrm{mM} \mathrm{ETA})$ by incubation at $37^{\circ} \mathrm{C}$ overnight. The DNA concentration was determined from its optical density at $260 \mathrm{~nm}(1 \mathrm{OD}=50 \mathrm{\mu g} / \mathrm{ml}$ DNA $)$. The DNA was stored at $4^{\circ} \mathrm{C}$. 
7/b PCR Amplification. PCR is based upon the in vitro amplification of a specific DNA fragment flanked by two synthetic oligonucleotide primers, complementary to the opposite strands of the desired fragment (20). Repeated cycles of denaturation, annealing, and extension result in an exponential accumulation of the target fragment $\left(10^{6}-\right.$ fold $)$. The use of a heat-stable DNA polymerase and a programable thermocycler automates the procedure (21). The PCR method was used in order to obtain large amounts of specific double-stranded DNA fragments, used in the dot-blot hybridization, and for restriction enzyme digestion or to generate large amounts of specific single-stranded DNA fragments, used in DNA sequencing analysis.

The oligonucleotides, serving as amplification primers, as well as the sequencing primers used in the DNA sequencing analysis and specific probes used in the dot-blot analysis, were synthesized by a solid-phase oligoncleotide synthesis method (22) on a 380 B DNA synthesizer (Applied Biosystems, Inc., Foster City, CA, USA) in the laboratory in Augusta, GA, and on a Plus 1 DNA synthesizer (Beckman) in the laboratory in Skopje, Macedonia.

The PCR was performed in three phases using an Automated Thermocycler (Perkin Elmer Cetus, Norwalk, CT, USA). The reaction mixture contained $1 \mu \mathrm{g}$ genomic DNA, $20 \mathrm{mM}$ MOPS, $\mathrm{pH} 7.8,50 \mathrm{mM} \mathrm{NaCl}, 2.5 \mathrm{mM} \mathrm{MgCl} 2,200 \mu \mathrm{M}$ of each dNTP, and $100 \mathrm{pmol}$ of each of the oligonucleotide primers, in a total volume of $100 \mu \mathrm{l}$. For the generation of single-stranded DNA templates, an unbalanced ratio of $100 \mathrm{pmol}$ to 1 pmol was used. In the first phase, the genomic DNA was denatured at $95^{\circ} \mathrm{C}$ for 5 minutes. The temperature was decreased to $80^{\circ} \mathrm{C}$ and $2.5 \mathrm{U}$ of Taq polymerase was added to each sample. Samples were overlaid with 2-3 drops of mineral oil to prevent evaporation. In the second phase, the samples were subjected to 35 repeated cycles of denaturation at $95^{\circ} \mathrm{C}$ for 1 minute, annealing of the primers at $56^{\circ} \mathrm{C}$ for 1 minute, and extension of the annealed primers at $72^{\circ} \mathrm{C}$ for 2 minutes. The temperature of annealing depends upon the sequences of the primers, while the time of the primer extension depends upon the length of the region that has to be amplified. In the third phase, the samples were incubated for 10 minutes at $72^{\circ} \mathrm{C}$, so that a complete extension of all DNA strands synthesized in the previous step was obtained. The amplification product was checked by applying $5 \mu 1$ of each reaction on a $1.5 \%$ agarose gel in the presence of ethidium bromide $(10 \mu \mathrm{g} / \mathrm{ml})$ along with a Hind IIIdigested $\lambda$ DNA as a size marker. The samples were kept at $4^{\circ} \mathrm{C}$ for further analyses.

7/c DNA Sequencing. DNA sequencing was used in this study for the determination of the primary sequence of the coding region of the abnomal B-Montreal and $\beta$-Yokohama genes. The analys is was performed on a singlestranded, PCR-amplified, DNA template in three phases: i) purification of the single-stranded DNA template, ii) sequencing reaction, and iii) denaturing PAGE.

i. Purification of the Single-Stranded DNA Template: The singlestranded amplified DNA was purified by precipitation with 1 volume of $2.5 \mathrm{M}$ ammonium acetate and 2 volumes of ice-cold ethanol at room temperature for 15 minutes. The mixture was centrifuged for 15 minutes at $4^{\circ} \mathrm{C}$; the 
resulting pellet washed four to five times with $70 \%$ ethanol, dried under vacuum, and dissolved in $10 \mu \mathrm{T}$ water. The recovery and DNA concentration were estimated on a $1.5 \%$ agarose gel by comparison with $0.3 \mu \mathrm{g} \mathrm{M}-13$ control single-strand DNA.

ii. Sequencing Reaction: The sequencing reaction was done with a dideoxy chain termination method (23), using a Sequenase Version 2.0 DNA sequencing kit [United States Biochemical (USB), Cleveland, OH, USA]. The reaction was performed in three steps (annealing, labeling, and termination), following the protocol recommended by the manufacturer.

The following reagents were used to anneal the primer to the template: $50 \mu 1$ of sequencing primer, $2 \mu 1$ of $5 X$ reaction buffer $(200 \mathrm{mM} \mathrm{Tris}-\mathrm{HCl}$, $\mathrm{pH} 7.0,100 \mathrm{mM} \mathrm{MgCl} 2,250 \mathrm{mM} \mathrm{NaCl}), 0.3 \mu \mathrm{g}$ of the purified single-stranded DNA template in a total volume of $10 \mu \mathrm{l}$, adjusted with sterile water. The mixture was incubated at $65^{\circ} \mathrm{C}$ for 3 minutes and allowed to cool slowly to room temperature over a period of about 30 minutes.

The labeling reaction was achieved by adding the following reagents to the annealed template: $1.0 \mu 10.1 \mathrm{M}$ DTT, $2.0 \mu 1$ diluted labeling mix (7.5 $\mu \mathrm{M}$ dATP, $7.5 \mu \mathrm{M}$ dTTP), $0.5 \mu 1 \alpha-35 \mathrm{~S}$-dATP $(800 \mathrm{Ci} / \mathrm{mM}, 10.0 \mathrm{mCi} / \mathrm{ml}$; Dupont, Boston, MA, USA), and $2.0 \mu 1$ of diluted sequenase enzyme. The

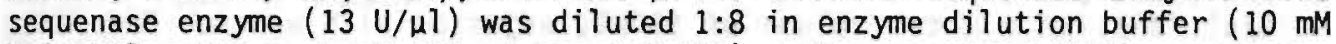
Tris- $\mathrm{HCl}, \mathrm{pH} 7.5,5 \mathrm{mM} \mathrm{DTT}, 0.5 \mathrm{mg} / \mathrm{ml} \mathrm{BSA})$. The reaction mixture was incubated at room temperature for 3 minutes.

Termination reaction: $2.5 \mu 1$ of each termination mixture (ddATP, ddCTP, ddGTP, ddTTP) were pipetted into four tubes labeled $A, C, G$, and T. Each termination mixture consists of $50 \mu \mathrm{M} \mathrm{NaCl}, 80 \mu \mathrm{M}$ of each dATP, dCTP, dGTP, and dTTP, and $8 \mu \mathrm{M}$ of the corresponding ddNTP. The tubes were prewarmed at $37^{\circ} \mathrm{C}$ for 1 minute. After the labeling reaction was completed, $3.5 \mu 1$ of the reaction mixture was added to each of the labeled tubes. The tubes were incubated at $37^{\circ} \mathrm{C}$ for 5 minutes, after which the reaction was stopped by adding $4 \mu 1$ of Stop solution ( $95 \%$ formamide, $20 \mathrm{mM}$ EDTA, $0.05 \%$ Bromphenol Blue, $0.05 \%$ xylenecyanol FF). The samples were kept on ice until loaded onto the gel.

iii. Denaturing Gel Electrophoresis: A $0.4 \mathrm{~mm}$ thick $8 \%$ polyacrylamide gel in $7 \mathrm{M}$ urea-TBE buffer gel was prepared 2 to 20 hours prior to use. The gel was run on a vertical electrophoresis apparatus for DNA sequencing (Model S2; Bethesda Research Laboratories, Gibco-BRL, Gaithersburg, MD, USA) using a high voltage constant power supply (2297-Microdrives; LKB-Broma, Uppsala, Sweden). Prior to loading the samples, the gel was pre-electrophoresed for 30 minutes at $70 \mathrm{~W}$ constant power. The DNA samples were denatured at $75-80^{\circ} \mathrm{C}$ for 3 minutes and $3 \mu 1$ of each reaction were applied to the gel. The gel was run at $75 \mathrm{~W}$ constant power $(1,400-1,600 \mathrm{~V} ; 50-60 \mathrm{~mA})$ for $2-4$ hours. Next, the gel was soaked in a $10 \%$ acetic acid-12\% methanol solution for 30 minutes, transferred to (Whatman) 3 MM paper, and dried under vacuum at $80^{\circ} \mathrm{C}$ on a gel dryer ( $\mathrm{Slab}$ Dryer, Model 483; Bio-Rad) for about 40 minutes. The dried gel was exposed directly to Kodak X-ray film (Eastman Kodak Company, Rochester, N.Y., USA) for 10 to 16 hours. 
7/d Dot-Blot Hybridization Analyses. Dot-blot hybridization was used for the verification of the nt changes found in Hb Montreal and for the localization of the mutation in $\mathrm{Hb}$ Sun Prairie on the a-globin genes. The analyses were performed in three steps: i) preparation of dot-blot membranes, ii) $5^{\prime}$ end-labeling of the normal and mutant oligonucleotides and their purification, and $i i i)$ dot-blot hybridization.

i. Preparation of Dot-Blot Membranes: The double-stranded PCR amplified DNA was denatured by adding $20 \mu l 4 \mathrm{M} \mathrm{NaOH}, 0.25 \mathrm{M}$ EDTA in a total volume of $200 \mu \mathrm{l}$, adjusted with water. The mixture was kept at room temperature for 30 minutes. The denatured DNA was blotted in duplicate onto a nylon membrane (Zeta Probe Nylon Membrane; Bio-Rad) under vacuum using a spotting device (Bio-Dot; Bio-Rad). The DNA was fixed to the membrane by baking in a vacuum oven at $80^{\circ} \mathrm{C}$ for 2 hours.

ii. 5' End-Labeling of the 01 igonucleotides and Their Purification: Two allele-specific oligonucleotide probes (normal and mutant) were labeled at the $5^{\prime}$ end with $\gamma^{32 \mathrm{p}-A T P}(7,000 \mathrm{Ci} / \mathrm{mM}, 160 \mathrm{mCi} / \mathrm{ml}$; ICN Biochemicals, Inc., Irvine, CA, USA). The labeling was done in an Eppendorf tube by mixing $50 \mathrm{pmol}$ of the oligonucleotide, $5 \mu \mathrm{l}$ of the $10 \times \mathrm{T}_{4}$ polynucleotide kinase buffer $(0.5 \mathrm{M}$ Tris- $\mathrm{HCl}, \mathrm{pH} 7.6,100 \mathrm{mM} \mathrm{MgCl} 2,100 \mathrm{mM} \mathrm{B}$-mercaptoethanol), $50 \mu \mathrm{Ci} \gamma 32 \mathrm{p}-\mathrm{ATP}$, and $3-6 \mathrm{U}$ of cloned $\mathrm{T}_{4}$ polynucleotide kinase $30 \mathrm{U} / \mu \mathrm{T}$; USB) in a total volume of $50 \mu \mathrm{l}$, adjusted with water. The reaction mixture was incubated at $37^{\circ} \mathrm{C}$ for 1 hour.

The unincorporated radioactive $n$ ts were removed from the probes by using commercially available Nensorb (NEN) columns (NEN Products, DuPont) under the conditions recommended by the manufacturer. The column was washed with $2 \mathrm{ml} 100 \%$ methanol and equilibrated with $2 \mathrm{ml}$ reagent $A(0.1 \mathrm{M}$ Tris-HCl, $10 \mathrm{mM}$ triethylamine, $1.0 \mathrm{mM}$ disodium EDTA, $\mathrm{pH} \mathrm{7.7).} \mathrm{The} \mathrm{labeled}$ DNA mixture was diluted with $400 \mu l$ reagent $A$ and applied directly to the column. Next, the unincorporated radioactive nts were removed by washing the column with $2 \mathrm{ml}$ reagent $A$. The $5^{\prime}$ end-labeled oligonucleotide was eluted from the column with $500 \mu 1$ reagent $B$ (50\% methanol) and collected in an Eppendorf tube. The activity of the probes as CPM was determined in a scintillation counter (Beckman).

iii. Dot-Blot Hybridization: The duplicate membranes were prehybridized for 30 minutes at $55^{\circ} \mathrm{C}$ in separate, sealed plastic bags, with $10 \mathrm{ml}$ of a prehybridization solution containing $5 \times$ SSPE, $5 \times$ Denhardt's, $0.5 \%$ SDS $\left(20 \times\right.$ SSPE $=3.6 \mathrm{mM} \mathrm{NaCl}, 10 \mathrm{mM} \mathrm{NaH} 2 \mathrm{PO}_{4}, 20 \mathrm{mM} \mathrm{Na} 2 \mathrm{EDTA}, \mathrm{pH} 7.4 ; 50 \times$ Denhardt's $=1 \%$ Ficoll, $1 \%$ PVP, 1\% BSA $)$. The probe $\left(50-100 \times 10^{6} \mathrm{cpm}\right)$ was added to the particular membrane and the hybridization was conducted for 1 to 2 hours in a circulating water bath. The temperature of the hybridization depends upon the $T_{m}$ of the probe $\left[T_{m}=4 x(G+C)+2 x\right.$ $(A+T)]$. The membranes were washed twice with $2 \times$ SSPE, $0.1 \%$ SDS solution at room temperature for 10 minutes. The next washing was done with TMAC washing solution (3.0 M TMAC, $50 \mathrm{mM}$ Tris- $\mathrm{HCl}, \mathrm{pH} 8.0,2.0 \mathrm{mM}$ EDTA, $0.1 \%$ SDS) for 20 minutes at $42^{\circ} \mathrm{C}$, followed by another wash at $58^{\circ} \mathrm{C}$ for 30 minutes. The use of TMAC in a washing solution equilibrates the A-T and G-C bonding strength differences, thus allowing the use of the same temperature for stringent washes for probes of the same size (24). Following washings, 
the membranes were dried, wrapped in plastic $f i l m$, and exposed to Kodak $x$-ray film (Eastman Kodak) for 2 to 12 hours.

7/e HLA DQ $\alpha$ Typing. AmpliType TM HLA DQ $\alpha$ Forensic DNA Amplification and Typing Kit (Perkin Elmer Cetus) was used for the detection and identification of human leukocyte antigen (HLA) DQ a alleles. The test was primarily used for paternity studies. The kit distinguishes six alleles (HLA $D Q \alpha 1.1,1.2,1.3,2,3$, and 4) that define 21 different genotypes. The procedure was performed in three steps as recommended by the manufacturer: i) PCR amplification, ii) hybridization, and $i i i)$ color development.

i. PCR Amplification: The reaction mixtures contained $0.5 \mu \mathrm{g}(0.1$

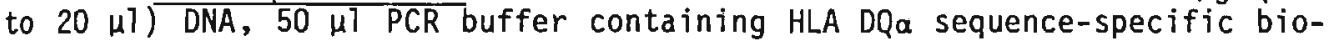
tinylated primers, AmpliTaq DNA polymerase, dATP, dCTP, dGTP, and dTTP, and $50 \mathrm{\mu l} 0.8 \mathrm{mM} \mathrm{MgCl} 2$. The samples were overlaid with mineral oil and subjected to 35 cycles of denaturation $\left(95^{\circ} \mathrm{C}\right.$ for 1 minute), annealing $\left(60^{\circ} \mathrm{C}\right.$ for 30 seconds), and extension $\left(72^{\circ} \mathrm{C}\right.$ for 30 seconds) on a Thermocycler (Perkin Elmer Cetus). The denaturation step in the first cycle and the extension step in the last cycle are prolonged for 4 and 7 minutes, respectively.

ii. Hybridization: Nine sequence specific DNA probes, which distinguish six HLA DQa alleles, are spotted onto a DNA probe strip that is part of the kit. DNA probe strips are placed in separate wells of the typing tray and $3.3 \mathrm{ml}$ hybridization-enzyme conjugate solution [3.3 $\mathrm{m}$ l hybridization solution ( $5 \times$ SSPE, $0.5 \%$ SDS) and $27 \mu l$ enzyme conjugate solution (horseradish peroxidase-streptavidin conjugate) per strip] are pipetted into each well. The PCR-amplified DNA is denatured at $95^{\circ} \mathrm{C}$ for 3 to 10 minutes, and immediately added to the contents of each well. The tray is covered by a clean plastic lid, and the amplified DNA is hybridized to the probes by incubation in a shaking water bath at $55^{\circ} \mathrm{C}$ for 20 minutes. Following hybridization, the contents of each well are aspirated and the strips rinsed with $10 \mathrm{ml}$ washing solution $(2.5 \times$ SSPE, $0.1 \%$ SDS $)$. The stringent wash is done with washing solution (10 $\mathrm{ml}$ per strip) in a shaking water bath at $55^{\circ} \mathrm{C}$ for 10 minutes, followed by another wash at room temperature for 5 minutes.

iii. Color Development: $10 \mathrm{ml} 0.1 \mathrm{M}$ sodium citrate buffer, pH 5.0, is pipetted into each we11, and the tray is placed on an orbital shaker at room temperature for 5 minutes. The color development solution is prepared 10 minutes before use by mixing $10 \mathrm{ml}$ sodium citrate buffer, $\mathrm{pH}$ 5.0, $10 \mathrm{ml} 3 \%$ hydrogen peroxide, and $0.5 \mathrm{ml}$ chromogen solution for each strip (chromogen solution $=30 \mathrm{mg}$ of $3^{\prime} 3^{\prime}, 5.5^{\prime}-$ TMB dissolved in $15 \mathrm{ml}$ reagent grade $100 \%$ ethanol). The citrate buffer is aspirated from the wells and $10 \mathrm{ml}$ of newly prepared color development solution is added to each well, the tray is covered with aluminum foil, and the color allowed to develop at room temperature for 20 to 30 minutes. The color development is stopped by washing the strips in deionized water. Fig. 2/5 shows the HLA DQ a typing results for two families with a child carrying an unstable $\mathrm{Hb}$. The results confirmed a de novo mutation in both probands. For details see Chapters $5 \mathrm{a}$ and $5 \mathrm{~b}$. 
A

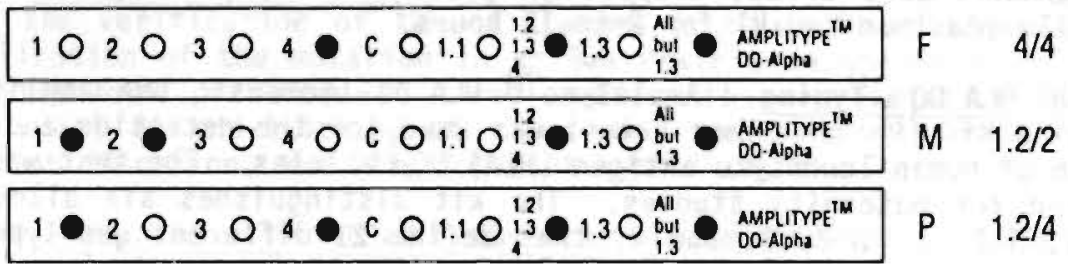

B

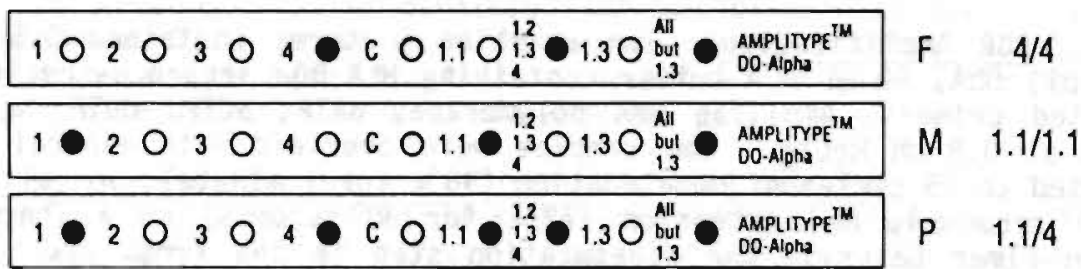

FIG. 2/5. HLA DQa typing results for two families with a child having an unstable Hb variant: $\mathrm{Hb}$ Yokohama [B 31(B13)Leu->Pro] (A), and $\mathrm{Hb}$ Volga $[B 27(B 9) A] a->A s p](B) . F=$ father; $M=$ mother, $P=$ propositus.

\section{References}

1. Righetti, P.G., Gianazza, E., Bianchi-Bosisio, A., and Cossu, G.: Conventional isoelectric focusing and immobilized $\mathrm{pH}$ gradients for hemoglobin separation and identification. In The Hemoglobinopathies, edited by T.H.J. Huisman, Methods in Hematology, Vol. 15, page 47, Churchill Livingstone, Edinburgh, 1986.

2. Bisse, E. and Wieland, H.: High-performance liquid chromatographic separation of human haemoglobins - Simultaneous quantitation of foetal and glycated haemoglobins. J. Chromatogr., 434:95, 1988.

3. Kutlar, A., Kutlar, F., Gu, L-G., Mayson, S.M., and Huisman, T.H.J.: Fetal hemoglobin in normal adults and $B$-thalassemia heterozygotes. Hum. Genet., 85:106, 1990.

4. Shelton, J.B., Shelton, J.R., and Schroeder, W.A.: High performance liquid chromatographic separation of globin chains on a large-pore $\mathrm{C}_{4}$ column. J. Liq. Chromatogr., 7:1969, 1984.

5. Kutlar, F., Kutlar, A., and Huisman, T.H.J.: Separation of normal and abnormal hemoglobin chains by reversed-phase high-performance liquid chromatography. J. Chromatogr., 357:147, 1986.

6. Efremov, G.D., Huisman, T.H.J., Bowman, K., Wrightstone, R.N., and Schroeder, W.A.: Microchromatography of hemoglobins. II. A rapid method for the determination of Hemoglobin $A_{2}$. J. Lab. Clin. Med., 83:657, 1974.

7. Betke, K., Marti, H.R., and Schlicht, I.: Estimation of small percentages of foetal haemoglobin. Nature, 184:1877, 1959.

8. Carre11, R.W. and Kay, R.: A simple method for the detection of unstable haemoglobins. Br. J. Haematol., 23:615, 1972.

9. Bucci, E. and Fronticelli, C.: A new method for the preparation of $\alpha$ and $B$ subunits of human hemoglobins. J. Biol. Chem., 240:551, 1965. 
10. Rosemeyer, M.A. and Huehns, E.R.: On the mechanism of dissociation of hemoglobin. J. Mol. Biol., 25:253, 1967.

11. Clegg, J.B., Naughton, M.A., and Weatherall, D.J.: Abnormal human haemoglobins. Separation and characterization of the $\alpha$ and $B$ chains by chromatography, and the determination two new variants, Hb Chesapeake and Hb-J-Bangkok. J. Mol. Biol., 19:91, 1966.

12. Huisman, T.H.J.: Separation of hemoglobins and hemoglobin chains by high-performance liquid chromatography. J. Chromatogr., 418:277, 1987.

13. Raftery, M.A. and Cole, R.D.: Tryptic cleavage at cysteinyl peptide bonds. Biochem. Biophys. Res. Commun., 10:467, 1963.

14. Jones, R.T.: Structural studies of aminoethylated hemoglobins by automatic peptide chromatography. Cold Spring Harbor Symp., Quant. Biol., 29:297, 1964.

15. Raftery, M.A. and Cole, R.D.: On the aminoethylation of proteins. J. Biol. Chem., 241:3457, 1966.

16. Wilson, J.B., Lam, H., Pravatmuang, P., and Huisman, T.H.J.: Separation of tryptic peptides of normal and abnormal $\alpha, \beta, \gamma$, and $\delta$ hemoglobin chains by high-performance liquid chromatography. J. Chromatogr., 179:271, 1979 .

17. Schroeder, W.A.: HPLC of globin chains and of peptides in the identification of hemoglobin variants. In The Hemoglobinopathies, edited by T.H.J. Huisman, Methods in Hematology, Vol. 15, page 143, Churchill Livingstone, Edinburgh, 1986.

18. Chang, J.Y., Brauer, D., and Wittmann-Liebold, B.: Micro-sequence analysis of peptides and proteins using 4-NN-dimethylaminoazobenzene 4'-isothiocyanate/phenylisothiocyanate double coupling method. FEBS Lett., 93:205, 1978.

19. Poncz, M., Solowiejczyk, D., Harpe1, B., Mory, Y., Schwartz, E., and Surrey, S.: Construction of human gene libraries from small amounts of peripheral blood: Analysis of B-like globin genes. Hemoglobin, $6: 27,1982$.

20. Saiki, R.K., Scharf, S., Faloona, F., Mullis, K.B., Horn, G.T., Erlich, H.A., and Arnheim, N.: Enzymatic amplification of the B-globin genomic sequences and restriction site analysis for diagnosis of sickle cell anemia. Science, 230:1350, 1985.

21. Saiki, R.K., Gelfand, D.H., Stoffel, S., Scharf, S.J., Higuchi, R., Horn, G.T., Mullis, K.B., and Erlich, H.A.: Primer directed enzymatic amplification of DNA with a thermostable DNA polymerase. Science, $239: 487,1988$.

22. Matteuci, M.D. and Caruthers, M.H.: Synthesis of deoxyoligonucleotides on a polymer support. Biotechnology, 24:92, 1992.

23. Sanger, F., Nicklen, S., and Coulson, A.R.: DNA sequencing with chain-terminating inhibitors. Proc. Nat1. Acad. Sci. USA, 74:5463, 1977.

24. Wood, W.G., Gitschia, J., Lasky, L.A., and Lawn, R.N.: Base composition independent hybridization in tetraethylammonium chloride. A method for oligonucleotide screening of highly complex gene libraries. Proc. Nat1. Acad. Sci. USA, 82:1585, 1985. 


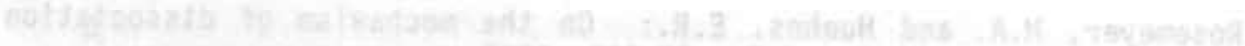

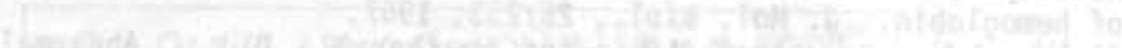

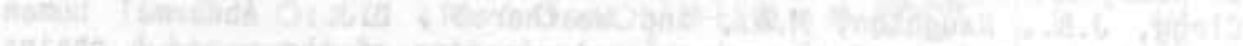

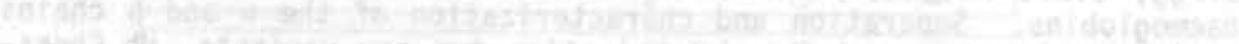

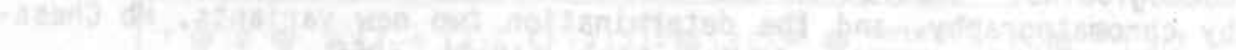

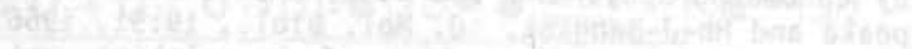

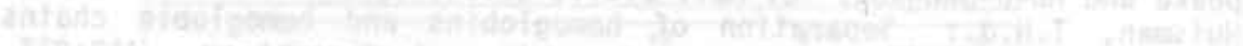

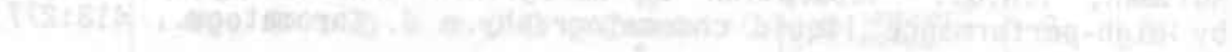

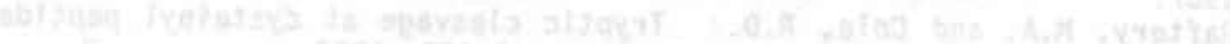

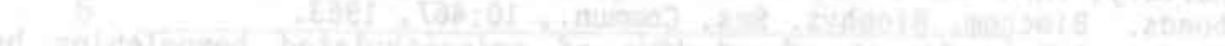

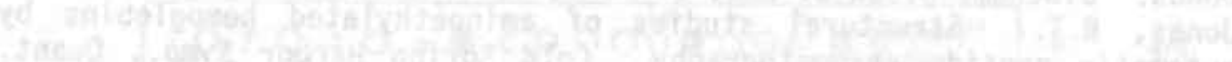

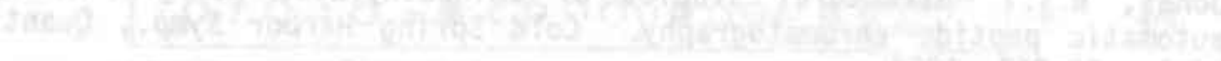

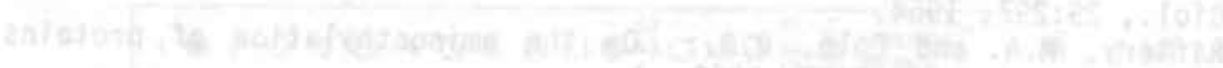

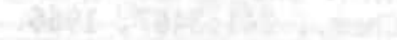

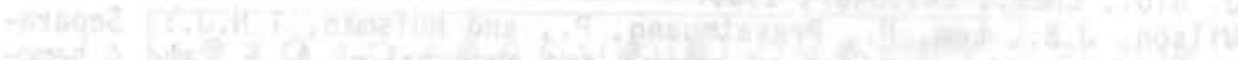

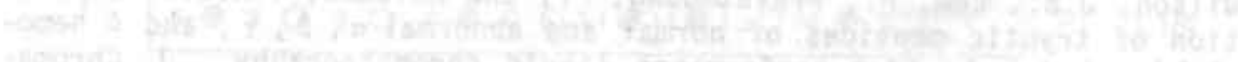

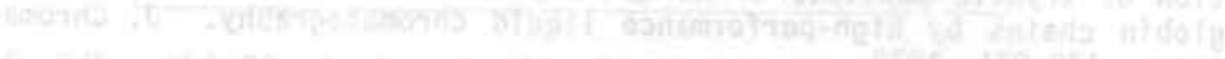

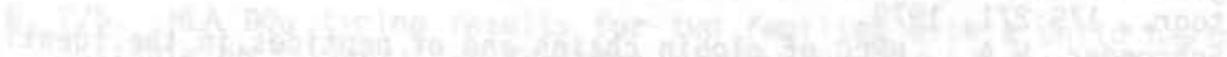

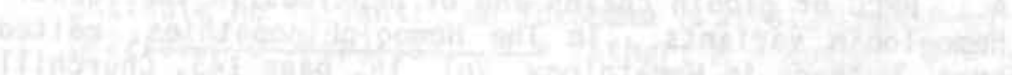

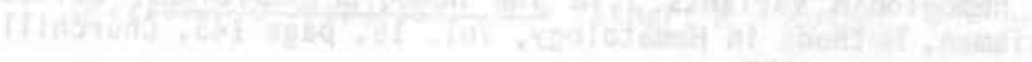

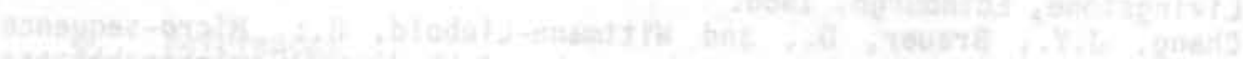

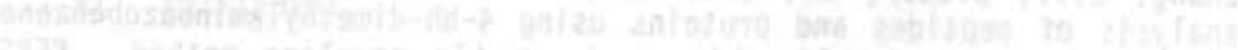

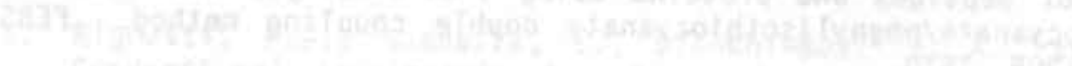

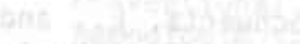

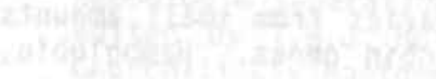

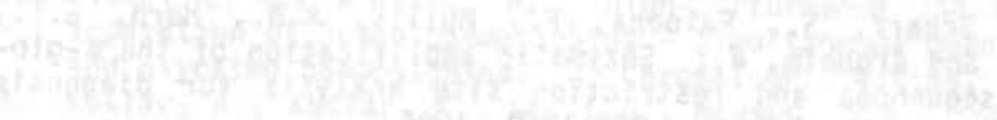

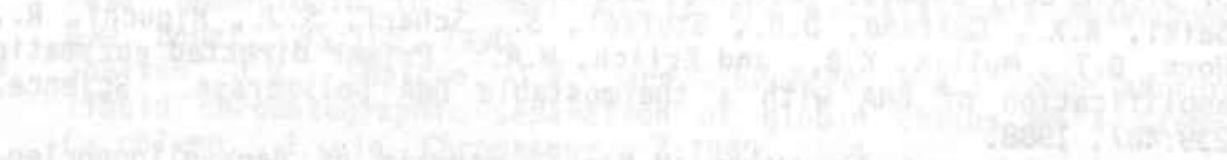

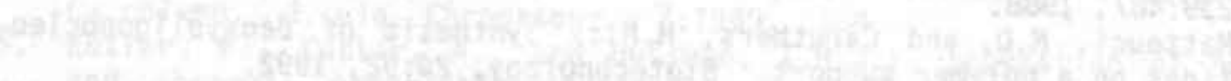

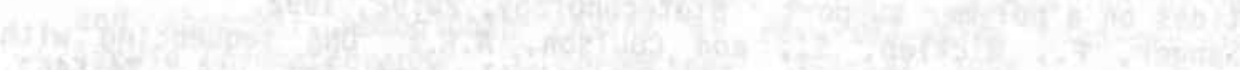

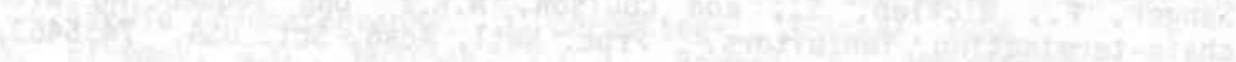

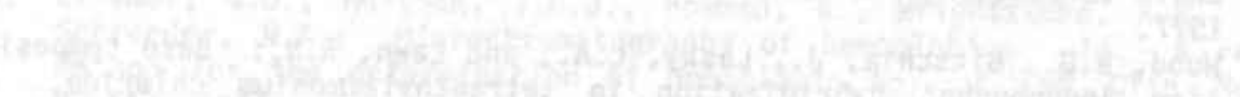

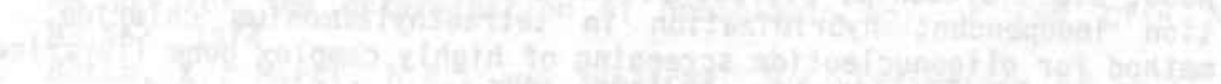

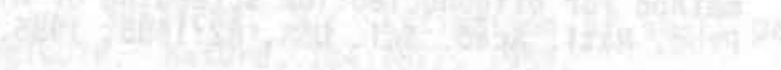


CHAPTER 3

\author{
a CHAIN VARIANTS \\ I DE N T I F I E D B Y H PLC
}

3a Hb Davenport or $\alpha_{2} 78\left(\right.$ EF7)Asn->His $\beta_{2}$

3b Hb Sun Prairie or $a_{2} 130(\mathrm{H} 13) \mathrm{Ala}->\mathrm{PrOB}_{2}$; Sec- 79 ond Observation in an Indian Adult 
ह

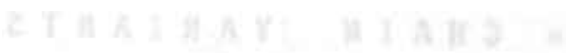

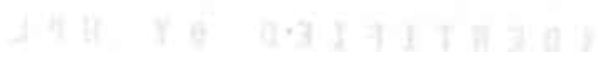


CHAPTER 3a

HB DAVENPORT OR $\alpha_{2} 78$ (EF7)ASN->HISB2

J.B. Wilson 1 , B.B. Webber ${ }^{1}$, D. Plaseskal, P.A. de Alarcon'2, S.K. McMillan2, and T.H.J. Huisman1

1 Department of Cell and Molecular Biology

Medical College of Georgia, Augusta, GA 30912-2100, USA

2 Iowa Department of Public Health, Des Moines, IA 50319-0075, USA

(Adapted from: Hemoglobin, 14:599-606, 1990)

\section{ABSTRACT}

Hb Davenport is a new, stable $\alpha$ chain variant, that was detected in two members of a Caucasian family living in Iowa. Hematological data were within normal limits. It is characterized by an Asn- $>$ His replacement at position $\alpha 78$. Mild acidic hydrolysis of the large aT-9 peptide, separation of the resulting fragments by reversed HPLC, and sequence analysis of a few of these peptides greatly facilitated the identification of this substitution. 


\section{INTRODUCTION}

of the 156 amino acid substitutions in the a chain of Hb listed in Ref. 1, 38 have been observed in the peptide $\alpha \mathrm{T}-9$. This large tryptic peptide contains 29 amino acid residues, occupies positions 62 through 90 of the a chajn and is characterized, among others, by high alanine and aspartic acid contents. Three $\mathrm{Hb}$ variants have been detected in which one of the seven alanine residues in this peptide is replaced by another amino acid, while some 20 variants have been described in which one of the six aspartic acid or asparagine residues is replaced by another amino acid (see Ref. 1 for details). The characterization of these variants is often complicated, and a distinction between substitutions such as Asp->His, AspAsn or Asn->Asp at positions $64,68,74,75,78$, and 85 is not easily made. A few years ago we introduced in our laboratory a relatively mild hydrolysis procedure (described below) in which the aT-9 peptide is hydrolyzed at the $N$ - and $C$-terminal ends of the aspartyl residues but not of the asparagine residues; the resulting smaller fragments are then separated by reversed phase HPLC and analyzed. With this method we have identified an Asn->His replacement at position a78 in a Hb variant found in a family from Iowa.

\section{MATERIALS AND METHODS}

Blood samples were collected in vacutainers with EDTA as anticoagulant and studied at the local institution and at the laboratories in Augusta, GA. Informed consent was obtained.

Hematological data were collected with automated cell counters. Red cell lysates were studied by IEF (2), citrate agar electrophoresis (3) by cation exchange HPLC $(4,5)$, and by reversed phase HPLC $(6,7)$. The abnormal a chain $(\alpha x)$ was separated from the normal $\alpha$ and $\beta$ chains by CM-cellulose chromatography (8), and digested with trypsin for 6 hours at room temperature and at $\mathrm{pH}$ 8.5. The resulting peptides were separated by reversed phase HPLC (9) and their amino acid compositions were determined by a Waters PicoTag amino acid analyzer system.

Peptide aT-9 was isolated from about $2 \mathrm{mg}$ of $a \times$ chain $(0.1-0.15$ $\mu$ Mole), dissolved in $2.0 \mathrm{ml} 2.2 \%$ formic acid ( $\mathrm{HCOOH}$ ) and hydrolyzed in a sealed tube under a mild vacuum for 5 hours at $110^{\circ} \mathrm{C}$ in a heating block (10). The resulting fragments were recovered by freeze-drying and separated by reversed phase HPLC with the developing system described in Ref. 11. The amino acid compositions of the isolated fragments were determined with the same amino acid analyzer, while sequence analysis of the amino termini of the two peptides was as described in Ref. 12 .

\section{RESULTS}

The propositus, an infant girl, was tested for the presence of an abnormal $\mathrm{Hb}$ through the facilities of the University of Iowa Hygienic Laboratory. She and her father had $a H b$ variant which moved between $H b A$ and 
$H b F$ in IEF and eluted slightly faster than $\mathrm{Hb}_{2}$ in cation exchange HPLC. The family is of German descent but has been established in Iowa for several generations. Hematological data for the father were most complete and were normal. The concentration of the variant was estimated at $20 \%$ ( $\mathrm{Hb} \mathrm{X}+\mathrm{Hb} \mathrm{A}_{2}$ was 22.3\%) and that of the slower eluting abnormal $\mathrm{Hb} \mathrm{A}$ (i.e. $\alpha_{2} X_{\delta_{2}}$ ) at $0.4 \%$. The $\alpha^{X}$ chain eluted slightly ahead of $a A$ on reversed phase HPLC.

Fig. $3 a / 1$ (top) illustrates the separation of the soluble peptides from a tryptic digest of the $a^{X}$ chain. All peptides except for the core peptide $\alpha T-12$, were recovered and identified. The a T-9 peptide was recovered as $\alpha T-9$, as $\alpha T-8,9$, as $\alpha T-9,10$, and even as $\alpha T-8,9,10$. The amino acid compositions of these four peptides (Table $3 a / 1$ ) readily identified the fragments and detected an Asp (or Asn)->His replacement.

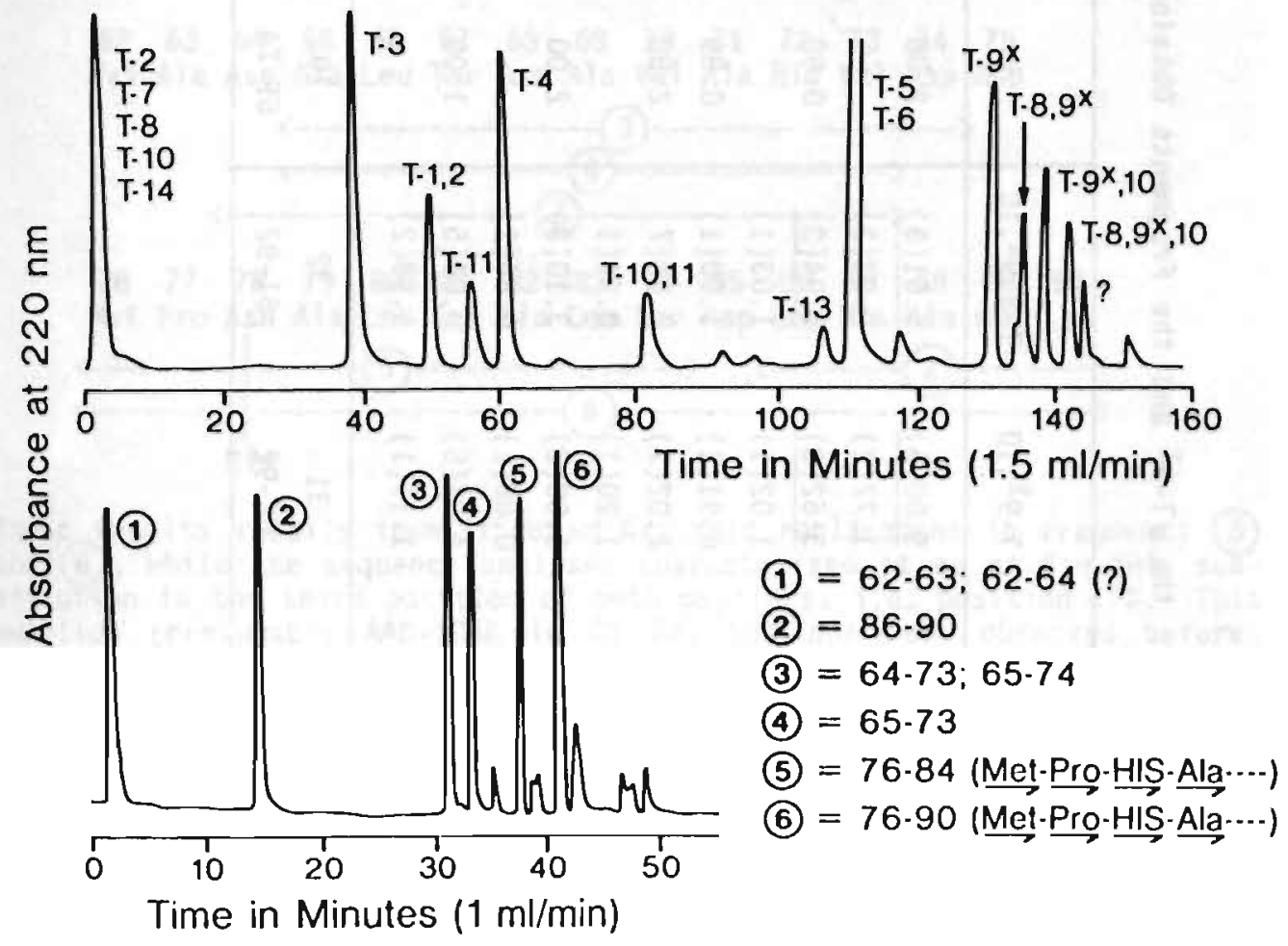

FIG. 3a/1. Top: Separation of the soluble peptides in a tryptic digest of the isolated $\alpha^{X}$ chain by reversed phase HPLC (9). Bottom: Separation of the fragments after mild acidic digestion of the a T-gX peptide by reversed phase HPLC (11). Amino acid composition data identified the locations of these fragments in the intact a chain (listed at the right).

Peptide aT-gX was also isolated from a second chromatogram and hydrolyzed with $2.2 \% \mathrm{HCOOH}$ as described. The separation of the fragments by reversed phase HPLC with the developers described by Schroeder (11) is 
TABLE 3a/1. Amino Acid Compositions of the a T-gX and the Fragments Obtained After Mild Hydrolysis With $2.2 \% \mathrm{HCOOH}^{\mathrm{a}}$

\begin{tabular}{|c|c|c|c|c|c|c|c|c|}
\hline \multirow[b]{2}{*}{ Amino Acid } & \multirow[b]{2}{*}{$T-9 X$} & \multirow[b]{2}{*}{$T-8,9 X$} & \multirow[b]{2}{*}{$T-9 X, 10$} & \multirow[b]{2}{*}{$T-8,9^{X}, 10$} & \multicolumn{4}{|c|}{ Fragments ${ }^{b}$} \\
\hline & & & & & 3 & 4 & 5 & 6 \\
\hline Aspartic Acid & $4.88(6)$ & $4.99(6)$ & $5.00(6)$ & $5.01(6)$ & 2.00 & 1.28 & $\underline{0}$ & $\underline{0.72}$ \\
\hline Serine & $1.71(2)$ & $1.77(2)$ & $1.77(2)$ & $2.03(2)$ & & & 1.89 & 1.88 \\
\hline Histidine & $3.72(3)$ & $3.87(3)$ & $3.62(3)$ & $3.94(3)$ & 0.98 & 0.98 & $\underline{1.00}$ & 3.01 \\
\hline Arginine & 0 & 0 & $1.02(1)$ & $1.03(1)$ & & & & \\
\hline Threonine & $0.88(1)$ & $0.87(1)$ & $0.91(1)$ & $1.00(1)$ & 0.89 & 0.90 & & \\
\hline Alanine & $7.00(7)$ & $7.07(7)$ & $7.02(7)$ & $7.04(7)$ & 2.80 & 2.90 & 2.06 & 2.80 \\
\hline Proline & $1.03(1)$ & $0.97(1)$ & $1.20(1)$ & $0.94(1)$ & & & 0.98 & 0.92 \\
\hline Valine & $2.91(3)$ & $3.00(3)$ & $3.00(3)$ & $3.10(3)$ & 2.00 & 2.05 & & \\
\hline Methionine & $0.86(1)$ & $0.89(1)$ & $0.88(1)$ & $0.86(1)$ & & & 0.67 & 0.75 \\
\hline Leucine & $4.01(4)$ & $4.01(4)$ & $4.94(5)$ & $5.04(5)$ & 1.00 & 1.01 & 2.13 & 2.98 \\
\hline Lysine & $1.00(1)$ & $2.00(2)$ & $1.11(1)$ & $1.99(2)$ & & & & 1.00 \\
\hline No. of Residues & 29 & 30 & 31 & 32 & 10 & 9 & 9 & 15 \\
\hline Positions in $a^{X}$ & $62-90$ & $61-90$ & $62-92$ & $61-92$ & $65-74$ & $65-73$ & $76-84$ & $76-90$ \\
\hline
\end{tabular}

a In moles/mole.

b See Fig. 3a/1 (bottom). 
shown in Fig. 3a/1 (bottom section), while the amino acid compositions of four fragments are listed in Table $3 a / 1$. Sequence analysis of the amino termini of the peptides in zones 5 and 6 located an Asn->His replacement in the third position of both.

\section{DISCUSSION}

The mild hydrolysis of peptide aT-9, followed by the complete separation of the resulting fragments by reversed phase HPLC, greatly facilitated the identification of the substitution in this a chain variant. The positions in the $\alpha$ chain of the five fragments isolated by this method, are shown in the following scheme. The circled numbers refer to the peaks in Fig. $3 a / 1$ (bottom) and to the amino acid compositions listed in Table 3a/1.

\section{$\begin{array}{llllllllllllll}62 & 63 & 64 & 65 & 66 & 67 & 68 & 69 & 70 & 71 & 72 & 73 & 74 & 75\end{array}$ \\ Val Ala Asp Ala Leu Thr Asn Ala Val Ala His Val Asp Asp}

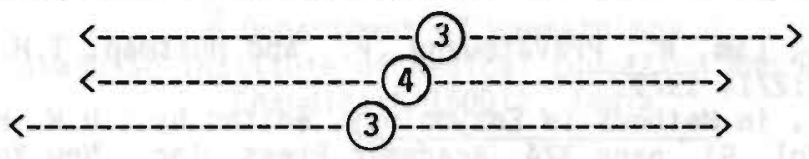

$\begin{array}{lllllllllllllll}76 & 77 & 78 & 79 & 80 & 81 & 82 & 83 & 84 & 85 & 86 & 87 & 88 & 89 & 90\end{array}$

Met Pro Asn Ala Leu Ser Ala Leu Ser Asp Leu His Ala His Lys

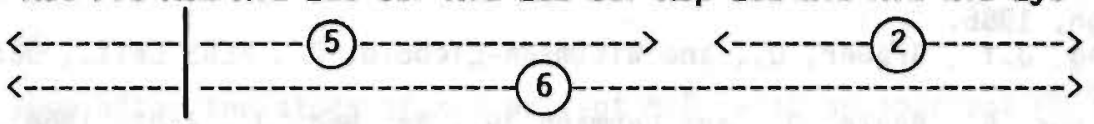

His

These results readily identified an Asx->His replacement in fragments (5) and (6), while the sequence analyses characterized it as an Asn->His substitution in the third position of both peptides; i.e. position $\alpha 78$. This mutation (presumably AAC- $>$ CAC in $C D$ 78) has not been observed before. The new variant was named $\overline{H b}$ Davenport after the city of residence of the family.

Hb Davenport is the 39th variant with a substitution of an amino acid residue in the $\alpha T-9$ peptide. Other variants in which the Asn residue is replaced are Hb Stanleyville-II (a78 Asn->Lys) (13) and Hb J-Singa (a78 Asn->Asp) (14). Asn $\alpha$ 78(EF7) is not involved in any major contact with the heme group or in contacts between chains, and its replacement by another amino acid is not likely to affect $\mathrm{Hb}$ stability. Hb Davenport carriers are not clinically affected by the presence of this variant.

Acknowledgements. This research was in part supported by USPHS Research Grant HLB-05168 and by Grant MCJ-191003 from the Bureau of Maternal and Child Health Resources and Development. This is contribution \#1273 from the Department of Cell and Molecular Biology at the Medical College of Georgia in Augusta, Georgia. 


\section{REFERENCES}

1. International Hemoglobin Information Center, Hemoglobin, $14: 249,1990$.

2. Righetti, P.G., Gianazza, E., Bianchi-Bosisio, A., and Cossu, G., in The Hemoglobinopathies, edited by T.H.J. Huisman, Methods in Hematology, Vol. 15, page 47, Churchill Livingstone, Edinburgh, 1986.

3. Huisman, T.H.J. and Jonxis, J.H.P., The Hemoglobinopathies Techniques of Identification, Clinical and Biochemical Analysis, Vol. 6, Marcel Dekker, Inc., New York, 1977.

4. Bisse, E. and Wieland, H., J. Chromatogr., 494:95, 1988.

5. Kutlar, A., Kutlar, F., Gu, L-G., Mayson, S.M., and Huisman, T.H.J., Hum. Genet., 86:106, 1990.

6. Shelton, J.B., Shelton, J.R., and Scliroeder, W.A., J. Liq. Chromatogr., $7: 1969,1984$.

7. Kutlar, F., Kutlar, A., and Huisman, T.H.J., J. Chromatogr., 357:147, 1986.

8. Clegg, J.B., Naughton, M.A., and Weatherall, D.J., J. Mol. Biol., 19: $91,1966$.

9. Wilson, J.B., Lam, H., Pravatmuang, P., and Huisman, T.H.J., J. Chromatogr., 179:271, 1979.

10. Inglis, A.S., in Methods in Enzymology, edited by C.H.W. Hirs and S.N. Tinlasheff, Vol. 91, page 324, Academic Press, Inc., New York, 1983.

11. Schroeder, W.A., in The Hemoglobinopathies, edited by T.H.J. Huisman, Methods in Hematology, Vol. 15, page 142, Churchill Livingstone, Edinburgh, 1986.

12. Chang, J.Y., Brauer, D., and Wittmann-Liebold, B., FEBS Lett., 93:205, 1978 .

13. Van Ros, G., Beale, D., and Lehmann, H., Br. Med. J., 4:92, 1968.

14. Wong, S.C., Ali, M.A.M., Pond, J.R., Rubin, S.M., Johnson, S.E.N., Wilson, J.B., and Huisman, T.H.J., Biochim. Biophys. Acta, 784:187, 1984 . 
CHAPTER 3b

\title{
HB SUN PRAIRIE OR a 130 (H13)ALA->PROB 2 ; SECOND OBSERVATION IN AN INDIAN ADULT
}

\author{
D. Plaseska1, L-H. Gu1, J.B. Wilson 1 , J.F. Codrington 1 , \\ T.H.J. Hui sman 1 , and S. Dash ${ }^{2}$ \\ 1 Laboratory of Protein Chemistry \\ Department of Cell and Molecular Biology \\ Medical College of Georgia, Augusta, GA 30912-2100, USA \\ 2 Department of Haematology \\ Postgraduate Institute of Medical Education and Research \\ Chandigarh-160012, India
}

(Adapted from: Hemoglobin, 14:491-497, 1990)

Soon after the study of the patient M.R. with an apparent Hb Sun Prairie homozygosity was completed and recorded (see Ref. 1) we observed the same a chain variant in a young 20 -year-old Indian male with clinical and hematological features of a chronic hemolytic anemia. His Hb level was $10.8 \mathrm{~g} / \mathrm{dl}$ and $\mathrm{PCV}$ value was $0.29 \mathrm{l} / 1$; a distinct reticulocytosis was present $(12 \%)$, while the blood smear was characterized by anisopoikilocytosis, microcytosis with hypochromia, and target cells. Heat stability tests were essentially negative. A modest icterus was noted and gallstones were detected by $X$-ray of his abdomen. The availability of a red cell lysate and isolated DNA permitted only limited studies, but these provided additional data important for the characterization of this new variant.

The Hb abnormality was undetectable by electrophoresis at alkaline $\mathrm{pH}$ and by IEF; the methods have been described before $(2,3)$. In a PolyCAT cation exchange HPL chromatogram $(4,5)$, a shoulder appeared on the Hb A zone, and the $\mathrm{Hb} \mathrm{A}_{2}$ zone appeared complex, suggesting the presence of additional $\mathrm{Hb}$ components (Fig. $3 \mathrm{~b} / \mathrm{l}$, top). The abnormal a chain was readily detected by reversed phase HPLC $(6,7)$ eluting well ahead of the normal a chain (Fig. 3b/1, bottom). The $\alpha X$ chain was isolated by preparative reversed phase HPLC (8) and studied as described (1). The tryptic peptide separation was similar to that shown in Fig. 3 of Ref. 1, except that a slower gradient allowed a complete separation of the $\alpha^{T}-13^{X}$ and $a T-5+$ a T-6 peptides. The amino acid composition of aT-13X was: Ser 2.90(3); Thr 1.84(2); Ala 0(1); Pro 0.99(0); Val 2.00(2); Leu 2.02(2); Phe 1.10(1); Lys $0.99(1)$, with values for the normal $\alpha$ T-13 between parentheses; this leaves no doubt that the only alanine residue in this peptide, a 130, is replaced by proline. 

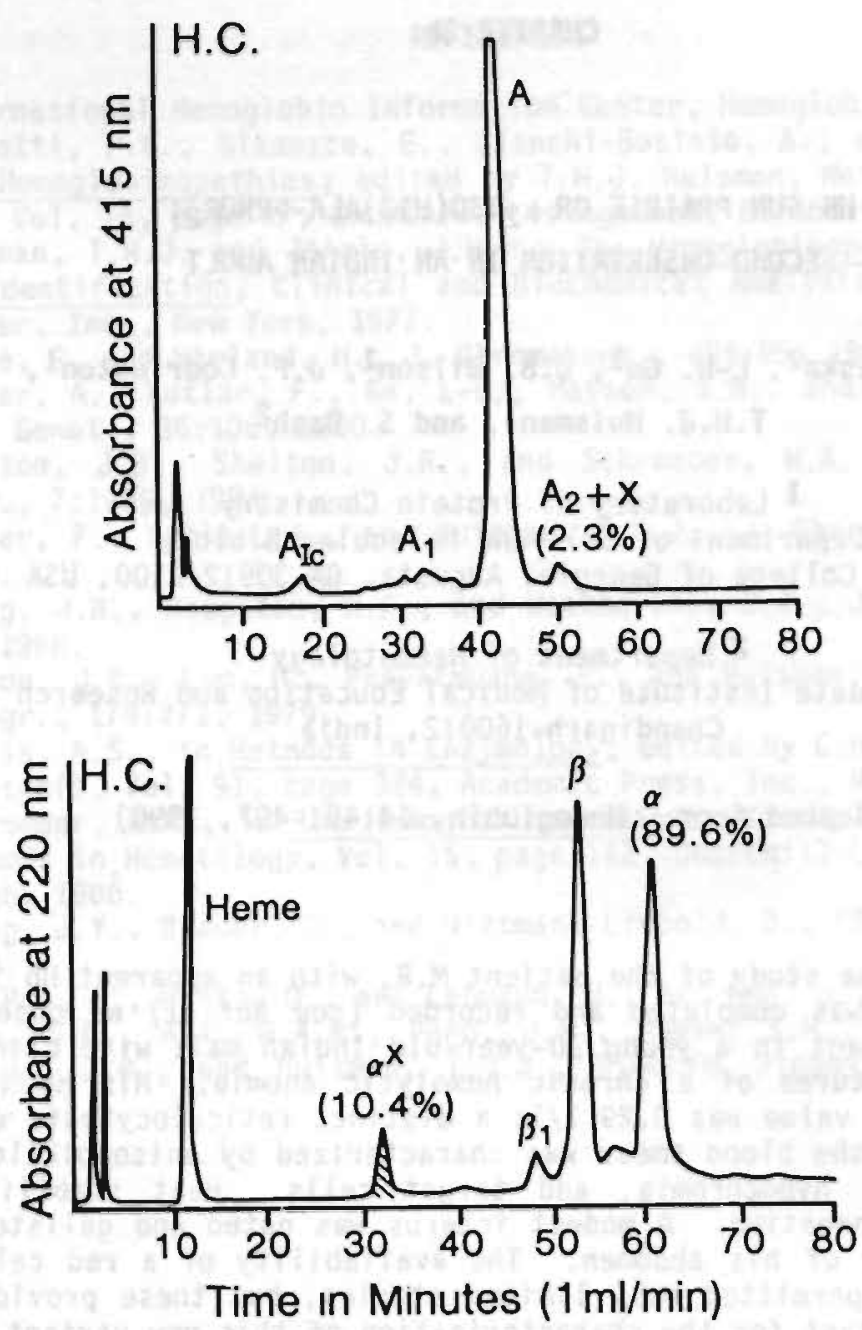

FIG. 3b/1. Top: Separation of the Hb fractions present in the whole red celT lysate of patient H.C. by PolyCAT cation exchange HPLC $(4,5)$. $\mathrm{Hb} \mathrm{X}_{2}$ is eluted slightly later than $\mathrm{Hb} \mathrm{A}_{2}$; separation is incomplete. Bottom: Separation of the globin chains present in a total red cell lysate of patient H.C. by reversed phase $\operatorname{HPLC}(6,7)$.

Because of the apparent heterogeneity of the $\mathrm{Hb} \mathrm{A}$ and $\mathrm{Hb} \mathrm{A}_{2}$ zones we decided to isolate these for further analysis. This was accomplished by preparative DEAE-cellulose chromatography $(9)$, which allows the rapid separation of these $\mathrm{Hbs}$ (Fig. 3b/2, top left). $\mathrm{Hb} \mathrm{A}_{2}$ and two zones of $\mathrm{Hb}$ A were eluted from this material and their chain composition was studied by reversed phase HPLC. The results are given in the three chromatograms which make up the remainder of $\mathrm{Fig} .3 \mathrm{~b} / 2$. The $\mathrm{Hb} \mathrm{A}_{2}$ zone contained no $B$ chain, but only $\delta, \alpha$, and $28 \% \alpha \times$. The two $H b$ A zones contained a considerabiy smaller quantity of $\alpha x$, and the values of $10.8-12.3 \%$ were nearly the same as that found in the whole red cell lysate (Fig. 3b/1, bottom). The slightly higher level of $a^{X}$ in fraction 2 of $H b A$ suggests a partial resolution of $\mathrm{HB} A$ and $\mathrm{Hb} X$. 

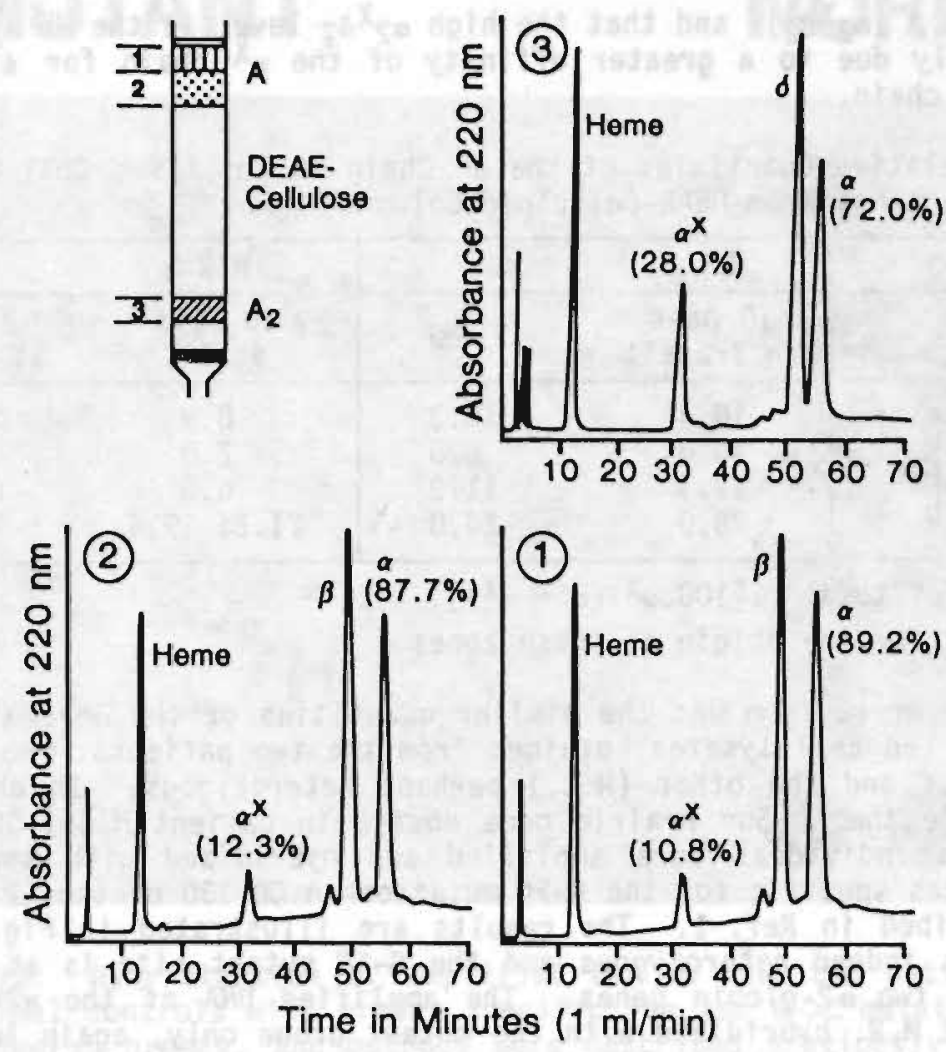

FIG. 3b/2. Top left: Diagram illustrating the separation of Hb A (plus $H b X$ ) and $H b A_{2}$ (plus $H b X_{2}$ ) on a DEAE-cellulose column (9). Fractions numbered 1,2 , and 3 were isolated and concentrated, and analyses by reversed phase $\operatorname{HPLC}(6,7)$ are presented.

This study of a second patient with the Hb Sun Prairie variant provided two surprising pieces of information. First, the level of the $\alpha^{x}$ chain (and thus of Hb Sun Prairie) in this Indian adult appeared to be higher than that of the child with an apparent homozygosity for this variant. Second, the relative quantity $a_{2} X_{\delta_{2}}$ in the $H b A_{2}$ zone (28\%) is about 2.5 times that of $\alpha_{2} X_{B 2}$ in the $H b$ A zone (11-12\%). This may be explained by: 1) A difference in stability with $\mathrm{Hb} \mathrm{X}_{2} \mathrm{x}_{2}\left(\mathrm{a}_{2} \mathrm{X}_{\delta_{2}}\right)$ having a near normal stability, or 2) a greater affinity of the $\alpha x$ chain for $\delta$ chains than for B chains.

Because of these new findings we decided to analyze a fresh blood sample from the child (M.R.) in whom Hb Sun Prairie was first discovered. The sample was collected in EDTA and shipped in wet ice by overnight courier to Augusta, GA where it was analyzed the next day and at various periods thereafter. The quantitative data are listed in Table $3 \mathrm{~b} / 1$. The total quantity of $a^{X}$ in the freshly collected sample was found to be $10.3 \%$ which is the same value as that observed in the hemolysate of the second patient H.C. (Table 3b/1). Aging of the sample resulted in a decrease of the $\alpha x$ levels in the total hemolysate and in all $\mathrm{Hb}$ fractions. This suggests that the stability of the abnormal $\mathrm{Hb}_{\mathrm{b}} \mathrm{A}_{2}\left(\alpha_{2} \mathrm{X}_{2}\right)$ is similar to that for 
the abnormal $\mathrm{Hb} A\left(\alpha_{2} X_{B_{2}}\right)$, and that the high $a_{2} x_{\delta_{2}}$ level in the $H b A_{2}$ fraction is probably due to a greater affinity of the $a^{X}$ chain for $\delta$ chains than for the $\beta$ chain.

TABLE 3b/1. Relative Quantities of the $a^{X}$ Chain in Total Red Cell Lysates and Hb Fractions From DEAE-Cellulose Columns ${ }^{a}$

\begin{tabular}{l|cc|cc}
\hline Patient ---> & H.C. & \multicolumn{2}{|c}{ M.R. } \\
\hline $\begin{array}{l}\text { Condition } \\
\text { of Sample }\end{array}$ & $\begin{array}{c}\text { 10 Days } \\
\text { in Transit }\end{array}$ & Fresh & $\begin{array}{c}10 \text { Days } \\
\text { at } 4{ }^{\circ} \mathrm{C}\end{array}$ & $\begin{array}{c}3 \text { Mos. } \\
\text { at }-70^{\circ} \mathrm{C}\end{array}$ \\
\hline Whole Lysate & 10.4 & 10.3 & 8.9 & 3.8 \\
A $_{1}$ (zone 1) & 10.8 & 8.8 & 2.0 & 0.2 \\
$A_{0}$ (zone 2) & 12.3 & 11.9 & 6.5 & 0.4 \\
A $_{2}$ (zone 3) & 28.0 & 24.0 & $21.2 ; 19.4$ & 9.5 \\
\hline
\end{tabular}

a Data in $\% \alpha^{X}$ of total $\left[100 \cdot \alpha^{X} /\left(\alpha+\alpha^{X}\right)\right]$.

b See Fig. $3 b / 2$ for the origin of these zones.

Another major concern was the similar quantities of the $\alpha^{X}$ chain (ca. 10-11\%) in the red cell lysates obtained from the two patients, one (M.R.) being homozygous and the other (H.C.) perhaps heterozygous. In order to further evaluate the $\mathrm{Hb}$ Sun Prairie gene dosage in patient M.C., DNA samples from each individual were amplified and hybridized with synthetic oligonucletotides specific for the $6->C$ mutation in CD 130 of the a2-globin gene, as described in Ref. 1 . The results are illustrated in Fig. $3 \mathrm{~b} / 3$. Patient H.C. is indeed heterozygous and the $\mathbf{G}->\mathbf{C}$ mutant site is at CD 130 of one of the two a 2-globin genes. The amplified DNA of the a2-globin gene of patient M.R. hybridized with the mutant probe only, again indicating a homozygosity for this mutation. The similar quantities of a-Sun Prairie present in these two patients are most unusual unless patient M.R. is not homozygous. Attempts to detect a normal $\alpha 2-g 10 b i n$ gene in patient M.R. have not been successful; selection of additional primers for amplification did not change the hybridization data, while sequence analysis of the $3^{\prime}$ 'segment (including the third exon) of the amplified DNA of the

2-globin gene did not reveal any mutant site which could prevent hybridization with the normal probe. Only the CCT sequence (for proline) was detected for CD 130. Thus, this problem remains unsolved and family studies are needed to further detail the abnormality in the child M.R., who, unfortunately, is adopted.

Acknowledgements. This study was supported by USPHS research Grant HLB-05168. This is contribution \#1274 from the Department of Cell and Molecular Biology at the Medical College of Georgia in Augusta, GA.

\section{REFEREMCES}

1. Harkness, M., Harkness, D.R., Kutlar, F., Kutlar, A., Wilson, J.B., Webber, B.B., Codrington, J.F., and Huisman, T.H.J., Hemoglobin, 14: $479,1990$. 


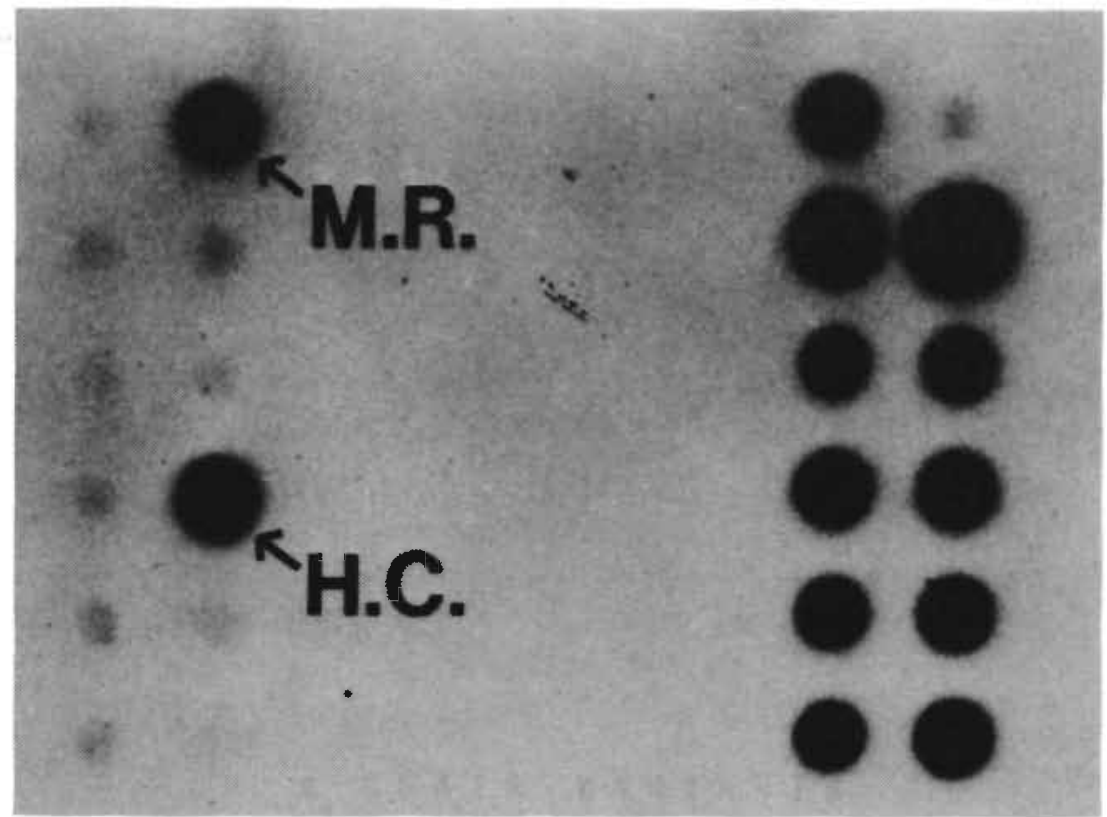

FI6. 3b/3. Hybridization of amplified DNA of the two patients and four normal controls with probes specific for the $G->C$ mutation at CD 130 . Primers, probes, and methods were described previously (1). The six samples at the left in each hybridization experiment contain DNA amplified from al-globin genes, and those at the right contain DNA amplified from $\alpha^{2}-g 10 b i n$ genes.

2. Huisman, T.H.J. and Jonxis, J.H.P., The Hemoglobinopathies Techniques of Identification, Clinical and Biochemical Analysis, Vol. 6, Marcel Dekker, Inc., New York, 1977.

3. Righetti, P.G., Gianazza, E., Bianchi-Bosisio, A., and Cossu, G., in The Hemoglobinopathies, edited by T.H.J. Huisman, Methods in Hematology, Vol. 15, page 47, Churchill Livingstone, Edinburgh, 1986.

4. Bisse, E. and Wieland, H., J. Chromatogr., 434:95, 1988.

5. Kutlar, A., Kutlar, F., Gu, L-G., Mayson, S.M., and Huisman, T.H.J., Hum. Genet., 85:106, 1990.

6. Shelton, J.B., Shelton, J.R., and Schroeder, W.A., J. Liq. Chromatogr., $7: 1969,1984$.

7. Kutlar, F., Kutlar, A., and Huisman, T.H.J., J. Chromatogr., 357:147, 1986.

8. Huisman, T.H.J., J. Chromatogr., 418:277, 1987.

9. Schroeder, W.A. and Huisman, T.H.J., The Chromatography of Hemoglobin, Clinical and Biochemical Analysis, Vol. 9, Marcel Dekker, Inc., New York, 1980. 


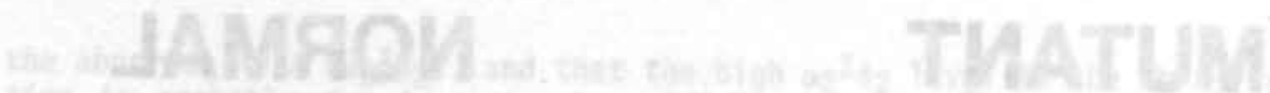

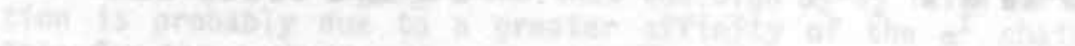

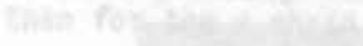

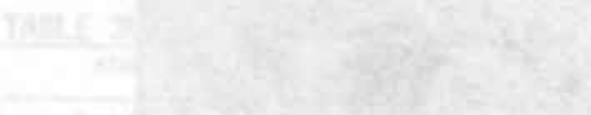

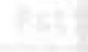

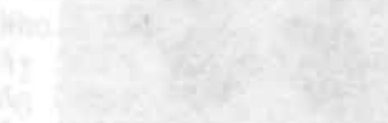

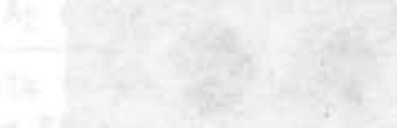

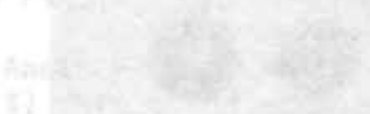

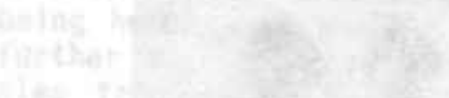

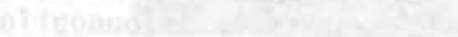

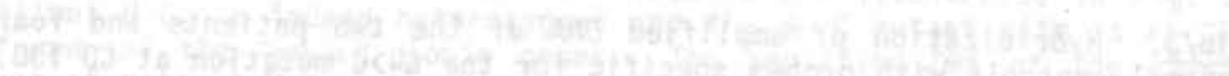

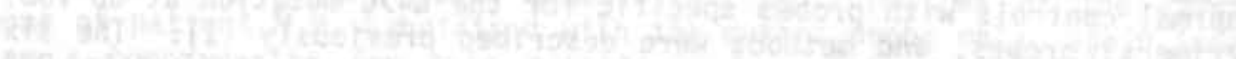

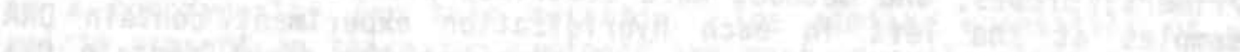

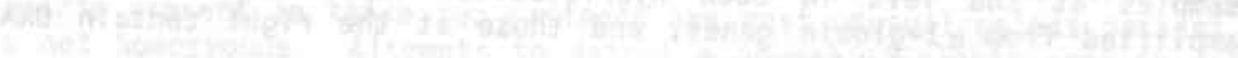

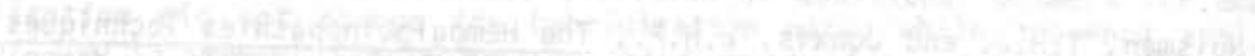

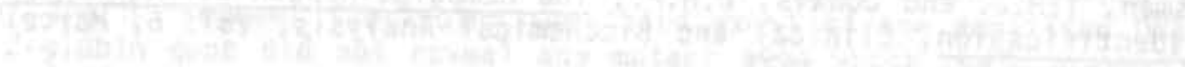

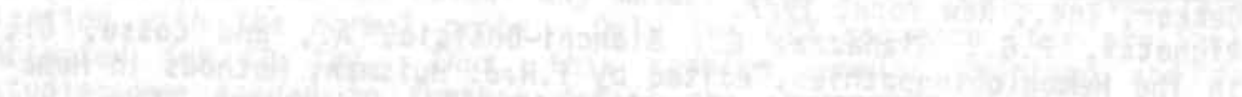

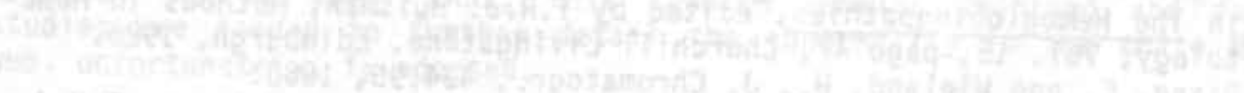

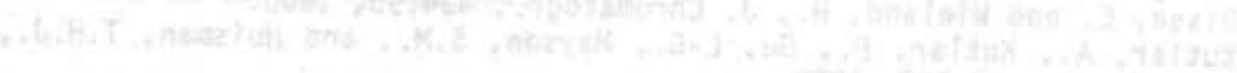

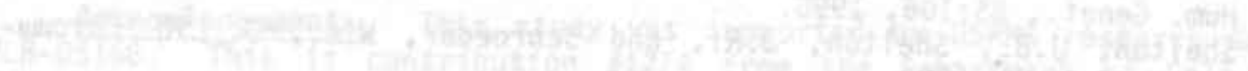

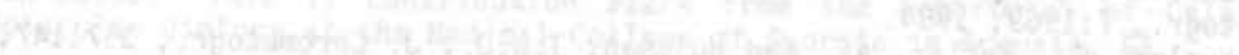

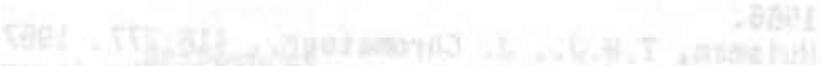

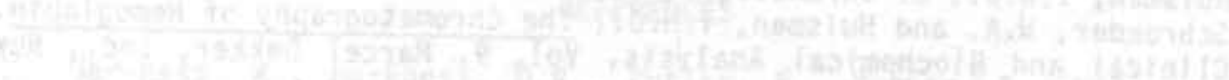


CHAPTER 4

$$
\begin{aligned}
& \text { B CHAIN VARIANTS } \\
& \text { I DENTIFIED BY HPLC }
\end{aligned}
$$

4a Hb Iowa or a $2^{\beta} 2^{119(6 H 2) T r p->A r g} 87$

4b Hb Zengcheng or $a 2^{\beta} 2^{114(616) L e u->M e t ~} 93$ 


\section{CHAPTER 4a}

HB IOHA OR a $2^{B} 2^{119(G H 2) G L Y->A L A ~}$

D. Plaseska1, P.A. de Alarcon2, S. McMillan²,

M. Walbrecht ${ }^{3}$, J.B. Milson 1 , and T.H.J. Huisman 1

1 Department of Cell and Molecular Biology

Medical College of Georgia, Augusta, GA 30912-2100, USA

2 Sickle Cell and Major Hemoglobinopathies

Department of Pediatrics, University of lowa Hospitals and Clinics

Iowa City, IA 52242 , USA

3 University Hygienic Laboratory, Des Moines, IA 50319, USA

(Adapted from: Hemoglobin, 14:423-429, 1990)

\section{ABSTRACT}

Hb Iowa with a Gly->Ala mutation at position $\beta 119$ (GH2) was observed in a $\mathrm{Bl}$ ack infant and her mother. The baby was also heterozygous for $\mathrm{Hb} \mathrm{S}$; $\mathrm{Hb}$ Iowa was confused with $\mathrm{Hb} \mathrm{F}$ at birth because its electrophoretic mobility was similar to that of $\mathrm{Hb} F_{1}$. The $B$ chain of $\mathrm{Hb}$ Iowa could be readily separated from the $\beta A, \alpha$, and $\gamma$ chains by PAGE and by reversed phase HPLC. Structural characterization was through amino acid analyses of peptides isolated from a tryptic digest of the aminoethylated B-Iowa chain. The Gly->Ala replacement in $\mathrm{Hb}$ Iowa does not affect its stability and oxygen carrying properties; hematological data for mother and child were within normal ranges. 


\section{INTRODUCTION}

The Iowa State-wide screening and care program for patients with significant hemoglobinopathies was established in May of 1987 and has identified several subjects with different forms of sickle cell disease. Neonatal testing has been initiated, and dry, whole blood filter paper specimens collected for the Newborn Metabolic Screen, constitute the initial samples which are analyzed by IEF using the eluted hemolysate. Abnormalities are confirmed from anticoagulated blood specimens obtained by venipuncture. Through this activity a Black female infant was suspected of having SS but upon repeat testing was identified as a compound heterozygote for $H b S$ and a variant with a mobility in IEF between $H b A$ and $H b F$. This report describes the identification of this variant and some of its properties.

\section{MATERIALS AND METHODS}

Blood samples from the propositus and her mother were collected in vacutainers with EDTA as anticoagulant, and studied at the local institutions and at the laboratories in Augusta, GA. Informed consent was obtained.

Hematological data were collected with automated cell counters and with standard laboratory procedures. Electrophoresis was with cellulose acetate at alkaline $\mathrm{pH}$ and citrate agar at $\mathrm{pH} 6.1$ (1), and with IEF (2) using agar plates which are commercially available from Isolab, Inc. (Akron, OH, USA). Quantitation of the various Hb fractions was by cation exchange HPLC using a PolyCAT column $(3,4)$. The mobilities of the individual chains were evaluated by reversed phase HPLC using the large-pore Vydac $C_{4}$ column $(5,6)$ and by PAGE $(7)$. Oxygen affinity measurements were made with a Hemox Analyzer (Southampton, PA, USA). A solubility test to confirm the presence of $\mathrm{Hb} F$ followed an established procedure (1).

A larger quantity of $H b X$ was isolated from the red cell lysate of the propositus by DEAE-cellulose chromatography (1); the mobility of $H b X$ in this system is the same as that of $\mathrm{Hb} A$. The $\alpha$ and $\beta$ chains were separated by CM-cellulose chromatography $(8)$ and the abnormal $\beta X$ chain was aminoethylated (9). Next, the $A E-\beta X$ chain was digested with trypsin for 6 hours at room temperature and at $\mathrm{pH} 8.7$. The resulting tryptic peptides were separated by reversed phase HPLC (10) and their amino acid compositions were determined with a Waters Pico Tag amino acid analyzer.

\section{RESIILTS}

The Family. When last studied, the propositus was a 9-month-old Black baby. Analysis of her red cell lysate by IEF revealed the presence of $\mathrm{Hb} S$ and $\mathrm{Hb} X$ which had a mobility slightly slower than that of $\mathrm{Hb} A$. Her mother had the same variant in combination with Hb A. Hematological data, listed in Table $4 \mathrm{a} / 1$, were within normal limits. A Heinz body preparation on the mother's blood was negative. A solubility test on the hemolysate of the child confirmed the presence of an $\mathrm{Hb} S$ trait. 
TABLE $4 a / 1$. Hema tological Data

\begin{tabular}{lccccccc}
\hline Subject & $\begin{array}{c}\text { Sex- } \\
\text { Age }\end{array}$ & $\begin{array}{c}\mathrm{Hb} \\
\mathrm{g} / \mathrm{dl}\end{array}$ & $\begin{array}{c}\mathrm{PCV} \\
\mathrm{l} / 1\end{array}$ & $\begin{array}{c}\mathrm{RBC} \\
1012 / 1\end{array}$ & $\begin{array}{c}\mathrm{MCV} \\
\mathrm{fl}\end{array}$ & $\begin{array}{c}\mathrm{MCH} \\
\mathrm{pg}\end{array}$ & $\begin{array}{c}\mathrm{MCHC} \\
\mathrm{g} / \mathrm{dl}\end{array}$ \\
\hline T.W. & F-9 mos. & 11.2 & 0.350 & 3.88 & 90 & 28.9 & 32.0 \\
V.W. & F-adult & 12.1 & 0.385 & 4.39 & 88 & 27.6 & 31.4 \\
\hline
\end{tabular}

Properties of the Variant. Heat stability and oxygen affinity analyses were made on the red cell lysate of the mother, which contained about the same quantities of $\mathrm{Hb} \mathrm{A}$ and $\mathrm{Hb} X$, and that of a normal control; no differences were observed. Fig. $4 a / 1$ illustrates the separation of the $\mathrm{Hb}$ components by cation exchange HPLC. Hb $X$ eluted at the same position as $\mathrm{Hb} A$. The level of $\mathrm{Hb} S$ in the propositus was slightly higher than that of $\mathrm{Hb} X$. Both the propositus and her mother had normal levels of $\mathrm{Hb} \mathrm{A}_{2}$, and the level of $7.2 \% \mathrm{Hb} \mathrm{F}$ in the lysate of the propositus is not unusual for a child of that age.
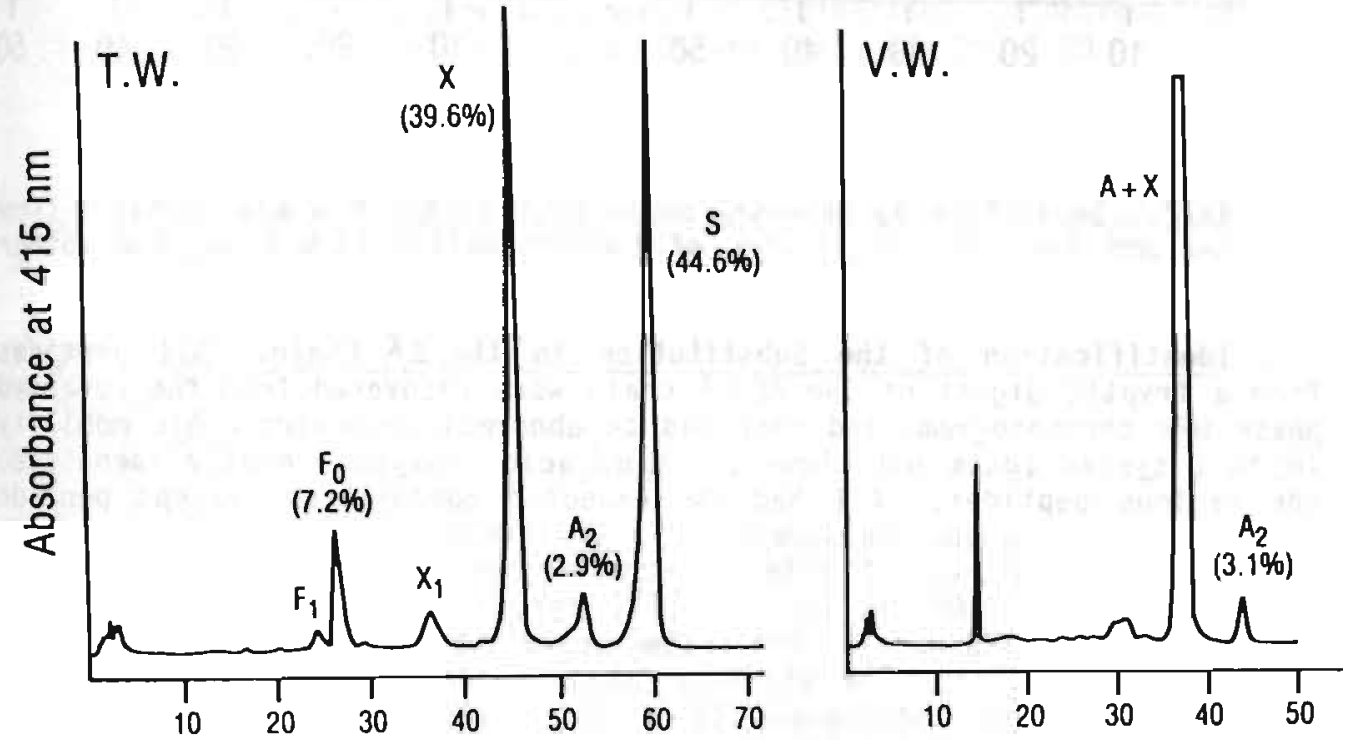

Time in Minutes $(0.8 \mathrm{ml} / \mathrm{min})$

FIG. 4a/1. Separation by cation exchange $\operatorname{HPLC}(3,4)$ of the Hbs in red cell lysates of the propositus (T.W.) and her mother (V.W.). The gradients used for the elution of the Hbs were slightly different in the two experiments.

The separation of the globin chains was evaluated by PAGE and reversed phase HPLC. With both techniques it was possible to detect normal a chains and abnormal $\beta$ chains. The $\beta^{A}$ and $\beta X$ chains were readily detected in the red cell lysate of the mother because the mobility of ${ }_{\beta} X$ in PAGE was slightly less than that of $B A$. The separation of the $\beta S$ and ${ }^{X} X$ chain in the child's lysate was even more evident because the mobility of ${ }_{B} S$ in this system was slightly greater than that of $B A$. Fig. $4 a / 2$ illustrates 
the mobilities of the three $B$ chains in reversed phase HPL chromatography. The ${ }_{B} X$ chain eluted just ahead of the normal $B$ chain but a complete separation of these two chains could not be obtained.
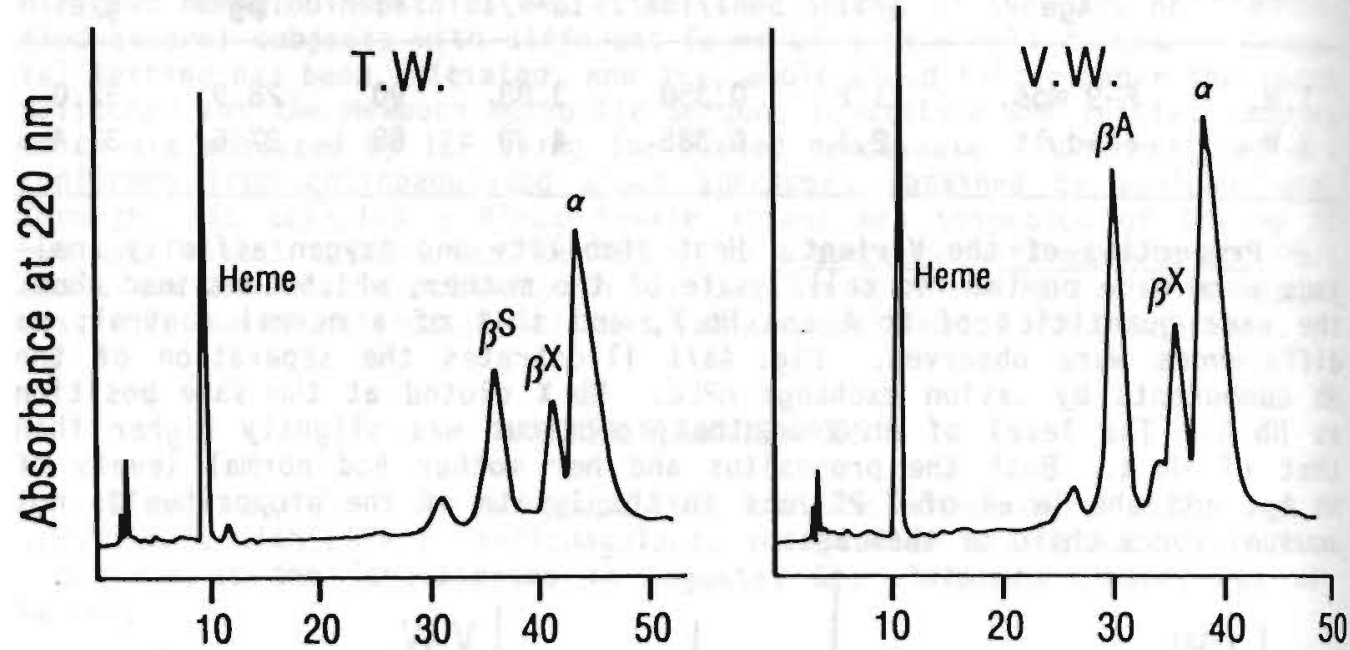

Time in Minutes $(1.0 \mathrm{ml} / \mathrm{min})$

FIG. 4a/2. Separation by reversed phase $\operatorname{HPLC}(5,6)$ of $\alpha$ and $\beta$ chains from the Hbs in red cell lysates of the propositus (T.W.) and her mother (V.W.).

Identification of the Substitution in the ${ }_{B} X$ Chain. All peptides from a tryptic digest of the $A E-\beta X$ chain were recovered from the reversed phase HPL chromatogram, and none had an abnormal chromatographic mobility in this system (data not shown). Amino acid analyses readily identified the various peptides. All had the expected compositions except peptide T-12B which showed the replacement of a glycine residue by an alanine residue. The composition of this octapeptide was: Gly 0(1); His 2.21(2); Ala 1.96(1); Val 1.00(1); Leu 0.92(1); Phe 0.94(1); Lys $0.96(1)$; values for the normal $\mathrm{T}-12 \mathrm{~B}$ peptide are listed between parentheses; data are given in mole/mole peptide. The Gly->Ala substitution occurs at position 7 in this peptide which corresponds to position 119 of the intact $\beta$ chain. This new variant was named Hb Iowa.

\section{COMMENTS}

Glycine at position 119, which is the second position of the interhelical region between helices $G$ and $H$, is involved in the $\alpha 1 B$ l contact mainly through residue 111 of the a chain (11). Substitution of glycine at $\beta 119$ by aspartic acid has been observed in Hb Fannin-Lubbock $(12,13)$ and by valine in $\mathrm{Hb}$ Bougardirey-Mali (14). Both variants had near normal functional and physicochemical properties; only a slight instability was observed. Similar data were obtained for Hb Iowa; the Gly->Ala replacement appears to have no effect at all, as even the heat stability of this variant was normal. Thus, the replacement of this glycine residue by various 
amino acids (Asp, Val, Ala) is not detrimental to the function of the $\mathrm{Hb}$ molecule, despite its involvement in molecular contacts between the $\alpha 1$ and B1 chains.

Acknowledgements. This research was supported in part by a grant from the Bureau of Maternal and Child Health and Resources Development MCJ-191003 (to PAdA), and by USPHS research grant HLB-05168 (to THJH). This is contribution \#1244 from the Department of Cell and Molecular Biology at the Medical College of Georgia in Augusta, GA.

\section{REFERENCES}

1. Huisman, T.H.J. and Jonxis, J.H.P., The Hemoglobinopathies Techniques of Identification, Clinical and Biochemical Analysis, Vol. 6, Marcel Dekker, Inc., New York, 1977.

2. Righetti, P.G., Gianazza, E., Bianchi-Bosisio, A., and Cossu, G., in The Hemoglobinopathies, edited by T.H.J. Huisman, Methods in Hematology, Vol. 15, page 47, Churchill Livingstone, Edinburgh, 1986.

3. Bisse, E. and Wieland, H., J. Chromatogr., 434:95, 1988.

4. Kutlar, A., Kutlar, F., Gu, L-G., Mayson, S.M., and Huisman, T.H.J., Hum. Genet., 85:106, 1990.

5. Shelton, J.B., Shelton, J.R., and Schroeder, W.A., J. Liq. Chromatogr., $7: 1969,1984$.

6. Kutlar, F., Kutlar, A., and Huisman, T.H.J., J. Chromatogr., 357:147, 1986.

7. Efremov, G.D., Markovska, B., Stojanovski, N., Petkov, G., Nikolov, N., and Huisman, T.H.J., Hemoglobin, 5:637, 1981.

8. Clegg, J.B., Naughton, M.A., and Weathera11, D.J., J. Mol. Biol., 19: $91,1966$.

9. Jones, R.T., Cold Spring Harbor Symposium, Quant. Biol., 29:297, 1964.

10. Wilson, J.B., Lam, H., Pravatmuang, P., and Huisman, T.H.J., J. Chromatogr., 179:271, 1979.

11. Sack, J.S., Andrews, L.C., Magnus, K.A., Hanson, J.C., Rubin, J., and Love, W.E., Hemoglobin, 2:153, 1978.

12. Moo-Penn, W.F., Bechtel, K.C., Johnson, M.H., Jue, D.L., Therre11, B.L., Morrison, B.Y., and Schmidt, R.M., Biochim. Biophys. Acta, 453: $472,1976$.

13. Schneider, R.G., Berkman, N.L., Brimhal1, B., and Jones, R.T., Biochim. Biophys. Acta, 453:478, 1976.

14. Chen-Marotel, J., Braconnier, F., Blouquit, Y., Martin-Caburi, J., Kammerer, J., and Rosa, J., Hemoglobin, 3:253, 1979. 


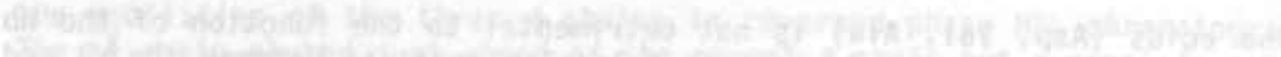

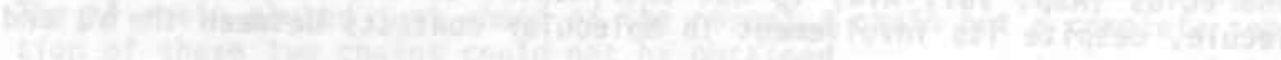

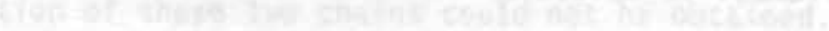

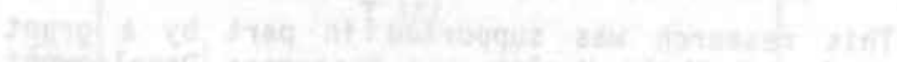

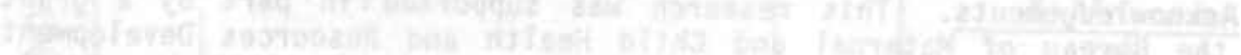

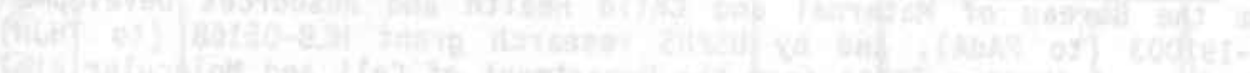

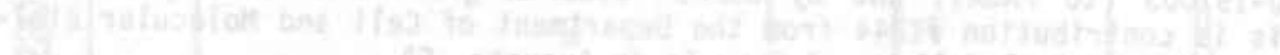

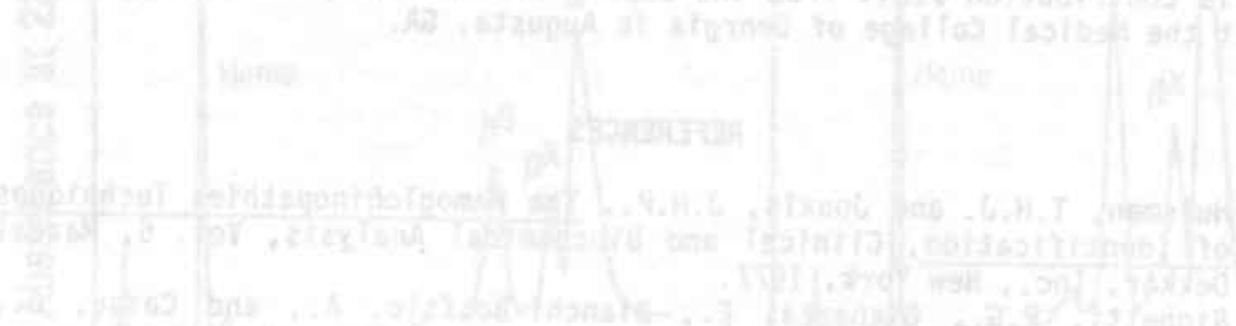

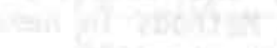
Th-

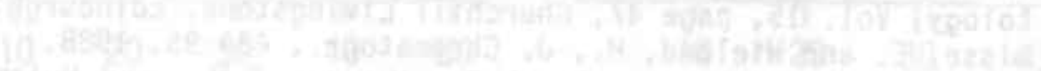

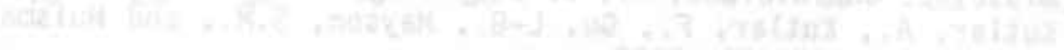

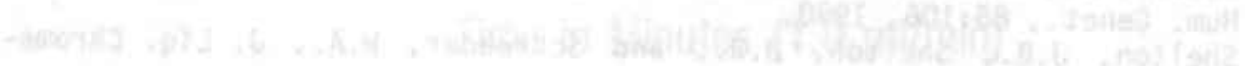

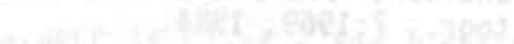

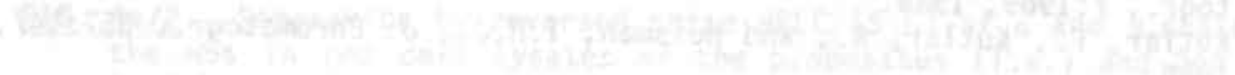

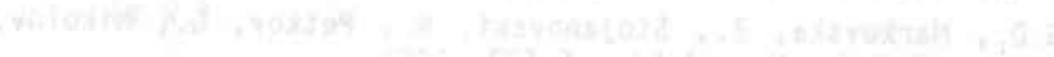

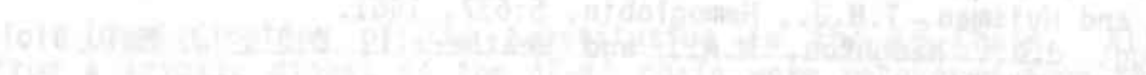

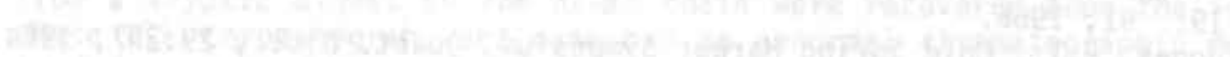
Q

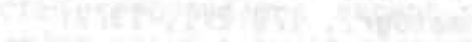

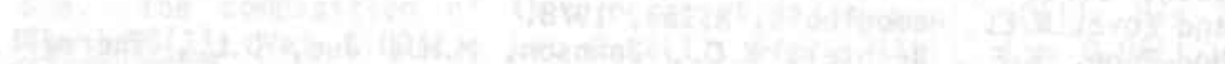

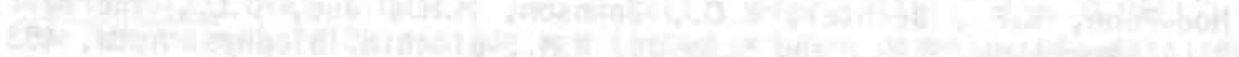
and

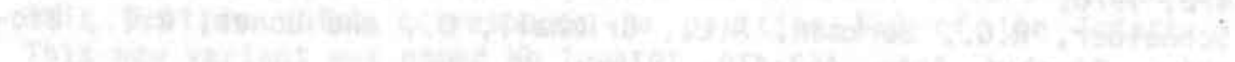

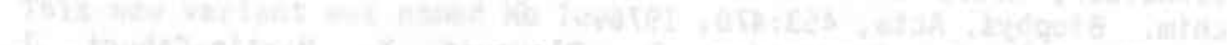

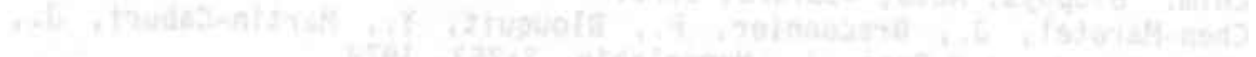

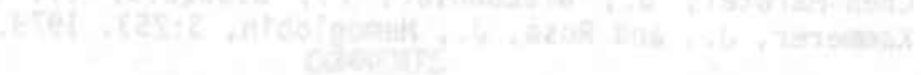


CHAPTER 4b

HB ZENGCHENG OR $\alpha_{2^{\beta} 2^{114}}$ (G16)LEU->MET

D. Plaseska1, J.B. Wilson'1, L-H. Gul, F. Kutlarl, T.H.J. Hui sman ${ }^{1}, Y-T$. Zeng ${ }^{2}$, and $M$. Shen ${ }^{2}$

1 Laboratory of Protein Chemistry Department of Cell and Molecular Biology

Medical College of Georgia, Augusta, GA 30912-2100, USA

2 Laboratory of Medical Genetics

Shanghai Children's Hospital, Shanghai, P.R. China

(Adapted from: Hemoglobin, 14:555-557, 1990)

The use of refined methodology, such as IEF (1), cation exchange HPLC $(2,3)$, and reversed phase HPLC $(4,5)$, as procedures for testing for the possible presence of $\mathrm{Hb}$ abnormalities has recently led to the detection of several new variants. This was again the case with $\mathrm{Hb}$ Zengcheng which was observed when a large batch of cord blood samples from Chinese newborns was tested by IEF and reversed phase HPLC.

Cord blood sample \#833 was collected from a normal Chinese baby originating in the city of Zengcheng in Guangdong Province, P.R. China. Examination of the Hb by IEF (1) showed the presence of a slightly fast-moving $\beta$ chain variant, of which the $\beta^{X}$ and $\beta^{A}$ chains were readily separated by reversed phase HPLC (5) (Fig. 4b/1). A quantity of the $\beta{ }^{X}$ chain was isolated by preparative HPLC using a $\dot{C}_{18}$ column (6). This ${ }_{\beta} X$ (estimated at $\sim 2 \mathrm{mg}$ ) was aminoethylated (7), digested with trypsin for 6 hours at room temperature and at $\mathrm{pH} 8.2$, and the resulting peptides were separated by a reversed phase HPLC procedure (8). All expected peptides were recovered but only peptide T-12B had a different chromatographic mobility and eluted slightly faster than the $T-12 B$ peptide of the normal ${ }_{B} A$ chain. Its amino acid composition was: Gly 1.07(1); His 1.62(2); Ala 1.14(1); Met 0.63(0); Leu $0(1)$; Phe $0.85(1)$; Lys $0.92(1)$; values for T-12B of the normal $B A$ chain are listed between parentheses. Sequence analysis with a manual micro procedure (9) gave a Val-Met-Ala- amino terminal sequence, confirming a Leu->Met substitution at position 114 of the $B$ chain. This mutation has not been observed before, and the variant is named $\mathrm{Hb}$ Zengcheng or $\alpha_{2}^{\beta}{ }_{2}^{114}$ (G16)Leu->Met. 


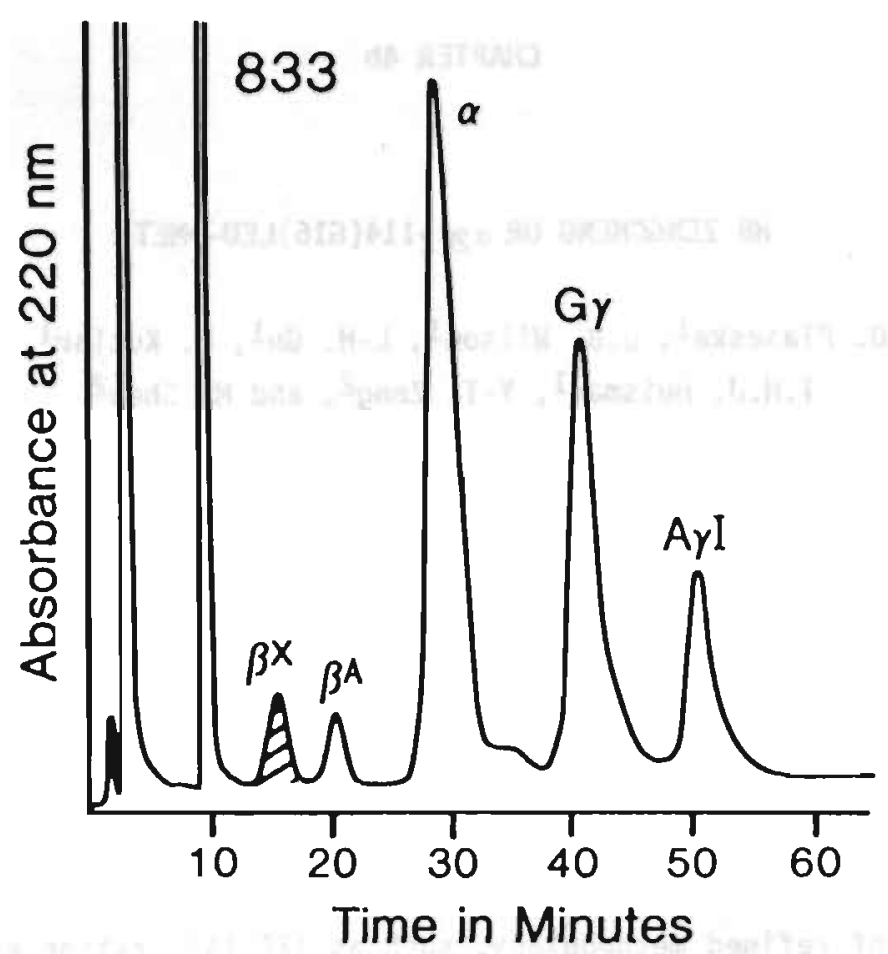

FIG. 4b/1. Separation of the $\beta^{X}, \beta^{A}, \alpha$, and $\gamma$ chains, present in a cord blood red cell lysate of the $\mathrm{Hb}$ Zengcheng heterozygote, by reversed phase HPLC. The quantities of the ${ }_{\beta} X$ and ${ }_{\beta} A$ chains were estimated at $3.8 \%$ and $3.0 \%$, respectively, of the total protein. The ratio between $\mathrm{G}_{Y}$ and $\mathrm{A}_{Y}$ was normal.

The leucine residue at position $B 114$ is internally located and is not involved in any major contacts with heme or globin subunits. Its replacement by another hydrophobic residue (methionine) is not expected to alter its physicochemical or functional properties. Family studies and a second analysis of the $\mathrm{Hb}$ Zengcheng heterozygote were not possible.

Acknowledgements. This research was supported by USPHS research grants HLB-05168 (to THJH) and HLB-29623 (to Y-TZ). This is contribution 11251 from the Department of Cell and Molecular Biology at the Medical College of Georgia in Augusta, GA.

\section{REFERENCES}

1. Righett1, P.G., Gianazza, E., Bianchi-Bosisio, A., and Cossu, G., in The Hemoglobinopathies, edited by T.H.J. Huisman, Methods in Hematology, Vol. 15, page 47, Churchill Livingstone, Edinburgh, 1986.

2. Bisse, E. and Wieland, H., J. Chromatogr., 434:95, 1988.

3. Kutlar, A., Kutlar, F., Gu, L-G., Mayson, S.M., and Huisman, T.H.J., Hum. Genet., 85:106, 1990. 
4. Shelton, J.B., Shelton, J.R., and Schroeder, W.A., J. Liq. Chromatogr., 7:1969, 1984.

5. Kutlar, F., Kutlar, A., and Huisman, T.H.J., J. Chromatogr., 357:147, 1986.

6. Huisman, T.H.J., J. Chromatogr., 418:277, 1987.

7. Jones, R.T., Cold Spring Harbor Symposium, Quant. Biol., 29:297, 1964.

8. Wilson, J.B., Lam, H., Pravatmuang, P., and Huisman, T.H.J., J. Chromatogr., 179:271, 1979.

9. Chang, J.Y., Brauer, D., and Wittmann-Liebold, B., FEBS Lett., 93:205, 1978 . 



\section{CHAPTER 5}

B CHAIN VARIANTS IDENTIFIED

BY HPLC AND D A ANALYSES

5a Hb Yokohama [B31(B13)Leu->Pro] Detected as a De Novo Mutation in a Yugoslavian Boy

5b Hb Volga [B 27(B9)Ala->Asp]: Detection of a De Novo Mutation by Ava II Digestion of PCRAmplified DNA 
2.

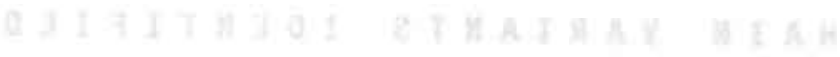

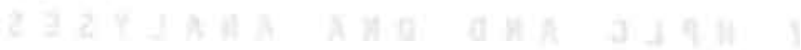

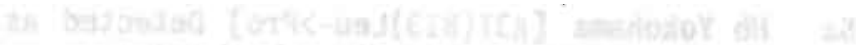

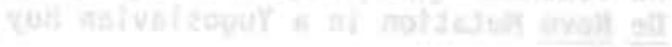

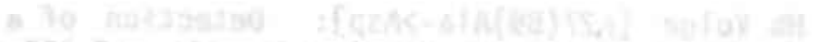

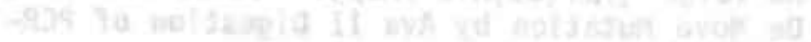

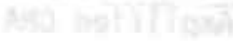




\title{
CHAPTER 5a
}

\section{HB YOKOHAMA [B31(B13)LEU->PRO] DETECTED AS A DE NOVO MUTATION IN A YUGOSLAVIAN BOY}

\author{
D. Plaseskal, L. Jankovic ${ }^{1}$, A.J. Dimovski ${ }^{1}$,
}

D. Milenovic ${ }^{2}$, D. Juricic ${ }^{3}$, and G.D. Efremov 1

1 Macedonian Academy of Sciences and Arts

Research Center for New Technologies, 91000 Skopje, Yugoslavia

\section{Department of Pediatrics}

Faculty of Medicine, 18000 Nis, Yugoslavia

3 Department of Clinical Chemistry

University Hospital Dr. O. Novosel, 41000 Zagreb, Yugoslavia

(Adapted from: Hemoglobin, 15:469-476, 1991)

\section{ABSTRACT}

Hb Yokohama [B31(B13)Leu->Pro] was observed in a young Yugoslavian boy as a de novo mutation. The child exhibited severe transfusion-dependent hemolytic anemia. The variant was detected and quantitated at $10.5 \%$ by reversed phase HPLC. Structural characterization of the abnormal $B$ chain was done by HPLC analysis, on material obtained by high salt precipitation. The mutation was confirmed by sequencing of the amplified DNA. 


\section{INTRODUCTION}

Hb Yokohama [B31(B13)Leu->Pro] was first described in 1981 by Nakatsuji et al (1). The variant was found in a 33-year-0ld Japanese woman with a history of chronic hemolytic anemia and in her son who manifested milder symptoms. Here we describe a second observation of this variant in a 10-year-old Yugoslavian boy suffering from hemolytic anemia.

\section{MATERIALS AND METHODS}

Hematological data were obtained with an automated cell counter and with routine hematological procedures. Red cell lysates were studied by starch gel electrophoresis at alkaline $\mathrm{pH}(2)$, and with PAGE in the presence of urea, acid, and Triton $X-100$ (3). The percentages of $H_{b} A_{2}$ and Hb $F$ were determined by microcolumn chromatography (4) and alkali denaturation procedures (5), respectively. The abnormal $B$ chain was quantitated by reversed phase HPLC (6). The stability of the abnormal Hb was determined by heat stability and PCMB tests (7). The abnormal Hb was isolated by precipitation with sodium chloride at $50^{\circ} \mathrm{C}$ for 60 minutes ( 1 vol. $5 \mathrm{gr}$ \% hemolysate, 2 vol. $0.1 \mathrm{M}$ Tris buffer, $\mathrm{pH} 6.0,1$ vol. $4 \mathrm{M} \mathrm{NaCl}$ ). The abnormal $\beta$ chain was separated from the $\alpha$ chain by $C M-52$ column chromatography (8). The isolated $\beta X$ chain was digested with trypsin for 5 hours at room temperature and at $\mathrm{pH}$ 8.6. The resulting peptides were separated by reversed phase HPLC (9). One zone was rechromatographed by a similar procedure (10). The amino acid compositions of the peptides were determined with a Beckman 6300 amino acid analyzer.

DNA was isolated from the leukocytes by the method of Poncz et al (11). The 710 bp fragment of the B-globin gene was amplified (12) and directly sequenced (13).

\section{RESULTS}

The propositus is a 10-year-old Yugoslavian boy living in Nis. His anemia was noted when he was 7 months of age. A few months later, he was hospitalized because of severe anemia and subicterus. At that time he was diagnosed as having a hereditary spherocytosis and was transfused for the first time. Anisocytosis, poikilocytosis, and microspherocytosis were noted on the peripheral blood smear. Liver and spleen were palpable 4 and $5 \mathrm{~cm}$ below the costal margin, respectively. Thereafter, he was hospitalized and transfused several times due to acute hemolytic crisis. He was splenectomized at the age of 2.5 years. Ever since then he has received several blood transfusions during acute infectious episodes. The first time he was sent to our institution was in 1988. Hematological data for the propositus, obtained before and after splenectomy, are given in Table $5 a / 1$. Peripheral blood smear revealed anisocytosis, poikilocytosis, target cells, and basophilic stippling. Heinz bodies were readily detectable after incubation of the blood at $37^{\circ} \mathrm{C}$ for 2 hours. The heat stability test was positive suggesting the presence of $14-16 \%$ unstable $H b$. The PCMB test was also positive; the precipitate consisted of $\beta$ chains with a normal 
mobility. No abnormal $\mathrm{Hb}$ was detected by starch gel electrophoresis at alkaline pH, PAG electrophoresis, and anion and cation exchange chromatography. An abnormal $B$ chain $(10.5 \%)$ was detected by a reversed phase HPLC procedure (Fig. $5 \mathrm{a} / 1$ ). The $\mathrm{Hb} \mathrm{A}_{2}$ value of $4.9 \%$ was higher than normal; $\mathrm{Hb} F$ was also increased (4.5\%). The parents of the boy were normal without any $\mathrm{Hb}$ abnormalities.

TABLE 5a/1. Hematological Data of the Propositusa

\begin{tabular}{|c|c|c|}
\hline & $\begin{array}{c}\text { Before Splenectomy } \\
1 / 83-4 / 83\end{array}$ & $\begin{array}{c}\text { After Splenectomy } \\
4 / 88-1 / 90\end{array}$ \\
\hline 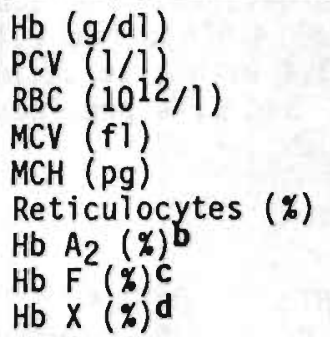 & $\begin{array}{c}4.8-7.2 \\
\text { n.d. } \\
2.8-3.1 \\
\text { n.d. } \\
17.7-25.4 \\
6.2-9.7 \\
\text { n.d. } \\
\text { n.d. } \\
\text { n.d. }\end{array}$ & $\begin{array}{c}8.9-10.9 \\
0.286-0.330 \\
2.9-3.6 \\
99.4-106.6 \\
26.4-34.2 \\
5.5 \\
4.6-4.9 \\
3.0-4.5 \\
10.5\end{array}$ \\
\hline
\end{tabular}

a Values obtained before transfusion.

b By microcolumn chromatography.

$C$ By alkali denaturation.

d By reversed phase HPLC.

n.d. = not determined.

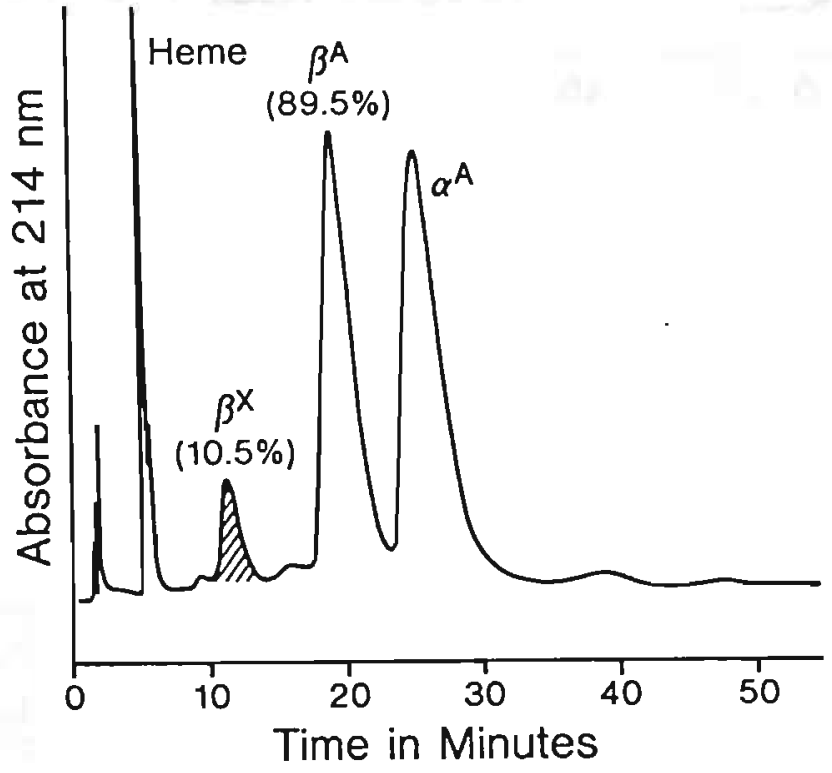

FI6. 5a/1. Separation of the $\beta^{X}, \beta_{B} A$, and $a^{A}$ chains, present in a red ce 11 lysate of the propositus, by reversed phase HPLC. 
Structural analyses were done by a reversed phase HPLC procedure. The tryptic peptides of the ${ }_{\beta} X$ chain are shown in Fig. $5 a / 2$. Almost all peptides were recovered with normal mobilities and normal amino acid compositions. Peptide BT-3 was recovered as a pcak much smaller than normally observed, while peptide $\beta T-4$ was nearly absent. The small zone, present at the position of the normal BT-4 peptide, probably originated from the small amount of normal $B$ chain that was present in the precipitate. An abnormal peptide eluted together with peptide $\beta T-8,9$. This aberrant peptide was identified as $\beta^{X} T-3,4$ because the substitution of proline for leucine produced a Lys-Pro linkage that trypsin does not cleave. Rechromatography was used to separate the $B T-8,9$ and $B T-3,4$ peptides. The amino acid composition of the abnormal peptide was: Asp 2.1(2); Thr 1.0(1); Glu 3.3(3); Pro 2.1(1); Gly 3.0(3); Ala 1.1(1); Val 4.6(5); Leu 2.1(3); Tyr $0.7(1)$; $\operatorname{Arg} 2.0(2)$; thus identifying it as $\beta{ }^{2} T-3,4$ with a substitution of Leu->Pro (values for residues of the normal $B T-3$ and $B T-4$ peptides are listed between parentheses).

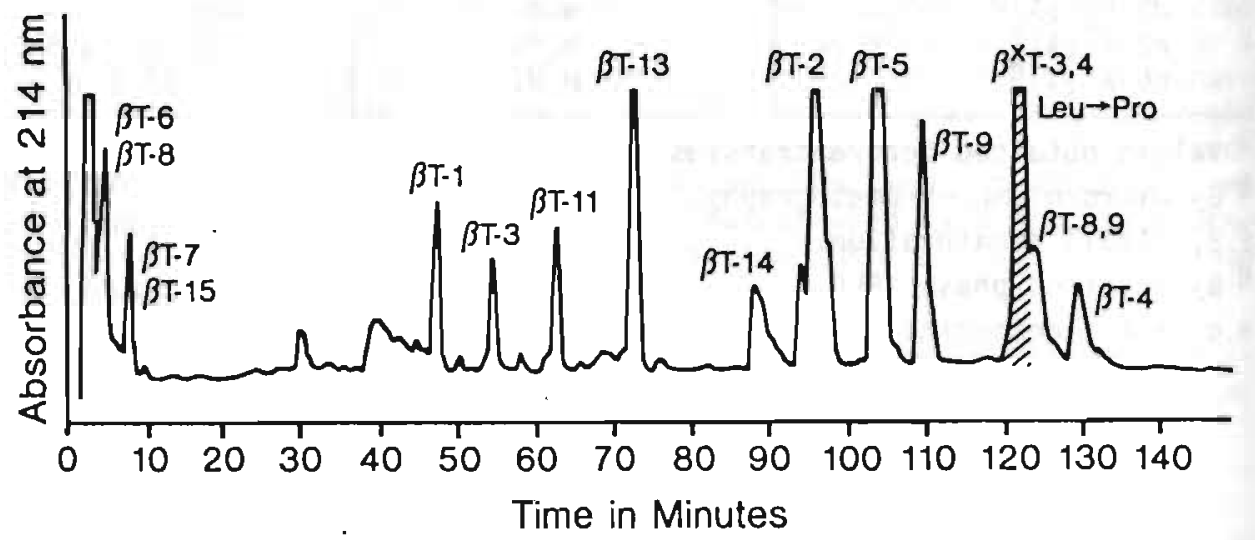

FIG. 5a/2. Separation of the tryptic peptides of the ${ }_{\beta} X$ chain by reversed phase HPLC.

The substitution was confirmed and localized at codon 31 through sequencing of amplified DNA. Two primers (synthesized on a Beckman System 1 DNA Synthesizer) were used to amplify a 710 bp fragment that was sequenced in both directions. Fig. $5 a / 3$ illustrates the results of the direct sequencing; two nucleotides ( $T$ and $C$ ) are present at the second position of codon 31 , resulting in a CIG->CCG (or Leu->Pro) mutation.

Since the parents of the boy did not have this abnormality, paternity studies were undertaken. The polymorphisms in the HLA DQa locus were evaluated using the Perkin Elmer Cetus HLA DQ a Forensic DNA Amplification and Typing Kit. The results revealed genotype $1.1 / 1.1$ for the mother, 4.4 for the father, and $1.1 / 4$ for the propositus, thus confirming the parentage of the propositus. 


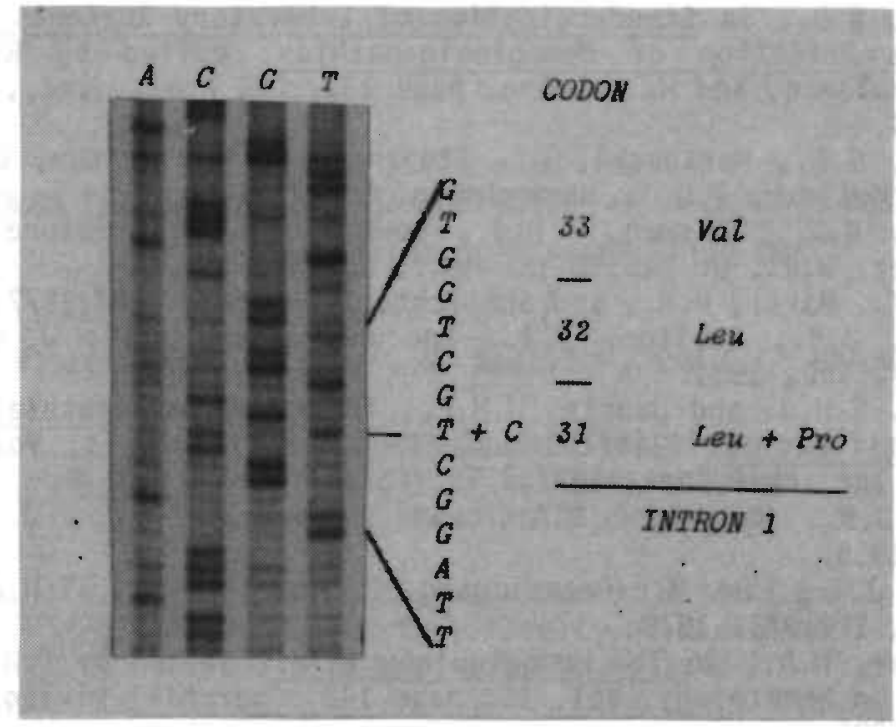

FIG. 5a/3. Direct sequence analysis of the amplified DNA of the propositus. Two nucleotides $(T+C)$ are present at the second position of codon 31. The sequences of the amplification primers (numbers given in parentheses are relative to the Cap site) were: Direct: 5'-GCCAAGGACAGGTACGGCTGTCATC-3' (-140 through -116); reverse: $5^{\prime}$-CCC ITCCTATGACATGAACTTAACCAT- $3^{\prime}(+566$ through +540$)$. The primer used for sequencing was: 5 '-CAGGTTGGTATCAAGGTTA-3' $(+139$ through +158$)$.

\section{DISCUSSION}

The variant, with a Leu->Pro substitution at position 31 of the $B$ chain, was described before as Hb Yokohama (1). Introduction of a proline residue at $B 31$ disrupts the heme globin contact, thus making the variant extremely unstable. This instability leads to the transfusion dependent hemolytic anemia. In this paper we present an observation of Hb Yokohama in a 10-year-old Yugoslavian boy. He exhibited moderately severe, transfusion-dependent hemolytic anemia, and was splenectomized at the age of 2.5 years. Since the parents of the boy were not carriers of this variant, the possibility of a de novo event was studied by evaluating the polymorphisms in the HLA DQ a locus. According to the genotype frequencies among Caucasians (14), the PI index of 1.97 and $W$ value of $66 \%$ were calculated (15). These results led us to consider this mutation as a de novo event.

Acknowledgements. This research was supported by the Yugoslav-American Joint Funds, pp 788 and Yugosiar Funds for Science and Technology, P-244GIBIT/SZNJ 1.5.1.5 ( $\mathrm{co}$ GDE), and by the Research Fund of the Republic of Croatia, No. 1.08.02.03.07 (to DJ).

\section{REFERENCES}

1. Nakatsuji, ... Hiwa, S., Ohba. Y.. Hattori, Y.., औiyaji, T., Hino. S., and Matsumoto, H. Hemoglobin, 5:507. 1981. 
2. Efremov, G.D., in Standardization of Laboratory Reagents and Methods for the Detection of Hemoglobinopathies, edited by R.M. Schmidt, T̈.H.J. Huisman, and H. Lehmann, page 135, CRC Press, Inc., Boca Raton, $\mathrm{FL}, 1974$.

3. Efremov, G.D., Markovska, B., Stojanovski, N., Petkov, G., Nikolov, N., and Huisman, T.H.J., Hemoglobin, 5:637, 1981.

4. Efremov, G.D., Huisman, T.H.J., Bowman, K., Wrightstone, R.N., and Schroeder, W.A., J. Lab. Clin. Med., 83:657, 1974.

5. Betke, K., Marti, H.R., and Schlicht, I., Nature, 184:1877, 1959.

6. Shelton, J.B., Shelton, J.R., and Schroeder, W.A., J. Liq. Chromatogr., 7:1969, 1984.

7. Huisman, T.H.J. and Jonxis, J.H.P., The Hemoglobinopathies Techniques of Identification, Clinical and Biochemical Analysis, Vol. 6, Marcel Dekker, Inc., New York, 1977.

8. Clegg, J.B., Naughton, M.A., and Weatheral1, D.J., J. Mol. Biol., $19: 91,1966$.

9. Wilson, J.B., Lam, H., Pravatmuang, P., and Huisman, T.H.J., J. Chromatogr., 179:271, 1979.

10. Schroeder, W.A., in The Hemoglobinopathies, edited by T.H.J. Huisman, Methods in Hematology, Vol. 15, page 142, Churchill Livingstone, Edinburgh, 1986.

11. Poncz, M., Solowiejczyk, D., Harpe1, B., Mory, Y., Schwartz, E., and Surrey, S., Hemoglobin, 6:27, 1982.

12. Saiki, R.K., Bugawan, T.L., Horn, G.T., Mullis, K.B., and Erlich, H.A., Nature, 324:163, 1986.

13. Sanger, F., Nicklen, S., and Coulson, A.R., Proc. Natl. Acad. Sci. USA, 74:5463, 1977.

14. AmpliTypeTM User Guide, Version 2, Cetus Corporation, 1990.

15. Sparkes, R.S. and Hodge, S.E., in Principles and Practice of Medical Genetics, edited by A.E.H. Emery and D.L. Rimoin, page 2011, Churchill Livingstone, Edinburgh, 1990. 


\title{
CHAPTER 5b
}

HB VOLGA [B 27(B9)ALA->ASP]: DETECTION OF A DE NOVO MUTATION BY AVA II DIGESTION OF PCR-AMPLIFIED DNA

\author{
D. Plaseska1, S. Koceva ${ }^{1}$, M. Rasovic ${ }^{2}$, and G.D. Efrenovl \\ 1 Research Center for New Technologies \\ Macedonian Academy of Sciences and Arts \\ 91000 Skopje, Republic of Macedonia \\ 2 Institute of Mother and Child Health Care \\ Clinic of Pediatrics, Faculty of Medicine \\ 11000 Belgrade, S.R. Yugoslavia
}

Adapted from: Hemoglobin, 17:209-215, 1993)

\section{ABSTRACT}

Hb Volga was observed as a de novo mutation in a 5-year-old boy from Tuzla, Bosnia and Hercegovina, who exhibited severe Heinz body hemolytic anemia. The variant was detected and quantitated at $10.6 \%$ by a reversed phase HPLC procedure. Structural characterization was done by HPLC analysis. An easier approach for the detection of Hb Volga by Ava II digestion of PCR-amplified DNA is described. 


\section{INTRODUCTION}

Hb Volga was first described in a Russian boy (1), and shortly after that the same abnormality was observed in a few members of a Dutch family (2). Since then it has been reported several times (3-6). We here describe a detection of $\mathrm{Hb}$ Volga as a de novo mutation in a young boy from Tuzla, Bosnia and Hercegovina. An easier approach for the detection of this variant by Ava II digestion of PCR-amplified DNA is given.

\section{MATERIALS AND METHODS}

Blood samples from the patient and his family were collected in vacutainers with EDTA as anticoagulant. Hematological data were obtained with an automated cell counter and routine hematological procedures. Red cell lysates were studied by starch gel electrophoresis at alkaline $\mathrm{pH}$ (7), and PAGE in the presence of urea, acid, and Triton X-100 (8). Heat stability of the $\mathrm{Hb}$ was tested at $52^{\circ} \mathrm{C}, 58^{\circ} \mathrm{C}$, and $62^{\circ} \mathrm{C}(9)$. The stability was also analyzed by isopropanol and PCMB tests (9). The percentage of $\mathrm{Hb}^{\mathrm{A}} \mathrm{A}_{2}$ and Hb $F$ was determined by microcolumn chromatography (10) and an alkali denaturation procedure (11), respectively. The abnormal $B$ chain was quantitated by reversed phase HPLC on a 1arge-pore $C_{4}$ column (12).

The abnormal Hb was isolated by precipitation with $17 \%$ isopropanol in $0.1 \mathrm{M} \mathrm{Tris} / \mathrm{HCl}, \mathrm{pH} 7.4$, at $37^{\circ} \mathrm{C}$ for 30 minutes. The abnormal $\mathrm{B}$ chain was separated from the a chain by CM-52 column chromatography (13) and digested with trypsin for 6 hours at room temperature and $\mathrm{pH} 8.5$. The tryptic peptides were separated by reversed phase HPLC on a $C_{18}$ column (14). The amino acid composition of the peptides was determined with a Beckman 6300 amino acid analyzer (Beckman, Palo Alto, CA, USA).

DNA was isolated from the leukocytes (15). A 706 bp fragment from the B-globin gene, including exon 1 and exon 2, was PCR-amplified (16). The primers used were $5^{\prime}$-6CCAAGGACAGGTACGGCTGTCATC- $3^{\prime}$ (direct) at positions -140 to -116 , and 5 '-CCCTTCCTATGACATGAACTTAACCAT-3' (reverse) at positions +566 to +540 , both relative to the Cap site of the $\beta-g l o b i n$ gene. The 706 bp amplified product was digested with Ava II for 4 hours at $37^{\circ} \mathrm{C}$, electrophoresed on $2.5 \%$ agarose gel, stained with ethydium bromide, and visualized under UV light.

The polymorphisms in the HLA DQa locus were evaluated with a Perkin Elmer Cetus (Norwalk, CT, USA) HLA DQ a Forensic DNA Amplification and Typing kit using the procedure recommended by the manufacturer.

\section{RESULTS AND DISCUSSION}

The propositus (J.E.) is a 5-year-old boy from Tuzla, Bosnia and Hercegovina. Anemia was noted when he was 3 months old. He was hospitalized in the clinic of Pediatrics in Belgrade in December 1990 because of high temperature, anemia, and subicterus. Physical examination revealed a normally developed boy with pale complexion. Cervical, supraclavicular, axillar, and ingvinal lymph nodes were palpable. The liver and spleen 
were palpable $3 \mathrm{~cm}$ below the costal margin. He received two blood transfusion while in hospital. A few weeks later he was referred to our institution for detailed investigation.

Hematological data for the propositus and his parents are given in Table $5 b / 1$. A peripheral blood smear from the propositus revealed anisocytosis, spherocytosis, and poikilocytosis. Heinz bodies were detectable after incubation of the blood at $37^{\circ} \mathrm{C}$ for 2 hours. The heat stability test at $52^{\circ} \mathrm{C}, 58^{\circ} \mathrm{C}$, and $62^{\circ} \mathrm{C}$ indicated the presence of $5-10 \%$ unstable $\mathrm{Hb}$. The isopropanol test was also positive. Starch gel electrophoresis of the precipitate obtained after PCMB treatment revealed the presence of ${ }_{\beta} A,{ }_{B} X$, and $\alpha A$ chains. No abnormal $H b$ was detected by starch gel electrophoresis and anion exchange chromatography. PAGE showed the presence of $a^{A}$ and $B A$ chains only. The abnormal $B$ chain was observed by reversed phase HPLC and estimated to represent $10.6 \%$ of the total Hb (Fig. 5b/1). The $\mathrm{Hb} \mathrm{A}_{2}$ and $\mathrm{Hb} F$ fractions amounted to 4.1 and $5.5 \%$, respectively. The parents had normal hematological and Hb findings.

TABLE 5b/1. Hematological Data

\begin{tabular}{lccccccccc}
\hline Subject & $\begin{array}{c}\mathrm{Hb} \\
\mathrm{g} / \mathrm{dl}\end{array}$ & $\begin{array}{c}\mathrm{PCV} \\
1 / 1\end{array}$ & $\begin{array}{c}\mathrm{RBC} \\
10^{12} / 1\end{array}$ & $\begin{array}{c}\mathrm{MCV} \\
\mathrm{fl}\end{array}$ & $\begin{array}{c}\mathrm{MCH} \\
\mathrm{pg}\end{array}$ & $\begin{array}{c}\text { Retics. } \\
(\%)\end{array}$ & $\begin{array}{c}\mathrm{A}_{2}^{\mathrm{a}} \\
\%\end{array}$ & $\begin{array}{c}\mathrm{Fb}^{\mathrm{b}} \\
\%\end{array}$ & $\begin{array}{c}\mathrm{X}^{\mathrm{C}} \\
\%\end{array}$ \\
\hline J.E. & 9.2 & .310 & 3.2 & 96.9 & 28.7 & 13.1 & 4.1 & 5.5 & 10.6 \\
Mother & 13.8 & .440 & 4.4 & 100.0 & 31.4 & n.d. & 2.5 & 0.9 & 0 \\
Father & 14.2 & .430 & 4.8 & 89.6 & 29.6 & n.d. & 2.5 & 0.3 & 0 \\
\hline
\end{tabular}

a By microcolumn chromatography (10).

b By alkali denaturation (11).

c By reversed phase HPLC (12).

n.d. = not determined.

Structural analysis of the ${ }_{\beta}{ }^{x}$ chain was done by reversed phase HPLC. The amino acid analysis of the abnomal tryptic peptides, eluting just in front of $B T-1$, showed the composition of the BT-3 peptide with the substitution of alanine by aspartic acid or asparagine. The only alanine in the normal $B T-3$ peptide is at position 27. A single base GCC->GAC substitution at that position would lead to an Ala->Asp replacement specific for the Hb Volga variant, while at least two base substitutions, namely GCC->AAC, would be required for an Ala->Asn replacement.

Since the GCC- $>$ GAC substitution creates a new Ava II restriction site, the $\mathrm{Hb}$ Volga gene can be detected by Ava II digestion. A 706 bp fragment (position -140 to position +566 , relative to the Cap site of the $B-$ globin gene) from the patient and a normal control was PCR-amplified and digested with Ava II. This fragment contains one constant Ava II site at position +293 and one polymorphic site at position +507 . An Ava II digest of the 706 bp fragment from the chromosome which lacks the polymorphic site would result in two fragments of 433 and $273 \mathrm{bp}$, while the fragment from the chromosome with the polymorphic site present would give three fragments of 433,214 , and $59 \mathrm{bp}$, respectively. If a GCC->GAC substitution at position +131 is present, a 433 bp fragment would be further divided into two smaller fragments of 271 and 162 bp. An Ava II restriction map of a 706 bp 


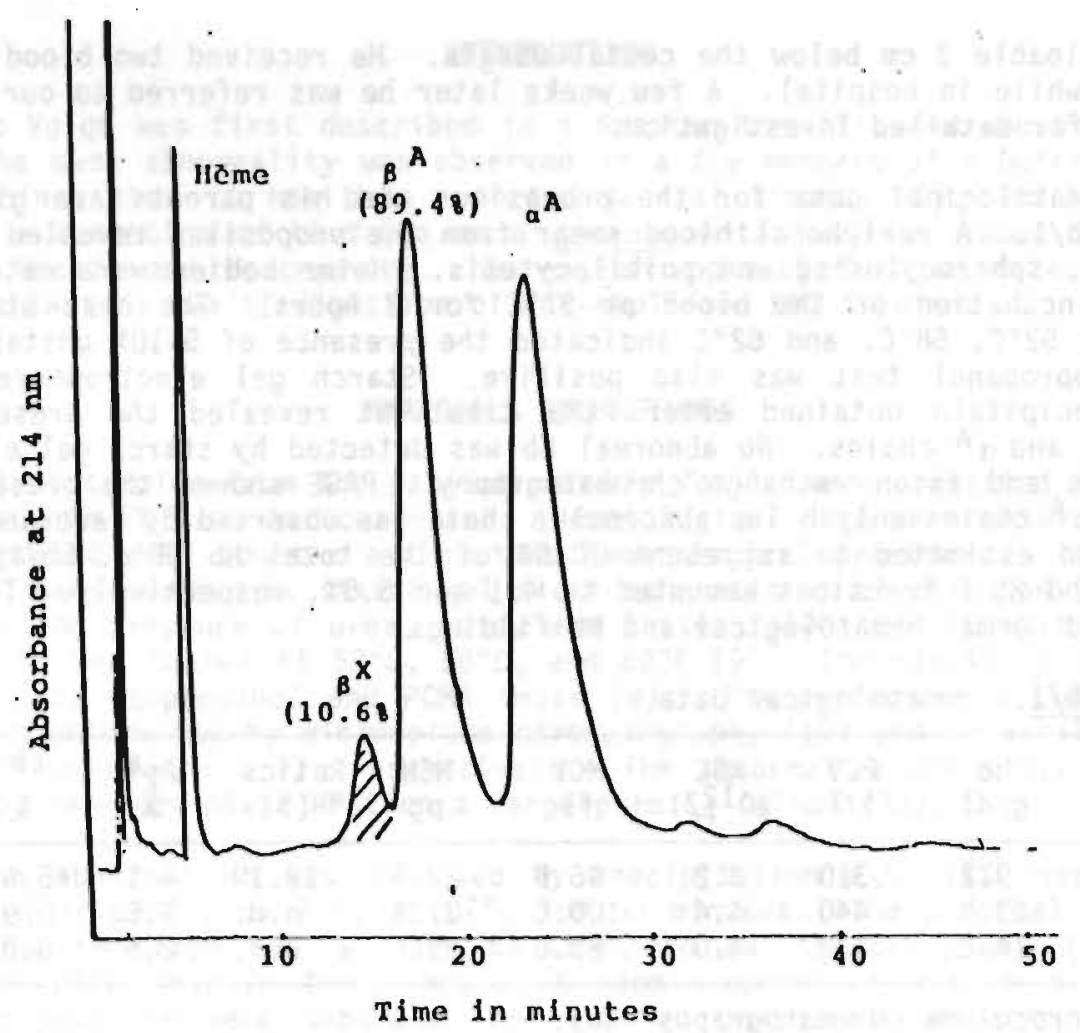

FIG. $5 b / 1$. Separation of $\beta^{X}, \beta^{A}$, and $a^{A}$ chains present in the red cell lysate of the propositus, by reversed phase HPLC.

PCR-amplified fragment from the normal chromosome and the chromosome with the $\mathrm{Hb}$ Volga mutation is shown in Fig. $5 \mathrm{~b} / 2$. Fragments of 433,273 , and 214 bp were obtained from the normal control, while four fragments of 433 , 271 (273), 214, and 162 bp were obtained from the patient. Only the 162 bp fragment is specific for $\mathrm{Hb} V$ Volga, since the $271 \mathrm{bp}$ fragment cannot be separated from the normal 273 bp fragment by agarose gel electrophoresis. The polymorphic site at position +507 is present on one chromosome only, both in the patient and in the normal control.

Since we could not detect this variant in the parents of the patient, we undertook paternity studies using the Perkin Elmer Cetus HLA DQa Forensic DNA Amplification and Typing kit. The results revealed genotype 1.2/2 for the mother, $4 / 4$ for the father, and 1.2/4 for the propositus. According to the genotype frequencies among Caucasians (17) the PI index of 25.6 and $W$ value of 96.6 were calculated (18), which made us consider this variant to have occurred as a de novo event.

In conclusion, we have described a fast, easy and new approach for detection of the Hb Volga mutation. The whole procedure can be performed in less than two days in laboratories that lack facilities for chemical analysis of proteins. We also described the first observation of $\mathrm{Hb}$ Volga in the Balkan peninsula. 


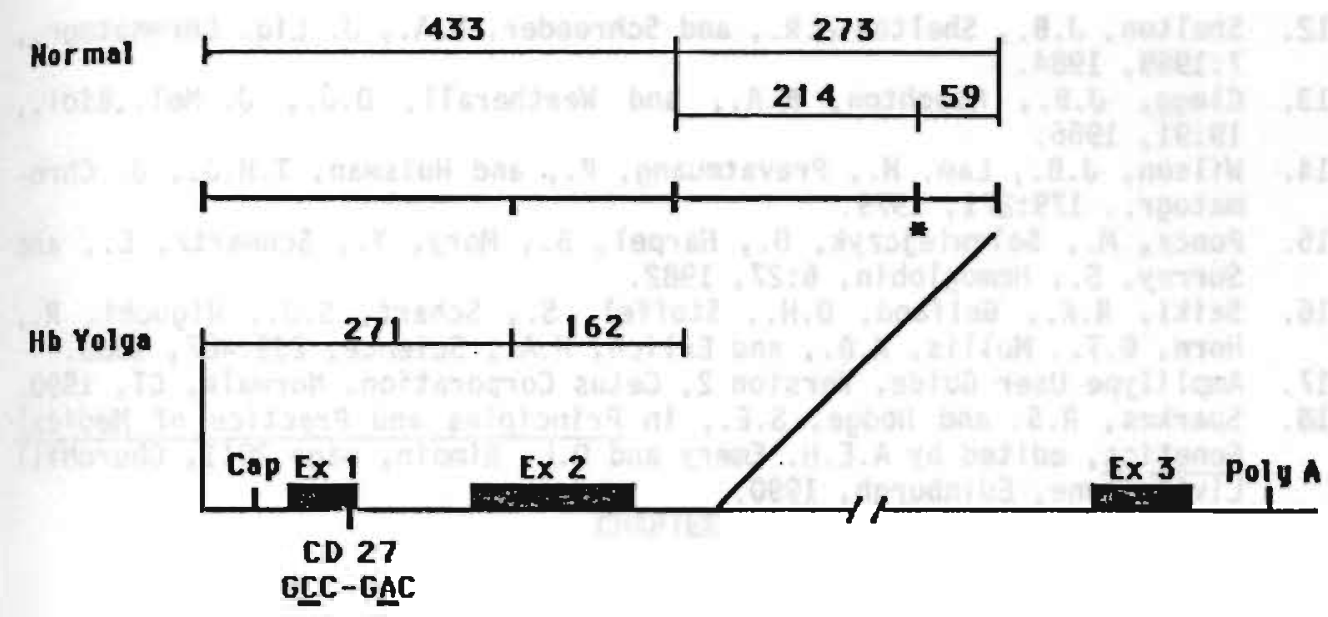

* Polymorphic site

FIG. 5b/2. An Ava II restriction map of the 706 bp fragment of the $\beta-g 10-$ bin gene from a patient with Hb Volga and a normal control.

Acknowledgements. This work was supported by the Ministry of Science of the Republic of Macedonia (336/92).

\section{REFERENCES}

1. Idelson, L.I., Didkovski, N.A., Filippova, A.V., Casey, R., Kynoch, P.A.M., and Lehmann, H., FEBS Lett., 58:122, 1975.

2. Kuis-Reeringk, J.D., Jonxis, J.H.P., Niazi, G.A., Wilson, J.B., Bolch, K.C., Gravely, M., and Huisman, T.H.J., Biochim. Biophys. Acta, 439: $63,1976$.

3. Brennan, S.0., Ekert, H., Tauro, G., and Carrell, R.W., N. Zealand Med. J., 84:162, 1976.

4. Ockelford, P.A., Liang, A.Y., Wells, R.M., Vissers, M., Brennan, S.0., Williamson, D., and Carrell, R.W., Hemoglobin, 4:295, 1980.

5. Sciarratta, G.V., Ivaldi, G., Sansone, G., Wilson, J.B., Webber, B.B., and Huisman, T.H.J., Hemoglobin, 9:91, 1985.

6. Blanke, S., Johnsen, A., and Wimberley, P.D., Hemoglobin, 13:489, 1989.

7. Efremov, G.D., in The Detection of Hemoglobinopathies, edited by R.M. Schmidt, T.H.J. Huisman, and H. Lehmann, page 7, CRC Press, Cleveland, $\mathrm{OH}, 1974$.

8. Efremov, G.D., Markovska, B., Stojanovski, N., Petkov, G., Nikolov, N., and Huisman, T.H.J., Hemoglobin, 5:637, 1981.

9. Huisman, T.H.J. and Jonxis, J.H.P., The Hemoglobinopathies Techniques of Identification, Clinical and Biochemical Analysis, Vol. $\overline{6}$, Marcel Dekker, Inc., New York, 1977.

10. Efremov, G.D., Huisman, T.H.J., Bowman, K., Wrightstone, R.N., and Schroeder, W.A., J. Lab. Clin. Med., 83:657, 1974.

11. Betke, K., Marti, H.R., and Schlicht, I., Nature, 184:1877, 1959. 
12. Shelton, J.B., Shelton J.R., and Schroeder, W.A., J. Liq. Chromatogr., $7: 1969,1984$.

13. Clegg, J.B., Naughton, M.A., and Weatheral1, D.J., J. Mol. Biol., $19: 91,1966$.

14. Wilson, J.B., Lam, H., Pravatmuang, P., and Huisman, T.H.J., J. Chromatogr., 179:271, 1979.

15. Poncz, M., Solowiejczyk, D., Harpel, B., Mory, Y., Schwartz, E., and Surrey, S., Hemoglobin, 6:27, 1982.

16. Saiki, R.K., Gelfand, D.H., Stoffel, S., Scharf, S.J., Higuchi, R., Horn, G.T., Mullis, K.B., and Erlich, H.A., Science, 239:487, 1988.

17. AmpliType User Guide, Version 2, Cetus Corporation, Norwalk, CT, 1990.

18. Sparkes, R.S. and Hodge, S.E., in Principles and Practice of Medical Genetics, edited by A.E.H. Emery and D.L. Rimoin, page 2011, Churchili Livingstone, Edinburgh, 1990. 


\title{
CHAPTER 6
}

\author{
Y CHAIN VARIANTS \\ I D E N T I F I E D B Y H P L C
}

6a Hb F-Jiangsu, the First $\gamma$ Chain Variant With a Valine->Methionine Substitution: ${ }_{\alpha 2}{ }^{A}{ }_{2} 134$ (H12) Yal->Met

6b Hb F-Catalonia or $\alpha_{2}{ }_{\mathrm{C}_{2}} 15$ (A12)Trp->Arg

6c Hb F-Brooklyn or $a_{2}{ }^{6} r_{2} 66$ (E10) Lys->61n 
7.

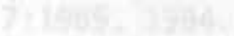

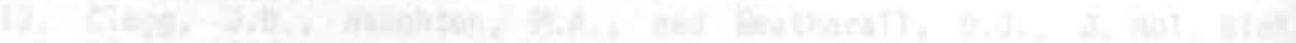

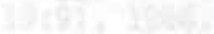

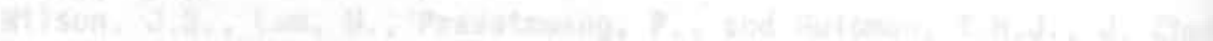

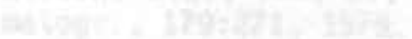

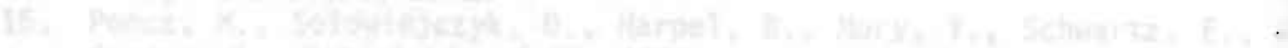

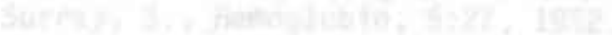

4.

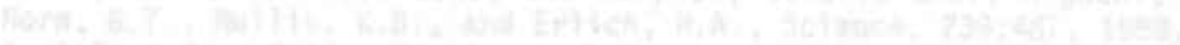

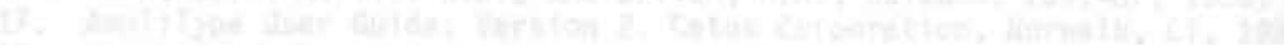

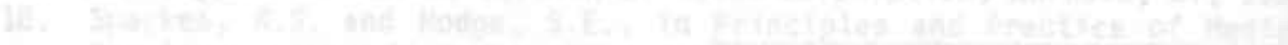

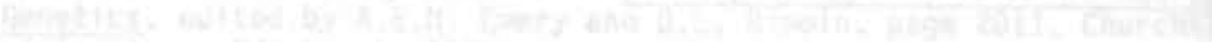

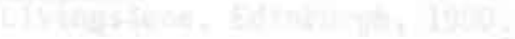

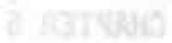




\title{
CHAPTER 6a
}

HB F-JIANGSU, THE FIRST $\gamma$ CHAIN VARIANT MITH A VALINE->METHIONINE SUBSTITUTION: $a 2^{A} \gamma_{2}^{134(H 12) \text { VAL->MET }}$

\author{
D. Plaseska1, F. Kutlarl, J.B. Milson', \\ B.B. Nebber1, Y-T. Zeng ${ }^{2}$, and T.H.J. Huisman'1 \\ 1 Department of Cell and Molecular Biology \\ Laboratory of Protein Chemistry \\ Medical College of Georgia, Augusta, GA 30912-2100, USA \\ 2 Laboratory of Medical Genetics \\ Shanghai Children's Hospital, Shanghai, P.R. China
}

(Adapted from: Hemoglobin, 14:177-183, 1990)

\section{ABSTRACT}

During a survey of cord blood samples of healthy Chinese babies an $A_{Y}$ variant was detected by $i$ ts partial separation from normal $A_{Y}$ in an HPL chromatographic experiment. Structural analysis of the isolated $A_{\gamma} X$ chain identified a Val->Met substitution at position $A_{\gamma 134 ;}$ this substitution created a rather unique Met-Met sequence. The ratio of $A_{\gamma} X$ to $A_{Y}$ was about 2 to 3 ; thus, $H \mathrm{~b} F$ with this variant $A_{\gamma}$ chain appears to have approximately normal stability. 


\section{INTRODUCTION}

Fifty-four $Y$ chain variants (1) have been detected through September 1989; 28 of these result from mutations in the $G_{\gamma-g l o b i n}$ gene and 17 from mutations in the $A_{\gamma-g l o b i n}$ gene; six have two substitutions including the Ile->Thr substitution at position $A_{\gamma} 75$, while for three variants the $A_{\gamma} 75$ information is not available. Institutions in the People's Republic of China have shown an increased interest in identifying $\gamma$ chain abnormalities and this led to the detection of $\mathrm{Hb}$ F-Urumq $\left[\mathrm{G}_{\gamma} 22(\mathrm{~B} 4) \mathrm{Asp}->\mathrm{Gl} \mathrm{y}\right]$, Hb F-Xinjiang $\left[A_{\gamma} T_{25}(B 7) G l y->A r g\right]$, Hb F-Shanghai [G $66(E 10)$ Lys->Arg], and Hb F-XinSu $\left[{ }^{A} 73(E 17) A s p->H i s\right] ;$ references are listed in Ref. 1.

In this communication we describe the fifth $\gamma$ chain variant observed among Chinese, named $\mathrm{Hb}$ F-Jiangsu. The substitution involves a valine- $>$ methionine at position 134 of the $A_{r}$ chain. This is the first mutation resulting in the introduction of a methionine residue; moreover, the -MetMet-sequence at positions $133-134$ is unique among the $\mathrm{Hb}$ polypeptide chains.

\section{METHODOLOGY}

The blood sample was shipped by air from Shanghaj to Augusta, GA. Collection was made in EDTA. Red cell lysates were studied by IEF (2) with commercially available agar plates (Isolab, Inc., Akron, OH, USA), and by reversed phase $\operatorname{HPLC}(3,4)$. Isolation of the normal and abnormal $A_{y}$ chains was by preparative reversed phase HPLC with a $30.0 \times 2.15 \mathrm{~cm} C_{18}$ column (5). Isolated chains were digested with trypsin for 6 hours at $\mathrm{pH} 8.5$ and at room temperature. The resulting peptides were separated by a reversed phase HPLC procedure described before (6). A few peptides were rechromatographed by a similar method but with water-acetonitrile-TFA developers (7). Amino acid analyses were made with the Waters Pico Tag amino acid analysis system and sequence analyses with a microsequencing procedure described by Chang et al (8).

\section{RESULTS AHD DISCUSSION}

The variant was detected unexpectedly when several cord blood samples from healthy babies of the Shanghaj area were analyzed by reversed phase HPLC to determine possible variations in the relative quantities of the $G_{Y}$ and $A_{\gamma}$ chains. Fig. $6 a / 1$ (top) shows the initial chromatogram indicating the possible presence of two different $A_{Y}$ chains. The ratio of $A_{Y} X$ and normal $A_{Y}$ was about 2 to 3 ; this ratio remained the same upon storage of the sample. The abnormal $Y$ chain $\left(A_{Y} X\right)$ was also separated from $G_{Y}$ and $A_{Y}$ by preparative reversed phase HPLC with a large $C_{18}$ column (Fig. $6 a / 1_{3}$ bottom). Several such chromatograms provided a few $\mathrm{mg}$ each of the $A_{\gamma}$ and the $A_{\gamma}$ chains for structural analyses.

Fig. 6a/2 illustrates the separation of the soluble peptides from tryptic digests of the two $A_{Y}$ chains by reversed phase HPLC methodology (6). The two chromatograms showed the presence of many minor zones which 

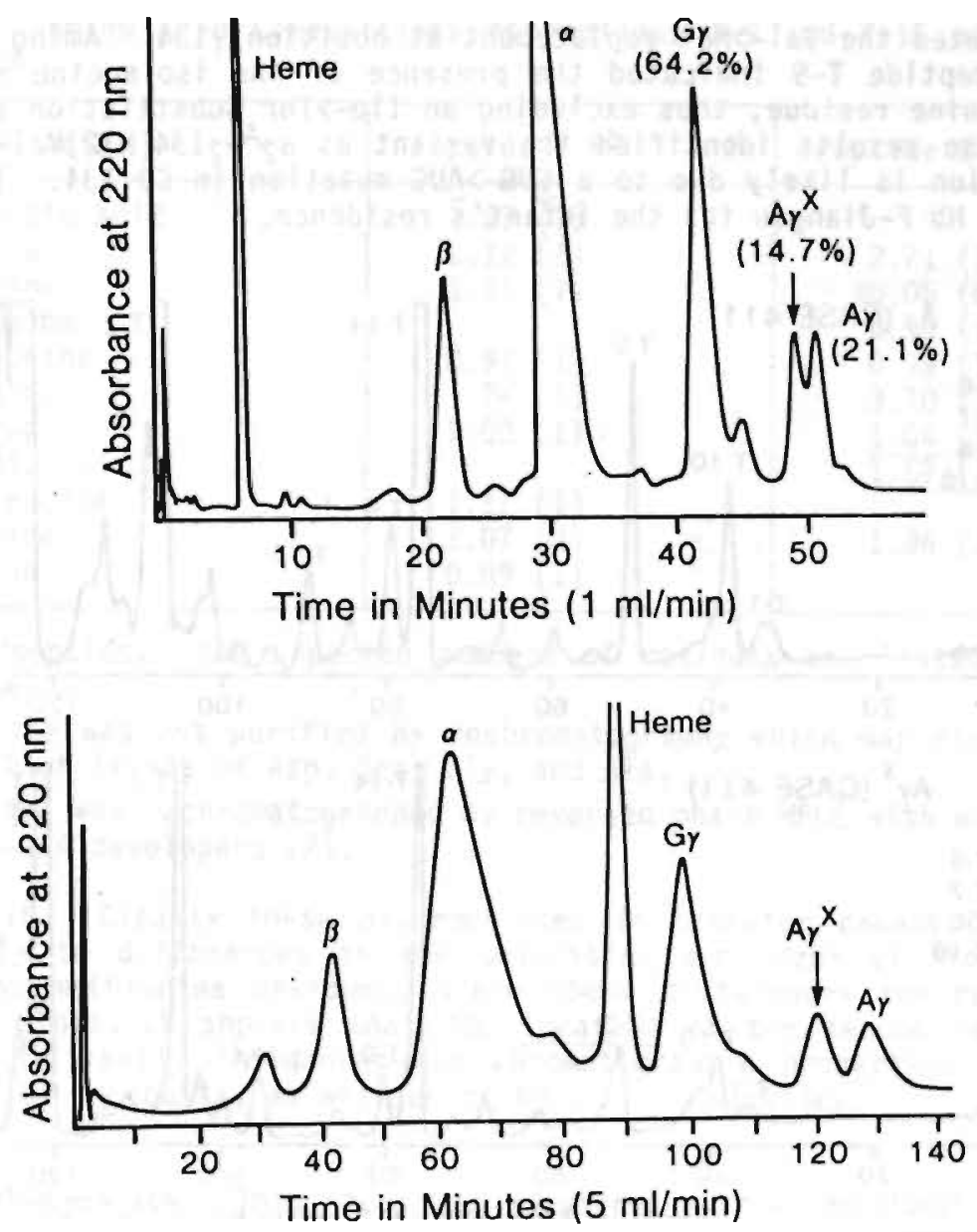

FIG. 6a/1. Separation of globin chains by reversed phase HPLC $(3,4)$. Top: An analytical chromatogram from the large-pore Vydac $C_{4}$ column. Bottom: A preparative chromatogram from a $C_{18}$ column (5). The amounts of $\mathrm{Hb}$ applied to the columns are estimated at 50 to $60 \mu \mathrm{g}$ (top) and 5 to $6 \mathrm{mg}$ (bottom).

are presumptive contaminants collected during the isolation of the small quantities of the two chains. However, the $r$ chain peptides $(T-1, T-2$, $T-3, T-4, T-5, T-6, T-7, T-8, T-9, T-10, T-14, T-15, T-16$ ) were readily recognized. All had the expected mobilities in this chromatographic system, except T-15 of the $A_{\gamma} X$ chain which eluted a few minutes later than the comparable peptide of the $A_{\gamma}$ chain. Peptide $T-15$ of $A_{\gamma} X^{X}$ was rechromatographed by reversed phase HPLC to obtain an acceptable analysis. Table $6 a / 1$ lists the amino acid composition of this peptide which indicates a Val->Met replacement. As peptide T-15 contains two valine residues, an additional quantity of this fragment was isolated and purified for sequence analysis. The result of that study gave: 
which located the Val->Met replacement at position $\gamma 134$. Amino acid analys 1 s of peptide T-9 indicated the presence of one isoleucine residue and one threonine residue, thus excluding an Ile->Thr substitution at position r75. These results identified the variant as $\alpha_{2} A_{\gamma_{2}} 134$ (H12)Val->Met; this substitution is likely due to a GUG->AUG mutation in CD 134. The variant was named $H b$ F-Jiangsu for the infant's residence.

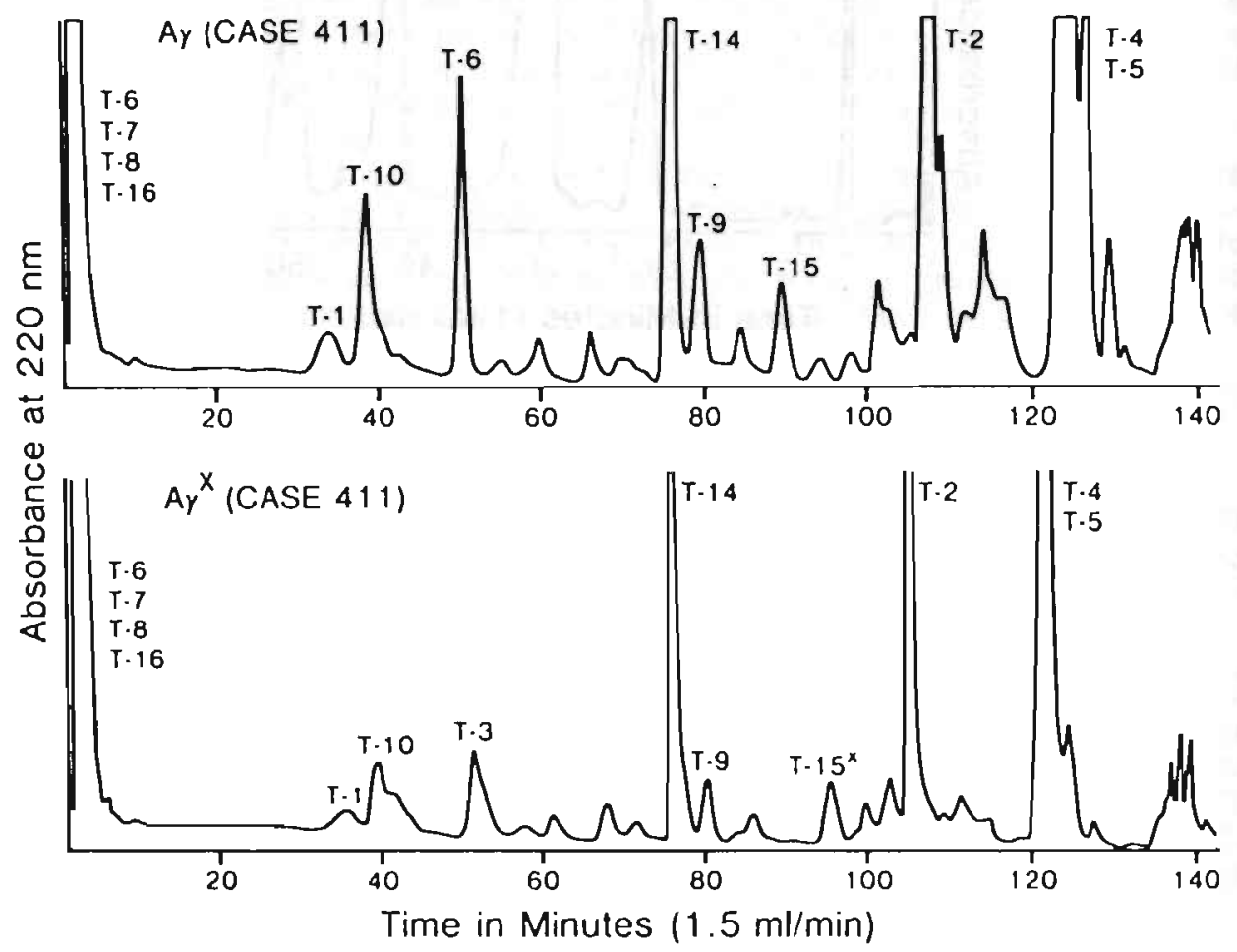

FIG. 6a/2. Separation of peptides present in the tryptic digests of the normal $A_{\gamma}$ and abnormal $A_{\gamma} X$ chains by a reversed phase HPLC method (6).

Because of the small volume of cord blood available, the low quantity of $\mathrm{Hb} F$-Jiangsu in this sample ( $\langle 20 \%)$, and the inability to separate $\mathrm{Hb} F$ Jiangsu from normal $\mathrm{Hb} F$, functional and physicochemical analyses were not conducted.

This Val->Met replacement is the first observed among the $\gamma$ chain variants. (See Ref. 1 for a comparison of the structures of the 54 abnormal $\gamma$ chains.) It is of some interest to compare its chromatographic behavior with that of the rather mysterious $M_{Y}$ chain which is characterized by a Leu->Met replacement in the same $Y T-15$ peptide but at position $\gamma 141$ $(9,10)$. The My chain $(10)$, al though it has alanine at residue 136 as does the $F$-Jiangsu $A_{\gamma}{ }^{X}$ chain, elutes before the $G_{\gamma}$ chain in the Vydac $C_{4}$ chromatographic system, and considerably earlier than the normal $A_{\gamma}$ and FJiangsu $A_{\gamma} X_{\text {chains. The }} M_{Y T-15}$ peptide also has a much shorter retention time than the normal $A_{Y T-15}$, but the F-Jiangsu $A_{Y T-15 X}$ elutes after the 
TABLE 6a/1. Amino Acid Compositions of Peptides T-9 and T-15 of the $A_{\gamma} X$ Chains a

\begin{tabular}{l|c|c}
\hline Amino Acid & Peptide T-gb & Peptide T-15c \\
\hline Aspartic acid & $1.59(1)$ & \\
Serine & $1.12(1)$ & $2.71(3)$ \\
Glycine & $1.25(1)$ & $0.05(0)$ \\
Arginine & $0.91(1)$ & $0.98(1)$ \\
Threonine & $1.24(1)$ & $3.99(1)$ \\
Alanine & $1.00(1)$ & $1.04(2)$ \\
Valine & $1.17(1)$ & $1.69(1)$ \\
Methionine & $2.07(2)$ & $1.06(1)$ \\
Isoleucine & $0.89(1)$ & \\
Leucine & Lysine & \\
\hline
\end{tabular}

a In moles/peptide. The expected numbers of residues are listed between parentheses.

b This peptide was not purified by rechromatography which may explain the somewhat high levels of Asp, Ser, Gly, and Ala.

c This peptide was rechromatographed by reversed phase HPLC with water-acetonitrile-TFA developers (7).

normal A $A_{\gamma}$ T-15. Clearly these discrepancies in behavior cannot be attributed solely to differences in the polarities and sizes of the valine, leucine, and methionine residues, since these differences are relatively minor (5). Thus, it appears that the location of the second methionine residue significantly influences the chromatographic properties of these three types of $A_{\gamma}$ chains, as well as of their T-15 peptides.

Acknowledgements. This study was supported in part by USPHS research grants HLB-05168 and HLB-41544. This is contribution \#1212 from the Department of Cell and Molecular Biology at the Medical College of Georgia in Augusta, GA.

\section{REFERENCES}

1. International Hemoglobin Information Center, Hemoglobin, 13:223, 1989.

2. Righetti, P.G., Gianazza, E., Bianchi-Bosisio, A., and Cossu, G., in The Hemoglobinopathies, edited by T.H.J. Huisman, Methods in Hematology, Vol. 15, page 47, Churchill Livingstone, Edinburgh, 1986.

3. Shelton, J.B., Shelton, J.R., and Schroeder, W.A., J. Liq. Chromatogr., 7:1969, 1984.

4. Kutlar, F., Kutlar, A., and Huisman, T.H.J., J. Chromatogr., 357:147, 1986.

5. Huisman, T.H.J., J. Chromatogr., 418:277, 1987.

6. Wilson, J.B., Lam, H., Pravatmuang, P., and Huisman, T.H.J., J. Chromatogr., 179:271, 1979. 
7. Schroeder, W.A., in The Hemoglobinopathies, edited by T.H.J. Huisman, Methods in Hematology, Vol. 15, page 142, Churchill Livingstone, Edinburgh, 1986.

8. Chang, J.Y., Brauer, D., and Wittmann-Liebold, B., FEBS Lett., 93:205, 1978.

9. Huisman, T.H.J., Kutlar, F., Kutlar, A., Wilson, J.B., and Harris, H.F., in Progress in Clinical and Biological Research, edited by G. Stamatoyannopoulos and A.W. Nienhuis, Developmental Control of Globin Gene Expression, Vol. 251, page 507, Alan R. Liss, Inc., New York, 1987.

10. Huisman, T.H.J., Kutlar, F., Kutlar, A., Wilson, J.B., and Harris, H.F., J. Chromatogr., 388:429, 1987. 


\section{CHAPTER 6b}

HB F-CATALONIA OR $\alpha_{2}{ }^{G}{ }_{\gamma_{2}} 15$ (A12)TRP->ARG

D. Plaseskal, J.B. Wilson 1, F. Kutlar ${ }^{1}$,

L1. Font ${ }^{2}$, M. Baiget ${ }^{3}$, and T.H.J. Huisman 1

1 Department of Cell and Molecular Biology

Medical College of Georgia, Augusta, GA 30912-2100, USA

2 Servei d'Hematologia, Hospital Verge de la Cinta, Tortosa, Spain

3 Molecular Genetics Unit, Hospital Santa Creu i Sant Pau 08025 Barcelona, Spain

(Adapted from: Hemoglobin, 14:511-516, 1990)

\section{ABSTRACT}

Hb F-Catalonia, a $G_{Y}$ chain variant with a Trp->Arg substitution at position $\gamma 15(A 12)$, was observed in two Spanish newborn babies from Northeastern Spain. Analyses were performed with different reversed phase HPLC procedures that allowed the identification of the amino acid replacement in only a minute quantity of the isolated $G_{\gamma} X$ chain. 


\section{INTRODUCTION}

The testing of newborn babies for the presence of $\mathrm{Hb}$ abnormalities has become popular worldwide; it has also resulted in the detection of numerous $r$ chain variants (see Ref. 1 for a list and references). A new variant was recently detected during a survey of newborn babies from the northeastern part of Spain. The abnormality was present in two infants living in Tortosa, Tarragona Province, which is located in the delta of the Ebro River; its characterization is described here.

\section{MATERIALS AND METHODS}

Blood samples were collected with EDTA as anticoagulant and studied at the laboratory of the local institution in Barcelona, while part was shipped (as frozen washed red cellis) by air to Augusta, GA. Informed consent from the parents was obtained.

Red cell lysates were studied by $\operatorname{IEF}(2)$, cation exchange $\operatorname{HPLC}(3,4)$, and by reversed phase HPLC $(5,6)$. A larger quantity of the variant was isolated by DEAE-cellulose chromatography (7), while the a and $\gamma^{X}$ chains of the isolated $\mathrm{Hb} \mathrm{FX}_{X}$ were separated by reversed phase HPLC using a preparative $C_{18}$ column $(\hat{B})$. The relative quantity of the $\gamma^{X}$ chain (as $\%$ of total $\gamma$ ) was determined by reversed phase HPLC using a large-pore Vydac $\iota_{4}$ column $(5,6)$.

The $\gamma^{X}$ chain was digested with trypsin for 6 hours at room temperature and at pH 8.2. The tryptic peptides were separated by reversed phase HPLC (9) and their amino acid compositions determined with a Waters Pico Tag automated amino acid analyzer.

\section{RESULTS AND DISCUSSION}

The two babies had identical Hb patterns in different electrophoretic and chromatographic experiments. Structural analyses of the $\gamma x$ cliains from both infants showed that the substitution was the same; data for the variant present in the larger sample are presented here.

Detection of the abnormal $\mathrm{Hb}$ was by cellulose acetate electrophoresis and IEF; it had a mobility between those of $\mathrm{HbS} S$ and $\mathrm{C}$ in both systems. The parents of the infants did not have the abnormality. It was readily isolated on a column of DEAE-cellulose. Globin chain analysis on a reversed phase HPLC system showed that the $\gamma^{X}$ chain eluted slightly faster than the normal $G_{\gamma}$ chain (Fig. 6b/1A). The $\gamma^{X}$ and $G_{\gamma}$ chains together accounted for $72.2 \%$; this normal value was considered to indicate that ${ }^{X}$ was a mutant of the $G_{Y}$ chain. The pattern for the $H b$ in the red cell iysate of the second baby was the same except for an additional $A_{Y} T$ heterozygosity; the four $Y$ chains, $A_{Y} T, G_{\gamma} X, G_{Y}$, and $A_{Y}$, were eluted in that order and were present for $11.5 \%, 45.5 \%, 31.3 \%$, and $11.7 \%$, respectively (the higher $G_{Y} X$ value is likely due to an incomplete separation of $A_{Y}^{\top}$, $G_{Y} X$, and $\left.G_{Y}\right)$. Isolated $H b F_{X}$ was nearly pure and contained a and $G_{Y} X$ 
chains only (Fig. 6b/lB). As only a small quantity of Hb $F_{X}$ was available, the $a$ and $G_{\gamma} X$ chains were separated by preparative reversed phase HPLC rather than by the common CM-cellulose chromatographic procedure; the separation of $a$ and $G_{r} X$ was partially complete (Fig. $6 \mathrm{~b} / \mathrm{lC}$ ).
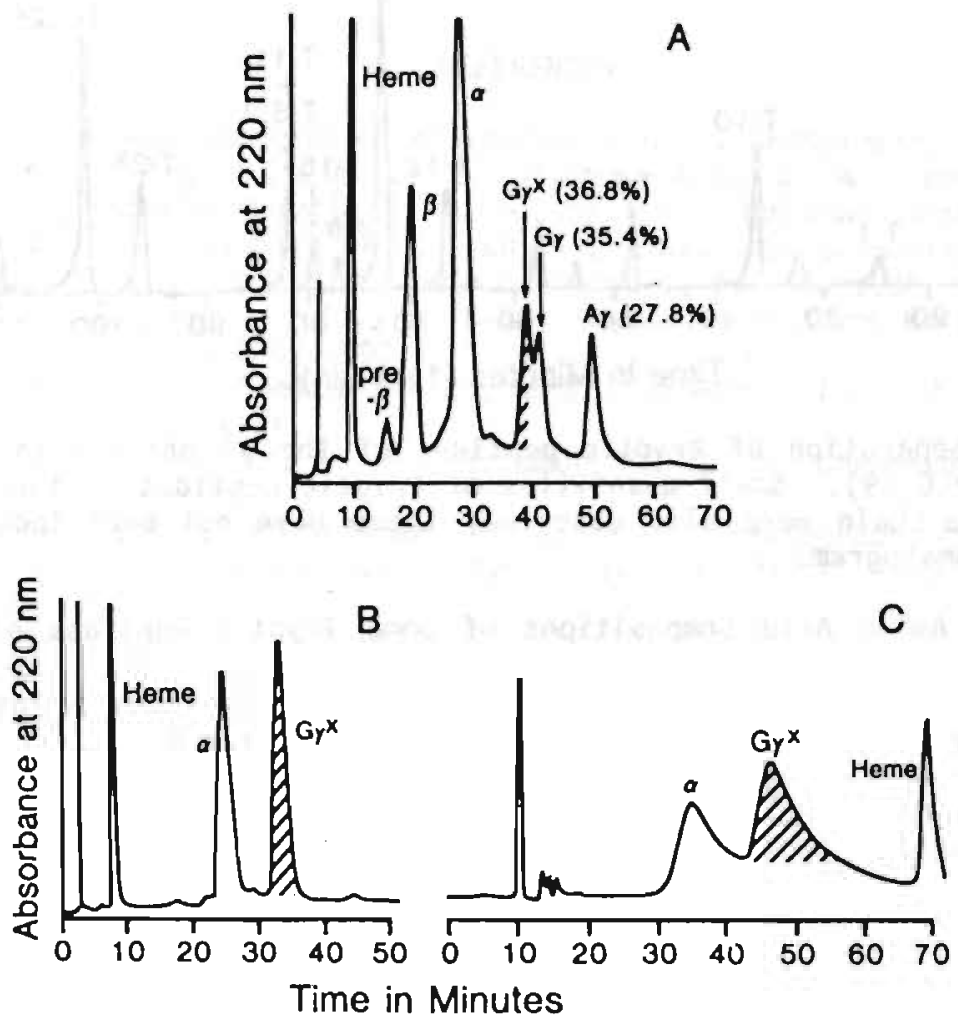

FIG. 6b/1. Separation of the globin chains by reversed phase HPLC $(5,6)$. $A$ : Whole red cell lysate; $B$ and $C$ : $H b F_{X}$, isolated by DEAE-cellulose chromatography. Chromatograms A and B were developed with an analytical large-pore Vydac $C_{4}$ column, and chromatogram $C$ with a preparative $\mathrm{C}_{18}$ column.

Fig. 6b/2 illustrates the separation of the peptides present in a tryptic digest of the $G_{\gamma} X$ chain; the partial separation of $\alpha$ and $G_{\gamma} X$ on the reversed phase $C_{18}$ column ( $F$ ig. $6 \mathrm{~b} / \mathrm{lC}$ ) was responsible for the presence of notable quantities of $\alpha$ chain peptides. All soluble $\gamma$ chain peptides were recovered and identified. Their amino acid compositions were normal except for those of peptides $T-2^{X}$ and $T-1,{ }_{2}{ }^{X}$ which showed an extra arginine residue that replaced the normally occurring tryptophan (Table $6 \mathrm{~b} / 1)$. This substitution occurred at position $\gamma 15$ and $H b F_{X}$, therefore, has as structure $a_{2} \gamma_{2}$ 15(A12)Trp->Arg. The data obtained for peptides $T-8,9$ and $T-15$ (Table $6 \mathrm{~b} / 1$ ) confirmed the suspicion that the $\gamma^{X}$ chain was a variant of the $G_{Y}$ chain (peptide $T-15$ has one glycine and two alanine residues; peptide T-8,9 had one isoleucine and one threonine residue). 


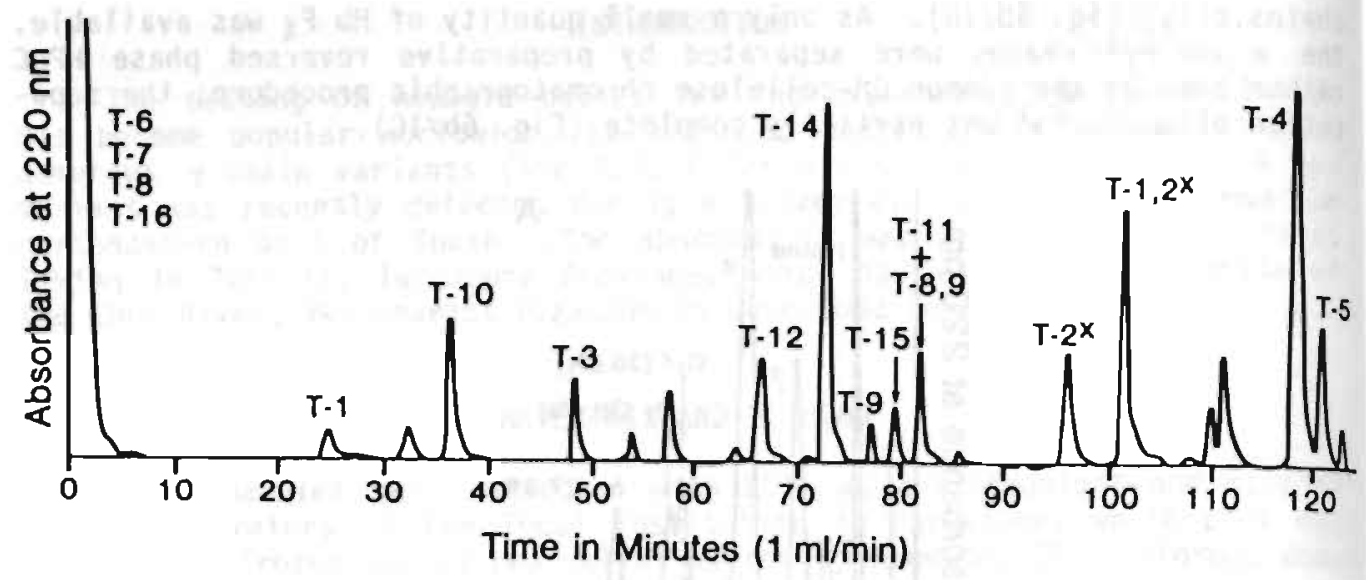

FIG. 6b/2. Separation of tryptic peptides of the $\gamma^{X}$ chain with reversed phase HPLC (9). Small quantities of tryptic peptides of the contaminating a chain were also observed; these have not been indicated in the chromatogram.

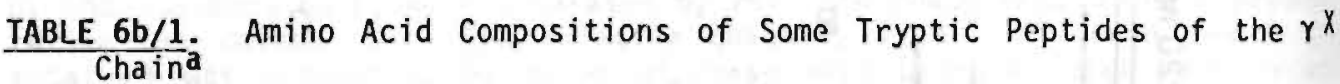

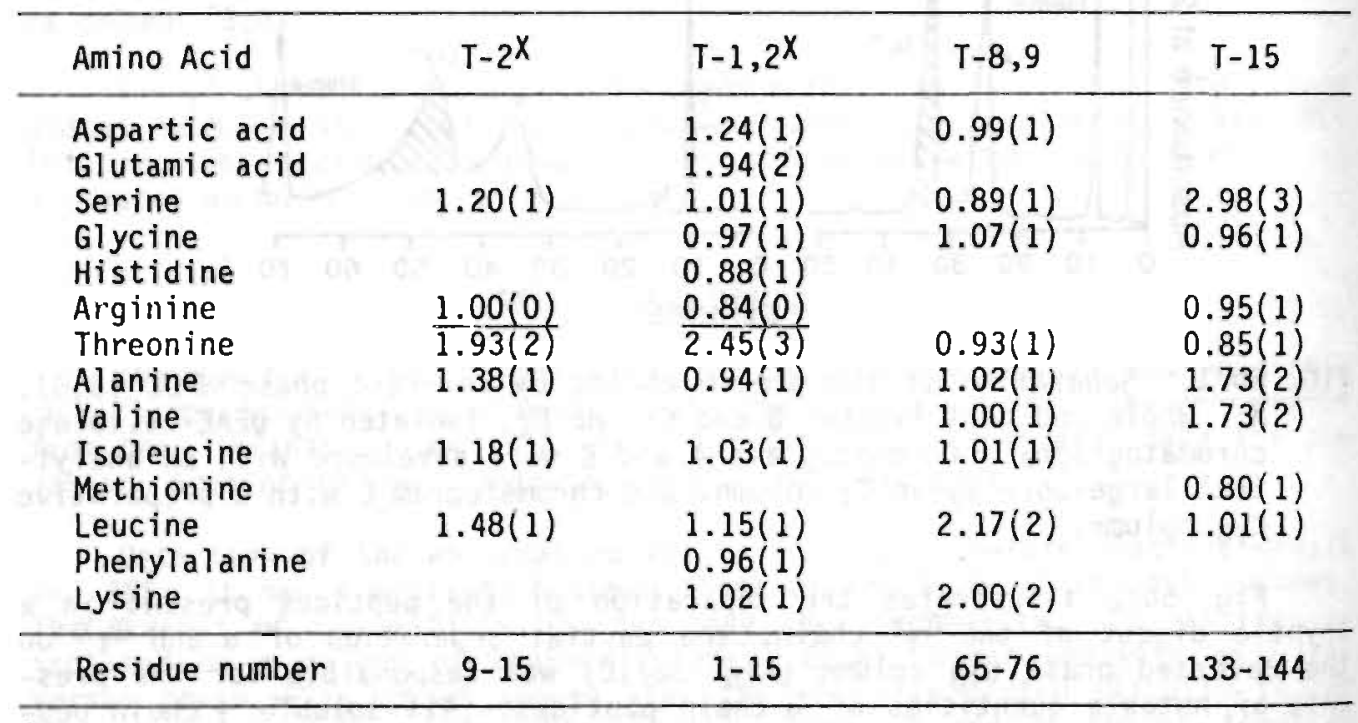

a In moles/mole; numbers between parentheses are those expected for the tryptic peptides of the normal $G_{\gamma}$ chain.

The Hb FX has been named F-Catalonia for the area where it was discovered. It is unknown if the $\gamma 15$ Trp->Arg substitution has any effect on the functional or physicochemical properties of the fetal $\mathrm{Hb}$; the studies required to determine this were not possible due to a complete lack of material. 
Acknowledgements. This study was in part supported by USPHS research grant HLB-05168 (to THJH). This is contribution $\# 1250$ from the Department of Cell and Molecular Biology at the Medical College of Georgia in Augusta, GA.

\section{REFERENCES}

1. International Hemoglobin Information Center, Hemoglobin. 14:249, 1990.

2. Righetti, P.G., Gianazza, E., Bianchi-Bosisio, A., and Cossu, G., in The Hemoglobinopathies, edited by T.H.J. Huisman, Methods in Hematology, Vol. 15, page 47, Churchill Livingstone, Edinburgh, 1986.

3. Bisse, E. and Wieland, H., J. Chromatogr., 434:95, 1988.

4. Kutlar, A., Kutlar, F., Gu, L-G., Mayson, S.M., and Huisman, T.H.J., Hum. Genet., 85:106, 1990.

5. Shelton, J.B., Shelton, J.R., and Schroeder, H.A., J. Liq. Chromatogr., 7:1969, 1984.

6. Kutlar, F., Kutlar, A., and Huisman, T.H.J., J. Chromatogr., 357:147, 1986 .

7. Schroeder, W.A. and Huisman, T.H.J., The Chromatography of Hemoglobin, Clinical and Biochemical Analysis, Vol. 9, Marcel Dekker, Inc., New York, 1980.

8. Huisman, T.H.J., J. Chromatogr., 418:277, 1987.

9. Wilson, J.B., Lam, H., Pravatmuang, P., and Huisman, T.H.J., J. Chromatogr., 179:271, 1979. 


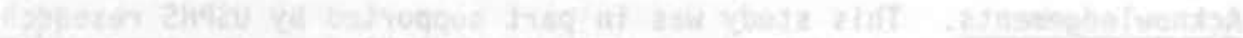

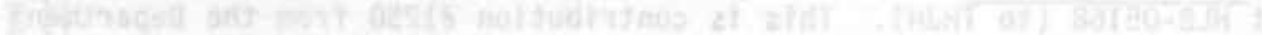

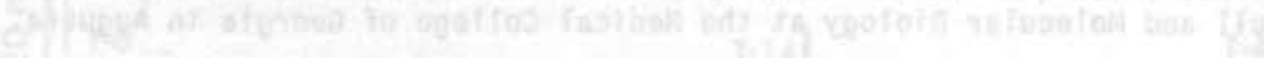

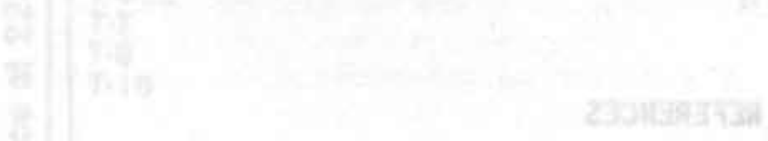

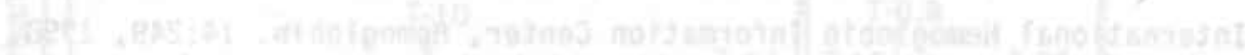

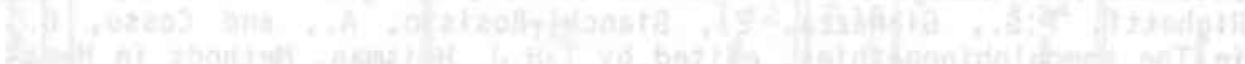

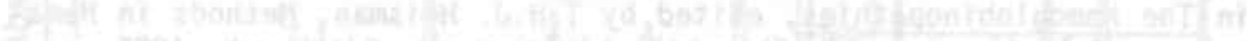

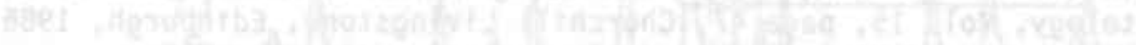

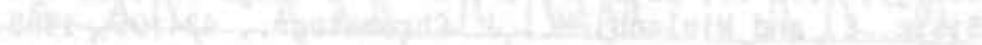

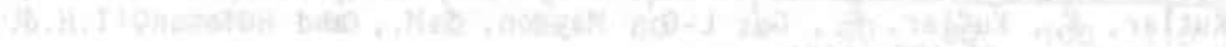
(1)

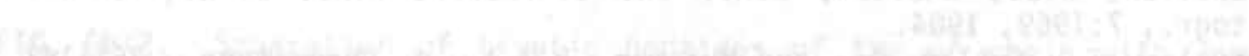

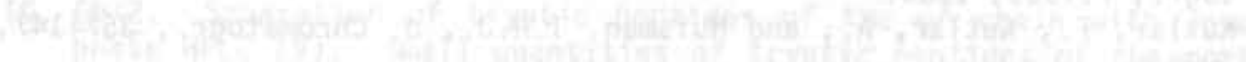

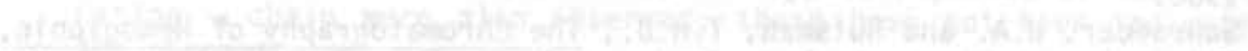

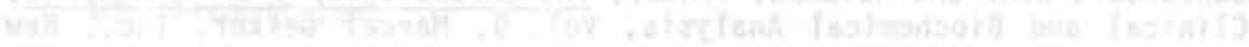

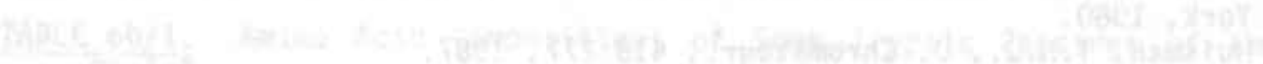

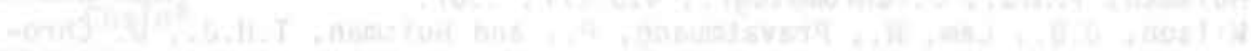

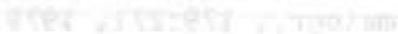

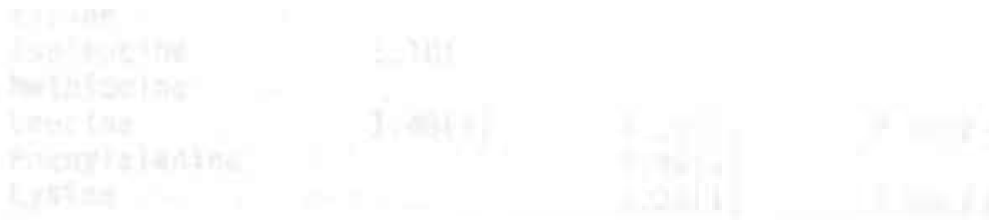




\section{CHAPTER 6c}

\section{HB F-BROOKLYN OR $\alpha_{2}{ }^{G}{ }_{2} 66$ (E10)LYS->GLN}

D. Plaseskal, H. Li1, J.B. Wilson 1, F. Kutlarl,

A. Kutlarl, T.H.J. Huismanl, and J. Kulpa ${ }^{2}$

1 Laboratory of Protein Chemistry

Department of Cell and Molecular Biology

Medical College of Georgia, Augusta, GA 30912-2100, USA

2 The Long Island College Hospital, Brooklyn, N.Y. 11201, USA

(Adapted from: Hemoglobin, 14:213-216, 1990)

Routine screening of blood samples from newborn babies or infants for $\mathrm{Hb}$ abnormalities often results in the discovery of (minor) abnormal $\mathrm{Hb}$ components which are difficult to characterize but are most frequently $\gamma$ chain variants. This was true for a 2-month-old Caucasian female; her red cell lysate exhibited a minor, fast-moving, variant when examined by alkaline electrophoresis. About $5 \mathrm{ml}$ blood containing $500 \mathrm{mg} \mathrm{Hb}$ was available for further analysis.

The variant was detectable by IEF (1) and by cation exchange HPLC (2); quantitation by the latter procedure estimated $\mathrm{Hb} \mathrm{FX}_{X}$ to be about $23 \%$ of the total $\mathrm{Hb}$. The best separation was seen in reversed phase HPLC $(3,4)$. The abnormal $\gamma$ chain $\left(G_{\gamma} X\right)$ eluted between the normal $G_{\gamma}$ and $A_{\gamma}$ chains (Fig. $6 \mathrm{c} / 1)$; its quantity was $25.1 \%$ of the total $\gamma$ chains which corresponds to $17.4 \%$ of the total $\mathrm{Hb}$. Preliminary identification of the abnormal chain as a variant $G_{\gamma}$ chain is based on the ratio of $\left(G_{\gamma}+G_{\gamma} X\right)$ to $A_{\gamma}$ of 64.4 to $35.6 \%$, which is normal for most newborn babies.

The variant Hb was isolated by DEAE-cellulose chromatography (5) and the $G_{\gamma} X$ and a chains were separated by preparative reversed phase HPLC (6). Tryptic digests of the $G_{\gamma} X$ chain was at room temperature and at $\mathrm{pH}$ 8.5 for a period of 6 hours. When the soluble tryptic fragments were separated by a reversed phase HPLC procedure (7), all except the T-9 peptide had their expected positions in the chromatogram. Peptide T-9 did not appear in its normal position between T-14 and T-15, but a shoulder was observed on the early side of T-15. Rechromatography with a slightly different HPLC procedure (8) separated the two peptides; their amino acid compositions are presented in Table $6 \mathrm{c} / 1$. One was a normal $\mathrm{G}_{\gamma} T-15$ and the other was T-9 plus glutamic acid or glutamine. A manual sequencing 
procedure (9) showed the latter peptide to contain at its amino terminus -Gln-Val-Leu. Therefore, lysine at position $\gamma 66$ is replaced by glutamine. This conclusion is further supported by the absence of free lysine ( $r T-8$ ) from the front zone of the chromatogram. These results identify a Lys $>$ Gln substitution at position $\gamma 66$. The variant was named $H b$ F-Brooklyn after the patient's residence. The amino acid composition of $r \mathrm{~T}-15$ identified the presence of one glycine residue and two alanine residues, thus confirming that $\mathrm{Hb}$ F-Brooklyn is a Grvariant.

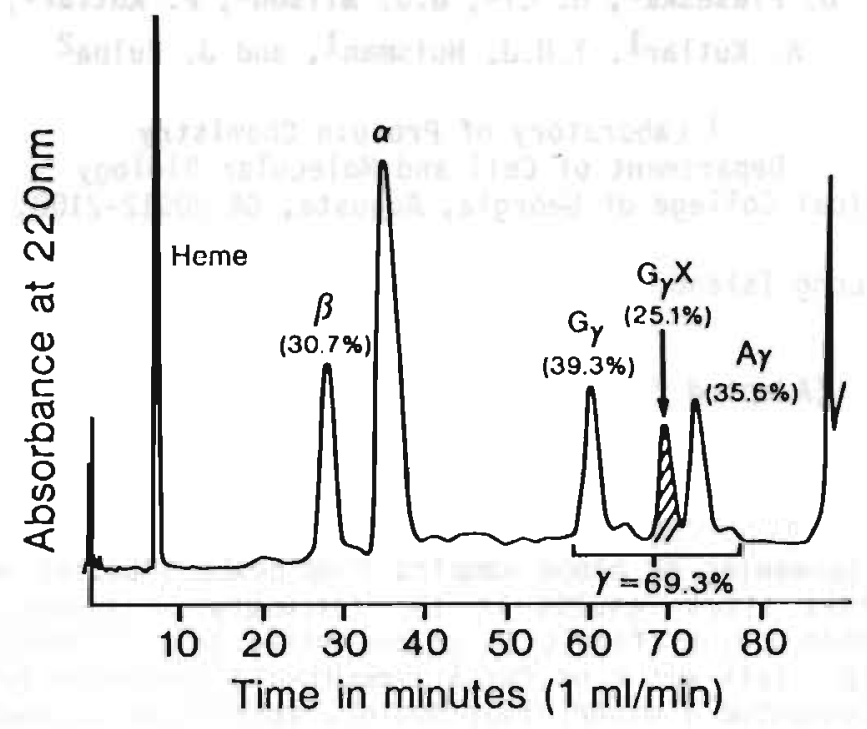

FIG. $6 c / 1$. Separation of globin chains in a red cell lysate of the baby with $\mathrm{Hb} F-B$ rooklyn or ${ }^{\alpha_{2}} \mathrm{G}_{\gamma_{2}} 66(E 10)$ Lys->Gln by a reversed phase HPLC procedure.

TABLE 6c/1. Amino Acid Composition of Two Peptides (Gy-Brooklyn) ${ }^{a}$

\begin{tabular}{l|c|c}
\hline Amino Acid & T-8,9 & T-15 \\
\hline Aspartic Acid & $1.04(1)$ & \\
Threonine & $0.97(1)$ & $0.91(1)$ \\
Serine & $0.97(1)$ & $2.53(3)$ \\
Glutamic Acid & $1.12(0)$ & $1.00(1)$ \\
Glycine & $1.08(1)$ & $1.98(2)$ \\
Alanine & $1.11(1)$ & $1.87(2)$ \\
Valine & $0.99(1)$ & $0.80(1)$ \\
Methionine & $1.99(2)$ & $0.96(1)$ \\
Leucine & $0.92(1)$ & \\
Isoleucine & $0.93(2)$ & $1.00(1)$ \\
Lysine & & \\
Arginine & & \\
\hline
\end{tabular}

a In moles/peptide; the numbers between parentheses represent the expected number of residues for the normal peptide. 
Hb F-Brooklyn is the second $G_{\gamma}$ chain variant in which lysine at posi-

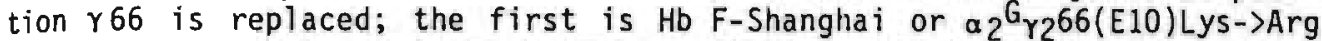
(10). Two $B$ chain variants have been found in which lysine at the same position is replaced by glutamic acid in $\mathrm{Hb}$ I-Toulouse (11) and by threonine in Hb Chico (12). The instability of Hb I-Toulouse is likely caused by the loss of the nonpolar "plug" which normally seals the protein surface; the substitution to glutamic acid allows water to enter the heme crevice $(13,14)$. Hb Chico is less unstable and does not cause an overt hemolytic disorder. Similar data for both Hb F-Shanghai [GyG6(E10)Lys-> Arg] and Hb F-Brooklyn [GYG6(E10)Lys->Glnj are not available as material for detailed stability analyses was not available.

Acknowledgements. This research was supported by USPHS research grant HLB-05168. This is contribution \#1216 from the Department of Cell and Molecular Biology at the Medical College of Georgia in Augusta, GA.

\section{REFERENCES}

1. Righetti, P.G., Gianazza, E., Bianchi-Bosisio, A., and Cossu, G., in The Hemoglobinopathies, edited by T.H.J. Huisman, Methods in Hematology, Vol. 15, page 47, Churchill Livingstone, Edinburgh, 1986.

2. Wilson, J.B., Headlee, M.E., and Huisman, T.H.J., J. Lab. Clin. Med., $102: 174,1983$.

3. Shelton, J.B., Shelton, J.R., and Schroeder, W.A., J. Liq. Chromatogr., 7:1969, 1984.

4. Kutlar, F., Kutlar, A., and Huisman, T.H.J., J. Chromatogr., 357:147, 1986.

5. Schroeder, W.A. and Huisman, T.H.J., The Chromatography of Hemoglobin, Clinical and Biochemical Analysis, Vol. 9, Marcel Dekker, Inc., New York, 1980.

6. Huisman, T.H.J., J. Chromatogr., 418:277, 1987.

7. Wilson, J.B., Lam, H., Pravatmuang, P., and Huisman, T.H.J., J. Chromatogr., 179:271, 1979 .

8. Schroeder, W.A., in The Hemoglobinopathies, edited by T.H.J. Huisman, Methods in Hematology, Vol. 15, page 142, Churchill Livingstone, Edinburgh, 1986.

9. Chang, J.Y., Brauer, D., and Wittmann-Liebold, B., FEBS Lett., 93:205, 1978.

10. Zeng, Y.T., Huang, S.Z., Nakatsuji, T., and Huisman, T.H.J., Am. J. Hematol., 18:235, 1985.

11. Rosa, J., Labie, D., Wajcman, H., Boigne, J.M., Cabannes, R., Bierme, R., and Ruffie, J., Nature, 223:190, 1969.

12. Shih, D.T-b., Jones, R.T., Shih, M.F-C., Jones, M.B., Koler, R.D., and Howard, J., Hemoglobin, 11:453, 1987.

13. Bunn, H.F. and Forget, B.G., Hemoglobin: Molecular, Genetic and Clinical Aspects, H.B. Saunders Company, Philadelphia, PA, 1986.

13. Ohba, Y., Hemoglobin, 14:353, 1990. 


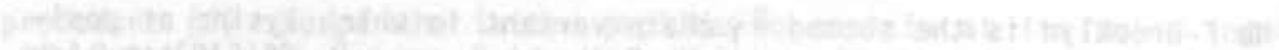

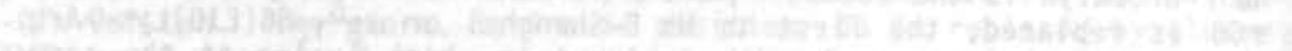

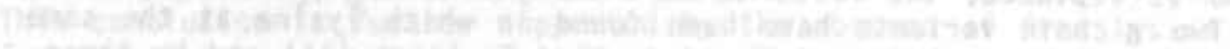

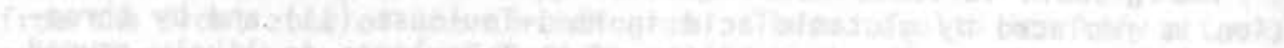

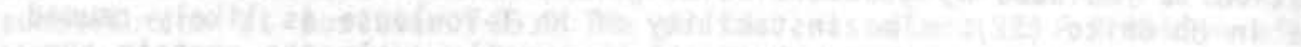

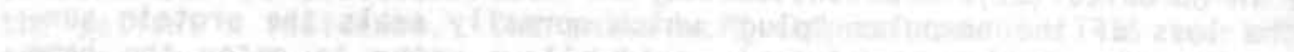

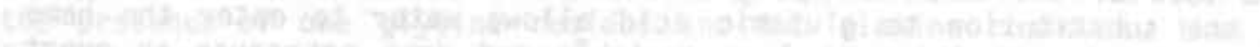

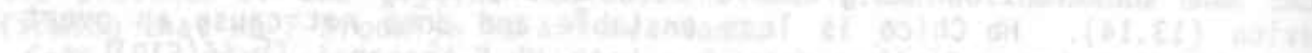

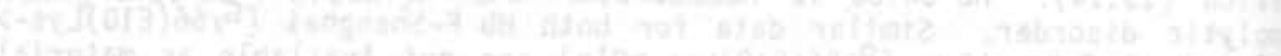

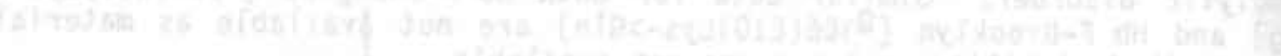

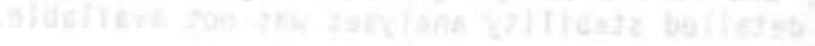

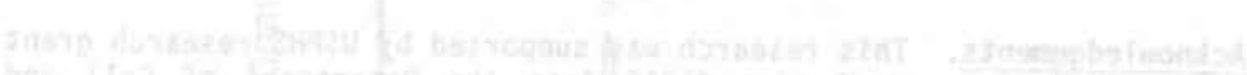

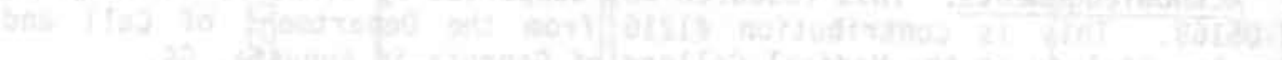

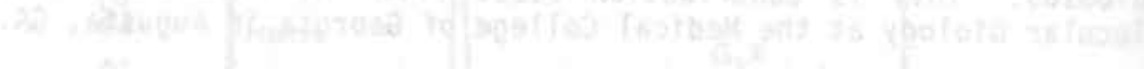

[8.

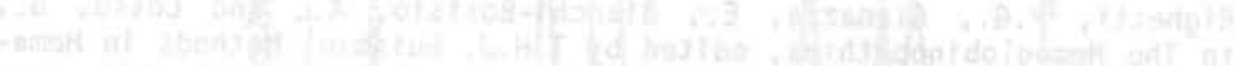

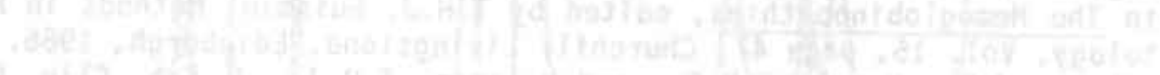

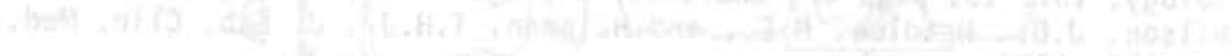

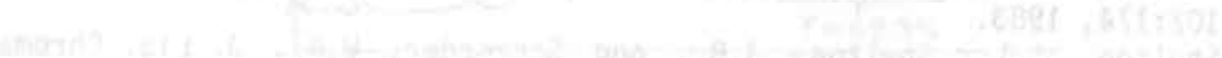

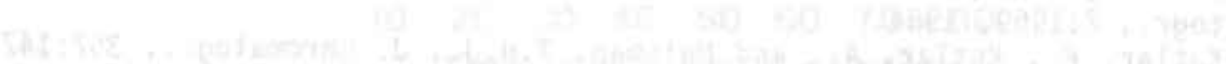

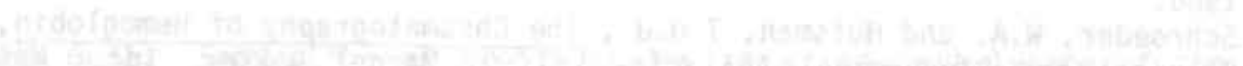

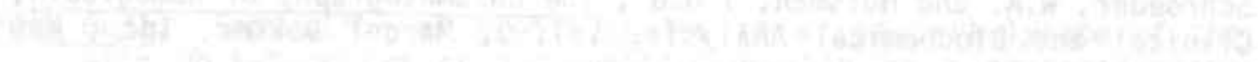

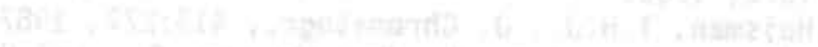

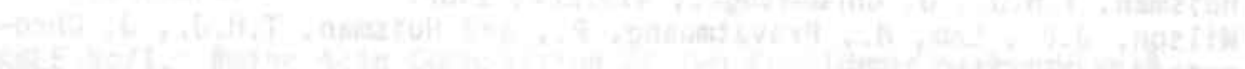

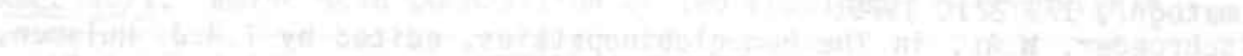

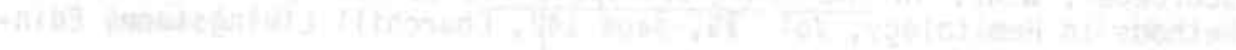

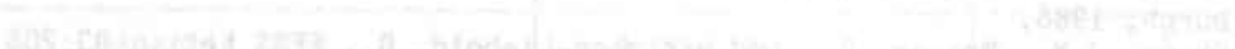

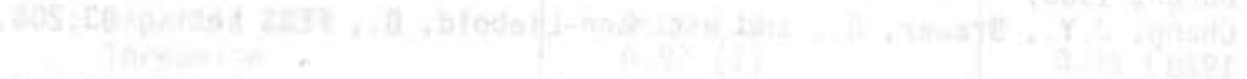

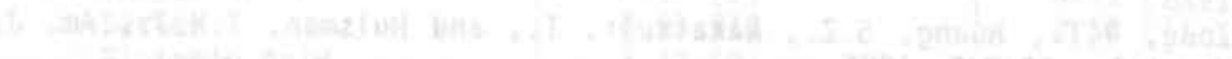

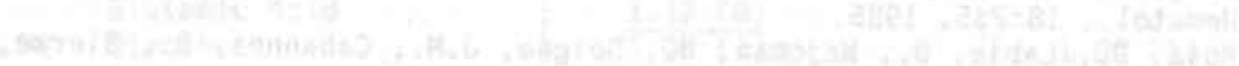

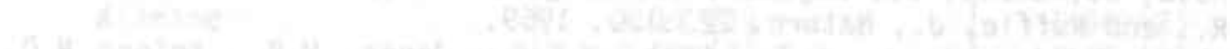

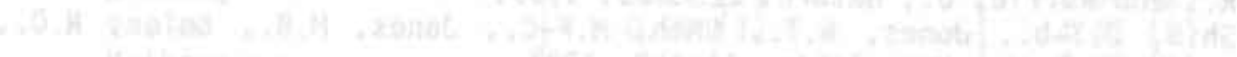

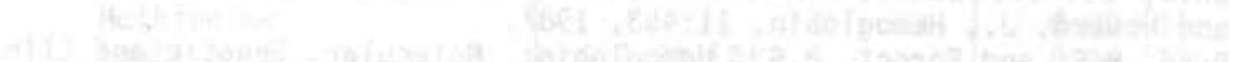

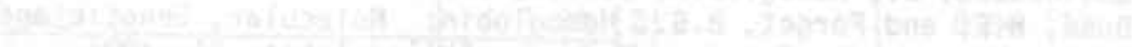

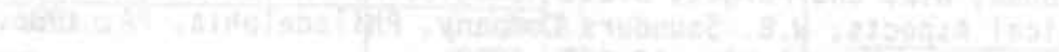

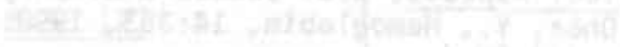


CHAPTER 7

HB MONTREAL, A VARIANT WITH AN EXTENDED в CHAIN DUE TO A DELETION AND AN INSERTION AT THE SAME LOCATION, IDENTIFIED BY SEQUENCING OF AMPLIFIED DNA 


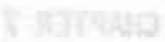

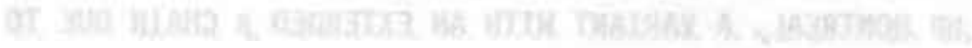

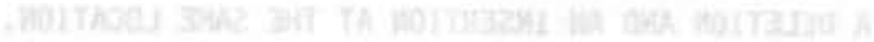

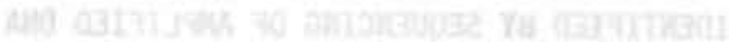




\title{
CHAPTER 7
}

HB MONTREAL, A NEH VARIANT HITH AN EXTENDED B CHAIN DUE TO A DELETION OF ASP,GLY,LEU AT POSITIONS 73, 74, AND 75, AND AN INSERTION OF ALA,ARG,CYS,GLN AT THE SAME LOCATION

\author{
D. Plaseska1, A.J. Dimovski1, J.B. Hilson 1 ,
} B.B. Webber ${ }^{1}$, H.A. Hume ${ }^{2}$, and T.H.J. Hui sman 1

1 Department of Cell and Molecular Biology Medical College of Georgia, Augusta, GA 30912-2100, USA 2 Department of Pediatrics Hôpital Sainte-Justine, Montreal, Quebec H3T 1C5, Canada

(Adapted from: B1ood, 77:178-181, 1991)

\section{ABSTRACT}

The unstable Hb Montreal with a deletion of three amino acid residues (Asp,Gly,Leu) at positions 73,74 , and 75 of the $B$ chain and an insertion of four residues (Ala,Arg,Cys,Gln) at the same location was observed in a 7-year-old Canadian boy suffering from a moderate hemolytic anemia. The introduction of an extra amino acid residue and of other changes in the crevice where the heme group is located is the likely cause of the instability of this $\mathrm{Hb}$ variant. The above listed changes were detected through analyses of tryptic peptides of the B-Montreal chain, sequencing of amplified DNA, and hybridization of amplified DNA with appropriate, 32p-labeled, oligonucleotide probes. It is suggested that a mispairing involving the AGTG sequences at CDs 66 and 67 and at $\operatorname{CDs} 72$ and 73 of the normal $B$ gene caused a repetition of a 16 bp segment, while a deletion of $10 \mathrm{nts}$ due to recombination or slippage, followed by a second short deletion during DNA repair, resulted in the modified sequence of the $\beta$-ilontreal gene. 


\section{INTRODUCTION}

In our continued survey of patients with hemolytic disease caused by the presence of an unstable $\mathrm{Hb}$ variant, we studied a young Canadian patient with a most unusual $\mathrm{Hb}$ abnormality which had a deletion of three amino acids in the $B$ chain and an insertion of four different amino acids at the same location. Here we describe details of this investigation.

\section{MATERIALS AND METHODS}

Blood samples were collected in vacutainers with EDTA as anticoagulant and shipped by overnight mail from Montreal, Canada to Augusta, GA. Informed consent was obtained.

Hematological data were obtained with automated cell counters and with routine hematological procedures. Red cell lysates were studied by IEF (1) with commercially available agar plates (Isolab, Inc., Akron, $\mathrm{OH}$, USA). The abnormal Hb was tested for heat stability (2). The variant was quantitated by cation exchange $\operatorname{HPLC}(3,4)$ and by reversed phase HPLC $(5,6)$.

The variant was isolated by preparative DEAE-cellulose chromatography (2); it eluted behind $\mathrm{Hb} \mathrm{A}_{2}$ in this system. The $\alpha$ and ${ }_{B^{X}}$ chains were separated by a preparative reversed phase HPLC procedure with a large $(21.5 \mathrm{x}$ $300 \mathrm{~mm}) C_{18}$ column (7). The isolated $\mathrm{B} X$ chain was aminoethylated (8) and digested with trypsin for 8 hours at $\mathrm{pH} 8.5$ and at room temperature. The separation of the tryptic peptides was with a reversed phase HPLC procedure (9), while the amino acid composition of each isolated peptide was determined with a Waters Pico Tag amino acid analyzer. Sequence analysis of two peptides was with the method of Chang et al (10).

DNA was isolated by the method of Poncz et al (11). An appropriate segment of genomic DNA was amplified as described before $(12,13)$ and sequenced by the Sanger dideoxy procedure (14). The changes in DNA sequence were verified by dot-blot analysis with $32 \mathrm{p}$-labeled probes with sequences listed in the legend of Fig. 7/3. Methodology has been described in earlier studies $(15,16)$.

\section{RESULTS}

Case Report. The propositus is a 7-year-old male born to a healthy French-Canadian couple. His mother noted scleral icterus at 8 months of age and his anemia was noted at about 15 months. He was first seen at the Hôpital Sainte-Justine in Montreal at $2 \frac{1}{\xi}$ years. Hematological data for him and his parents at the latest collections are listed in Table $7 / 1$. Heinz bodies were readily detectable after 1-2 hours of incubation of blood at $37^{\circ} \mathrm{C}$, suggesting the presence of an unstable $\mathrm{Hb}$ variant. This was confirmed by a heat stability test which showed that some $15-20 \%$ of the $H b$ in a red cell lysate readily precipitated at $61^{\circ} \mathrm{C}$. The Hb variant was also detected by IEF; its mobility was similar to that of a common Hb $D$. 
Quantitation was by two HPLC procedures; the $\beta^{X}$ and $\beta^{A}$ chains were partially separated on a reversed phase $C_{4}$ column (Fig. $7 / 1$, top), while the $H b X$ eluted from a cation exchange HPLC column well separated from the other Hb types (Fig. $7 / 1$, bottom). Its quantity before splenectomy was some $16-19 \%$, and after splenectomy this value had decreased to $12 \%$. The $\mathrm{Hb} \mathrm{A}_{2}$ value of 4 to $4.5 \%$ was definitely higher than normal $(2-3 \%)$; Hb $F$ was also slightly increased (2-3\% versus $<1 \%$ as normal value). The parents of the boy were healthy and had $\mathrm{no} \mathrm{Hb}$ abnormality. There are two other normal children in this family, neither with any anemia.

TABLE 7/1. Hematological Data

\begin{tabular}{l|cc|c|c}
\hline & \multicolumn{2}{|c|}{ Patient F.P. } & Father P. & Mother P. \\
\hline Sex-Age & \multicolumn{2}{|c|}{ M- 7 } & M-31 & F-28 \\
Hb (g/d1) & 8.3 & $8.9 \mathrm{a}$ & 15.5 & 14.5 \\
PCV (1/1) & 0.285 & 0.305 & 0.44 & 0.405 \\
RBC (1012/1) & 2.59 & 2.63 & 5.28 & 4.52 \\
MCV (f1) & 110.0 & 116.0 & 83.0 & 90.0 \\
MCH (pg) & 32.0 & 33.8 & 29.4 & 32.1 \\
MCHC (g/d1) & 29.1 & 29.2 & 35.2 & 35.8 \\
Hb F (\%)b & 3.0 & 2.1 & $<1.0$ & $<1.0$ \\
Hb A2 (\%) & 4.4 & 4.1 & 2.6 & 2.8 \\
Hb X (\%) & 16.7 & 12.0 & 0 & 0 \\
\hline
\end{tabular}

a Data a few weeks after splenectomy.

b By cation exchange $\operatorname{HPLC}(3,4)$.

Structural Analyses. Fig. $7 / 2$ illustrates the separation of the tryptic peptides of the AE- $X$ chain. Nearly all peptides were recovered, had their expected position in the chromatogram, and had normal amino acid compositions. The BT-9 was missing and two small fragments were found; one eluted behind $\beta T-1$ and the second between $\beta T-11$ and $\beta T-13$. Their amino acid compositions are 1 isted in Table $7 / 2$; both fragments were rather pure and probably replaced the first eight and the last eight residues of peptide $B T-9$. Peptide $B T-9 A$ of $H b X$ shared six amino acid residues with $B T-g A$ of $\mathrm{Hb} A$ and an Asp and Gly were replaced by Arg and Ala; sequence analyses identified the five amino terminal residues as being the same as those found for $B T-9$ ( $H b A)$. Peptide $B T-9 B(H b X)$ shared seven residues with the $C$-terminal segment of $B T-9(H b A)$, contained one Cys residue and one Glu (or Gln) residue not present in $B T-9 B(H b A)$, and had one less Leu residue. Its sequence determination was complicated by a probable -cysGin- amino terminus and by a low recovery of the peptide. From these results we derived the tentative amino acid sequence for the $B$ T- 9 A and $B$ fragments as listed in Fig. $7 / 2$. The suggested sequence of $B T-9(H b X)$ compared with that of $\mathrm{Hb} A$ was as follows:

\section{$\begin{array}{llllllllllllllll}67 & 68 & 69 & 70 & 71 & 72 & 73 & 74 & 75 & 76 & 77 & 78 & 79 & 80 & 81 & 82\end{array}$}

Hb A: Val-Leu-Gly-Ala-Phe-Ser---Asp-Gly-Leu---Ala-His-Leu-Asp-Asn-Leu-Lys Hb $X$ : Val-Leu-Gly-Ala-Phe-Ser-ALA-ARG-CYS-GLN-Ala-His-Leu-Asp-Asn-Leu-Lys

These preliminary data suggested a deletion of the three amino acid residues (Asp,Gly,Leu) and an insertion of four amino acid residues (Ala, Arg, (ys, GIn). 

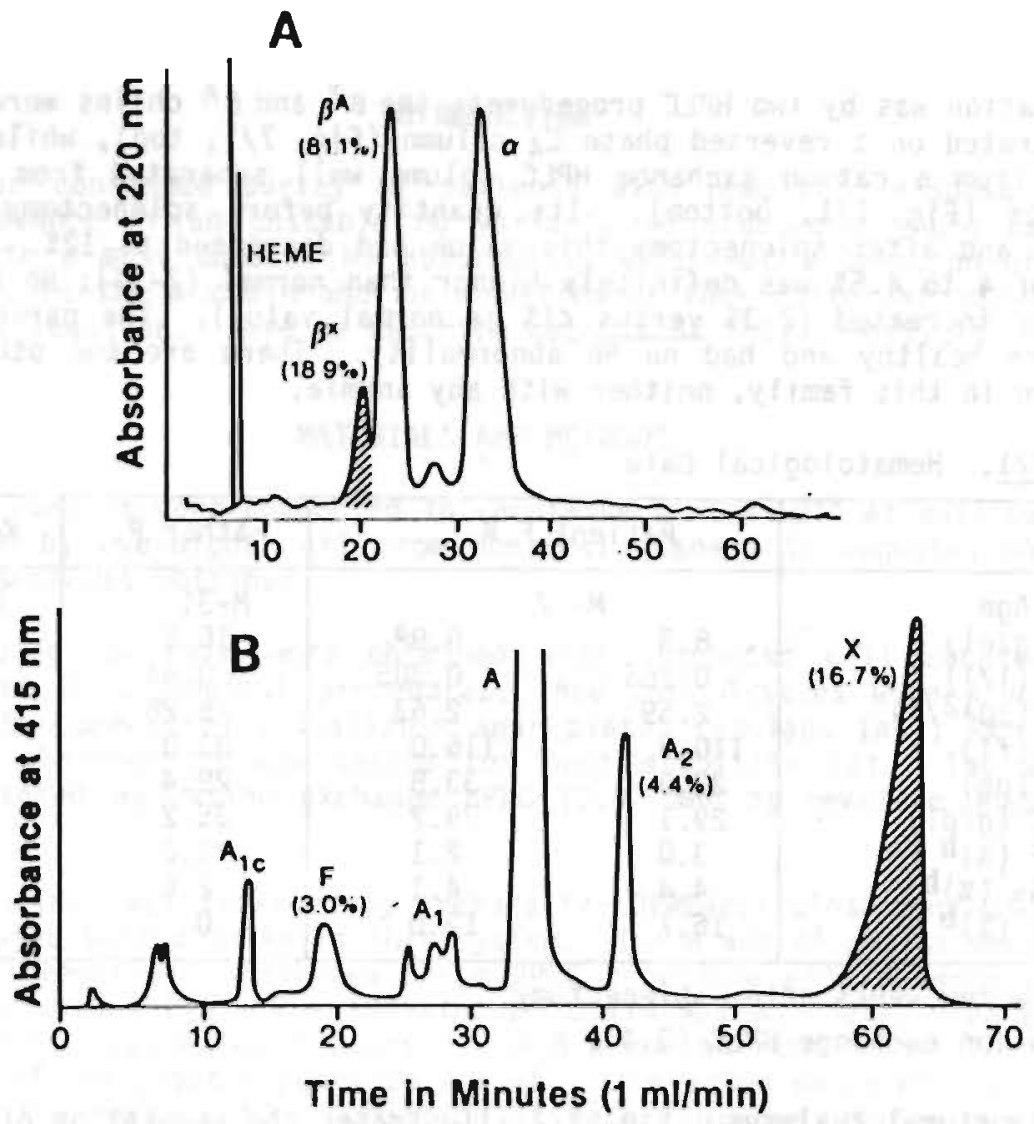

FIG. 7/1. Identification of Hb Montreal by HPLC. Top: Partial separation of the $\beta X(=\beta$-Montreal) and $\beta A$ chains in reversed phase $\operatorname{HPLC}(5,6)$. Bottom: Separation of $\mathrm{Hb} \times(=\mathrm{Hb}$ Montreal) and the other $\mathrm{Hb}$ components by cation exchange $\operatorname{HPLC}(3,4)$.

TABLE 7/2. Amino Acid Composition of the Two Fragments of Peptide T-ga

\begin{tabular}{llc}
\hline Amino Acid & \multicolumn{1}{c}{$T-9^{A}$} & $T-9^{B}$ \\
\hline Aspartic Acid & $0 \quad(1)$ & $1.97(2)$ \\
Glutamic Acid & $0.84(1)$ & $1.13(0)$ \\
Serine & $0.93(3)$ & \\
Glycine & $\underline{1.14(0)}$ & \\
Arginine & $2.17(1)$ & $0.78(1)$ \\
Histidine & $0.95(1)$ & $0.88(1)$ \\
Alanine & $0.97(1)$ & $1.88(3)$ \\
Valine & $0.97(1)$ & $1.00(1)$ \\
Leucine & & $0.68(0)$ \\
Phenylalanine & & $75-82$ \\
Lysine & $67-74$ & \\
AE-Cysteine & & \\
\hline Positions in normal B chain & &
\end{tabular}

a In residues/mole. The numbers between parentheses refer to the numbers of residues observed for the positions in the normal $B A$ chain. 


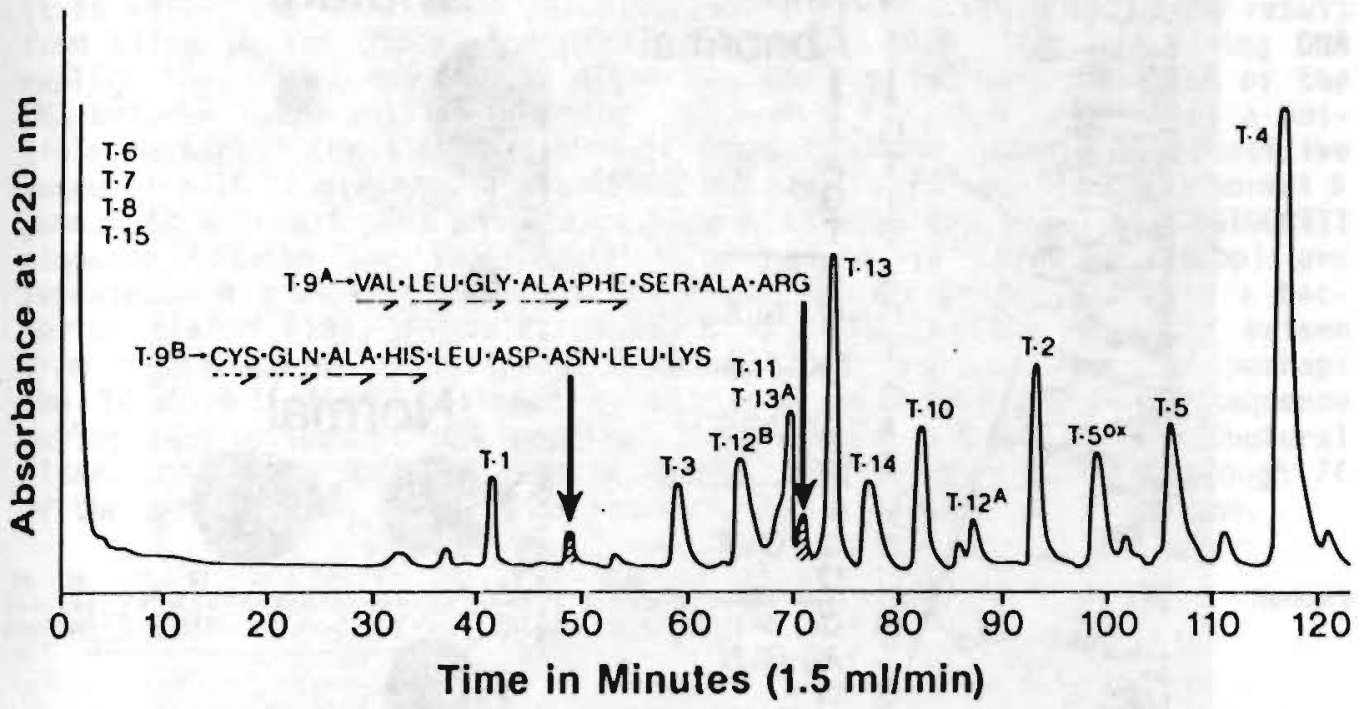

FIG. $7 / 2$. Separation of peptides in a tryptic digest of the $A E-\beta X \quad(=\beta-$ Montreal) chain by reversed phase HPLC (9). The abnormal peptides indicated by peaks with dashed lines; the insert shows the sequence of these peptides.

DNA Analyses. The DNA from the patient was amplified asymmetrically and sequenced in both directions. The data identified a frameshift starting at $C D 73$ with the direct sequencing primer, and at $C D 75$ with the reverse sequencing primer (Fig. 7/3). This made it possible to characterize the sequences of the normal chromosome and the abnormal chromosome as indicted in Fig. $7 / 3$. These data confirmed the tentative results obtained by the analyses of the abnormal ${ }_{B} X$ chain. Final confirmation was obtained by dot-blot analyses of amplified DNA from the patient and his parents with 32p-labeled oligonucleotide probes; the deletion/insertion was identified only in the DNA of the patient (Fig. 7/3). The Hb variant was named $\mathrm{Hb}$ Montreal after the city of residence of the propositus.

\section{DISCUSSION}

Hb Montreal is clearly a most unusual $\mathrm{Hb}$ variant because its abnormal $B$ chain has a deletion of three amino acid residues (Asp,Gly, Leu at B 73, $B 74$, and B75) and an insertion of four different residues (Ala,Arg,Cys,Gln) at the same location. The evidence is based on data obtained through chemical analysis of two isolated tryptic fragments of the $B$ chain ( $T-g A$ and $\left.T-g^{B}\right)$, on sequence data of amplified DNA, and finally, on data from hybridization of the amplified DNA with 32p-labeled probes specific for this change in DNA sequence. The location of this deletion/insertion is of some interest because two other $B$ chain variants have been discovered which are characterized by a deletion of Leu at $B 75$ [Hb Vicksburg (17)] and of Gly-Leu at B 74 and $B 75$ [Hb St. Antoine (18)]. 


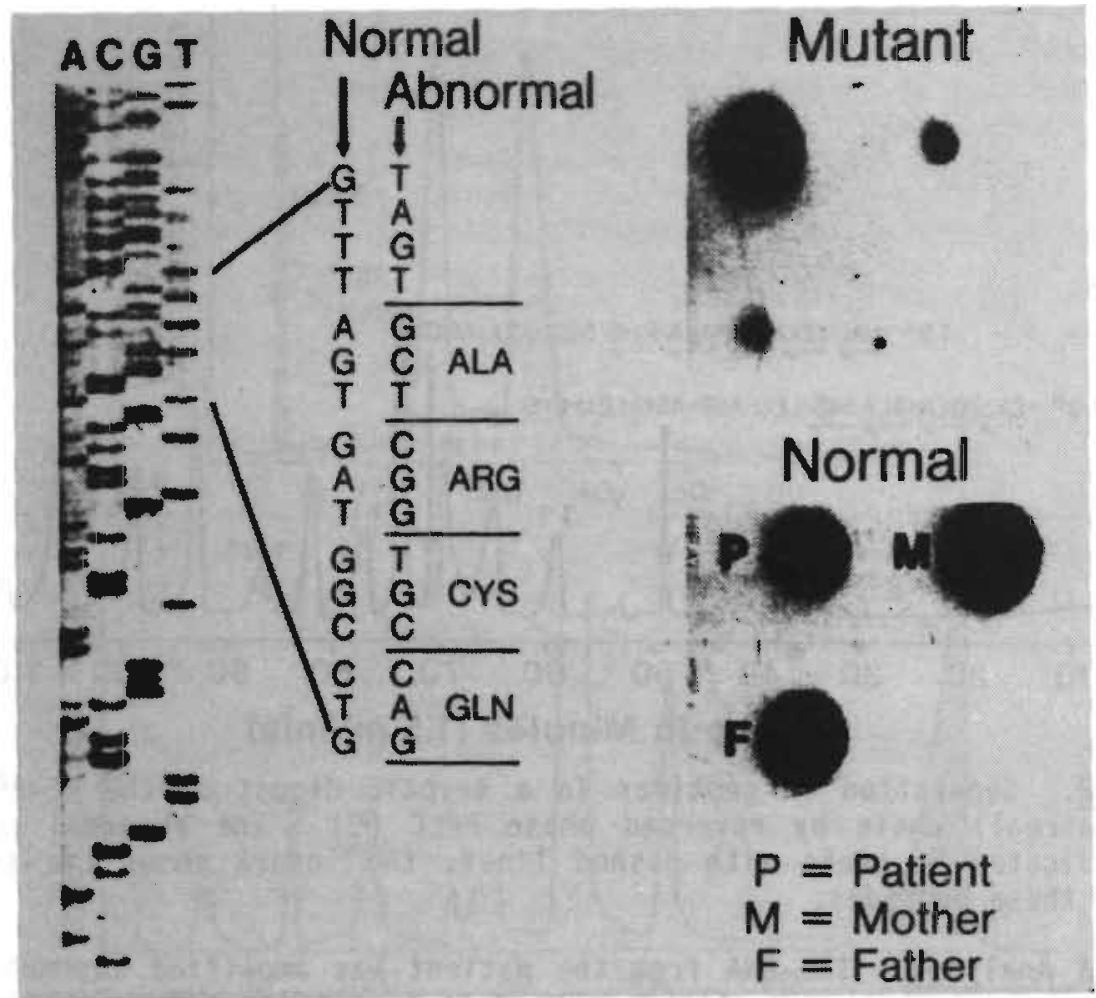

FIG. 7/3. Left: Reverse sequence analysis of amplified DNA of the propositus F.P. The start of the double-banding is located in CD 76 (CAG for Gln) in ${ }_{\beta} X$ and CD 75 (CTG for Leu) in $\beta A$. The direct sequence identified two CDs at position 73 (GCT for Ala in $\beta$ $X$ and GAT for Asp in $\beta A$; data not shown). The sequences of the amplification primers were: Direct: 5'-GCCAAGGACAGGTACGGCTGTCATC-3' (positions -140 through -116 relative to the Cap site); reverse: 5'-CCCTICCTATG ACATGAACTTAACCAT- $3^{\prime}$ (positions +566 through +540 relative to the Cap site), Right: The results of the hybridization of amplified DNA with 32 - Tabeled probes; the sequences of the probes were: Mutant: 5'-AGTGCTCGGTGCCAGGCTC-3' ; norma 1: 5'-AGTGATGGCCTGGCTCACC-3' .

The deletion of Asp-Gly-Leu and the insertion of Ala-Arg-Cys-Gln occurred at the C-terminal end of the E helix (positions E17, E18, E19) which is part of the crevice where the heme group is located. Although none of the three deleted residues is involved in any major contact, i.e. With heme or between chains, it is evident that the introduction of an extra amino acid in this helix, and the presence of the large arginine residue should affect the stability of the protein and perhaps its function. A considerable instability of the variant was indeed noticed by hematological observations such as Heinz body formation and an increase in precipitation of $\mathrm{Hb}$ during the heat stability test. MetHb (= ferriHb) was not observed and functional studies were not conducted.

The $B$-Montreal variant is presumed to be the result of a de novo genetic event because the parents of this young patient were normal and did not have the Hb variant; paternity testing was not performed. The mechanism(s) responsible for the noted changes is (are) not clear. However, 
it is well-known that small deletions and also small insertions can result from slippage and mispairing of two homologous DNA sequences during DNA replication. This may result either in the removal or repetition of the DNA between these small homologous sequences. Fig. $7 / 4$ illustrates a possible mechanism for the formation of the $B$-Montreal gene. The repetitive sequence AGTG is present in CDs 66 and 67 and CDs 72 and 73 of the normal $B$ gene. As a result of a mispairing, the AGTG sequence and the CTCGGTGCCTTI sequence between the two repetitive sequences (a total of $16 \mathrm{bp}$ ) are repeated. A similar phenomenon has been extensively described in a bacterial system (19). A deletion of $10 \mathrm{nts}$ (TGATGGCCTG) may have arisen from recombination or slippage between short sequence repeats (perhaps the TG dinucleotide) followed by a second deletion of the TIT sequence during the subsequent DNA repair. The final result of these structural alterations is a modified sequence which affects only CDs 73 through 76 of the B-Montreal gene and does not otherwise alter the reading frame.

$\begin{array}{llllllllllllllll}65 & 66 & 67 & 68 & 69 & 70 & 71 & 72 & 73 & 74 & 75 & 76 & 77 & 76 & 79 & 60\end{array}$

LYs-Lys-Vai-Lou-GIY-AIa-Pho-Ser-Asp-GIy-LOU-AIa-His-Lou-AsD-Asn

$M G-A A-G T G-C T C-G G T-G C C-T T-A G T-G A T-G G C-C T G-G C T-C A C-C T G-G A C-A A C$

Normal p

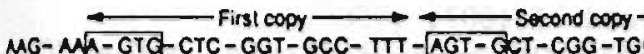
lys-Lys-Val-Leu-GIy-Ala-Pho-Ser-ALA-ARG-CYS

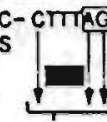

"Intermedlate consinct"

GCT-CAC-CTG-GAC-AAC Ala-His-Lou-AsD-Asn Doletions

$\begin{array}{lllllllllllllllll}65 & 66 & 67 & 60 & 69 & 70 & 71 & 72 & 73 & 74 & 75 & 76 & 77 & 78 & 79 & 80 & 81\end{array}$

$M G-M A-G T G-C I C-G G T-G C C-T T$ - AGT-GCT-CGG-TGC-CAG-GCT-CAC-CTG-GAC-AM

Lys-Lys-Val-Lou-GIy-AI a-Pho-Ser-ALA-ARG-CYS-GLN-Ala - His - Lou - Asp-Asn

Monireal $\beta$

FIG. 7/4. A scheme providing a possible mechanism for the formation of the B-Montreal gene.

Acknowledgements. This study was supported in part by USPHS research grants HLB-05168 and HBL-41544. This is contribution \#1260 from the Department of Cell and Molecular Biology at the Medical College of Georgia in Augusta, GA.

\section{REFERENCES}

1. Righetti PG, Gianazza E, Bianchi-Bosisio A, Cossu G: Conventional isoelectric focusing and immobilized $\mathrm{pH}$ gradients for hemoglobin separation and identification, in Huisman THJ (ed): The hemoglobinopathies, Vo1. 15. Edinburgh, Churchill Livingstone, 1986, p 47

2. Huisman THJ, Jonxis JHP: The Hemoglobinopathies Techniques of Identification. New York, Marcel Dekker, 1977

3. Bisse E, Wieland H: High-performance liquid chromatographic separation of human haemoglobins - Simultaneous quantitation of foetal and glycated haemoglobins. J Chromatogr 434:95, 1988

4. Kutlar A, Kutlar F, Gu L-G, Mayson SM, Huisman THJ: Fetal hemoglobin in normal adults and $B$-thalassemia heterozygotes. Hum Genet 85:106, 1990

5. Shelton JB, Shelton JR, Schroeder WA: High performance liquid chromatographic separation of globin chains on a large-pore $C_{4}$ column. J Liq Chromatogr 7:1969, 1984 
6. Kutlar F, Kutlar A, Huisman THJ: Separation of normal and abnormal hemoglobin chains by reversed-phase high-performance liquid chromatography. J Chromatogr 357:147, 1986

7. Huisman THJ: Separation of hemoglobins and hemoglobin chains by high-performance liquid chromatography. J Chromatogr 418:277, 1987

8. Jones RT: Structural studies of aminoethylated hemoglobins by automatic peptide chromatography. Cold Spring Harbor Symposium. Quant Biol 29:297, 1964

9. Wilson JB, Lam H, Pravatmuang P, Huisman THJ: Separation of tryptic peptides of normal and abnormal $\alpha, \beta, \gamma$, and $\delta$ hemoglobin chains by high-performance liquid chromatography. J Chromatogr 179:271, 1979

10. Chang JY, Brauer D, Wittmann-Liebold, B: Micro-sequence analysis of peptides and proteins using 4-NN-dimethylaminoazobenzene $4^{\prime}$-isothiocyanate/phenylisothiocyanate double coupling method. FEBS Lett 93:205, 1978

11. Poncz M, Solowiejczyk D, Harpel B, Mory Y, Schwartz E, Surrey S: Construction of human gene libraries from small amounts of peripheral blood: Analysis of $B-1$ ike globin genes. Hemoglobin 6:27, 1982

12. Gonzalez-Redondo JM, Stoming TA, Lanclos KD, Gu YC, Kutlar A, Kutlar F, Nakatsuji T, Deng B, Han IS, MCKie VC, Huisman THJ: Clinical and genetic heterogeneity in Black patients with homozygous $B$-thalassemia from the Southeastern United States. Blood 71:1007, 1988

13. Gonzalez-Redondo JM, Stoming TA, Kutlar F, Kutlar A, McKie VC, McKie KM, Huisman THJ: Severe Hb $S / B^{\circ}$-thalassaemia with a $T->C$ substitution in the donor splice site of the first intron of the $B-g l o b i n$ gene. Br J Haematol 71:113, 1989

14. Sanger $F$, Nicklen S, Coulson AR: DNA sequencing with chain terminating inhibitors. Proc Natl Acad Sci USA 74:5463, 1977

15. Saiki RK, Gelfand DH, Stoffel S, Scharf SJ, Higuchi R, Horn GT, Mullis KB, Erlich HA: Primer-directed enzymatic amplification of DNA with a thermostable DNA polymerase. Science 239:487, 1988

16. Diaz-Chico JC, Yang KG, Yang KY, Efremov DG, Stoming TA, Huisman THJ: The detection of $\beta-g l o b i n$ gene mutations in $\beta$-thalassemia using oligonucleotide probes and amplified DNA. Biochim Biophys Acta 949:43, 1988

17. Adams JG III, Steinberg MH, Newman MV, Morrison WT, Benz EJ Jr, Iyer $R$ : $B$-Thalassemia present in cis to a new $\beta$-chain structural variant, Hb Vicksburg [B75 (E19) Leu->0]. Proc Nat1 Acad Sci USA 78:469, 1981

18. Wajcman $H$, Labie D, Schapira G: Two new hemoglobin variants with deletion. Hemoglobin Tours: Thr B 87 (F3) deleted and Hemoglobin St. Antoine: Gly-Leu B 74-75 (E18-19) deleted. Consequences for oxygen affinity and protein stability. Biochim Biophys Acta 295:495, 1973.

19. Farabaugh PJ, Schmeissner U, Hofer M, Miller JH: Genetic studies of the lac repressor. VII. On the molecular nature of spontaneous hotspots in the LacI gene of Escherichia coli. J Mol Biol 126:847, 1978 
CHAPTER 8

DISCUSS I ON 
C:

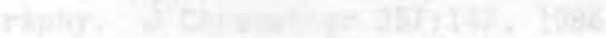

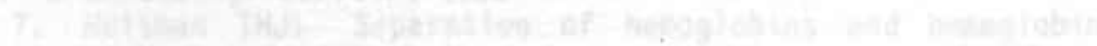

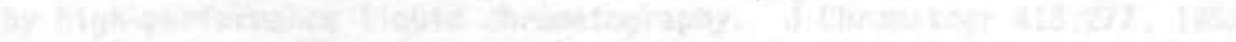

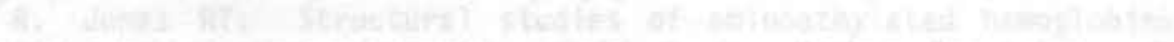

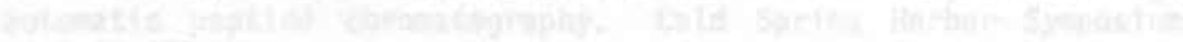

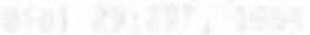

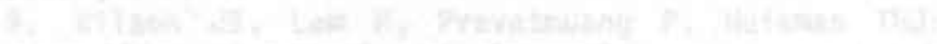

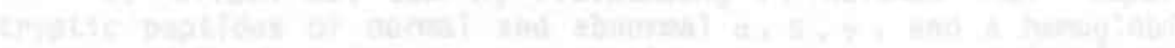

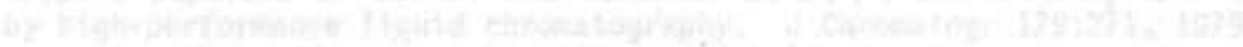

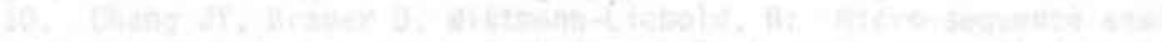

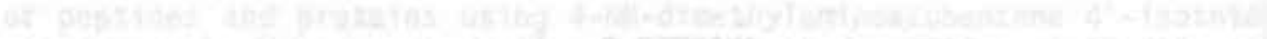

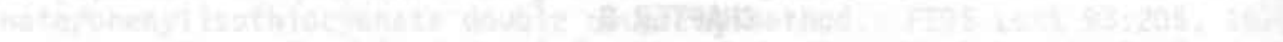

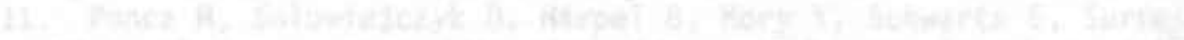

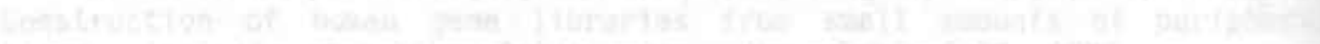

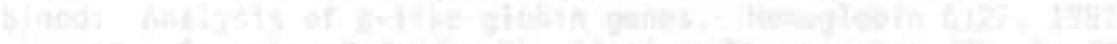

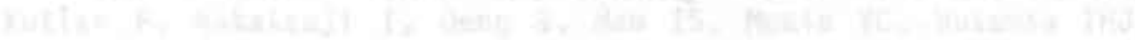

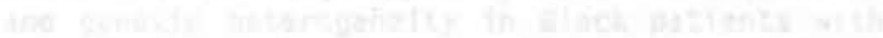

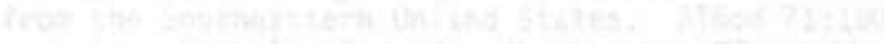

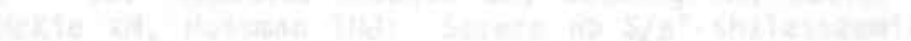

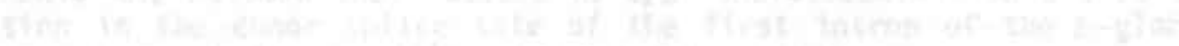
(17.

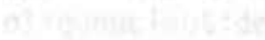

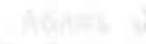

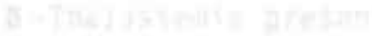

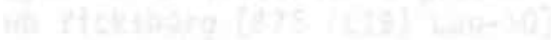

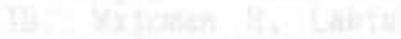

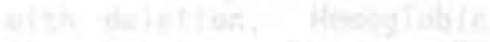

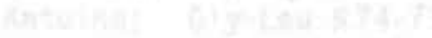

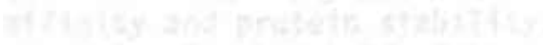

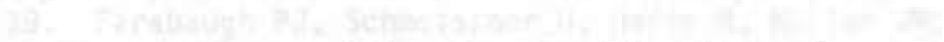

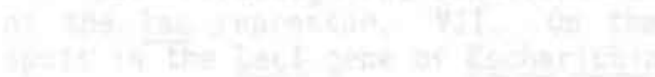




\section{CHAPTER 8}

\section{DISCUSSION}

Since the discovery of $\mathrm{Hb} S$ in 1949 (1), more than $600 \mathrm{Hb}$ variants have been described (2). The extensive progress made in the studies of Hb variants was made possible primarily by technological advances, namely the development of more sensitive and rapid methods for their detection and characterization. Most of the common $\mathrm{Hb}$ variants were detected some 20 to 30 years ago because of their altered electrophoretic mobilities. Hb variants that have been described in recent years are usually rare abnormalities, that have mutations that are difficult to detect by electrophoretic procedures or a rather complex structure requiring advanced methodology for their characterization. Fig. $8 / 1$ shows the number of different Hb abnormalities detected between 1978 and 1992. The increase in the number of $H \mathrm{~b}$ variants with amino acid deletions or extended chains, of $\gamma$ and $\delta$ chain variants, and of those with two mutations that were detected in the last few years is likely due to the application of improved methodology.

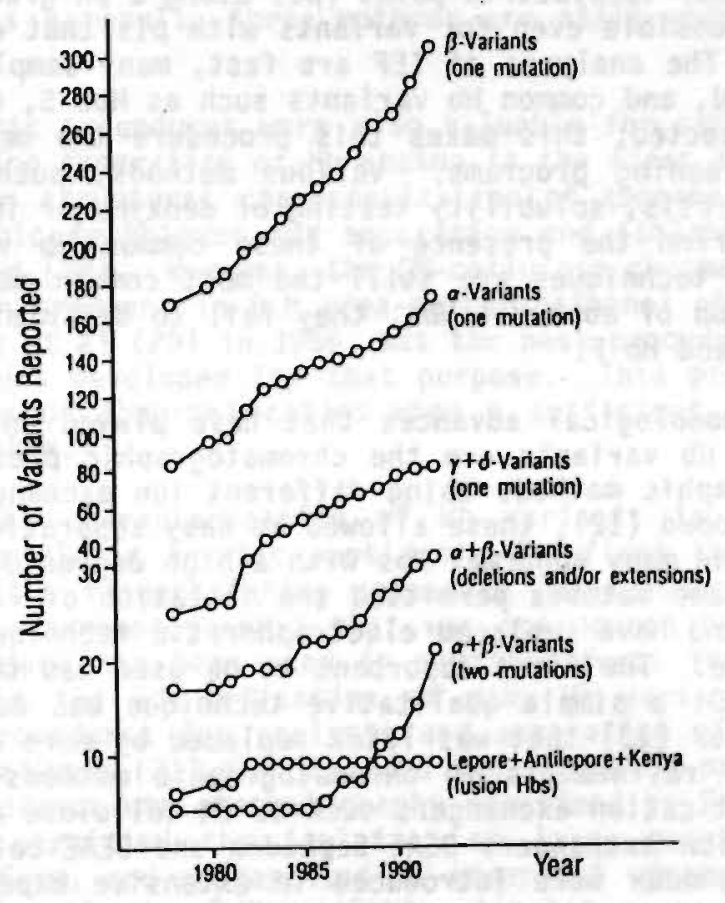

FIG. 8/1. The number of different Hb variants detected between 1978 and 1992 (from Ref. 3).

Technological Advances in the Studies of Hb Variants. Electrophoretic techniques have unquestionably played an important role in the studies of $\mathrm{Hb}$ variants. The first electrophoretic technique was the moving boundary type that was conducted in a liquid media and was used in the original 
study of Pauling and his colleagues when they discovered the abnormal electrophoretic behavior of $\mathrm{Hb} S$ (1). This discovery stimulated a search for other Hb abnormalities, and within a short time, two new Hb variants with altered electrophoretic mobilities, namely $\mathrm{Hb} \mathrm{C} \mathrm{(4)} \mathrm{and} \mathrm{Hb} D$ (5) were described, and many others thereafter. Although the moving boundary electrophoresis offered a high degree of accuracy and precision, it was timeconsuming and difficult to perform. This, and an increased interest in the abnormalities, stimulated a search for more simple electrophoretic techniques that could be used for screening purposes. The development of the electrophoretic technique that uses filter paper as a support medium $(6,7)$ permitted a larger number of $\mathrm{Hb}$ variants to be detected. Improvements in electrophoretic techniques have included the use of starch gel (8), agar gel (9), and other support media, as well as the development of IEF methods (10). Different modifications of these techniques have been introduced that have permitted higher resolution of $\mathrm{Hbs}$ and have contributed significantly to the identification of many abnormal Hbs.

Currently, a large number of electrophoretic techniques are available; most popular is IEF, that has a high resolving power and the ability to detect minor $\mathrm{Hb}$ components. In IEF, the different $H b s$ are fractionated according to their isoelectric point ( $P I)$ along a pH gradient; an excellent resolution is possible even for variants with $p$ Is that differ by only 0.02 $\mathrm{pH}$ unit (11). The analyses of IEF are fast, many samples can be simultaneously analyzed, and common $\mathrm{Hb}$ variants such as Hbs S, C or G-Philadelphia are readily detected; this makes this procedure the method of choice for large-scale screening programs. Various methods, such as acid citrateagar electrophoresis, solubility testing of deoxyHb or immunological procedures, can confirm the presence of these common Hb variants. Although electrophoretic techniques are still the most common methods used for the initial detection of abnormal Hbs, they fail to discriminate many Hb variants from $\mathrm{Hb} A$ and $\mathrm{Hb} F$.

Other methodological advances that have played an important role in the studies of $\mathrm{Hb}$ variants are the chromatographic procedures. Different macrochromatographic methods using different ion exchangers and developers have been developed (12); these allowed an easy separation and quantitation of the normal and many abnormal Hbs with a high degree of resolution. Furthermore, the same methods permitted the isolation of various abnomal Hbs in pure form, and have replaced electrophoretic techniques previously used for that purpose. The first absorbent to be used was the cation exchanger Amberlite IRC-50; a simple qualitative technique was developed by Huisman and Prins in 1957 (13) that was later replaced by more accurate procedures $(14,15)$. Later refinements in chromatographic methods have included the use of different cation exchangers such as CM-cellulose and CM-Sephadex, as well as the anion exchangers DEAE-Sephadex and DEAE-cellulose. CM-cellulose and CM-Sephadex were introduced in extensive experiments by Huisman and his collaborators (16-19). DEAE-Sephadex was used for the first time by Dozy et al in 1968 (20) and improved by Huisman and Dozy in 1969 (21); almost immediately this procedure became the most popular chromatographic method for quantitation and isolation of abnormal Hbs. The later developed DEAE-cellulose chromatographic method with glycine-KCN-NaCl developers (22) offered additional advantages; it was faster, simpler, and provided 
improved separation of $\mathrm{Hbs}$. This method has replaced all other procedures for the quantitation and isolation of $\mathrm{Hb}$ variants, and in many laboratories, is still widely used for the isolation of larger quantities of certain abnormal Hbs. Although all these chromatographic methods are reproducible and offer quantitative data, they have the disadvantage of being time-consuming and labor-intensive, thus making them unsuitable for screening purposes.

The search for a rapid and improved methodology for the quantitation of $\mathrm{Hb} \mathrm{A} \mathrm{A}_{2}$, and the detection and quantitation of some common abnormal $\mathrm{Hbs}$ such as HbS $S$ and $C$, led to the development of several microchromatographic procedures. Chromatography on small columns was first described by Huisman and Dozy in 1962 (23), but the micro procedures became really popular in 1973 when Schroeder, Huisman, and their collaborators introduced miniature columns and developing systems useful for a rapid detection of $\mathrm{Hbs} S$ and $C$ at birth (24), and for the quantitative determination of $\mathrm{Hb} \mathrm{A}_{2}(25,26)$. Since then, other microchromatographic methods or modifications of existing ones have been devised for the quantitation of different $H b$ types such as Hbs $A_{2}, F, B a r t ' s$, and $A_{1 c}$ (27). Although these microchromatographic techniques are less accurate for the detection and quantitation of the major abnormal Hbs, they are most useful for a rapid and accurate quantitation of $\mathrm{Hb} \mathrm{A} A_{2}$. As a result, these methods are still widely used in many laboratories.

Chromatographic procedures were also valuable for structural analyses of $\mathrm{Hb}$ variants. The separation of $\mathrm{Hb}$ chains is the first important step in the procedures for structural characterization of abnormal globin chains; many chromatographic techniques for separation and isolation of $\mathrm{Hb}$ chains have been described (28). However, the CM-cellulose chromatographic method using a sodium-ion gradient in $8 \mathrm{M}$ urea-mercaptoethanol phosphate buffers, developed by Clegg et al (29) in 1966, was the best procedure that replaced all other techniques developed for that purpose. This procedure is still used for isolation of abnormal chains when a sufficient amount of sample material is available.

The structural characterization of $\mathrm{Hb}$ variants is usually done by examination of the globin tryptic peptides. The first technique used for this purpose was the fingerprinting procedure (paper electrophoresis followed by partition chromatography) that was introduced by Ingram in 1956 (30). This procedure has been used in modified forms for many years and has contributed to the identification of many Hb variants. Macrocolumn chromatographic procedures for analysis and separation of globin peptides were introduced by Jones (31) and included the automatic peptide chromatography and Dowex macrocolumn chromatography described by Schroeder $(32,33)$. These techniques permitted the isolation of large quantities of globin peptides in pure form, and represented an important advance in methodology for the structural analyses of $\mathrm{Hb}$ variants.

The development of HPLC methods has brought great improvement in the studies of $\mathrm{Hb}$ abnormalities because highly sensitive and rapid procedures became available for the detection, quantitation, and isolation of abnormal Hbs, globin chains, and peptides. These techniques are accurate and repro- 
ducible, rapid, sensitive, and have high resolving power. Therefore, they have rapidly replaced all other available technology for the detection, quantitation, and structural characterization of $\mathrm{Hb}$ variants.

An anion exchange HPLC procedure for the detection and quantitation of normal and abnormal Hbs was first described by Gooding et al in 1979 (34). Several modifications appeared in the literature during the next few years $(35,36)$. This procedure provided a rapid method for the quantitation of Hbs $A, S, C$, and $F$ in newborn and adult samples, as well as for the separation and quantitation of many other abnormal Hbs. The disadvantage of this technique is its inability to separate $H b F$ from the minor $\mathrm{Hb} A_{1}$ components, thus preventing the quantitation of $\mathrm{Hb} F$ in adult samples with a low Hb $F$ percentage.

Cation exchange HPLC appeared to be a more promising method for the separation and particularly for the quantitation of normal and abnormal Hbs $(37-40)$. The general rule defined by macro cation exchange chromatography applies, i.e. the higher the positive charge, the longer the elution time. However, the analyses of numerous $\mathrm{Hb}$ variants with the same type or with comparable amino acid substitutions have shown some differences in their RET values, suggesting that the location of the substitution can affect the elution of $\mathrm{Hb}$ variants in this type of chromatography. Variants due to substitutions within the helical region elute faster than those in a prehelical region or in an interhelical segment. Additional factors, such as an extra positive charge on some of the globin chains, and the formation of salt bonds that eliminate the extra positive charge, may also influence the elution of some Hb variants in this system (41). The differences in RET values are reproducible, allowing the chromatographic identification of certain common $\mathrm{Hb}$ abnormalities; their presence can be confirmed by some of the methods mentioned above.

Preparative anion and cation exchange HPLC columns are also available; the value of these columns is that they allow separation and isolation of $\mathrm{Hb}$ components that are present in very small amounts. For instance, the preparative polyCAT HPLC column allows the complete separation of $\mathrm{Hb} F$ and $\mathrm{Hb} \mathrm{A}_{1 \mathrm{C}}$, and the isolation of $\mathrm{Hb} F$ in pure form when $\mathrm{Hb}$ levels in the samples are as low as 1 or $2 \%(40)$. Thus, this procedure is important for the isolation of some abnormal Hbs that are present in small amounts; several chromatograms can provide sufficient material for the structural characterization by sensitive HPLC procedures.

Different reversed phase HPLC procedures for the separation of $H \mathrm{H}$ chains have been developed $(42-44)$ and the one introduced by Shelton et a) (45), using a large-pore $C_{4}$ column and acetonitrile-water-TFA developers has replaced all other methods. Separation by this procedure is based on the differences in the polarities, sizes, and pK values between the substituted and introduced amino acids. As a general rule, the more polar (less hydrophobic) chains elute earlier in the chromatogram. There is an excellent correlation between predicted (according to the polarities of the amino acids) and obtained RET values, although there are some exceptions, suggesting that some additional factors such as the location of the amino acid substitution in the globin chain may influence the elution 
of the variant globin chain (41). The usefulness of this method in detecting $\mathrm{Hb}$ variants with neutral substitutions has been confirmed many times in recent years, and has resulted in the detection of an increased number of $\mathrm{Hb}$ variants with neutral substitutions.

Preparative reversed phase HPLC columns for chain separation are also available; they have facilitated the isolation of some abnormal globin chains that have neutral substitutions and/or are present in small amounts. This procedure is particularly valuable for the isolation of the $\gamma$ chains in pure form.

A reversed phase HPLC method for the separation of globin peptides was introduced by Wilson et al in 1979 (46). The resolution of peptides in this method is, in part, based on differences in molecular weight, on the hydrophobic nature of the compounds, and to a much lesser extent, to differences in charge. This procedure has shown distinct advantages over the other procedures for peptide separation; it allows separation of micro quantities of tryptic peptides within a short time. Most peptides eluted are pure and uncontaminated, and can be analyzed without further purification with some of the sensitive amino acid analyzers presently available. Small changes in the elution profile can be observed and are frequently indicative of a specific peptide being abnormal in structure. This procedure, and modifications introduced later (47), have greatly facilitated the identification of numerous $\mathrm{Hb}$ variants.

Application of DNA Methodology to the Identification of Hb Variants. Since the genetics of the normal human $\mathrm{Hbs}$ and their variants were well defined long before the development of the recombinant DNA technology, $\mathrm{Hb}$ has served as a model for the implementation of recombinant DNA technology to human genetics. Globin genes were one of the first human genes that were cloned and isolated from DNA libraries, resulting in an early characterization of the chromosomal arrangement of the globin genes and their fine structure. In the same time, the application of recombinant DNA technology has dramatically increased the possibilities for the diagnosis and prenatal detection of the hemoglobinopathies.

Restriction enzyme mapping, or gene mapping, was developed by Southern in 1975 (48), and was one of the first techniques used in the studies of globin genes. The well-defined restriction enzyme map of the $\beta-g l o b i n$ gene cluster allowed the application of this technique to the identification of hybrid Hbs. Gene mapping analyses readily detected the deletions of 7 and $22.5 \mathrm{~kb}$ DNA fragments in the Lepore $\mathrm{Hbs}$ and $\mathrm{Hb}$ Kenya, respectively $(49,50)$. These analyses have brought additional evidence that the hybrid chains of the Lepore and anti-Lepore Hbs are the products of non-equal homologous crossovers between misaligned chromosomes, and have also allowed the more precise localization of the recombination event (51).

Gene mapping analyses can also be used for the identification of abnormal Hbs due to single nt changes that result in the loss or creation of a restriction enzyme site. The first abnormal $\mathrm{Hb}$ that was detected by gene mapping analyses was $\mathrm{Hb} S$. The A->T substitution in the sixth $C D$ of the ${ }_{\beta} S$ gene eliminates the recognition site for the restriction enzymes 
Dde I and Mst II. This approach has been employed for the prenatal diagnosis of $\mathrm{Hb} \mathrm{S}(52)$, and for the identification of several other $\beta$, and even some $Y$, chain abnormal Hbs $(53,54)$. Although the technique represented a great improvement over existing methods, particularly for prenatal diagnosis, it was still rather time-consuming.

The discovery of the amplification PCR procedure $(55,56)$ has had a major impact on the analyses and diagnoses of the hemoglobinopathies. The introduction of this technique has led to a variety of new approaches for rapid and accurate identification of the globin gene mutations. The technique allows the amplification of a target DNA segment from small amounts of DNA over a period of a few hours. The amplified DNA fragment can be directly analyzed in an ethidium bromide stained gel, with allele-specific oligonucleotide probes, by restriction enzyme digestion, or by direct sequencing. These techniques, all widely used for detecting $\alpha$ - and $\beta$-thal alleles, have also been applied to the studies of Hb variants.

PCR has provided a new approach for the detection of the Lepore Hbs. Using a combination of $\delta$ - and $\beta$-specific amplification primers, it is possible to directly detect the Lepore $H b s$ by electrophoretic analys is of the PCR products, provided amplification primers are located at either side of the deletion.

Hb variants that alter restriction enzyme sites can be easily and rapidly detected by digestion of the amplified DNA with these enzymes. The PCR method is so powerful, and it produces so much DNA, that the fragments obtained after digestion with restriction enzymes can be analyzed directly on the ethidium bromide stained gel.

Another approach that facilitates the detection of known mutations is a combination of PCR and hybridization with ASO probes (57). Hybridization with ASO probes was first applied to genomic DNA digested with a restriction endonuclease. The combination of ASO hybridization with PCR made this technique faster, simpler, and much more sensitive. Even a minute quantity of DNA, isolated from a dried blood spot collected on filter paper, can be analyzed by this approach. Both ASO hybridization and restriction enzyme digestion of amplified DNA are easy, rapid, and accurate methods for the localization and confirmation of mutations that are determined through protein analyses or are predicted on the basis of electrophoretic and chromatographic analyses.

The development of PCR has led to a new approach for sequencing human globin genes, bypassing the need to clone the gene of interest. The PCR produces enough DNA that can be used directly for dideoxy sequencing by the method of Sanger et al (58) and direct sequence analysis of amplified DNA has been applied to identify structurally different Hb variants.

Application of Micromethodology to the Identification of $10 \mathrm{Hb}$ Variants. The studies reported in this dissertation clearly demonstrate the advantages of the HPLC and DNA methodologies in the characterization of $\mathrm{Hb}$ variants. The identification of $10 \mathrm{Hb}$ variants (two $\alpha$, four $\beta$, three $\gamma$, and one extended $B$ chain variants) has been presented; seven of these are 
new, while three were previously described in only a few patients. Six were clinically silent, while four were unstable $\mathrm{Hb}$ variants (Sun Prairie, Yokohama, Volga, and Montreal) resulting in a hemolytic anemia in the carriers. Many were detected and characterized by different HPLC procedures. However, for some $\mathrm{Hb}$ variants, a simultaneous identification at the protein and DNA levels is given to show the advantage and necessity of the DNA methodology for their complete characterization.

All the Hb variants described were initially analyzed by an electrophoretic procedure (IEF or starch gel electrophoresis). Five Hb variants, namely Hbs Sun Prairie, Zengcheng, Yokohama, Volga, and F-Jiangsu, did not show an altered electrophoretic mobility and could not be separated from Hbs A or F on IEF or starch gel electrophoresis. Comparable results were obtained with the cation exchange HPLC procedure; Hb variants with an altered electrophoretic mobility were also detectable by this method. The only exception was Hb Iowa, that was detected by IEF as a band very close to $\mathrm{Hb}$ A but could not be separated on cation exchange HPLC.

All abnormal globin chains were detected and quantitated on reversed phase HPLC, even when other procedures failed to indicate the presence of an abnormal $\mathrm{Hb}$. However, al though all of the variants presented here were detectable by this method, other known Hb variants cannot be detected because of the equal polarities of the substituted and introduced amino acid residues. An example is the abnormal $\mathrm{B}$ chain of the unstable $\mathrm{Hb}$ variant $\mathrm{Hb}$ Köln. Fortunately, a combination of reversed phase HPLC for chain separation and IEF or cation exchange HPLC for the Hb separation, allows the detection of most variants. Currently, these three procedures are the methods of choice for the detection and quantitation of Hb variants.

The precise molecular characterization of a particular $\mathrm{Hb}$ variant is greatly facilitated by HPLC methodology and DNA analyses, the advantages of which are best shown in the studies of the three $\mathrm{Hb}$ variants presented in Chapters $3 b, 5 a$, and 7 . The detection and characterization of the unstable a chain variant $\mathrm{Hb}$ Sun Prairie would have been nearly impossible without HPLC methodology. The presence of an unstable Hb variant would probably not have been considered because the stability tests were essentially negative. The detection of a small zone in the Vydac $C_{4}$ HPL chromatogram was the first definitive indication for the presence of an abnormal chain. Preparative reversed phase HPLC has allowed the isolation of the abnormal chain in pure form and in a sufficient amount for peptide analyses, and identification of the Ala->Pro substitution at position 130 of the a chain by amino acid analyses. The confirmation of the $\mathbf{G}->C$ mutation in CD 130 , and its localization on the a2-globin gene, was obtained by dot-blot analyses of amplified a2- and al-globin genes with the CD 130 Ala and CD 130 Pro probes.

The mutation leading to $\mathrm{Hb}$ Yokohama has been characterized at both the protein and DNA level. Apart from the positive stability test, the only evidence for the presence of an abnormal Hb was the extra zone on the reversed phase HPLC for chain separation. The identification of the Leu->Pro substitution was obtained after isolation of the abnormal $\mathrm{Hb}$ with high concentrations of salts, separation and isolation of the abnormal $\beta$ 
chain by CM-52 column chromatography, followed by separation of the tryptic peptides by reversed phase HPLC and amino acid analyses. This was not only a time-consuming but also a difficult approach, since the isolated $B X$ chain was contaminated with normal $B$ chain. In order to confirm the observed substitution, and to present an easier approach for its characterization, DNA analyses were made. The direct sequence analysis of amplified DNA readily localized the $T->C$ substitution at $C D 31$ of the $\beta$ gene. This was indeed a much easier and faster approach for the identification of this abnormal variant.

Finally, in the case of Hb Montreal, the direct DNA sequence analysis was not only easier, it was also the only approach to be taken for determining the nt changes and predicting the likely mechanism(s) responsible for the occurrence of this most unusual $\mathrm{Hb}$ variant. The protein structural analyses of $\mathrm{Hb}$ Montreal predicted the deletion of three amino acid residues (Asp, Gly, and Leu) at positions 73,74 , and 75 , and an insertion of four different residues (Ala, Arg, Cys, and Gln) at the same location in the $\beta$ chain. These were indeed very unusual changes, and the nt alterations could not be predicted from the amino acid variations. The data from the DNA sequence analyses showed the sequence changes in the $\beta-g l o b i n$ gene of the abnormal chromosome and allowed the prediction of the molecular mechanism leading to the observed changes. The repetitive AGT6 sequences flanking the observed changes suggested that a mispairing involving the AGTG sequences at CDs 66 and 67 and at CDs 72 and 73 of the normal $\beta$-globin gene caused a duplication of a 16 bp segment. Two short deletions ( 3 and $10 \mathrm{nts}$ ) occurring in the $3^{\prime}$ end of the duplication and further downstream must have resulted in the sequences observed in the $\beta$-Montreal gene.

The above data demonstrate that HPLC is indeed a sensitive and powerful technique, and is the method of choice for structural analyses of abnormal Hbs in protein chemistry laboratories. However, the introduction of direct sequence analysis of amplified DNA has provided a new and promising approach to the identification of abnormal Hbs. This approach has many advantages over protein analyses; it is faster and more sensitive, it does not require costly equipment, and allows the direct detection of the gene defect. It appears that the identification of Hb variants, especially unstable variants, that are often present in minute amounts, will increasingly depend on this approach.

Unstable $\mathrm{Hb}$ variants represent a major group of clinically significant Hb variants. The term "unstable Hb variant" refers to the group of variants that have an instability sufficient to produce intracellular Hb precipitation, resulting in a hemolytic anemia in the carriers. The disease varies according to the $\mathrm{Hb}$ variant present and it may produce a mild, moderate, or severe hemolytic anemia. However, acute episodes of severe hemolytic anemia may be seen in all cases, secondary to bacterial or viral infections, and exposure to chemical oxidants. The most important factor in the treatment of the hemolytic anemias, secondary to the presence of unstable Hbs, is an early diagnosis. Once the diagnosis is reached, supportive and preventive measures can be instituted, such as administration of folic acid, prevention and prompt treatment of infections, and avoidance of oxidant drugs. Splenectomy can be beneficial, and partial correction 
of the anemia has been achieved in some instances. An early diagnosis of the unstable $\mathrm{Hb}$ variant can prevent unnecessary diagnostic procedures and inappropriate therapeutic interventions.

\section{CONCLUSIONS}

Methodology developed for detection and identification of $\mathrm{Hb}$ variants has been greatly modified over the years. IEF for initial detection of abnormal Hbs, cation exchange HPLC for separation and quantitation of abnormal Hbs, and reversed phase HPLC for separation and quantitation of abnormal globin chains, are currently the methods of choice. Although reversed phase HPLC for peptide analyses is the most useful technique for structural characterization of abnormal Hbs at the protein level, the identification of Hbs with complex structures and with distinct physicochemical properties, will increasingly depend on the application of DNA methodology, i.e. direct sequence analysis of amplified DNA.

Recent developments in the studies of $\mathrm{Hb}$ and its variants have led to revolutionary insights into modern molecular genetics; the contribution of the $\mathrm{Hb}$ genes and hemoglobinopathies to our understanding of molecular genetics continues. However, this has not been a one-way benefit, because the increasing technology of molecular genetics has improved our understanding of the clinical disorders comprising the hemoglobinopathies, and has greatly facilitated their diagnosis. There is no better example than this blending of science and clinical medicine to the mutual benefit of both fields.

\section{REFERENCES}

1. Pauling, L., Itano, H., Singer, S.J., and Wells, I.C.: Sickle cell anemia, a molecular disease. Science, 110:543, 1949.

2. International Hemoglobin Information Center. Hemoglobin, 17:89, 1993.

3. Huisman, T.H.J.: The structure and function of normal and abnormal hemoglobins. In Bailierre's Clinical Haematology, edited by D.J. Weatherall and D.R. Higgs, Vol. 6, page 1, W.B. Saunders Company, London, 1993.

4. Itano, H.A. and Neel, J.V.: A new inherited abnormality of human hemoglobin. Proc. Natl. Acad. Sci. USA, 36:613, 1950.

5. Itano, H.A.: A third abnormal hemoglobin associated with hereditary hemolytic anemia. Proc. Nat1. Acad. Sci. USA, 37:775, 1951.

6. Schneider, R.G.: Paper electrophoresis of hemoglobin as a practical method of differentiating various types of sickle cell disease and of Hemoglobin C trait. Texas Rep. Biol. Med., 11:352, 1953.

7. Smith, E.W. and Conley, C.L.: Filter paper electrophoresis of human hemoglobins with special reference to the incidence and clinical significance of Hemoglobin C. Bu11. Johns Hopkins Hosp., 93:94, 1953.

8. Smithies, 0.: Zone electrophoresis in starch gels: Group variations in the serum proteins of normal human adults. Biochem. J., 61:629, 1955. 
9. Robinson, A.R., Robson, M., Harrison, A.P., and Zuelzer, W.W.: A new technique for differentiation of hemoglobins. J. Lab. Clin. Med., $50: 745,1957$.

10. Drysdale, J.W., Righetti, P., and Bunn, F.: The separation of human and animal hemoglobins by isoelectric focusing in polyacrylamide gel. Biochim. Biophys. Acta, 229:42, 1971.

11. Righetti, P.G., Gianazza, E., Bianchi-Bosisio, A., and Cossu, G.: Conventional isoelectric focusing and immobilized $\mathrm{pH}$ gradients for hemoglobin separation and identification. In The Hemoglobinopathies, edited by T.H.J. Huisman, Methods in Hematology, Vol. 15, page 47, Churchill Livingstone, Edinburgh, 1986.

12. Schroeder, W.A. and Huisman, T.H.J.: The Chromatography of Hemogiobin, Clinical and Biochemical Analysis, Vol. 9, Marcel Dekker, Inc., New York, 1980.

13. Huisman, T.H.J. and Prins, H.K.: The chromatographic behavior of different abnormal human hemoglobins on the cation exchanger Amberlite IRC-50. Clin. Chim. Acta, 2:307, 1957.

14. Allen, D.W., Schroeder, W.A., and Balog, J.: Observations on the chromatographic heterogeneity of normal adult and fetal human hemoglobin: A study of the effects of crystallization and chromatography. J. Am. Chem. Soc., 80:1628, 1958.

15. Schenk, A.G. and Schroeder, W.A.: The relation between minor components of whole normal human adult hemoglobins as isolated by chromatography and starch block electrophoresis. J. Am. Chem. Soc., 83: $1427,1961$.

16. Huisman, T.H.J., Martis, E.A., and Dozy, A.: Chromatography of hemoglobin types on carboxymethylcellulose. J. Lab. Clin. Med., 52:312, 1958.

17. Huisman, T.H.J. and Meyering, C.A.: Studies on the heterogeneity of hemoglobin. I. The heterogeneity of different human hemoglobin types in carboxymethylcellulose and in Amberlite IRC-50 chromatography: Qualitative aspects. Clin. Chim. Acta, 5:103, 1960.

18. Meyering, C.A., Israels, L.A.M., Sebens, T., and Huisman, T.H.J.: Studies on the heterogeneity of hemoglobin. II. The heterogeneity of different hemoglobin types in carboxymethylcellulose and in Amberlite IRC-50 chromatography: Quantitative aspects. Clin. Chim. Acta, $5: 208,1960$.

19. Huisman, T.H.J. and Dozy, A.M.: Studies on the heterogeneity of hemoglobin. IX. The use of tris (hydroxylmethyl)-aminomethane-HCl buffers in the anion-exchange chromatography of hemoglobins. J. Chromatogr., 19:160, 1965.

20. Dozy, A.M., Kleihauer, E.F., and Huisman, T.H.J.: Studies on the heterogeneity of hemoglobin. XIII. Chromatography of various human and animal hemoglobin types on DEAE-Sephadex. J. Chromatogr., 32:723, 1968.

21. Dozy, A.M. and Huisman, T.H.J.: Studies on the heterogeneity of hemoglobin. XIV. Chromatography of normal and abnormal human hemoglobin types on CM-Sephadex. J. Chromatogr., 40:62, 1969.

22. Abraham, E.C., Reese, A., Stallings, M., and Huisman, T.H.J.: Separation of human hemoglobins by DEAE-cellulose chromatography using glycine-KCN-NaCl developers. Hemoglobin, 1:27, 1976-1977. 
23. Huisman, T.H.J. and Dozy, A.M.: Studies on the heterogeneity of hemoglobin. IV. Chromatographic behavior of different human hemoglobins on anion-exchange chromatography (DEAE-cellulose). J. Chromatogr., $7: 180,1962$.

24. Schroeder, W.A., Jakway, J., and Powars, D.: Detection of Hemoglobins $S$ and $C$ at birth: A rapid screening procedure by column chromatography. J. Lab. Clin. Med., 82:303, 1973.

25. Efremov, G.D., Huisman, T.H.J., Bowman, K., Wrightstone, R.N., and Schroeder, W.A.: Microchromatography of hemoglobins. II. A rapid method for the determination of Hemoglobin $A_{2}$. J. Lab. Clin. Med., 83:657, 1974.

26. Huisman, T.H.J., Schroeder, W.A., Brodie, A.N., Mayson, S.M., and Jakway, J.: Microchromatography of hemoglobins. III. A simplified procedure for the determination of Hemoglobin $A_{2}$. J. Lab. Clin. Med., $86: 700,1975$.

27. Efremov, G.D.: Quantitation of hemoglobins by microchromatography. In The Hemoglobinopathies, edited by T.H.J. Huisman, Methods in Hematology, Vol. 15, page 72, Churchill Livingstone, Edinburgh, 1986.

28. Shibata, S.: Separation of hemoglobin chains. Hemoglobin, 4:561, 1980.

29. Clegg, J.B., Naughton, M.A., and Weatherall, D.J.: Abnormal human hemoglobins. Separation and characterization of the $a$ and $B$ chains by chromatography, and the determination of two new variants, Hb-Chesapeake and Hb-J-Bangkok. J. Mol. Biol., 19:91, 1966.

30. Ingram, V.M.: A specific chemical difference between the globins of normal human and sickle-cell anaemia haemoglobin. Nature, 178:792, 1956.

31. Jones, R.T.: Structural studies of aminoethylated hemoglobins by automatic peptide chromatography. Cold Spring Harbor Symposium, Quant. Biol., 29:297, 1964.

32. Schroeder, W.A.: Separation of peptides by chromatography on columns of Dowex 50 with volatile developers. Meth. Enzymol., 15:203, 1972.

33. Schroeder, W.A.: Separation of peptides by chromatography on columns of Dowex 1 with volatile developers. Meth. Enzymol., 15:214, 1972.

34. Gooding, K.M., Lu, K.C., and Regnier, F.E.: High-performance liquid chromatography of hemoglobins. I. Determination of Hemoglobin $A_{2}$. J. Chromatogr., 164:506, 1979.

35. Hanash, S.M. and Shapiro, D.M.: Separation of human hemoglobins by ion exchange high performance liquid chromatography. Hemoglobin, $5: 165,1981$.

36. Gardiner, M.B., Carver, J., Abraham, B.L., Wilson, J.B., and Huisman, T.H.J.: Further studies on the quantitation of the Hemoglobins $A$, $S, C$, and $F$ in newborn babies with different hemoglobinopathies using high pressure liquid chromatography. Hemoglobin, 6:1, 1982.

37. Huisman, T.H.J., Henson, J.B., and Wilson, J.B.: A new high performance liquid chromatographic procedure to quantitate Hemoglobin $A_{1 c}$ and other minor hemoglobins in blood of normal, diabetic, and alcoholic individuals. J. Lab. Clin. Med., 102:163, 1983.

38. Wilson, J.B., Headlee, M.E., and Huisman, T.H.J.: A new high performance liquid chromatographic procedure for the separation and quantitation of various hemoglobin variants in adults and newborn babies. J. Lab. Clin. Med., 102:174, 1983. 
39. Bisse, E. and Wieland, H.: High performance liquid chromatographic separation of human haemoglobins - Simultaneous quantitation of foetal and glycated haemoglobins. J. Chromatogr., 434:95, 1988.

40. Kutlar, A., Kutlar, F., Gu, L-G., Mayson, S.M., and Huisman, T.H.J.: Fetal hemoglobin in normal adults and $\beta$-thalassemia heterozygotes. Hum. Genet., 85:106, 1990.

41. Huisman, T.H.J.: Separation of hemoglobins and hemoglobin chains by high-performance liquid chromatography. J. Chromatogr., 418:277, 1987.

42. Shelton, J.B., Shelton, J.R., and Schroeder, W.A.: Preliminary experiments in the separation of globin chains by high performance liquid chromatography. Hemoglobin, 3:353, 1979.

43. Congote, L.F., Bennett, H.P.J., and Solomon, S.: Rapid separation of the $\alpha, B, G_{\gamma}$, and $A_{\gamma}$ human globin chains by reversed-phase high pressure liquid chromatography. Biochem. Biophys. Res. Commun., 89:851, 1979.

44. Huisman, T.H.J. and Wilson, J.B.: Recent advances in the quantitation of human fetal hemoglobins with different gamma chains. Am. J. Hematol., 9:225, 1980 .

45. Shelton, J.B., Shelton, J.R., and Schroeder, W.A.: High performance liquid chromatographic separation of globin chains on a large-pore $\mathrm{C}_{4}$ column. J. Liq. Chromatogr., 7:1969, 1984.

46. Wilson, J.B., Lam, H., Pravatmuang, P., and Huisman, T.H.J.: Separation of tryptic peptides of normal and abnormal $\alpha, \beta, \gamma$, and $\delta$ hemoglobin chains by high-performance liquid chromatography. J. Chromatogr., 179:271, 1979 .

47. Schroeder, W.A.: High performance liquid chromatography used in structural analyses of hemoglobin variants. In The Hemoglobinopathies, edited by T.H.J. Huisman, Methods in Hematology, Vol. 15, page 142, Churchill Livingstone, Edinburgh, 1986.

48. Southern, E.M.: Detection of specific sequences among DNA fragments separated by gel electrophoresis. J. Mol. Biol., 98:503, 1975.

49. Flavell, R.A., Kooter, J.W., de Boer, E., Little, P.F.R., and Williamson, R.: Analysis of the $\beta-\delta-g l o b i n$ gene in normal and $\mathrm{Hb}$ Lepore DNA: Direct determination of gene linkage and intergene distance. Ce11, 15:25, 1978.

50. Ojwang, P.J., Nakatsuji, T., Gardiner, M.B., Reese, A.L., Gilman, J.G., and Huisman, T.H.J.: Gene deletion as the molecular basis for the Kenya- $G_{\gamma}$-HPFH condition. Hemoglobin, 7:115, 1983.

51. Mavilio, F., Giampaolo, A., Care, A., Sposi, N.M., and Marinucci, M.: The $\delta \beta$ crossover region in Lepore Boston hemoglobinopathy is restricted to a 59 base pairs region around the $5^{\prime}$ splice junction of the large globin gene intervening sequence. Blood, 62:230, 1983.

52. Chang, J.C. and Kan, Y.W.: A sensitive new prenatal test for sickle cell anemia. N. Engl. J. Med., 307:30, 1982.

53. Little, P.F.R., Whitelaw, E., Annison, G., Williamson, R., Kooter, J.M., Flavel1, R.A., Goossens, M., Serjeant, G.R., and Montgomery, D.: The detection of hemoglobin mutants in the direct analysis of human globin genes. Blood, 55:1060, 1980.

54. Nakatsuji, T., Burnley, M.S., and Huisman, T.H.J.: Fetal hemoglobin variants identified in adults through restriction endonuclease gene mapping methodology. Blood, 66:803, 1985. 
55. Saiki, R.K., Scharf, S., Faloona, F., Mullis, K.B., Horn, G.T., Erlich, H.A., and Arnheim, N.: Enzymatic amplification of $B-g l o b i n$ genomic sequences and restriction site analysis for diagnosis of sickle cell anemia. Science, 230:1350, 1985.

56. Saiki, R.K., Chang, C.A., Leveson, C.H., Warren, T.C., Boehm, C.D., Kazazian, H.H., Jr., and Erlich, H.A.: Diagnosis of sickle cell anemia and $\beta$-thalassemia with enzymatically amplified DNA and nonradioactive allele specific oligonucleotide probes. N. Engl. J. Med., 319:537, 1988.

57. Saiki, R.K., Bugawan, T.L., Horn, G.T., Mullis, K.B., and Erlich, H.A.: Analysis of enzymatically amplified $\beta-g l o b i n$ and HLA-DQ $\alpha$ DNA with allele-specific oligonucleotide probes. Nature, 324:163, 1986.

58. Sanger, F., Nicklen, S., and Coulson, A.R.: DNA sequencing with chain-terminating inhibitors. Proc. Natl. Acad. Sci. USA, 74:5463, 1977. 


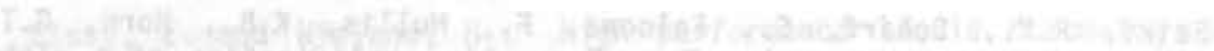

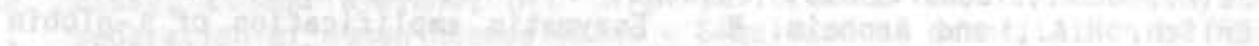

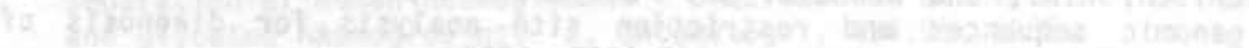
(49.

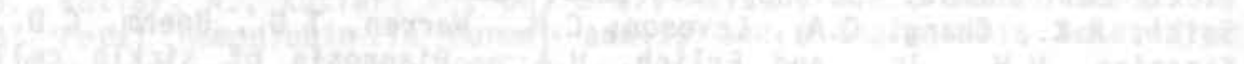

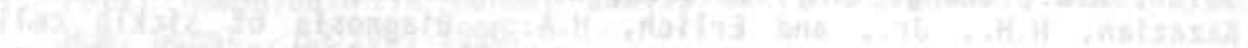

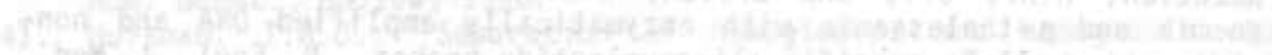

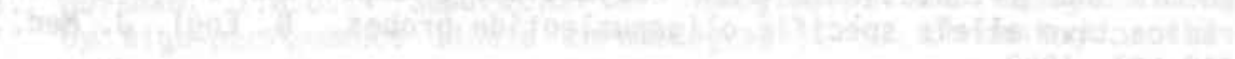

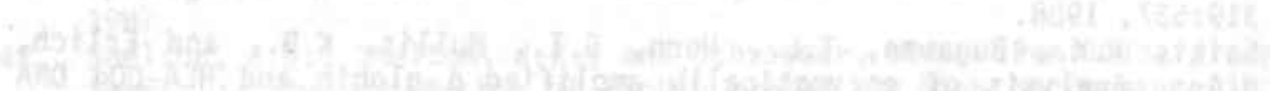

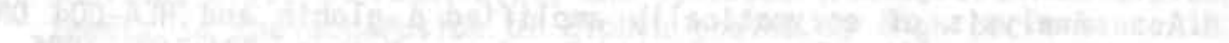

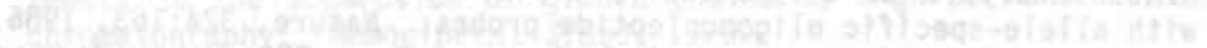

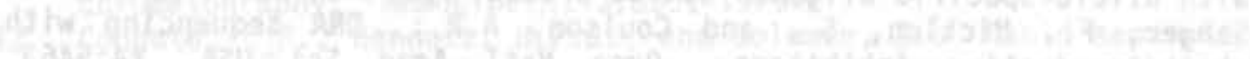

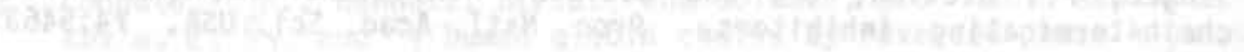

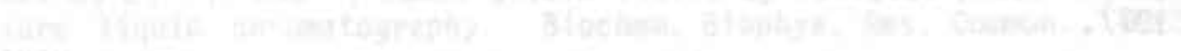
14:20.

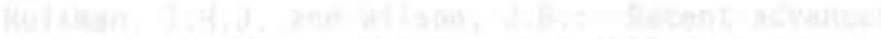

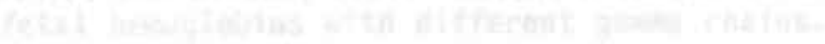

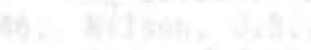
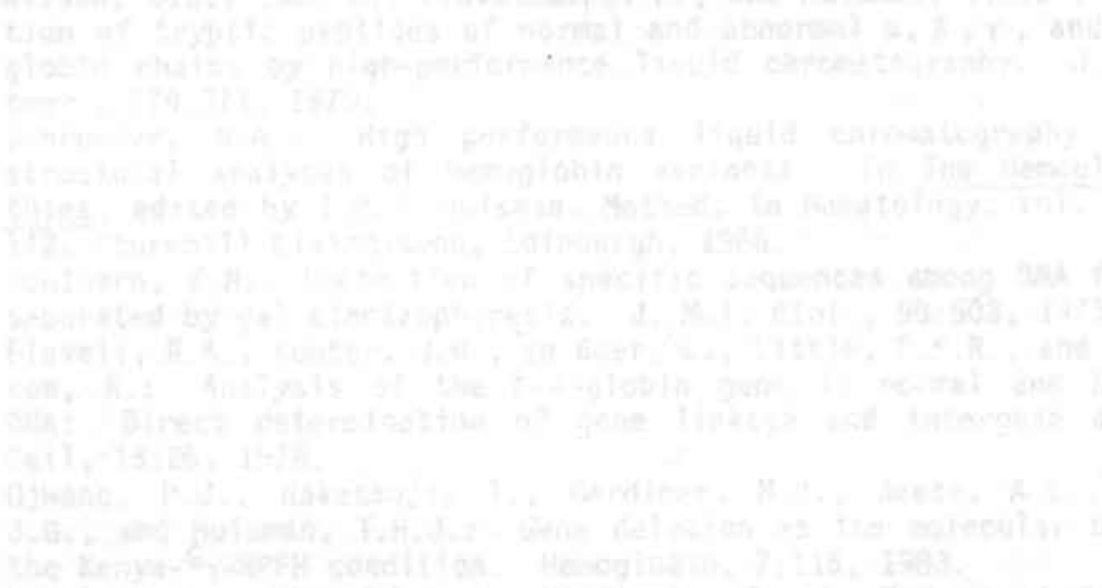

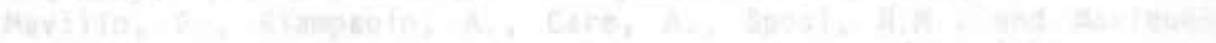

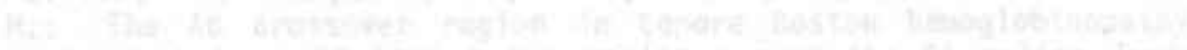

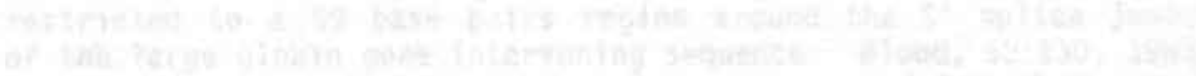

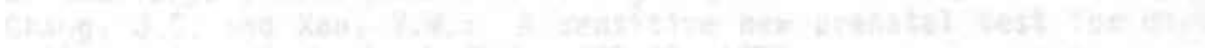

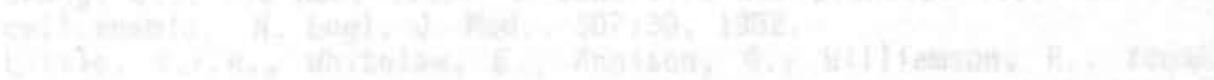

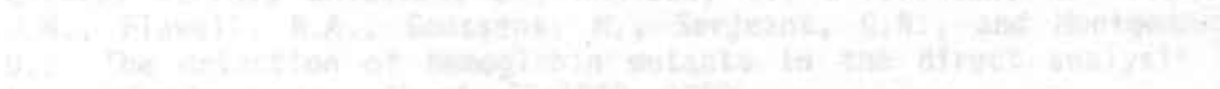

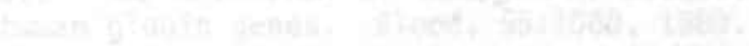

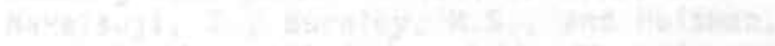

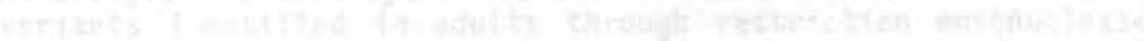

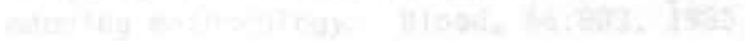




\section{CHAPTER 9}

S U M A A R Y 


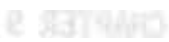

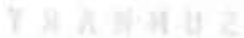




\section{CHAPTER 9}

\section{SURMARY}

A major aim of the studies reported in this dissertation is to demonstrate the usefulness of the HPLC techniques and DNA methodology for the characterization of rare $\mathrm{Hb}$ variants, and to contribute to our understanding of molecular mechanisms that are responsible for the occurrence of some unusual $\mathrm{Hb}$ abnormalities.

A review of the literature is presented in Chapter 1 and includes a description of structure and structure-function relationship of the $\mathrm{Hb}$ molecule, organization and fine structure of the globin genes, and their developmental expression. General aspects of the normal human $H b$, including those predominantly present in the embryo, the fetus, and newborn baby, as well as that in the normal adult, are presented. This chapter also includes a summary of the molecular defects responsible for the occurrence of known Hb variants. Most abnormal Hbs have occurred as a result of mutations in the coding regions of the globin genes that change the sequence. Thus, most of the abnormal Hbs are characterized by single amino acid substitutions, but some have two amino acid substitutions within the same chain, or have longer or shorter chains. Others are fusion or hybrid Hbs that have arisen through crossovers between misaligned chromosomes. Clinically significant variants are reviewed separately; they are classified in four groups: The Hb S syndromes, the unstable Hbs resulting in hemolytic anemia in the carriers, the Hbs with high $\mathrm{O}_{2}$ affinity leading to erythrocytosis, and the $\mathrm{Hbs}$ with $\mathrm{low}_{2}$ affinity and $\mathrm{Hb} \mathrm{M}$ variants, both leading to congenital cyanosis. An updated alphabetical list of known Hb variants is provided as an addendum to this chapter.

The methodologies used for the identification of the Hb variants described in this dissertation are presented in Chapter 2.

Chapter 3 concerns the identification of two $\alpha$ chain variants by HPLC. Chapter 3a describes the characterization of a new $\mathrm{Hb}$ variant with an Asp-> His substitution at position $\alpha 78$. The variant, Hb Davenport, was detected in two members of a Caucasian family. Mild acidic hydrolysis of the large aT-9 peptide, followed by the separation of the resulting fragments by reversed phase HPLC, and amino acid sequence analysis of a few of these fragments, greatly facilitated the identification of this substitution. Asn $a 78$ is not involved in any major contact with the heme group or between globin subunits, and its replacement by another amino acid is not likely to affect $\mathrm{Hb}$ stability; carriers of $\mathrm{Hb}$ Davenport are therefore not expected to be clinically affected.

The second observation of the Hb Sun Prairie [ $\alpha_{2} 130$ (H13)Ala->ProB 2 ] anomaly is described in Chapter 3b. The variant was detected in a 20-yearold Indian male with clinical and hematological features of chronic hemolytic anemia. The identification of this variant would have been virtually impossible without HPLC; the only definitive indication for the presence of an abnormal $\mathrm{Hb}$ was the small extra peak on the reversed phase HPL chromatogram. The preparative reversed phase HPLC permitted the isolation of the 
abnomal chain in pure form. Dot-blot hybridization analysis of the amplified a2-and al-globin genes, using CD $130 \mathrm{Ala}$ and CD 130 Pro oligonucleotide probes, localized the G->C substitution in the $\alpha 2-g l o b i n$ gene. The study provided the interesting observation that the $\alpha$-Sun Prairie chain has a greater affinity for the $\delta$ chain than for the $\beta$ chain.

The characterization of two new $\beta$ chain variants by HPLC is presented in Chapter 4. Chapter 4a deals with the identification of Hb Iowa, which was observed in a Black infant and her mother. The baby was also heterozygous for $\mathrm{Hb} S$. Structural characterization through amino acid analysis of tryptic peptides of the $A E-\beta X$ chain showed a Giy->Ala substitution at position $B 119$ (GH2). The replacement of the B119 Gly does not affect the function of the $\mathrm{Hb}$ molecule, despite its involvement in molecular contacts between the $\alpha 1$ and $\beta 1$ chains.

The use of IEF, cation exchange HPLC, and reversed phase HPLC for screening $\mathrm{Hb}$ abnormalities has led to the detection of a new $B$ chain variant, namely $\mathrm{Hb}$ Zengcheng. The identification of this variant is described in Chapter 4b. Hb Zengcheng [ $\alpha_{2}{ }_{2}{ }_{2} 114(\mathrm{Gl})$ Leu->Met] was observed in a Chinese newborn. The leucine residue at position $B 114$ is internally located and is not involved in any major contact with the heme or globin chains; the Leu->Met replacement is not expected to alter the physicochemical or functional properties of the variant.

Chapter 5 concerns the detection of two unstable $\beta$ chain variants. Their identification was made by both protein and DNA analyses. Hb Yokohama or $a_{23} 2_{21}(B 13)$ Leu->Pro, was observed in a young boy from the former Yugoslavia, who presented with a severe transfusion-dependent hemolytic anemia. The detection of this Hb variant by reversed phase HPLC is presented in Chapter 5a. Its characterization was by HPLC analyses on material obtained by high salt precipitation and by direct sequencing of amplified DNA. The detection of $\mathrm{Hb}$ Volga or $\alpha_{2} B_{2} 27$ (B9)Ala->Asp in a 5-year-old boy from Bosnia and Hercegovina, who suffered from severe hemolytic anemia, is presented in Chapter 5b. The variant was observed by reversed phase HPLC and the structural characterization was by HPLC analysis. An easier approach for the detection of the Hb Volga mutation by Ava II digestion of amplified DNA is also described. The patients with Hb Yokohama and $\mathrm{Hb}$ Volga were born to healthy parents, without a Hb abnormality. Paternity studies included an evaluation of the polymorphisms of the HLA DQa locus and provided data that confirmed a de novo mutation in both patients.

Chapter 6 describes the detection of three new $\gamma$ chain variants, and their characterization by HPLC. Preparative reversed phase HPLC allowed the isolation of the abnormal $\gamma$ chains in pure form, thus greatly facilitating their characterization. During a survey of cord blood samples from heal thy Chinese newborns, a new $A_{\gamma}$ variant was detected. The structural characterization of this abnormal variant, named $\mathrm{Hb} F-J j a n g s u$, is presented in Chapter 6a. Structural analyses of the isolated $A_{\gamma} X$ chain demonstrated a $\sqrt{a}$ ]->Met substitution at position $A_{\gamma} 134$, thus creating a rather unique Met-Met sequence. The location of the second methionine residue in the $\gamma^{T-15}$ peptide significantly influences the chromatographic properties of the abnormal $A_{\gamma}$ chain. The identification of $H b F$-Catalonia or $\alpha_{2} G_{Y 2}{ }^{15}$ (A12) Trp->Arg is presented in Chapter 6b. The variant was observed in 
two Spanish babies. Different HPLC procedures allowed the identification of the Trp->Arg substitution in only a minute quantity of the $G_{\gamma} X$ chain. Chapter 6c describes the structural characterization of Hb F-Brooklyn. The variant was detected in a 2-month-old Caucasian female during a routine screening of blood samples from newborn babies. The variant was detectable on IEF, cation exchange HPLC, and reversed phase HPLC. Structural analyses identified a Lys->Glu substitution at position $G_{\gamma} 66$. It is not yet known whether the substitutions observed in these three new $\gamma$ chain anomalies have any effect on the functional or physicochemical properties of the variants; such studies were not possible due to lack of material.

Chapter 7 presents the identification of the unstable Hb Montreal with a deletion of three amino acid residues (Asp, Gly, Leu) at positions 73,74 , and 75 of the $\beta$ chain, and an insertion of four different residues (Ala, Arg, Cys, Glu) at the same location. The variant was observed in a 7-year-old boy suffering from a moderate hemolytic anemia. The parents of the boy and two of his siblings were normal. The introduction of an extra amino acid residue, and of the changes in the crevice where the heme pocket is located, is the likely cause of the instability of this abnormal $\mathrm{Hb}$. The above listed changes were detected by analyzing the tryptic peptides of the $B$-Montreal chain by direct sequencing of amplified DNA, and through hybridization of amplified DNA to specific oligonucleotide probes. DNA sequence analysis allowed the prediction of the likely mechanism(s) responsible for the occurrence of this unusual variant. It is suggested that a mispairing involving the repetitive AGTG sequence at CDS 66 and 67 and at CDs 72 and 73 of the normal $B$ gene caused a duplication of a 16 bp segment, while a deletion of 10 nts due to recombination or slippage, followed by a second short ( 3 bp) deletion during DNA repair could have resulted in the $\mathrm{Hb}$ Montreal anomaly.

The extensive progress made in the studies of $\mathrm{Hb}$ variants was made possible primarily because of the development of more sensitive and rapid methods for their detection and characterization. Some of the more important methodological achievements in the studies of Hb variants are briefly reviewed in Chapter 8 . Different electrophoretic and chromatographic techniques have been developed in the past; they have played an important role in the detection and characterization of numerous $\mathrm{Hb}$ variants. Because of their altered electrophoretic properties most of the common Hb variants were detected some 20 to 30 years ago. The $\mathrm{Hb}$ variants that have been described in recent years are rare abnormalities, and often have mutations that are difficult to detect by electrophoretic procedures or have a rather complex structure. Advanced methodologies such as HPLC procedures and DNA analyses are required for their characterization.

Currently, IEF, cation exchange HPLC, and reversed phase HPLC are the methods of choice for the initial detection and quantitation of $H b$ variants. Reversed phase HPLC for peptide analyses is a most useful technique for structural characterization of abnormal Hbs at the protein level, but will be replaced by sequencing of amplified DNA involving the $\alpha-, B-$, $\delta-$, or $\gamma$-globin genes. The identification of Hbs with more complex structures and with distinct physicochemical properties will depend completely on the application of DNA methodology. 


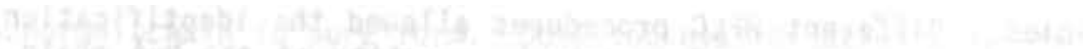

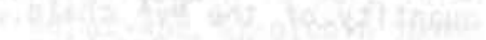

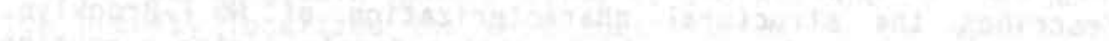

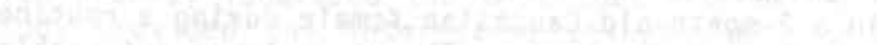

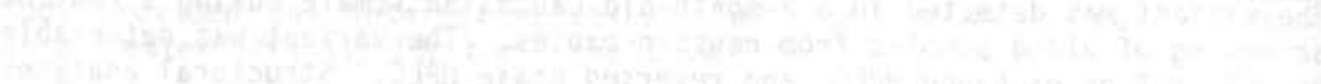

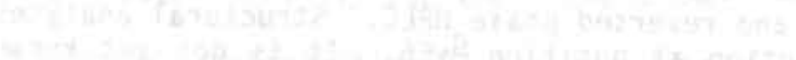

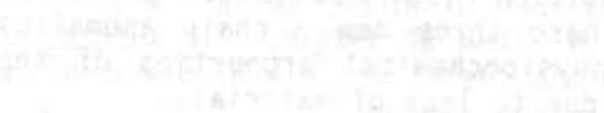




\section{CHAPTER 10}

\section{SAMENVATTING}

Een hoofddoel van de studies, die in dit proefschrift worden beschreven, is de geschiktheid aan te tonen van HPLC-technieken en DNA-methodologie bij het karakteriseren van zeldzame $\mathrm{Hb}$-varianten, en bij te dragen tot een beter begrip van de moleculaire mechanismen, dic verantwoordelijk zijn voor het optreden van enkele ongewone $\mathrm{Hb}$-abnormaliteiten.

In Hoofdstuk 1 wordt een overzicht van de literatuur gegeven en ook wordt de structuur en het structuur-functie verband van het $\mathrm{Hb}$-molecule beschreven. Bovendien wordt de organisatie en de fijn-structuur van de globine-genen beschreven, en hoe de genctische informatic tot uitdrukking wordt gebracht. Algemene aspecten van normaal humaan $\mathrm{Hb}$ worden gepresenteerd, inclusief de belangrijkste aspecten van $\mathrm{Hb}$ afkomstig van de embryo, de foctus, de pasgeboren baby en de volwassene. In dit hoofdstuk wordt bovendien een samenvatting gegeven van de moleculaire defecten welke verantwoordelijk zijn voor het voorkomen van bekende Hb-varianten. De mecste abnormale Hb's zijn het gevolg van een mutatic in de coderende gebicden van de globine genen, waardoor de volgorde is veranderd. Daarom worden de meeste abnormale Hb's gekarakteriseerd door een enkele aminozuur substitutic, maar enkelc hebben twee aminozuur substitutics in dezelfde keten, of hebben een langere of kortere keten. Andere zijn fusie of hybride Hb's dic zijn ontstaan door cross-overs van chromosomen. Klinische belangrijke varianten worden apart besproken. Een indeling in vier klassen is gemaakt: de $\mathrm{Hb}$ S syndromen, de onstabiele $\mathrm{Hb}$ 's, waardoor de dragers hemolytische anemie oplopen, de $\mathrm{Hb}$ 's met hoge affiniteit voor $\mathrm{O}_{2}$ hetgeen leidt tot erythrocytosis, en tenslotte de $\mathrm{Hb}^{\prime}$ 's met lage affiniteit voor $\mathrm{O}_{2}$ en de $\mathrm{Hb} \mathrm{M}$ varianten, dic beide congenitale cyanosis veroorzaken. In een bijlage van dit hoofdstuk wordt een bijgewerkte alfabetische lijst gegeven van bekende $\mathrm{Hb}$-varianten.

De methodologie, die in dit proefschrift wordt gebruikt bij de identificatie van de Hbvarianten, wordt in Hoofdstuk 2 beschreven.

Hoofdstuk 3 behandelt de identificatie van twee $\alpha$-keten varianten door HPLC. In Hoofdstuk 3a wordt de karakterisering van een nieuwe Hb-variant met een Asp $\rightarrow$ His substitutic op positie $\alpha 78$ beschreven. De variant, Hb Davenport, werd aangetoond in twee leden van een blanke familie. De identificatic van deze substitutie werd zeer vergemakkelijkt door gebruik van zwak-zure hydrolyse van de grote $\alpha$ T-9 peptide, gevolgd door scheiding van de gevormde fragmenten door reversed-phase-HPLC en analyse van de aminozuur volgorde van enkele van d'ze fragmenten. Het asparagine residu op de $\alpha 78$ positie is nict betrokken bij cnig belangrijk contact met de haem-groep of tussen globine sub-eenheden, en zijn vervanging door een ander aminozuur beïnvloedt waarschijnlijk niet de $\mathrm{Hb}$-stabiliteit; dragers van $\mathrm{Hb}$-Davenport hebben daarom naar verwachting geen klinische symptomen.

De tweede waarneming van de $\mathrm{Hb}-\mathrm{Sun}$ Prairie $\left[\alpha_{2} 130(\mathrm{H} 13) \mathrm{Ala} \rightarrow \operatorname{Pro} \beta_{2}\right]$ anomalie wordt in Hoofdstuk $3 b$ beschreven. De variant werd aangetoond in een 20 -jarige manlijke Indiaan met klinische en hematologische karakteristieken van chronische hemolytische anemie. De identificatie van deze variant zou praktisch onmogelijk geweest zijn zonder HPLC; de enige definitieve aanwijzing dat het $\mathrm{Hb}$ abnormaal was, was de kleine extra piek op het reversedphase-HPL-chromatogram. Met preparatieve reversed-phase-HPLC was het mogelijk de 
abnormale keten in zuivere vorm te isoleren. Met dot-blot hybridisatie analyse van de vermeerderde $\alpha_{2}$ - en $\alpha_{1}$-genen en gebruik van CD 130 Ala en CD 130 Pro oligonucleotide probes was het mogelijk de $G \rightarrow C$ substitutie in het $\alpha_{2}$-globine gen aan te tonen. De studie leidde tot de interessante waarneming dat de $\alpha$-Sun Prairie keten een grotere affiniteit heeft voor de $S$ keten dan voor de B-keten.

De karakterisering van twee nieuwe B keten varianten door HPLC wordt in Hoofdstuk 4 gepresenteerd. Hoofdstuk $4 \mathrm{a}$ behandelt de identificatie van $\mathrm{Hb}$-lowa, dat werdä aangetroffen in een neger kind en haar moeder. De baby was ook heterozygoot voor $\mathrm{Hb} \mathrm{S}$. Structurele karakterisering door middel van aminozuur analyse van tryptische peptiden van de $A E-\beta{ }_{-k}$ keten toonde aan, dat er een Gly $\rightarrow$ Ala substitutie was opgetreden op positie $B 119(\mathrm{GH} 2)$. De vervanging van $B 119$ Gly heeft geen invloed op de functie van het molecule, ondanks zijn betrokkenheid in moleculaire contacten tussen de $\alpha 1$ - en B1-ketens.

Het gebruik van IEF, cation exchange HPLC en reversed-phase-HPLC bij het zoeken naar $\mathrm{Hb}$ abnormaliteiten leidde tot de ontdekking van een nieuwe B-keten variant, namelijk $\mathrm{Hb}$ Zengcheng. De identificatie van deze variant wordt beschreven in Hoofdstuk $4 \mathrm{~b}$. Hb-Zengcheng $\left[\alpha_{2} B_{2} 114\right.$ (G16)Leu $\rightarrow$ Met] werd waargenomen in een chinese pasgeborene. Het leucine residu op positie B114 ligt binnenin het molecule en is niet betrokken bij enig belangrijk contact met het haem of de globine ketens; daarom wordt niet verwacht dat door de Leu $\rightarrow$ Met uitwisseling de fysicochemische of functionele eigenschappen van de variant zijn veranderd.

In Hoofdstuk 5 wordt de ontdekking van twee onstabiele B-keten varianten beschreven. $\mathrm{Zij}$ werden ontdekt door zowel eiwit- als DNA-analyse. Hb-Yokohama of $\alpha_{2} B_{2} 31$ (B13)Leu $\rightarrow$ Pro werd waargenomen in een jongetje uit het voormalige Joegoslavië, dat leed aan emstige transfusieafhankelijke hemolytische anemie. Hoe deze variant werd aangetoond door reversed-phaseHPLC wordt in Hoofdstuk 5a gepresenteerd. Zijn karakterisering werd uitgevoerd door HPLC analyse van materiaal dat verkregen werd door hoog zout precipitatie en door directe bepaling van de volgorde van vermeerderd DNA. De detectie van $\mathrm{Hb}$-Volga of $\alpha_{2} \mathrm{~B}_{2} 27(\mathrm{~B} 9) \mathrm{Ala} \rightarrow \mathrm{Asp}$ in een 5 -jaar oude jongen uit Bosnië-Hercegovina, die aan ernstige hemolytische anemie leed, wordt in Hoofdstuk $5 \mathrm{~b}$ gepresenteerd. Deze variant werd waargenomen door middel van reversed-phaseHPLC en structureel gekarakteriseerd door HPLC analyse. Een eerdere poging om de $\mathrm{Hb}-\mathrm{V}$ olga mutatie vast te stellen, door Ava II digestie van vermeerderd DNA, is ook beschreven. De patiënten met $\mathrm{Hb}$-Yokohama en $\mathrm{Hb}$-Volga werden geboren uit gezonde ouders zonder $\mathrm{Hb}$ abnormaliteiten. In studies bij de voorouders is een evaluatie van de polymorfismen van de HLA DQa locus betrokken, die bevestigde dat een de novo mutatie in beide ouders was opgetreden.

In Hoofdstuk 6 wordt beschreven hoe drie nieuwe $\gamma$-keten varianten worden aangetoond en hoe zij gekarakteriseerd zijn met HPLC. Door middel van preparatieve reversed-phase-HPLC was het mogelijk de abnormale $\gamma$-keten in zuivere vorm te isoleren, waardoor zijn karakterisering zeer werd vergemakkelijkt. Tijdens een algemene studie van navelstreng bloed monsters van gezonde Chinese pasgeborenen werd een nieuwe A $\gamma$-variant aangetoond. De structurele karakterisering van deze abnormale variant, genaamd $\mathrm{Hb}$-F-jiangsu, wordt in Hoofdstuk 6a gepresenteerd. Met cen structurele analyse van de geïsoleerde AyX-keten werd een Val $\rightarrow$ Met substitutie op positie Ay134 aangetoond, waardoor een tamelijk unieke Met-Met volgorde werd gecreëerd. De lokatie van het tweede methionine residu in het $\gamma \mathrm{T}-15$ peptide beinnvloedt de chromatografische eigenschappen van de A $\gamma$-keten aanzienlijk. De identificatie van $\mathrm{Hb}$-F-Catalonië of $\alpha_{2} \mathrm{G}_{2} 15$ (A12)Trp $\rightarrow$ Arg wordt in Hoofdstuk $6 \mathrm{~b}$ gepresenteerd. De variant werd waargenomen in 
twee Spaanse baby's. Met verschillende HPLC procedures was het mogelijk de Trp $\rightarrow$ Arg substitutie te identificeren in slechts een geringe hoeveclheid van de $\mathrm{G} \gamma \mathrm{X}$-keten. Hoofdstuk $6 \mathrm{c}$ beschrijft de structurele karakterisering van Hb-F-Brooklyn. De variant werd gevonden in een 2maanden-oud blank meisje tijdens een routine onderzoek van bloed monsters van pasgeboren baby's. De variant kon worden aangetoond met IEF, cation exchange HPLC en reversed-phase-HPLC. Door structurele analyse kon een Lys $\rightarrow$ Glu substitutie op positic G $\gamma 66$ worden aangetoond. Het is nog niet bekend of de substitutie, die wordt waargenomen in drie nieuwe $\gamma$ keten anomalieën, enig effect heeft op de functionele of fysicochemische eigenschappen van deze varianten; zulke studies waren niet mogelijk door gebrek aan materiaal.

Hoofdstuk 7 behandelt de identificatie van het onstabiele $\mathrm{Hb}$-Montréal met een deletie van drie aminozuren (Asp, Gly, Leu) op posities 73, 74 en 75 van de B-keten en een insertie van vier verschillende residuen (Ala, Arg, Cys, Glu) op dezelfde lokatie. De variant werd waargenomen in een 7-jaar-oude jongen die leed aan een milde hemolytische anemie. De ouders van de jongen, zijn zuster en zijn broer waren normaal. De introductie van een extra aminozuur residu en de veranderingen in de holte waar de haemzak ligt, zijn de waarschijnlijke oorzaak van de instabiliteit van dit abnormale $\mathrm{Hb}$. De bovengenoemde veranderingen werden gedetecteerd door de tryptische peptiden van de B-Montréal keten te analyseren door de volgorde van vermeerderd DNA direkt te meten of door hybridisatie van dit DNA met specifieke oligonucleotide probes. Analyse van de DNA volgorde maakt het mogelijk te voorspellen wat het (de) waarschijnlijke mechanisme(n) is (zijn) voor het optreden van deze ongewone variant. Het wordt gesuggereerd dat foutieve base paring, waarbij de repeterende AGTG volgorde bij CD's 66 en 67 en bij CD's 72 en 73 van het normale B-gen een rol speelt, een duplicaat van het 16 bp segment veroorzaakt, terwijl een deletie van $10 \mathrm{nts}$ dankzij recombinatie of glijden, gevolgd door een korte $(3 \mathrm{bp}$ ) deletic tijdens DNA reparatie, de abnormaliteit van het $\mathrm{Hb}$-Montréal zou kunnen hebben veroorzaakt.

De aanzienlijke vooruitgang die werd gemaakt bij de studie van $\mathrm{Hb}$-varianten was primair mogelijk door de ontwikkeling van gevoeliger en snellere methoden bij hun detectie en karakterisering. Enkele van de belangrijkste methodologische prestaties in de studie van $\mathrm{Hb}-$ varianten worden kort besproken in Hoofdstuk $\mathbf{8}$. In het verleden zijn verschillende electroforetische en chromatografische technieken ontwikkeld; deze hebben een belangrijke rol gespeeld bij de detectie en karakterisering van talrijke Hb-varianten. Wegens hun veranderde electroforetische eigenschappen zijn de meeste gewone $\mathrm{Hb}$-varianten al reeds 20 tot 30 jaar geleden ontdekt. De $\mathrm{Hb}$-varianten, die de laatste jaren zijn ontdekt, zijn zeldzame abnormaliteiten, die vaak een mutatie hebben die moeilijk op te sporen is met electroforetische procedures, of een tamelijk ingewikkelde structuur hebben. Vergevorderde methodologie zoals HPLC-procedures en DNA-analyses zijn vereist voor hun karakterisering.

Momenteel zijn IEF, cation exchange HPLC en reversed-phase-HPLC de methoden, die als eerste gekozen worden, voor de detectie en de bepaling van het voorkomen van Hb-varianten. Reversed-phase-HPLC voor peptide analyse is een zeer bruikbare techniek voor structurele karakterisering van abnormale Hb's op het eiwit niveau, maar zal worden vervangen door het bepalen van de volgorde van vermeerderd DNA van de $\alpha-\beta-, \delta$ - of $\gamma$-genen. De identificatie van Hb's met complexere structuren en met specifieke fysicochemische cigenschappen zal volledig afhankelijk zijn van de toepassing van DNA-methodologic. 


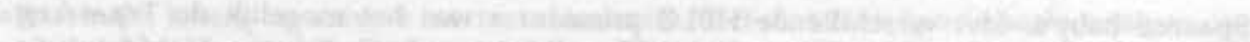
Whe

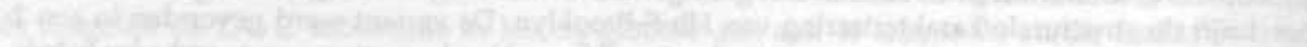

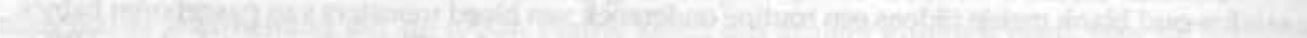

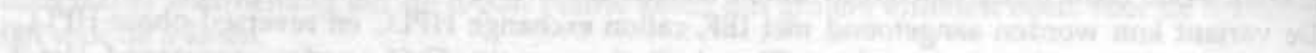

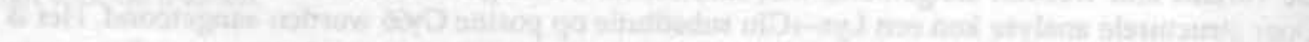

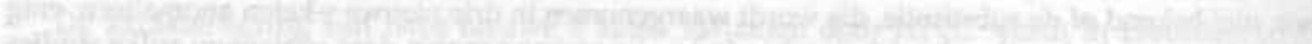

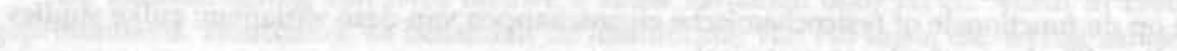

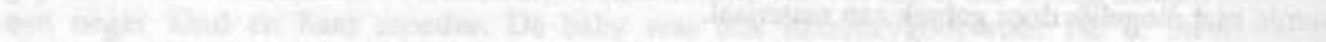

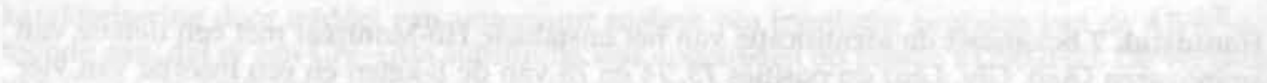

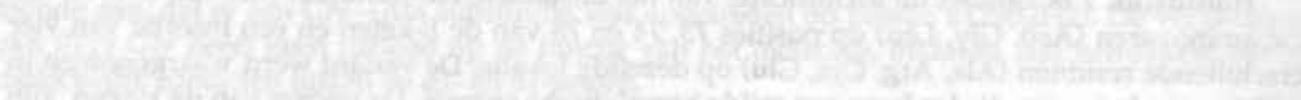

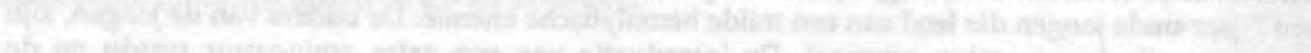

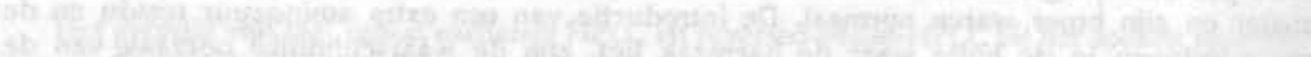

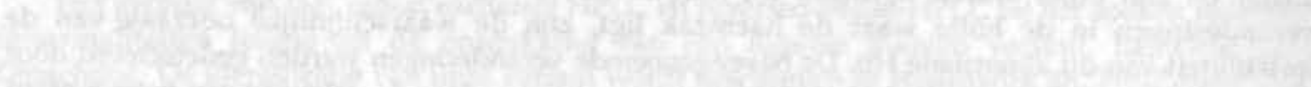

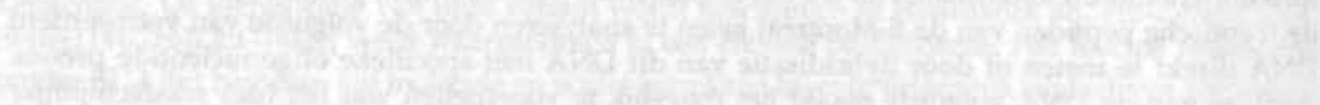

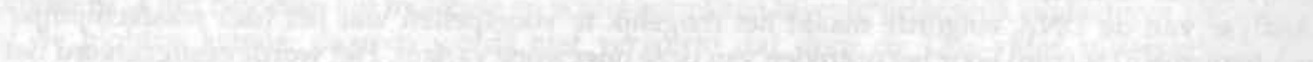

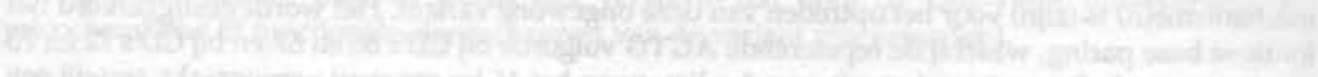

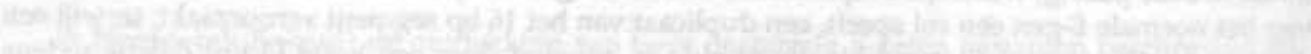

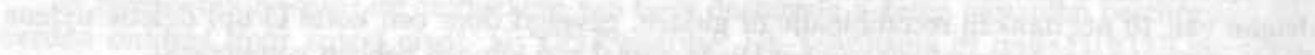

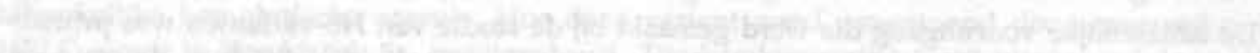

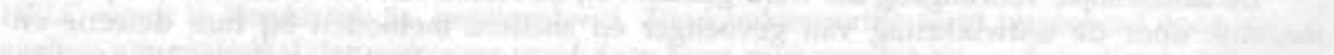

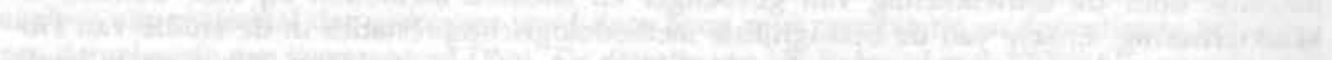

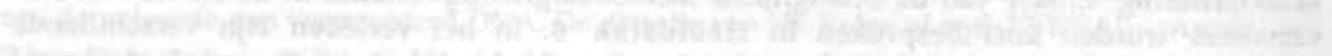

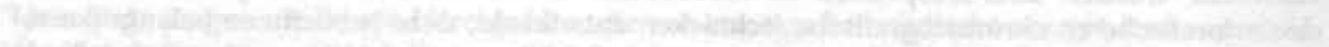

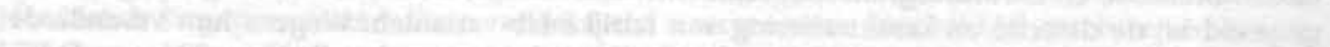

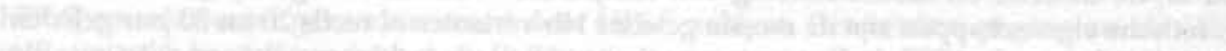

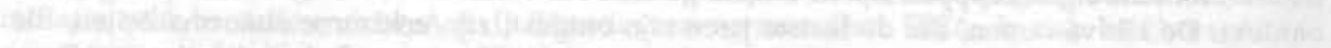

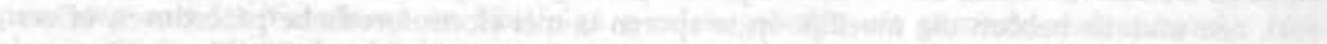

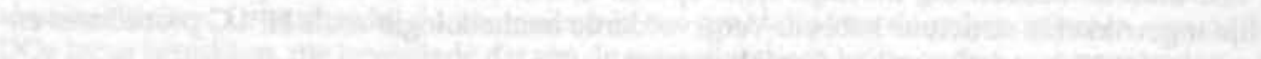

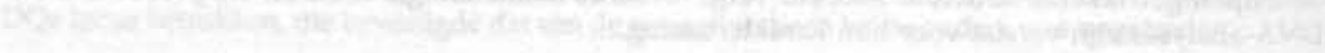

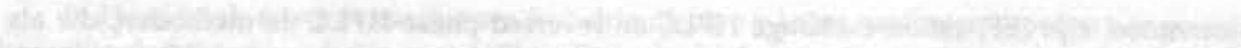

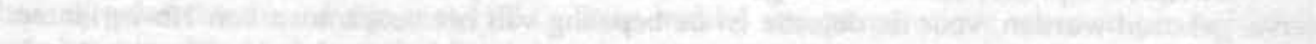

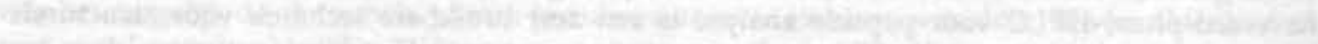

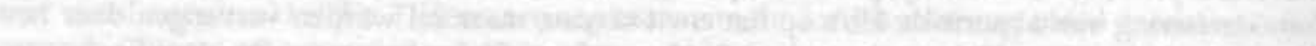

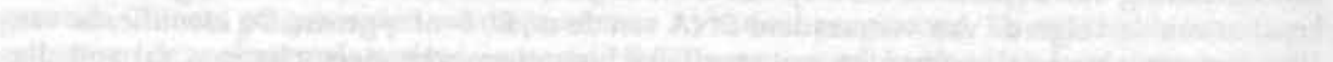

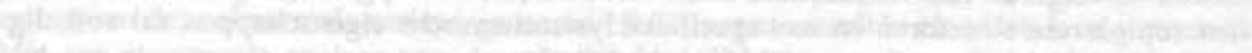

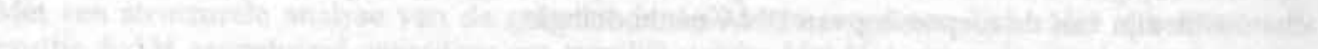

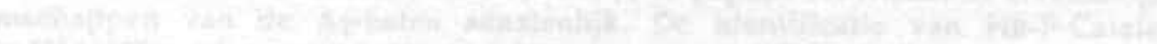

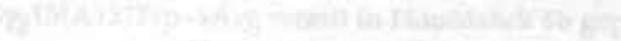




\section{CURRICULUN VITAE}

Name:

Date of Birth:

Sex:

P1ace of Birth:

Citizenship:

Education: University

Research Experience:

Position:

Present Address:
Dijana Plaseska

May 21, 1963

Female

Skopje, Republic of Macedonia

Macedonian

Graduated in 1987 from the Faculty of Medicine at the University of Kiril and Metodoji, Skopje, Republic of Macedonia, with an average grade of $9.9 / 10$.

Postdoctoral Training: 1989-1990 (one year)

Department of Cell and Molecular Biology

Medical College of Georgia, Augusta, GA, USA.

Several years experience in the methodologies of protein analysis used for the study of abnormal hemoglobins. Some DNA analyses that included amplification and sequencing, etc.

Research Assistant

Macedonian Academy of Sciences and Arts

Research Center for New Technologies

Skopje, Republic of Macedonia

Macedonian Academy of Sciences and Arts

Research Center for New Technologies

Bul. "Krste Misirkov" 2, РОВ 428

91000 Skopje, Republic of Macedonia

TEL: +389-91-239-061; FAX: +389-91-115-434 